\title{
Autonomous \& Adaptive Oceanographic Feature Tracking On Board Autonomous Underwater Vehicles
}

\author{
by \\ Stephanie Marie Petillo \\ B.S., Aerospace Engineering, University of Maryland, College Park (2008) \\ Submitted to the Joint Program in Applied Ocean Science \& Engineering \\ in partial fulfillment of the requirements for the degree of \\ Doctor of Philosophy in Oceanographic Engineering \\ at the \\ MASSACHUSETTS INSTITUTE OF TECHNOLOGY \\ and the \\ WOODS HOLE OCEANOGRAPHIC INSTITUTION
}

February 2015

(C)2015 Stephanie M. Petillo.

All rights reserved.

The author hereby grants to MIT and WHOI permission to reproduce and to distribute publicly paper and electronic copies of this thesis document in whole or in part in any medium now known or hereafter created.

Author

Joint Program in Oceanography/Applied Ocean Science \& Engineering Massachusetts Institute of Technology \& Woods Hole Oceanographic Institution November 21, 2014

Certified by

Henrik Schmidt

Professor of Mechanical and Ocean Engineering Massachusetts Institute of Technology Thesis Supervisor

Accepted by

Henrik Schmidt

Chairman, Joint Committee for Applied Ocean Science \& Engineering Massachusetts Institute of Technology

Woods Hole Oceanographic Institution

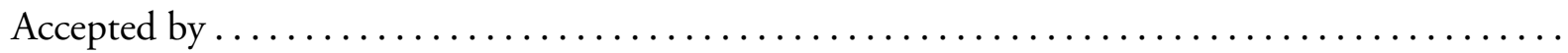

David E. Hardt

Chairman, Committee for Graduate Students Massachusetts Institute of Technology 


\title{
Autonomous \& Adaptive Oceanographic Feature Tracking On Board Autonomous Underwater Vehicles
}

by

Stephanie Marie Petillo

\author{
Submitted to the MIT/WHOI Joint Program in Applied Ocean Science \& Engineering \\ on November 21, 2014, in partial fulfillment of the \\ requirements for the degree of \\ Doctor of Philosophy in Oceanographic Engineering
}

\begin{abstract}
The capabilities of autonomous underwater vehicles (AUVs) and their ability to perform tasks both autonomously and adaptively are rapidly improving, and the desire to quickly and efficiently sample the ocean environment as Earth's climate changes and natural disasters occur has increased significantly in the last decade. As such, this thesis proposes to develop a method for single and multiple AUVs to collaborate autonomously underwater while autonomously adapting their motion to changes in their local environments, allowing them to sample and track various features of interest with greater efficiency and synopticity than previously possible with preplanned AUV or ship-based surveys. This concept is demonstrated to work in field testing on multiple occasions: with a single AUV autonomously and adaptively tracking the depth range of a thermocline or acousticline, and with two AUVs coordinating their motion to collect a data set in which internal waves could be detected. This research is then taken to the next level by exploring the problem of adaptively and autonomously tracking spatiotemporally dynamic underwater fronts and plumes using individual and autonomously collaborating AUVs.
\end{abstract}

Thesis Supervisor: Henrik Schmidt

Title: Professor of Mechanical and Ocean Engineering

Massachusetts Institute of Technology 


\section{Biography}

Stephanie Petillo grew up in Sterling, VA, and Acton, MA, where she attended Acton-Boxborough Regional High School. Her extracurricular activities involved marching band, dancing, Science Olympiad Team, figure skating, Girl Scouts, working on house projects and motor vehicles, riding dirt bikes in her backyard, and learning languages. Stephanie earned her Bachelor of Science Degree in Aerospace Engineering with a minor in Italian Language and Culture from the University of Maryland in College Park, MD, in 2008. While there she was a Team Leader of the Near Space balloon payload project, a Squad Leader of the trombone and baritone horn section of the Mighty Sound of Maryland marching band, an Apartment Leader for the Italian Cluster in the Language House, and she figure skated and raced cars competitively. Stephanie spent her final two summers of college in Woods Hole, MA, as a Guest Student in the WHOI Autonomous Systems Lab working with and deploying underwater gliders in 2006 and as a student at the Sea Education Association conducting oceanographic research and sailing a tall ship across the Pacific Ocean during an SEA Summer Semester in 2007. These experiences influenced her undergraduate research thesis entitled "Evaluation of the Coordinated Sampling Performance of Underwater Gliders in Strong and Variable Currents: A Simulated Case Study in the Chesapeake Bay" and convinced her to switch into oceanographic engineering for graduate school.

Stephanie entered the Massachusetts Institute of Technology/Woods Hole Oceanographic Institution Joint Graduate Program following college to study Oceanographic Engineering. Her Doctoral research, which comprises this thesis, has focused on using autonomous underwater vehicles (AUVs) to perform autonomous and environmentally adaptive sampling of the ocean environment, focusing on underwater feature detection and tracking for more efficient and synoptic data collection with AUVs. Stephanie has enjoyed being on and near the ocean, working with oceanographic vehicles and instrumentation, and seeing her complex underwater vehicle missions work out in the water, and she has had the great opportunity of participating in over a dozen research cruises since she first started working with underwater vehicles in college. Throughout graduate school, Stephanie has continued her many hobbies and activities, adding hiking, farming, sailing, and woodworking to the list.

\section{Acknowledgments}

First of all I would like to thank my advisor, Prof. Henrik Schmidt, for making this thesis possible. He has not only provided me with the opportunity to follow my own path with my $\mathrm{PhD}$ research, but also has been very 
trusting in allowing his grad students to get their hands dirty with AUV deployments on numerous research cruises. My cruise count has certainly topped 10 by now, which is more than I could have hoped for coming into the Joint Program. I can truly say I have had a great time working with Henrik and appreciate all the trips to Italy and other cruise and conference travel he has let me participate in right up until the end of my time here. Thank you!

Second I would like to thank the rest of my thesis committee, Dr. Arjuna Balasuriya, Prof. Pierre Lermusiaux, and Dr. Dana Yoerger, for all of their invaluable input to my research. Thanks to Arjuna for collaborating with me from the beginning of my time at MIT and for providing us with pizza those late nights in the lab doing last-minute SWAMSI payload wiring. Thanks to Pierre for vouching for me during quals and always bending over backwards to get me whatever models or data I needed. Thanks to Dana for all the great stories from AUV deployments and life and for many discussions on plume tracking. I would also like to thank Dr. Hanu Singh for chairing my defense and looking after my progress on the WHOI side of things from the beginning of my time in the Joint Program.

Thank you to Dr. Mike Benjamin for writing and maintaining the IvP Helm, which has made the many virtual and field experiments in this thesis possible. I have also appreciated the many insightful discussion we have had, and I especially appreciate your letting me kidnap more than my fair share of your laptops for a year to run my virtual experiments.

I would also like to thank all the members of the LAMSS lab past and present: Thank you Toby for fixing nearly any software problem we threw at you on cruise and in the office, and for completing our A-team for every cruise we were on together. Thank you also for being my partner in crime on cruise, on travel, and in life. I could not have completed this research nearly as efficiently without you there to devise all sorts of work-arounds when I wanted extra features to make my virtual experiments easier to run. Thank you to Erin and Sheida for being both great friends and lab mates and for teaching me that it is okay to not be the only woman in an engineering lab! Thanks Stephanie for always being excited about my research and hosting craft nights. Thom, thanks for being such a quick learner on cruise and generally a fun person to hang out with. You will be a good replacement for me. Geoff, thanks for always being helpful and taking care of anything we needed for the lab, and thanks for always griping along with me whenever someone was doing something dumb. Tom and Aurthur, thanks for always being positive and being an integral part of the lab. It is good to have the perspective of you Navy guys reminding us that the real world is out there. Nick, thanks for taking over my work with the MSEAS group so it will hopefully not all get shelved once I am done. Alon, it has been fun working with you and listening to all your crazy ideas, even if I miss your media references $75 \%$ 
of the time! Ian and Kevin, thanks for all the fun and serious discussions in the lab when there was a lull in productivity. Kevin, thanks also for always answering my questions about how to jump through the academic and thesis hoops and for treating me like all the other guys in the lab (that is a good thing!). Don, thanks for our discussions that got me started on front tracking and environmental sampling behaviors. Ray and Costas, it was great sharing the lab with you. Hopefully our paths will cross again. It has been a pleasure working with you all.

Thank you also to Pierre, Pat Haley, and Wayne Leslie in the MSEAS group for, again, bending over backwards to get me the models, code, and information I needed for my research.

Thanks to Bluefin Robotics (and especially the operations guys) for the AUV support on every cruise, letting me help with AUV and instrument deployments, and basically training me to service the AUVs so I could do it all myself.

Thanks to all the folks I have worked with at the Centre for Maritime Research and Experimentation (CMRE) in La Spezia, Italy, including David Hughes, Marco Mazzi, Francesco Baralli, Kim McCoy, and the OEX and Autonomy groups who made my thermocline tracking and Internal Wave Detection Experiments possible.

Thanks to Mike Incze, Scott Sideleau, and Don Eickstedt from NUWC Newport for supporting the Champlain '09 experiment for my research.

To the WHOI APO and all those that have taught, supervised, and mentored me since I first came to WHOI as a Guest Student, thank you!

I would like to thank the following programs and sponsors for funding and supporting my research, tuition, and stipend:

- The MIT/WHOI Joint Program

- Government support under and awarded by DoD, Air Force Office of Scientific Research, NDSEG Fellowship, 32 CFR 168a

- The U.S. Office of Naval Research (ONR) GOATS '11 (N00014-11-1-0097) and GOATS '14 (N0001414-1-0214) projects

- The ONR TechSolutions Program Office (Lightweight NSW UUV program) - Technical Support

- The Naval Undersea Warfare Center (NUWC) Division, Newport (Code 25) - Technical Support and Logistics 
Finally, I would like to thank all of my friends from the Joint Program and beyond that have been nothing but supportive throughout my time in grad school. In particular I am grateful to have Derya, Ankita, Kalina, and Heather as my engineering friends who suffered through quals and classes with me in our first few years. I also owe a huge thanks to my family, and in particular my parents, who have always encouraged me to follow my dreams and do what I want to do in life and school. Mom and Dad, you cultivated my love of engineering and science and have bent over backwards to help and be supportive wherever possible throughout school, thesis writing, and all of my extracurricular adventures, and I can't thank you enough! I think this comic sums it up pretty well:
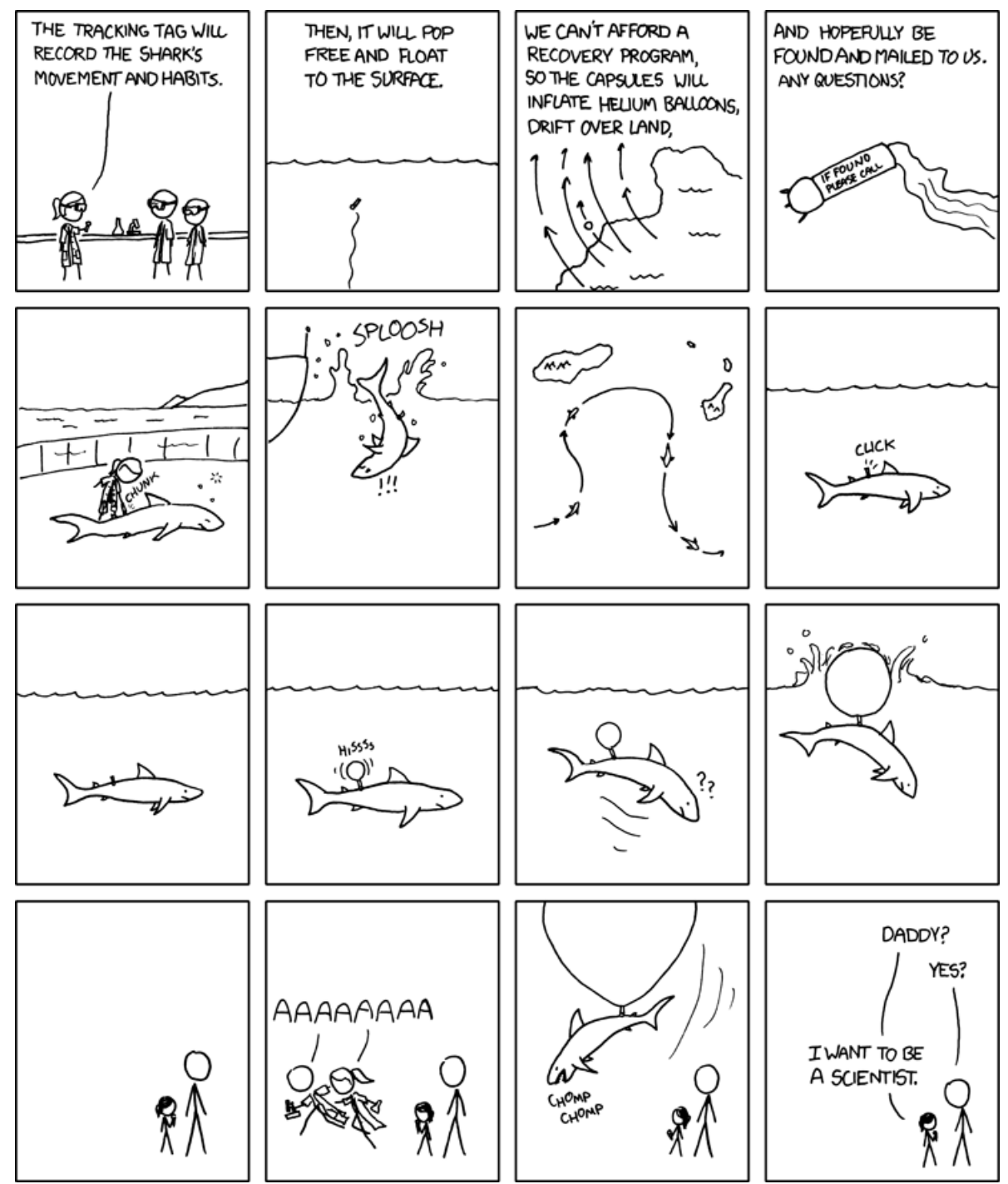

"Completely implausible? Yes. Nevertheless, worth keeping a can of shark repellent next to the bed." http://xkcd.com/585/ 


\section{Contents}

$\begin{array}{llr}1 & \text { Introduction } & 15\end{array}$

1.1 Concepts/Approach . . . . . . . . . . . . . . . . . . . . 16

1.2 Thesis Outline \& Contributions . . . . . . . . . . . . . . . . . . . . 17

2 Background $\quad 21$

2.1 Autonomous Adaptive Sampling . . . . . . . . . . . . . . . . . . 21

2.2 Advantages \& Challenges . . . . . . . . . . . . . . . . . . . 22

2.2 .1 AUVs in the real environment . . . . . . . . . . . . . . . . 22

2.2 .2 AUV Networks . . . . . . . . . . . . . . . . . . . . . 24

2.2.3 Environmentally Adaptive Autonomy \& Autonomous Coordinated Control . . . . 24

2.2 .4 Acoustic Communication . . . . . . . . . . . . . . . . . . . 25

2.2 .5 Data Fusion . . . . . . . . . . . . . . . . . . 26

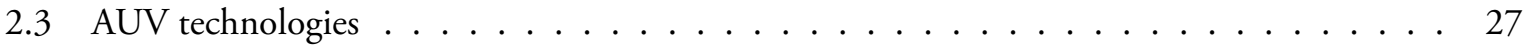

2.3.1 Autonomy Middleware . . . . . . . . . . . . . . . . . . . . . 27

2.3.2 Acoustic Communications . . . . . . . . . . . . . . . . 28

2.3 .3 Sensors \& Instrumentation $\ldots \ldots \ldots \ldots \ldots \ldots \ldots$

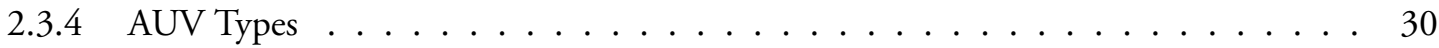

2.4 Spatiotemporal Scales of Ocean Features . . . . . . . . . . . . . . . . 32

2.4.1 Synoptic Sampling vs. Spatiotemporal Aliasing . . . . . . . . . . . . . . 34

2.4.2 AUV Types and Numbers Suited to Different Features' Scales . . . . . . . . . . . . 35

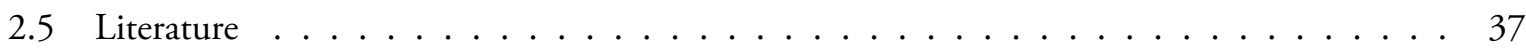

3 Thermocline Tracking: A Proof-of-Concept for Autonomous Adaptive Environmental Assessment and Feature Tracking 39 
3.1 Introduction . . . . . . . . . . . . . . . . . . . . . . . . 39

$3.2 \quad$ Background \& Importance $\ldots \ldots \ldots \ldots \ldots \ldots$

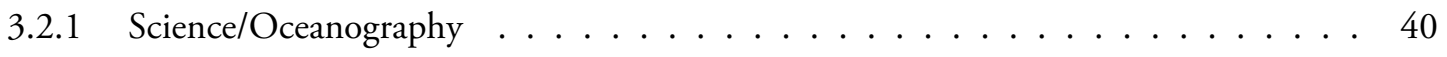

3.2 .2 Technology/Engineering . . . . . . . . . . . . . . . . . 41

3.3 Goals \& Motivation . . . . . . . . . . . . . . . . . . . . . . 41

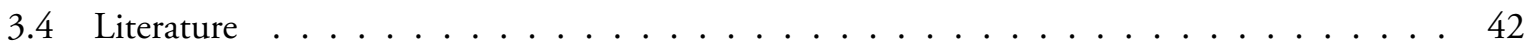

3.5 AAEA \& Feature Tracking: A Novel Approach $\ldots \ldots \ldots \ldots$

3.5 .1 Oceanographic Features . . . . . . . . . . . . . . . . . . . . 43

3.5.2 Defining a Feature Based on Data . . . . . . . . . . . . . . . . 43

3.5 .3 AAEA Process $\ldots \ldots \ldots \ldots \ldots \ldots \ldots \ldots \ldots \ldots \ldots$

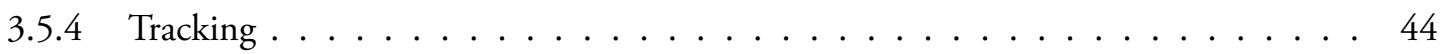

3.6 Tracking the Marine Thermocline $\ldots \ldots \ldots \ldots \ldots \ldots$

3.6 .1 Thermocline Definition . . . . . . . . . . . . . . . . . . . 44

3.6.2 Algorithm: Thermocline Bounds and Maximum . . . . . . . . . . 45

3.6.3 Algorithm Implementation on AUVs: pEnvtGrad . . . . . . . . . . . . . . 47

3.6.4 Virtual Experiments \& Testing . . . . . . . . . . . . . . . . . . . . . 49

3.7 Field Experiments \& Results . . . . . . . . . . . . . . . . . . . . . . . . . . . . . . . . 49

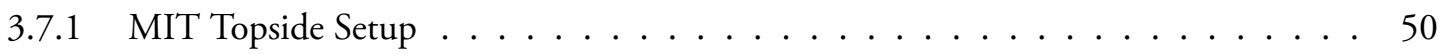

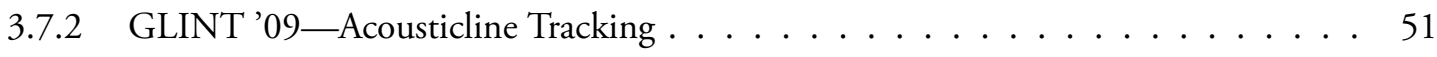

3.7 .3 GLINT' 09 Results . . . . . . . . . . . . . . . . . . . . . . . 52

3.7.4 Champlain '09-Thermocline Tracking . . . . . . . . . . . . . . 54

3.7 .5 Champlain 09 Results . . . . . . . . . . . . . . . . 54

3.7.6 GLINT '10-Thermocline Tracking for Internal Wave Detection . . . . . . . . 56

3.7 .7 GLINT '10 Results . . . . . . . . . . . . . . . . . . . . . 57

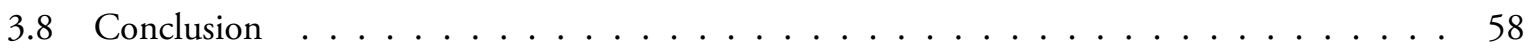

$\begin{array}{lll}4 & \text { Internal Wave Detection Experiment } & \mathbf{6 1}\end{array}$

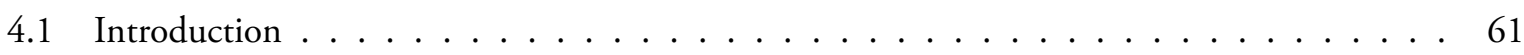

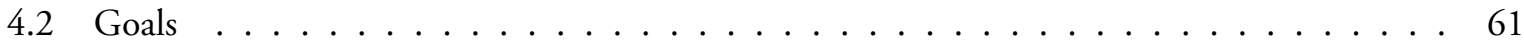

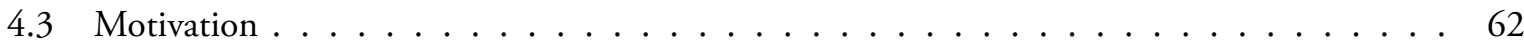

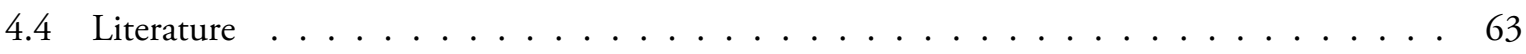

4.5 A Novel Approach to Implementing Internal Wave Detection . . . . . . . . . . . 65 
4.5.1 Hardware Platforms . . . . . . . . . . . . . . . . . 66

4.5 .2 AUV Missions . . . . . . . . . . . . . . . . . . . . . 68

4.5.3 MOOS Processes and IvP Helm Autonomy Behaviors _ . . . . . . . . . . . 69

4.6 Data $\&$ Results . . . . . . . . . . . . . . . . . . . . . 72

4.6.1 Oceanographic Conditions . . . . . . . . . . . . . . . 73

4.6 .2 Mission Execution . . . . . . . . . . . . . . . . . . 73

4.7 Analysis . . . . . . . . . . . . . . . . . . 77

4.8 Receiver Operating Characteristic Curves . . . . . . . . . . . . . . . . 88

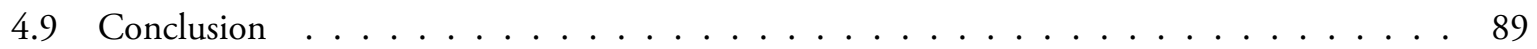

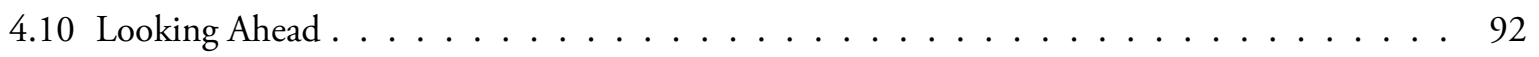

4.10 .1 Further Data Analysis \& Future Experiments . . . . . . . . . . . . . . 92

4.10 .2 Broader Applications . . . . . . . . . . . . . . . . . . 93

5 Front Tracking $\quad 95$

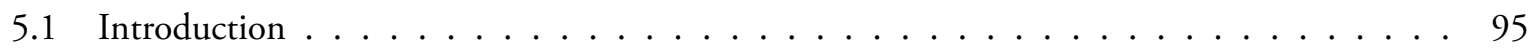

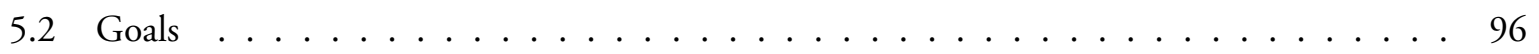

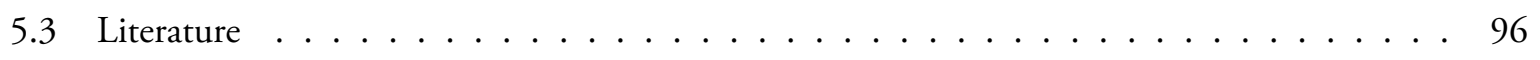

5.4 Novel Concepts \& Approach $\ldots \ldots \ldots \ldots$. . . . . . . . . . . . . 97

$5.52 \mathrm{D} \&$ Multi-AUV Front Tracking . . . . . . . . . . . . . . . . 101

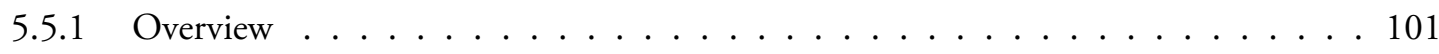

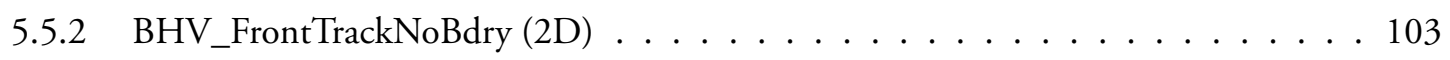

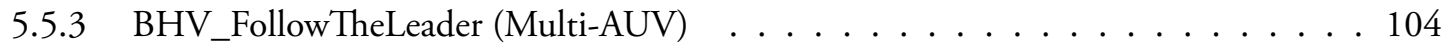

5.6 3D Front Tracking $\ldots \ldots \ldots \ldots \ldots$

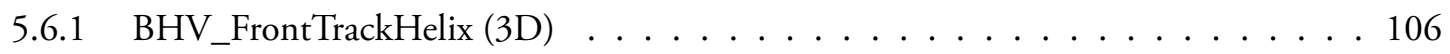

5.7 Virtual Experiments \& Results . . . . . . . . . . . . . . . . . . . . 109

5.7.1 MSEAS 4D Ocean Model Environment . . . . . . . . . . . . . 110

5.7 .2 Preplanned Missions . . . . . . . . . . . . . . . . . . 112

5.7 .3 New Adaptive Missions . . . . . . . . . . . . . . . . . . . 113

5.7.4 Mission Configurations . . . . . . . . . . . . . . . . . . . . . 119

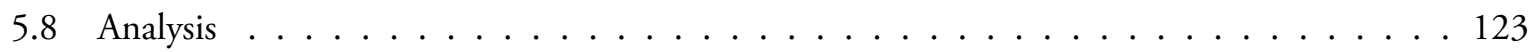

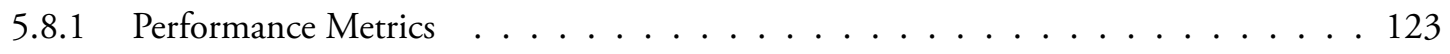

5.8 .2 Data Analysis . . . . . . . . . . . . . . . . 126 
5.9 Receiver Operating Characteristic Curves . . . . . . . . . . . . . . . . . . . . . . 148

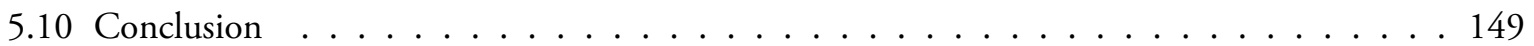

6 Plume Tracking $\quad 155$

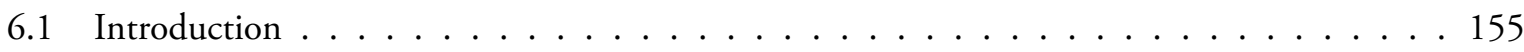

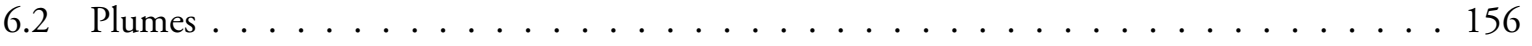

6.2 .1 Hydrothermal Vent Plumes . . . . . . . . . . . . . . . . . . . . 156

6.2 .2 Oil Spills . . . . . . . . . . . . . . . . . . 157

6.2 .3 Harmful Algal Blooms . . . . . . . . . . . . . . . . . . 158

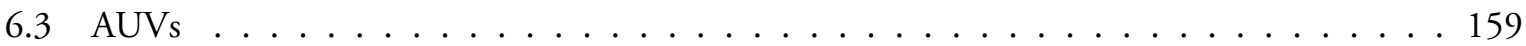

6.4 Plume Tracking Methods . . . . . . . . . . . . . . . . . . 160

6.4 .1 Related Literature . . . . . . . . . . . . . . . . . . . . . 161

6.4 .2 Our Approach . . . . . . . . . . . . . . . . . . . . . . 162

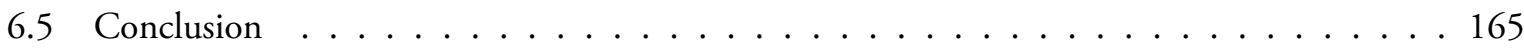

7 Conclusion \& Future Directions $\quad 167$

7.1 Looking Ahead . . . . . . . . . . . . . . . . . . . . . . . . . 169

$\begin{array}{lll}\text { A } & \text { MSEAS Integration } & 171\end{array}$

A.1 LAMSS-MSEAS Interface $\ldots \ldots \ldots \ldots \ldots \ldots \ldots \ldots$. . . . . . . . . . . . . . . . . . . . .

A.1.1 Virtual Experiment Environment . . . . . . . . . . . . . 171

A.1.2 Mission Simulation . . . . . . . . . . . . . . . . . . . . 179

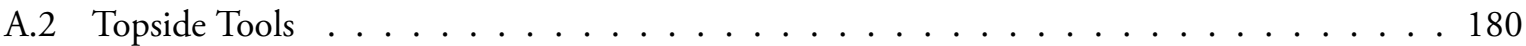

A.2.1 MSEAS Display . . . . . . . . . . . . . . . . . 180

A.2.2 CTD Display . . . . . . . . . . . . . . . . . 180

B Constructing a Distributed AUV Network for Underwater Plume-Tracking Operations 183

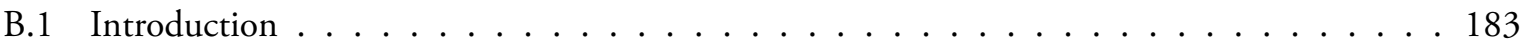

B.2 Spatiotemporal Aliasing Problem . . . . . . . . . . . . . . 185

B.3 Advantages and Challenges of an AUV Network . . . . . . . . . . . . . . 186

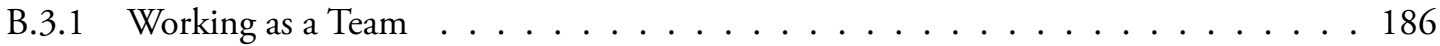

B.3.2 Autonomous Coordinated Control . . . . . . . . . . . . . . . . . . . . . 187

B.3.3 Acoustic Communication . . . . . . . . . . . . . . . . . . . . 187 
B.3.4 Data Fusion . . . . . . . . . . . . . . . . . . 188

B.4 Adaptive Behavior Implementation ～. . . . . . . . . . . . . . . . . . . . . 189

B.4.1 Thermocline Tracking as a Proof-of-Concept . . . . . . . . . . . . . . . 190

B.4.2 Plume Tracking . . . . . . . . . . . . . . . . . . . 191

B.5 Plume Simulation Environment . . . . . . . . . . . . . . . . . . . . 193

B.5.1 Modeling a Plume . . . . . . . . . . . . . . . . . . . . . . . 194

B.5.2 Sampling a Plume . . . . . . . . . . . . . . . . . . . 195

B.5.3 Reconstructing a Plume from AUV Sample Points . . . . . . . . . . . . . . 196

B.5.4 Results . . . . . . . . . . . . . . . . . . . . . . . . . . 197

B.6 Forecasting Long Term Variations … . . . . . . . . . . . . . . . . . . . 199

B.7 Looking Ahead . . . . . . . . . . . . . . . . . . . . 201

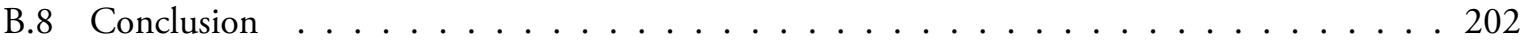




\section{Chapter 1}

\section{Introduction}

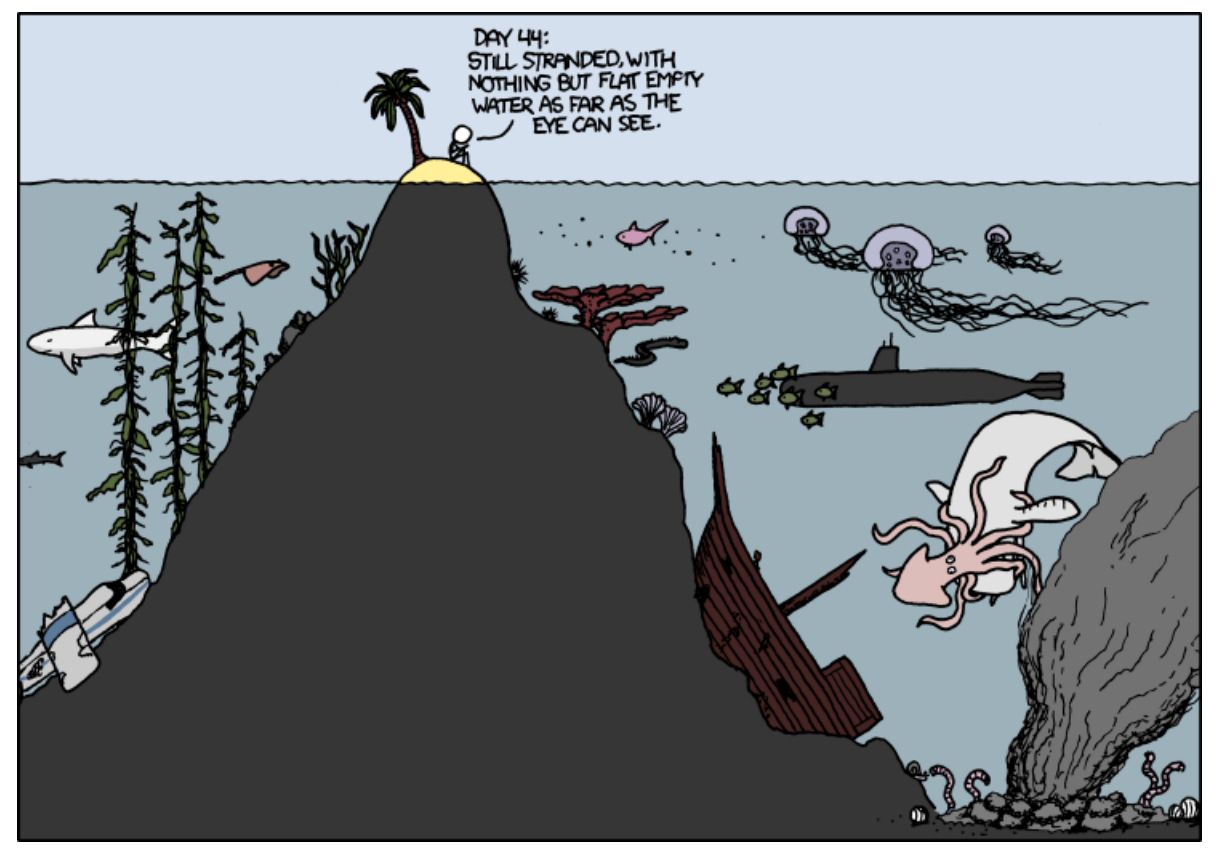

“Telescopes and bathyscaphes and sonar probes of Scottish lakes, Tacoma Narrows bridge collapse explained with abstract phase-space maps, some x-ray slides, a music score, Minard's Napoleonic war: the most exciting new frontier is charting what's already here."

http://xkcd.com/731/

Humans have used both sophisticated and simple instruments to help them understand and navigate the oceans for hundreds (if not thousands) of years. The taffrail log simply measured a ship's speed through water. Soundings and sea floor materials were sampled from ships using a slug of lead on a string. The compass told sailors which direction they were heading, and the sextant helped them determine position. Not to mention 
world maps were made from these measurements to further aid navigation. The past hundred years have seen many new oceanographic instruments developed as new materials became available and scientific interest in the ocean has greatly increased. Within the past fifty years, many of the original simple oceanographic technologies have been revisited and redesigned, and new technologies have emerged with the rapid and ongoing development of computer technology. Oceanographers and ocean engineers are always looking to improve ocean sampling technologies and systems, and are especially motivated by the fact that going to sea on a research vessel is expensive (tens of thousands of dollars per day). The expense limits time for data collection, often only allowing for one or two opportunities to collect a desired data set. If a data set is collected, but does not capture the feature or phenomenon the oceanographer requires, that is a costly misdirection of resources. That the limited data collected may still be of use to oceanographers researching this or some other subject is only a partial consolation.

Today, a number of groups are working on the problem of intelligent oceanographic sampling methods. One approach uses oceanographic data models and forecasts based on physics and/or previously collected data sets from a region to predict the location of an oceanographic feature of interest and attempt to sample an area accordingly with ship-based, moored, free-floating, or free-swimming instruments. Another approach is to use the developing technology of sensor-equipped unmanned underwater vehicles and/or unmanned surface vehicles running real-time intelligent autonomy algorithms to adapt the vehicle's motion to changes in the environment, effectively seeking out and tracking an oceanographic feature of interest with very little or no previous knowledge of the ocean environment. Both of these methods result in adaptive sampling of the ocean environment. The focus of this thesis is the latter, aiming for more efficient and intelligent ocean sampling strategies by way of cutting-edge underwater vehicle technology and onboard autonomy systems.

\subsection{Concepts/Approach}

The goal of this work is to develop a system for the tracking of hydrographic features using autonomous underwater vehicles (AUVs). This tracking is done

- adaptively to account for the dynamic nature of hydrographic features,

- collaboratively between AUVs (and other marine platforms) to collect more complete data sets for feature detection, and

- autonomously such that the AUVs determine the spatiotemporal positions or boundaries of the features, to efficiently detect and track the features with as little human intervention as possible. 
The motivation for this research stems from the need to sample the ocean in an increasingly low-cost, efficient, and intelligent manner such that scientists can predictively gather the data sets they need within a minimum of time aboard a research vessel. The use of AUVs with collaborative and adaptive autonomy also allows for measurements (environmental and otherwise) to be taken over a 3D area in space while still measuring time-dynamic properties. This gives a much greater probability of synoptic data coverage in the selected area of the ocean than using drifters, moored instruments, towed instruments, or AUVs without the ability to collaborate and adapt to changes in the environment.

The field of marine autonomy on unmanned underwater vehicles is advancing quickly, and the next step is to have the AUVs adapt their motion to the features of the underwater environment in real time, without guidance from an operator.

This thesis examines the methodology for performing autonomous and adaptive oceanographic feature tracking on board (solo and multiple) AUVs, addressing AUV autonomy, multi-AUV communication, and feature detection and tracking strategies. An emphasis is placed on the autonomous and adaptive coordinated sampling and tracking of four types of hydrographic features using AUVs: thermoclines, internal waves, and underwater fronts and plumes. The AUV autonomy system used here is comprised of the Mission Oriented Operating Suite (MOOS) and the Interval Programming (IvP) Helm [1,2]. The method of real-time underwater communication is assumed to be via acoustics, though other types of communication are considered when the AUV is on the sea surface. The importance of synoptic sampling based on the characteristic spatiotemporal scales of ocean features is discussed, and the types of AUVs best suited for various scales of feature-tracking experiments are evaluated.

\subsection{Thesis Outline \& Contributions}

The original research contributions of this thesis comprise part of Chapter 2, all of Chapters 3, 4, 5, \& 6, and the Appendices.

\section{Chapter 2: Background}

This chapter provides the technical and oceanographic background for the thesis. It explains the concept of environmentally adaptive ocean sampling, some of the available AUV technologies, the challenges of working with AUVs in the ocean environment, and the concept of characteristic spatiotemporal scales of oceanographic features. This chapter also looks at past and current methods of oceanographic feature tracking from the literature to further motivate the work in this thesis. 


\section{Chapter 3: Thermocline Tracking: A Proof-of-Concept for Autonomous Adaptive Environ- mental Assessment and Feature Tracking}

This chapter introduces the concept of Autonomous and Adaptive Environmental Assessment (AAEA) of oceanographic features using AUVs. This concept is then applied to thermocline tracking with AUVs as a proof-of-concept taken from theory to implementation. Thermocline tracking results are given for multiple field experiments.

\section{Chapter 4: Internal Wave Detection Experiment}

This chapter describes the Internal Wave Detection Experiment in the Tyrrhenian Sea in 2010. This experiment was designed to showcase the use of autonomous and adaptive thermocline tracking coupled with multiple autonomously coordinated AUVs to capture the signals of any passing internal waves. The results characterizing the detected internal waves are also presented.

\section{Chapter 5: Front Tracking}

This chapter explores autonomously and adaptively detecting and tracking underwater fronts in detail. These features are often significantly complex and dynamic in both horizontal space and time. 2D, 3D, and multiAUV front tracking behaviors that have been developed for this work and tested in virtual experiments are described, and results from the tests in a simulated Multidisciplinary Simulation, Estimation, and Assimilation Systems (MSEAS) ocean model environment of the Mid-Atlantic Bight region are presented.

\section{Chapter 6: Plume Tracking}

This chapter explores concepts and approaches for autonomously and adaptively detecting and tracking various types of underwater plumes.

\section{Chapter 7: Conclusion \& Future Directions}

The final chapter summarizes the contributions of this thesis and briefly explores the direction of future work on this subject.

\section{Appendix A: MSEAS Integration}

Appendix A details the integration of Massachusetts Institute of Technology (MIT) MSEAS ocean models into the MIT LAMSS (Laboratory for Autonomous Marine Sensing Systems) AUV virtual experiment 
environment and the associated GEOV (Google Earth interface for Ocean Vehicles) topside display. The MSEAS-LAMSS interface is described, as well as the interface for the MSEAS environmental display (overlay) in Google Earth and the topside CTD display.

\section{Appendix B: Constructing a Distributed AUV Network for Underwater Plume-Tracking Op- erations}

Appendix B presents original research conducted as an initial foray into a plume boundary parametrization and tracking method for AUVs. 


\section{Chapter 2}

\section{Background}

This chapter provides the technical and oceanographic background and motivation for the research presented in the body of the thesis. This chapter is divided into five sections: an introduction to adaptive sampling, a description of some of the challenges of working with AUVs in the ocean environment, an explanation of some of the available AUV technologies, an explanation of the concept of characteristic spatiotemporal scales of oceanographic features, and a literature review motivating the need for autonomous and adaptive approaches to feature tracking using AUVs.

\subsection{Autonomous Adaptive Sampling}

The adaptive sampling methods that are applied in this thesis result in targeted observations of the ocean environment where a feature of interest is present. Adaptive sampling for this particular application is a twostep process that includes initially assessing (surveying and analyzing) the local environment to determine the presence of an oceanographic feature and subsequently tracking that feature over space and time to maintain

Portions of this chapter are (C2010 IEEE. Reprinted, with permission, from S. Petillo, A. Balasuriya, and H. Schmidt, "Autonomous Adaptive Environmental Assessment and Feature Tracking via Autonomous Underwater Vehicles," Proceedings of OCEANS 2010 IEEE - Sydney. [3]

Portions of this chapter are (C2012 Stephanie Petillo et al. Reprinted, with permission, from S. Petillo, H. Schmidt, and A. Balasuriya, "Constructing a Distributed AUV Network for Underwater Plume-Tracking Operations," International Journal of Distributed Sensor Networks: Special Issue on Distributed Mobile Sensor Networks for Hazardous Applications. [4]

Portions of this chapter are (2010 IFAC. Reprinted, with permission, from S. Petillo and H. Schmidt, "Autonomous and Adaptive Plume Detection and Tracking with AUVs: Concepts, Methods, and Available Technology," Proceedings of the 9th IFAC Conference on Manoeuvring and Control of Marine Craft. [5] 
focused sampling in the region of study. The motivation behind adaptive sampling is to increase the efficiency of sampling and the synopticity of the collected data. This research focuses on the use of AUVs with onboard autonomy systems and environmental sensors to adaptively sample the ocean environment at and around features of interest using the aforementioned two-step process in real time. This results in a feedback loop involving environmental sensor readings, onboard data processing, and the autonomy system updating the desired motion of the AUV to adapt to and track the motion of a dynamic ocean feature to collect more data. This concept is sketched in Fig. 2-1 and applied in the body of this work.

\section{Thermocline Tracking}
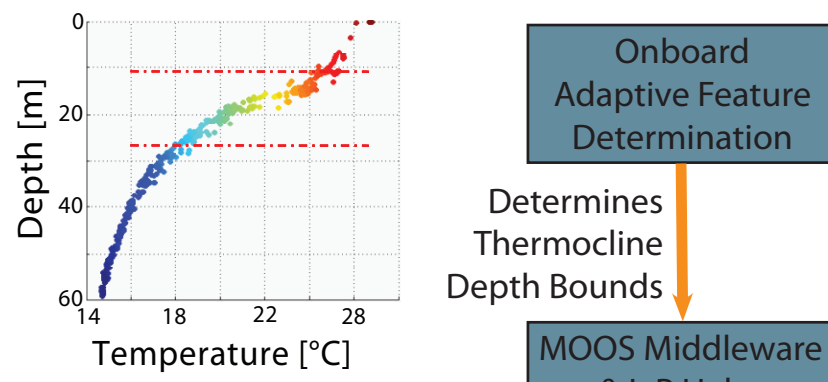

Determines
Thermocline

Depth Bounds

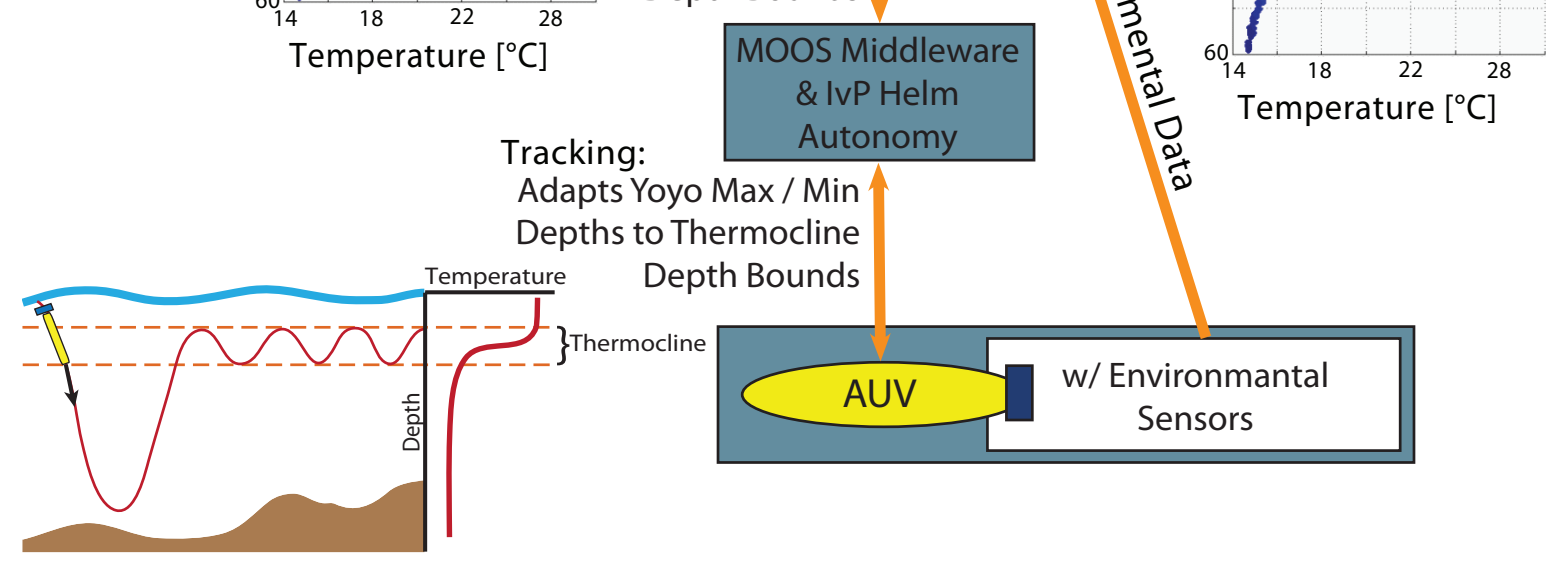

Figure 2-1: A conceptual sketch of an adaptive sampling feedback loop on board an autonomously-guided AUV carrying environmental sensors. The feature of interest in this example is a thermocline. Autonomous and adaptive thermocline tracking with AUVs is described in detail in Chapter 3.

\subsection{Advantages \& Challenges}

\subsubsection{AUVs in the real environment}

When implementing any autonomy processes, such as feature detection and tracking, on board AUVs, it is vital to the success of the mission (and life of the vehicle) to account for the physical limitations of the AUV. A number of these constraints are described below.

- Dive limit 
- All AUVs have a depth rating. These range from about $200 \mathrm{~m}$ for a coastal AUV to about 2000 $\mathrm{m}$ for a deep-rated AUV. This ultimately restricts how deep an AUV can dive.

- Surface obstacles

- AUVs on or just below the surface are not easily visible to surface craft such as ships and boats, making AUVs vulnerable to collisions at shallow depths.

- Daytime operations

- Since it is both difficult and dangerous to operate AUVs and deployment/retrieval equipment in the dark, we are restricted to operating AUVs during the daylight hours. Also, a typical (actively propelled) AUV only has a battery life of about 5 to 8 hours, which must be charged or replaced overnight.

- Ocean acoustics restrict the AUV to accessing only data collected on board

- The ocean environment attenuates high-frequency sound waves over a much shorter distance than low-frequency sound waves. This restricts any acoustic communications between the AUV and ship to lower frequencies to increase transmission range at the cost of bandwidth. Thus, only a minimal amount of data may be transmitted to and from the AUVs through the water. Sending higher bandwidths of data a reasonable distance $(\mathrm{O}(500 \mathrm{~m}))$ through the water (which is trivial in air using RF (radio frequency) technology) requires significantly more power underwater than in air, making it infeasible to power such an acoustic source on an AUV.

- Memory and processing time

- Each AUV must store logs of all the missions of a given day (or experiment) on board, consuming a few gigabytes of memory at a time. These small quantities add up over time, so to avoid accidentally filling a hard drive it is important not to store more data than necessary for on-board computations. This means that we cannot store satellite data or large ocean models on board the vehicle. In addition, since most data processing occurs on board the AUV in near real time, it is important that no one piece of code, algorithm, or process takes more than fractions of a second at a time. 


\subsubsection{AUV Networks}

An AUV network allows for the dynamic interaction of multiple AUVs to better adapt to dynamic features in the marine environment. That is, a network of AUVs has the ability to distribute its nodes around the entire feature and move with the feature, whereas a solo AUV may be optimally placed for sampling within a feature but could not determine the horizontal spatial extent of a feature and track it simultaneously on its own. Using the estimated characteristic scales of the feature (from satellite imagery, past surveys, or physics-based calculations) in guiding the AUV autonomy behaviors, a network of AUVs can be distributed in space and time to detect and track the feature and avoid aliasing the data. This desire for adaptive feature tracking also underscores the necessity for using mobile (self-propelled) sensing platforms instead of, or in conjunction with, fixed and drifting sensing platforms (e.g., buoys, Argo floats) such that sampling is performed more efficiently (minimizing overlapping data) and the researcher can be certain that a complete data set describing the feature has been captured.

\subsubsection{Environmentally Adaptive Autonomy \& Autonomous Coordinated Control}

The decision-making system behind coordinating a sophisticated network of AUVs for feature tracking is the underlying autonomy system that must run on board each AUV. An autonomy system, such as that described in Section 2.3, allows an AUV to adapt to its environment in near real time, without human intervention. A few of the minimum requirements of using and interacting with a robust autonomy system are inter-AUV (acoustic) communications, support for adaptive autonomy behaviors (supplied by the user) to be executed by the AUVs, and an intelligent (autonomous) means of deciding which behaviors have priority during a given mission. A tiered mission planning structure for this system is proposed in which the large-scale, overall mission drives the selection of the formation of the AUVs (via multi-AUV coordinated autonomy behaviors) and allows each AUV to use individual autonomy behaviors to follow the feature within its local vicinity. Position and minimal environmental data products are exchanged acoustically across the AUV network to update the feature model (or parametrization) and, subsequently, the local missions of the AUVs. This creates a feedback loop using the processed and exchanged data as inputs for updating the large-scale mission, then the local missions, to collect, exchange, and reprocess more data between AUVs. This loop continues for as long as required by the researcher/user. 


\subsubsection{Acoustic Communication}

One of the primary challenges using multiple AUVs and other networked nodes simultaneously in the underwater environment is that of communication. Electromagnetic waves at the wavelengths feasible for useful AUV communication are quickly attenuated in water within a few meters of the surface, leaving acoustics as the primary method of real-time underwater communication. Until now, there have been few (if any) options for intelligent multi-AUV ( $>2$ AUVs) acoustic communication schemes, though the Goby underwater communication and autonomy project (version 2.0) strives to remedy the need for coordinated message queuing and passing between multiple AUVs [6,7]. This allows each AUV to communicate with neighboring AUVs and share data and knowledge with the sensing platforms in its underwater network.

It is important to note, however, that many features are dynamic in the mesoscale or larger, and AUVto-AUV and AUV-to-ship/lab acoustic communication (at least in the public domain and on power-limited AUVs) is only possible up to a range of about $10 \mathrm{~km}$. Our group at MIT has found that our equipment is usually limited to about $2 \mathrm{~km}$ of acoustic communication range in the coastal ocean and lake environments where most of our experiments have been performed recently. Our Bluefin 21" AUVs and lab setup, which are each equipped with a WHOI (Woods Hole Oceanographic Institution) Micro-modem and model WHBT-2 $28 \mathrm{kHz}$ transducer, transmit data in the frequency band of $23-27 \mathrm{kHz}$, centered around $25 \mathrm{kHz}[8]$. There are two realistic solutions to the acoustic communication range restriction we experience. The first and more complex solution is to implement a multi-hop acoustic communication scheme in which data from one AUV is passed down through a chain of AUVs to its destination. This is time consuming due to the nature of sending and listening for transmitted data packets one at a time between communicating AUVs. Given that AUVs will often be hundreds of meters apart or more and sound speed propagation is about $1500 \mathrm{~m} / \mathrm{s}$ in the ocean, data packets take an observable amount of time to transmit through the water $(\mathrm{O}(1 \mathrm{sec}))$. This method would also require extensive research into data routing on dynamic and time-scheduled messaging networks. The second and more immediately feasible (potentially more reliable) solution would be to restrict communication of large environmental data sets to RF or satellite methods while an AUV is on the surface and use a delay-tolerant network rescheduling scheme. Although this method removes much of the real-time underwater data passing between AUVs (with the exception of basic position updates of nearby AUVs for avoiding collisions), it would take a large burden off of the acoustic channel and still allow each AUV to be deployed based on the most current overall picture of the feature while still performing solo autonomous and

adaptive feature tracking in its local vicinity in real time. Periodic surface communication would work best in the case that the AUVs can surface with great enough frequency (within the characteristic time scale of 
the feature) to be re-directed to a more optimal sampling position, but with low enough frequency that the feature tracking mission is not significantly disrupted by the AUV taking the time to come to the surface more often.

For the multi-AUV adaptive feature tracking missions presented in this thesis, there is (intentionally) a minimal amount of processed environmental data passed between a small number of AUVs, avoiding the need for multiple acoustic hops over the network or surfacing to transmit data.

\subsubsection{Data Fusion}

The fusion of data both from multiple sensors on a single AUV and all sensors across all networked AUVs is crucial to the success of coherently adapting a fleet of AUVs to track an ocean feature and collect a synoptic data set. When fusing data from a single vehicle, the largest concerns are keeping all data accurately timeand position-stamped. Across multiple AUVs, the data must also be quality-checked for corruption during transmission after passing it from one vehicle to the next. It is proposed that on board each AUV the computer must mesh the data sets from all AUVs into a single data set, sorted over the times and positions at which each data point was taken, for each variable (i.e., temperature, salinity, etc.). Upon processing of these data on board (as on-board processing is the only way to adapt to a dynamic environment in real time), for each variable, probability weighting functions over time and space must be applied to each data point based on the characteristic spatiotemporal scales of that variable. A basic Gaussian-shaped weighting function would ideally be used for this task, but the simpler linear weighting used in this work is also sufficient. This will associate, say, all temperature readings taken in the last few minutes and within a radius of a kilometer horizontally (assuming the AUV can resolve its position with even better accuracy), but will ignore any temperature readings that fall outside of these ranges as independent from those inside. This essentially creates an overlap of data within a radius of one standard deviation about the sample point, as sketched in Fig. 2-2, that can be used to maintain synoptic sampling in a data set. This data fusion method could be implemented using an SQLite [9] (or similar) database on each AUV to compound and sort all of the environmental data from all AUVs, which may then be processed in a mathematics program such as MATLAB [10] or Octave [11], or by a simple $\mathrm{C}++$ [12] parser with algorithms utilizing $\mathrm{C}++$ vector math libraries. This is similar to creating an evidence grid of the AUVs' environmental data [13]. The resulting ocean environment reconstructed through data fusion with weighting can guide the mission planning for a fleet of AUVs tasked to track a feature. The AUVs can survey an area with high enough resolution to find the feature, approximate the feature's shape with higher weighting near the actual sample points, and revise their coordinated survey strategy based on 
this new estimate of the feature's position.

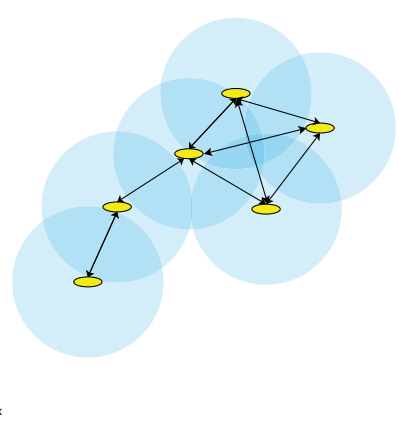

Figure 2-2: Blue circles around AUV sample points represent the range of significant data association possible (the radius of standard deviation of the Gaussian distribution). For any two AUV samples with overlapping range circles, an arrow is drawn to represent the fusion of data between those positions, which may be used to construct a larger-scale ocean data model when chains of fused data are combined to form a web of unaliased (see Section 2.4.1) connections.

\subsection{AUV technologies}

\subsubsection{Autonomy Middleware}

There are a number of autonomy architectures suitable for use on board AUVs. Of these, the Mission Oriented Operating Suite (MOOS) [2], the Robot Operating System (ROS) [14], and the Lightweight Communications and Marshalling (LCM) library [15] are the most well known and widely used in the marine autonomy community. There are benefits and drawbacks to all of these systems that are beyond the scope of this thesis. This thesis will only focus on autonomy implementations using MOOS, as that is how the AUVs used in this work have been configured for both virtual and field experiments.

\section{MOOS \& IvP Helm}

The autonomy system used on board the AUVs for all field and simulated work described in this thesis is MOOS. When conducting field experiments with AUVs (usually only 1 or 2) in the water, MOOS is the underlying autonomy system on board the AUVs and on the topside mission-command computer. MOOS provides a publish-subscribe architecture that essentially deals with information sharing between autonomy processes and behaviors on board each AUV, as well as through the water between the AUVs and the topside 
computer [1]. To add some intelligence to the system, the IvP (Interval Programming) Helm multi-objective optimization engine is used in conjunction with MOOS to implement the use of autonomy behaviors (e.g., vertical yo-yos, trail-an-AUV, horizontal racetracks, safety behaviors) on the AUVs [1,2]. Each behavior run by the IvP Helm generates a single objective function competing for the AUV's desired speed, heading, and depth. The design of MOOS-IvP autonomy also allows AUV operators to write plug-and-play code (processes and behaviors), significantly easing implementation.

\subsubsection{Acoustic Communications}

Acoustic communications (acomms) are the primary form of communications between the ship and the AUVs. The ship receives status and data updates from the vehicle every couple of minutes through acomms while the vehicle is under water. This allows for near real-time monitoring of the AUV throughout a mission.

Messaging via acomms is handled through the Goby (version 2.0) autonomy software on all platforms, where the Goby software schedules the transmissions of each node (AUVs, communication buoys, topside operator, etc.) in the network [6,7]. Goby encodes data on one node, initializes the data transmission through the acoustic channel, and then decodes the data when they are received on another node.

These two essential pieces to our AUV network (Sections 2.3.1 and 2.3.2) allow our AUVs to adapt their motion based on sensor readings, without a human in the loop (but while being monitored in real time by an AUV operator). This allows for ocean feature detection and tracking by AUVs to occur both autonomously and adaptively in real time and across multiple AUVs.

\subsubsection{Sensors \& Instrumentation}

\section{Oceanographic Sensors}

There are a large number of sensors that can be mounted on AUVs of various types. Some sensors are specially designed to mount on specific AUVs, some are off-the-shelf for use with AUVs, and some must be retrofitted. Oftentimes, the mounting of a sensor will require modifications to the AUV body and/or electronics. Sensors also usually need to be interfaced to the software on the AUV in order for the data to be collected, though some have stand-alone data loggers. The work presented in this thesis requires that an AUV collect and process the sensor data on board in real time for the AUV to autonomously and adaptively detect and track oceanographic features.

Table 2.1 lists a variety of sensors that have been mounted on AUVs in the past and the environmental characteristics that they measure. 
Table 2.1: Features, their measurable tracers, and associated instrumentation

\begin{tabular}{|c|c|c|}
\hline Features/Obesrvations & Measurable Tracers & Instruments \\
\hline $\begin{array}{l}\text { Thermocline, halocline, pycno- } \\
\text { cline, sound speed }\end{array}$ & $\begin{array}{l}\text { Temperature, conductivity, pres- } \\
\text { sure }\end{array}$ & $\begin{array}{l}\text { CTD } \quad \text { (Conductivity- } \\
\text { Temperature-Depth) [16] }\end{array}$ \\
\hline $\mathrm{O}_{2}$ concentration & $\begin{array}{l}\text { Partial pressure of } \mathrm{O}_{2} \text { in water } \\
\text { (via temperature \& conuctivity) }\end{array}$ & Dissolved Oxygen sensor [17] \\
\hline $\begin{array}{l}\text { Phytoplankton biomass \& } \mathrm{Cl} \\
\text { concentration }\end{array}$ & Chlorophyll-a fluorescence & Fluorometer [18] \\
\hline Light attenuation & $\begin{array}{l}\text { Photosynthetically Active Radi- } \\
\text { ation (PAR) of } 400-700 \mathrm{~nm} \\
\text { wavelength }\end{array}$ & PAR sensor [19] \\
\hline Currents & $\begin{array}{l}\text { Doppler (frequency) shift of } \\
\text { sound waves }\end{array}$ & $\begin{array}{l}\text { ADCP (Acoustic Doppler Cur- } \\
\text { rent Profiler) [20] }\end{array}$ \\
\hline Fronts & $\begin{array}{l}\text { Temperature, conductivity, pres- } \\
\text { sure, Doppler shift }\end{array}$ & CTD, ADCP \\
\hline $\begin{array}{l}\text { Hydrothermal Vent Plume or } \\
\text { Source }\end{array}$ & Temperature anomaly & CTD \\
\hline $\begin{array}{l}\text { Hydrothermal Vent Plume or } \\
\text { Source }\end{array}$ & Particle content & $\begin{array}{l}\text { Optical sensors: transmissome- } \\
\text { ter, nephelometer }\end{array}$ \\
\hline $\begin{array}{l}\text { Hydrothermal Vent Plume or } \\
\text { Source }\end{array}$ & Chemical tracers & $\begin{array}{l}\text { Optical sensors: SUAVE (Sys- } \\
\text { tem Used to Assess Vented Emis- } \\
\text { sions), ZAPS (Zero Angle Pho- } \\
\text { ton Spectrophotometer), eH (re- } \\
\text { dox potential) }\end{array}$ \\
\hline Hydrothermal Vent Source & Water velocity & $\begin{array}{l}\text { Acoustic sensors: ADCP, sides- } \\
\text { can sonar, multibeam sonar }\end{array}$ \\
\hline Hydrothermal Vents Source & Bathymetry & $\begin{array}{l}\text { Multibeam mapping sonar, cam- } \\
\text { era (still or video) }\end{array}$ \\
\hline
\end{tabular}

Of these, conductivity-temperature (CT) and conductivity-temperature-depth (CTD) sensors are two of the most commonly used sensors for oceanographic sampling using AUVs. The work presented in this thesis relies heavily on CT measurements (coupled with a pressure sensor for depth measurements) to guide the motion of the AUVs when feature tracking, though the CT or CTD is by no means the only sensor that feature tracking techniques could employ.

\section{Navigation \& Communication Instrumentation}

There are also a large number of other instruments that may be used on AUVs to perform the basic and necessary functions of navigation and communication. These include (but are not limited to) compasses, GPS 
units, RF communication hardware (Wi-Fi, Freewave, etc.), hydrophones and acoustic transducers for acoustic communication and navigation, inertial measurement units (IMUs), inertial navigation units (INUs), Doppler velocity logs (DVLs), depth (pressure) sensors, and Iridium phone hardware for satellite-based communication.

The AUVs used for most of the work in this thesis have had some of their basic instrumentation updated over the past few years, but through most of the field experiments for this work (2009-2010), it remained largely unchanged. The instrumentation and sensor configuration for both the MIT Bluefin 21" Unicorn AUV and the NURC OEX Harpo AUV used in field experiments in 2009 and 2010 are described in Chapter 4, Section 4.5.1.

\subsubsection{AUV Types}

In this section the abilities and traits of a variety of AUVs are classified. Although this is not a thorough classification of all AUVs, since there are many different commercial and made-in-house AUVs in the ocean community today, a number of AUVs are generalized into categories to allow them to be compared.

The most basic attributes to look at when comparing AUVs are speed, deployment duration (battery life), propulsion (active or passive), range of motion control, depth rating, navigation method, communication, hotel power load on board, autonomy system, hull shape, ease of retrofitting sensors, and what sensors it carries 'off the shelf'. See Table 2.2.

Some examples of the AUVs that fall into the three categories in Table 2.2 are listed below.

Gliders:

- Slocum gliders (thermal and electric) from Teledyne Webb Research [21]

- Spray gliders developed under ONR support by Scripps and Woods Hole Oceanographic Institution (WHOI) scientists [22]

- Exocetus Coastal Glider from Exocetus Development, LLC (formerly ANT Littoral gliders developed under ONR by ANT, LLC) [23]

- Seagliders from the Applied Physics Laboratory - University of Washington, iRobot, and Kongsberg Maritime [24-27]

Actively propelled, torpedo shaped AUVs:

- Bluefin 9", 12", and 21" from Bluefin Robotics [28] 
Table 2.2: Attributes of various types of AUVs

\begin{tabular}{|c|c|c|c|}
\hline Attribute & Glider & $\begin{array}{l}\text { Actively propelled, } \\
\text { torpedo shaped }\end{array}$ & $\begin{array}{l}\text { Actively propelled, not } \\
\text { torpedo shaped }\end{array}$ \\
\hline Speed & $0.0-0.5 \mathrm{~m} / \mathrm{s}$ & $0.0-3.0 \mathrm{~m} / \mathrm{s}$ & $0.0-3.0 \mathrm{~m} / \mathrm{s}$ \\
\hline Duration & weeks to months & hours to days & hours to days or weeks \\
\hline Propulsion & passive & active & active \\
\hline Vertical motion & constant yoyo & $\begin{array}{l}\text { unrestrained (but most } \\
\text { do not hover) }\end{array}$ & $\begin{array}{l}\text { unrestrained (some } \\
\text { hover) }\end{array}$ \\
\hline Horizontal motion & unrestrained & unrestrained & unrestrained \\
\hline Depth rating & $\begin{array}{l}\text { most }<2 \mathrm{~km} \text {, one up to } \\
6 \mathrm{~km}\end{array}$ & up to $6 \mathrm{~km}$ & up to $6 \mathrm{~km}$ \\
\hline Navigation & $\begin{array}{l}\text { dead reckoning (DR), } \\
\text { compass, GPS }\end{array}$ & $\begin{array}{l}\text { IMU (inertial } \\
\text { measurement unit), } \\
\text { acoustics, DR, compass, } \\
\text { GPS }\end{array}$ & $\begin{array}{l}\text { IMU, acoustics, DR, } \\
\text { compass, GPS }\end{array}$ \\
\hline $\begin{array}{l}\text { Communication } \\
\text { method }\end{array}$ & at surface (Iridium, RF) & $\begin{array}{l}\text { at surface (Iridium, RF), } \\
\text { underwater (acoustic) }\end{array}$ & $\begin{array}{l}\text { at surface (Iridium, RF), } \\
\text { and/or underwater } \\
\text { (acoustic) }\end{array}$ \\
\hline Hotel load & $<10$ Watts & $<100$ Watts & $<100$ Watts \\
\hline Autonomy & $\begin{array}{l}\text { possible, not fully } \\
\text { implemented }\end{array}$ & implemented frequently & implemented frequently \\
\hline Shape & torpedo with wings & torpedo & $\begin{array}{l}\text { non-torpedo, may be } \\
\text { multi-hull }\end{array}$ \\
\hline Typical sensors & $\begin{array}{l}\text { CTD (or CT), pressure, } \\
\text { bottom ranger, compass }\end{array}$ & $\begin{array}{l}\text { CTD (or CT), pressure, } \\
\text { sidescan sonar, acoustic } \\
\text { transducer (for } \\
\text { communication), } \\
\text { compass }\end{array}$ & $\begin{array}{l}\text { varies widely; pressure, } \\
\text { acoustic transducer (for } \\
\text { communication), } \\
\text { compass }\end{array}$ \\
\hline
\end{tabular}

- Ocean Explorer (OEX) from Florida Atlantic University, operated by the Centre for Maritime Research and Experimentation (formerly NATO Undersea Research Centre), Italy [29]

- REMUS from WHOI and Hydroid-Kongsberg Maritime [30,31]

- Iver from Ocean Server [32]

- Folaga from Graal Tech (more like a hybrid glider-but-actively-actuated, torpedo-shaped AUV) [33]

Actively propelled, not torpedo shaped AUVs:

- Sentry and Autonomous Benthic Explorer $(A B E)$ from Woods Hole Oceanographic Institution (WHOI) $[34,35]$

- Puma, Jaguar, and SeaBED-class from WHOI [36] 
- Odyssey IV Class from Massachusetts Institute of Technology’s Sea Grant AUV Laboratory [37]

For the work in this thesis, the MIT Bluefin 21" Unicorn AUV, the NURC OEX Harpo AUV, and the NUWC Iver 2 Hammerhead AUV were employed. The AUV requirements were the following:

- Active propulsion

- On-board autonomy (MOOS and IvP Helm)

- CT and pressure, or CTD, sensor(s)

- Acoustic communications using the WHOI MicroModem and WH-BT-2 $28 \mathrm{kHz}$ acoustic transducers

- GPS positioning when on the surface

- DVL positioning underwater, or at least Dead Reckoning (DR) algorithms

Specific hardware found on these platforms are described in Chapter 4, Section 4.5.1, and Chapter 3, Section 3.7.

\subsection{Spatiotemporal Scales of Ocean Features}

For this work, it is not only important to know the spatial and temporal scales on which AUVs operate, but also to know the scales at which any oceanographic features of interest occur. With these two pieces of knowledge, AUV types can be selected that are properly equipped to detect and track an ocean feature based on corresponding spatial and temporal coverage. This improves the chances of collecting a maximally synoptic data set.

Oceanographic features are often classified into one of three spatial scale domains based on the horizontal length scales over which they occur (since the vertical length scale is often small in comparison): small-scale $(\mathrm{O}(<10 \mathrm{~km}))$, mesoscale $(\mathrm{O}(10-100 \mathrm{~km}))$, and large-scale $(\mathrm{O}(>100 \mathrm{~km}))$. A collection of oceanographic features and their associated time and length scales are plotted in Fig. 2-3. This research mostly explores feature sampling on the mesoscale and sub-mesoscale and how AUVs can adapt their motion to a feature's dynamic behavior based solely on the AUVs' on-board sensor readings. To determine the horizontal length scale of a large-scale feature, it is useful to estimate it as the Rossby radius of deformation, $R=\sqrt{\frac{g^{\prime} H}{f}}$, where $g^{\prime}$ is reduced gravity across a density interface, $H$ is the mean water depth, and $f$ is the Coriolis parameter (twice the earth's angular velocity about its vertical axis) [38]. That is, the horizontal distance over which a 
parcel of fluid distinct in density from the surrounding fluid adjusts its spatial extent to a steady state based on the rotation of the earth, after the parcel is introduced into the system. The spatiotemporal scales may also be estimated from observations and historical data from the region of interest, which may be a more accurate method for certain fast-moving and small-scale flows that are insignificantly affected by the rotation of the earth, where $R$ is not applicable.

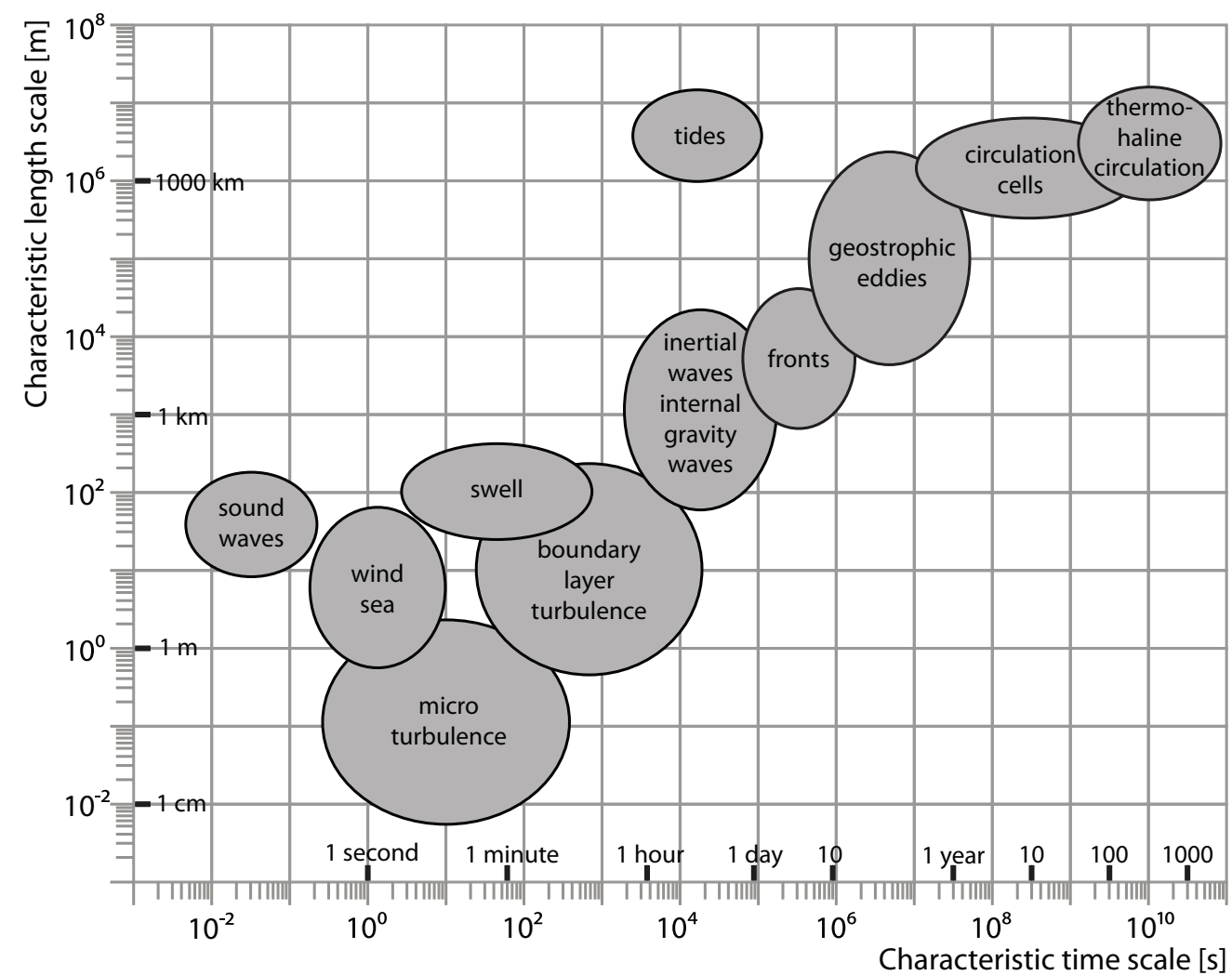

Figure 2-3: This figure depicts the characteristic horizontal length scales and time scales of various oceanographic features. Image credit: [38].

When sampling the ocean to collect data on a specific dynamic ocean feature, sampling theory from signal processing suggests that the feature should be sampled at least twice over the feature's characteristic time and spatial scales in order to be able to fully reconstruct the feature and its dynamics from the data. Thus, the temporal sampling frequency is $f_{s_{\text {time }}} \geq 2 / t_{0}$, where $t_{0}$ is the characteristic time scale. Similarly, the spatial sampling frequency is $f_{\text {space }} \geq 2 / l_{0}$, where $l_{0}$ is a characteristic length scale. This is essentially sampling at the Nyquist frequency of a feature's variations. 


\subsubsection{Synoptic Sampling vs. Spatiotemporal Aliasing}

One of the most common challenges of working with AUVs to track ocean features is that of spatiotemporal aliasing. That is, when the samples taken are too far apart in space and/or time to be able to resolve the boundaries or position of a dynamic feature at a given point in space and time. This is effectively a trade-off between data coverage and data resolution. There are two extremes here, for example:

1. A single AUV can survey a small area $(\sim \mathrm{O}(1 \mathrm{~km})$, low spatial coverage) with very high spatial sampling resolution $(>\mathrm{O}(1 \mathrm{sample} / \mathrm{m}))$ to resolve small-scale features in the water, such as pockets of turbulence. However, this survey would not have great enough coverage to determine the bounds of a $10 \mathrm{~km}$ wide algal bloom encompassing the sampling area.

2. A single AUV can survey an area once over a long time period ( $\geq O(10 \mathrm{hr})$, high temporal coverage) to sample a feature. However, it may take so long ( $>10$ hours) to perform a spatially-comprehensive survey, as witnessed by Jakuba et al. in [39], that the feature has advected away from its initial surveyed position during that period (poor temporal resolution) and the survey must be redone with less coverage to resolve the motion of the feature.

Somewhere in the middle of the above 'coverage vs. resolution' scenarios resides a delicate balance in which the characteristic scales of a dynamic feature coincide inversely with (one half) the rate at which the feature is sampled. This is essentially a sampling of the feature at its spatial and temporal Nyquist frequencies to maximize both coverage and resolution of the feature within the data set. Thus, it is necessary to know the approximate characteristic spatial and temporal scales of the feature of interest for more intelligent pathplanning purposes (see Fig. 2-4), most likely involving multiple AUVs for tracking mesoscale features that are dominantly dynamic in two or more dimensions of space, or any feature highly dynamic in time (such that an AUV moving $\leq 2 \mathrm{~m} / \mathrm{s}$ could not keep up).

The necessity for designing autonomous multi-AUV networks and implementing more intelligent and efficient feature sampling is highly motivated by this aliasing problem. Since at-sea deployments tend to be expensive and time-restricted, the ability to harness AUVs and environmentally-adaptive autonomy infras-

tructure (assuming there is access to such resources) leaves little point to deploying instruments or AUVs to map and track oceanographic features using preplanned surveys if there is no way to guarantee some amount of data synopticity in the preplanned surveys without significantly reducing survey coverage. These resources (AUVs with autonomy middleware) were available for the work presented in this thesis, thus the concept of using the characteristic scales of features to sample and track the features in the following chapters has been 


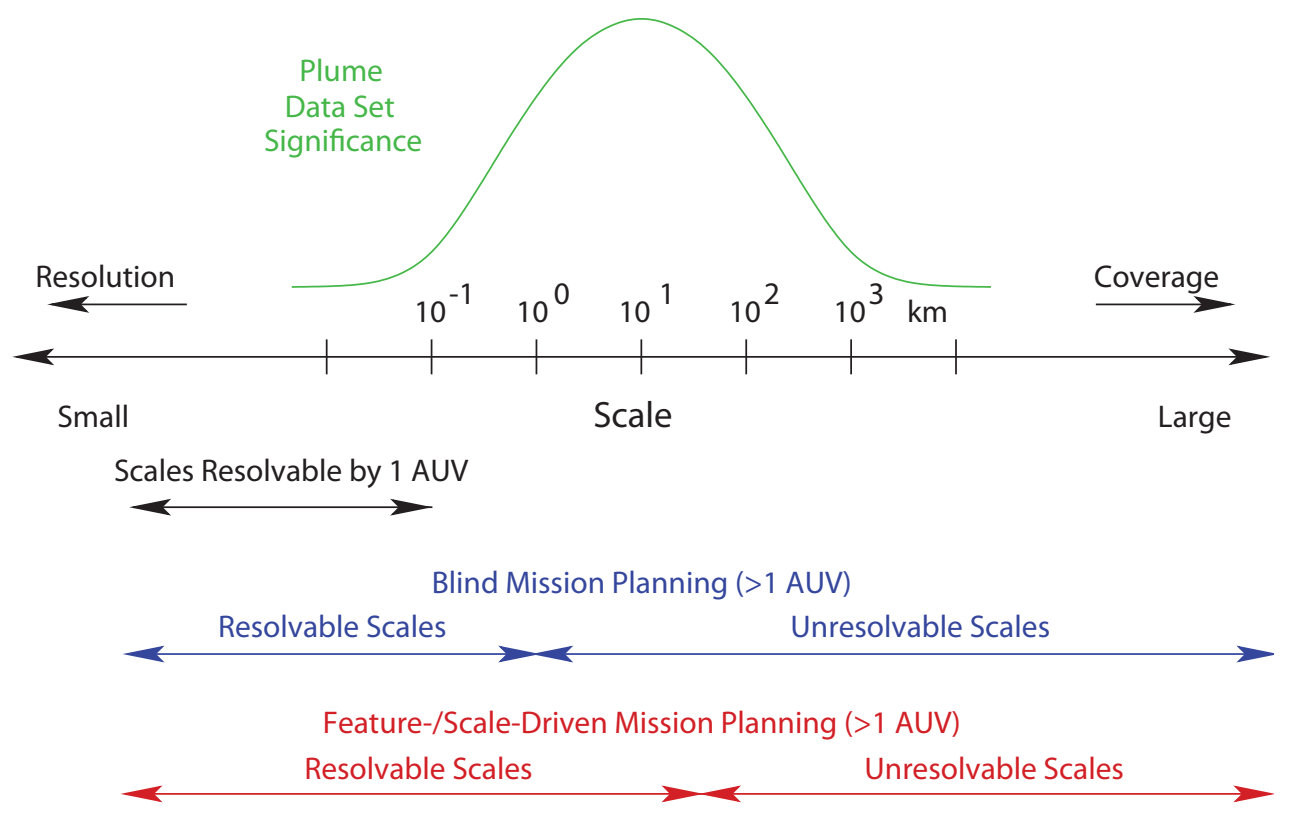

Figure 2-4: This figure depicts the characteristic length scale (in $\mathrm{km}$ ) of an $\mathrm{O}(10 \mathrm{~km})$ feature (e.g., a plume) in the horizontal plane. A similar figure can be drawn for the temporal dimension based on the characteristic time scale of a feature (with units of time). If we assume a feature has an approximate Gaussian distribution over its characteristic length scale, as shown here, we must plan AUV missions such that the collective sampling of our AUVs overlaps with the primary length scale of the feature to optimize over coverage and resolution ('feature/scale-driven' mission planning). This will improve the range of resolvable length scales in the resulting data set over that of 'blind' mission planning, especially when the AUVs' distribution is 'driven' by the characteristic spatiotemporal scales of the feature. Adapted from [40].

applied. The success (synoptic and efficient feature sampling) of the resulting AUV autonomy behaviors hinge on selecting the proper approximate characteristic spatiotemporal scales of the feature and configuring these in the autonomy behavior prior to deployment, which is an important part of the approach presented here.

\subsubsection{AUV Types and Numbers Suited to Different Features' Scales}

Knowledge of the characteristic scales and dynamics of a feature of interest is also important when deciding which type of AUV is best suited to sample or track the feature.

To pair an AUV with a type of feature that it is best suited to detect or track, consider the two primary classifications of AUVs: gliders and actively propelled AUVs. For long-duration deployments (days to months), the duration of gliders makes them the best type of AUV for the application. Multiple gliders distributed in a coordinated manner are also marginally sufficient to track mesoscale and sub-mesoscale features advected by ocean currents, since the passive propulsion and resulting slow speed of gliders through the water are directly affected by the currents as well, pushing the gliders in the same direction as the feature is advected 
(see $[41,42])$. For very deep missions that are time-dependent (achievable in or requiring short mission time, as in hours or days), involve features that are highly time-variant, or require swimming against the local currents, actively propelled AUVs are the better choice despite their shorter deployment duration. In these cases, actively propelled AUVs may be used solo, or in a coordinated fleet if a meso- or large-scale feature must be mapped as the feature advects with the changing currents. Actively propelled AUVs would also be useful in quickly surveying the extent of a sub-mesoscale feature in the horizontal plane, providing more of a snapshot of the feature. Fig. 2-5 provides a sketch of the spatiotemporal scales covered by varying numbers of gliders versus actively propelled AUVs, overlaid and underlaid with the characteristic scales of various oceanographic features.

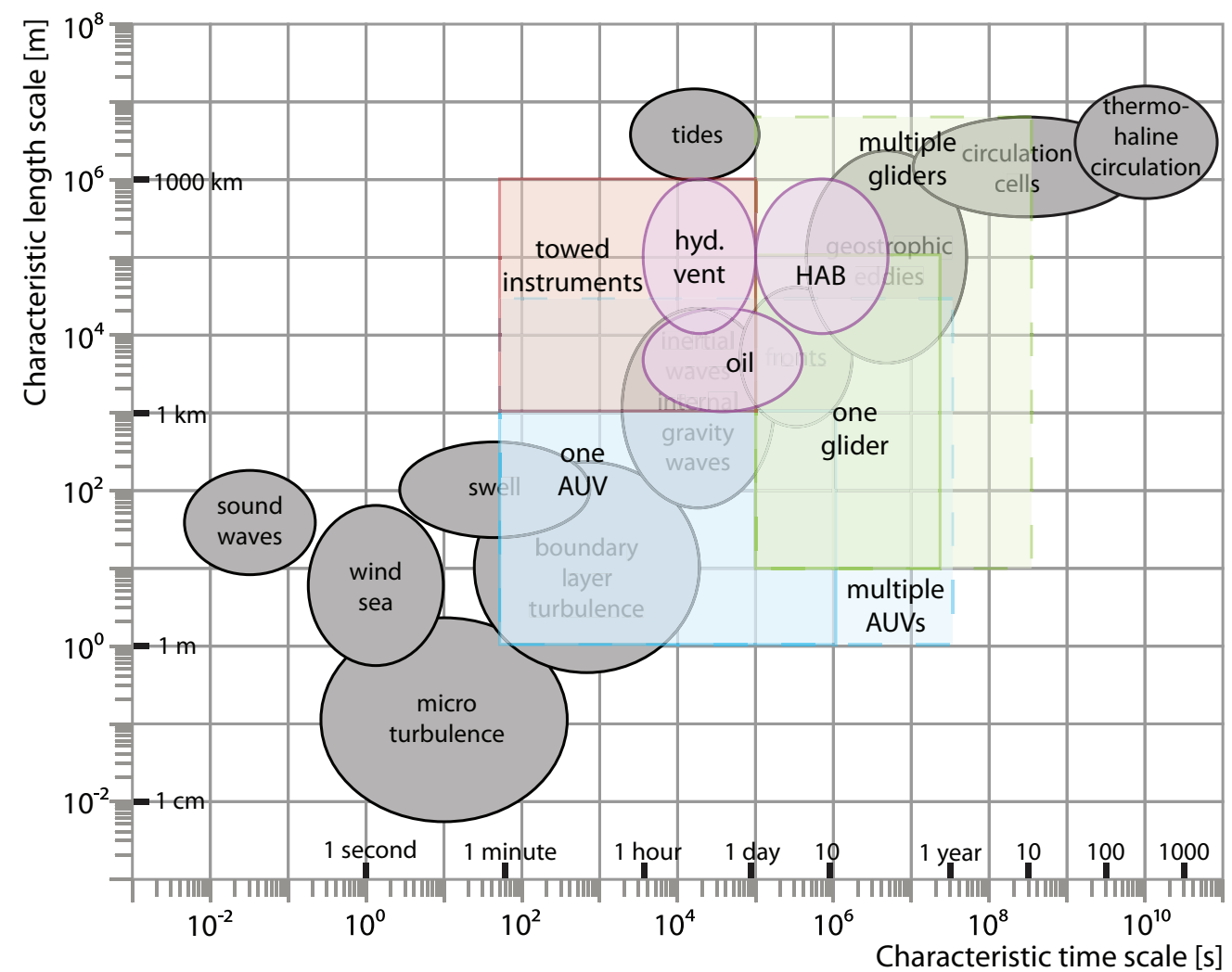

Figure 2-5: This adaptation of Fig. 2-3 shows approximate characteristic horizontal length scales and time scales (and features) that may be covered by various types and numbers of mobile underwater vehicles and moored instrumentation. In addition, the purple oval overlays show approximate scales of various types of underwater plumes, as an example of features that different platforms are best suited to detect and track. Adapted from [38].

The work in this thesis focuses on features sampled and tracked on the sub-mesoscale and mesoscale by 
one or two actively-propelled AUVs. This includes fronts, internal waves, and vertical temperature structure in coastal settings, which are ideal for sampling and tracking over time durations of days and distances of tens of kilometers or less with these vehicles. With the methods presented in the following chapters, this can be achieved without sacrificing data synopticity and while improving sampling and tracking efficiency by using environmentally-adaptive autonomy behaviors on board the AUVs to sample and track these features.

\subsection{Literature}

Before discussing the AUV feature tracking methodology that is the core of this thesis, it is important to understand the need for such environmentally-adaptive AUV autonomy in the context of the current available technologies for feature sampling and tracking. He et al. [43,44] and Carder et al. [45] summarize the need for AUV sampling technologies and methodologies best in stating that the usual moored and shipboard sampling and satellite remote sensing techniques do not have enough flexibility to sufficiently (synoptically) sample highly dynamic ocean features or features that have important signatures in the middle of the water column (below the reach of towed platforms or ROVs) or along the seafloor. These older methods are also not as cost-effective in comparison to using AUVs with preplanned surveys or even more intelligent sampling methods.

Some examples in which autonomous and adaptive feature tracking with AUVs would be an improvement over current preplanned AUV methods include: tracking a mid-water-column oil plume from a spill such as the Deepwater Horizon incident in 2010 [46], distinguishing features such as ocean convection from internal waves with a more efficient sampling pattern [47], and collecting specific data sets to be assimilated back into ocean models for improved environmental modeling and forecasting [40].

There has been a good deal of research into path planning for ocean sampling with AUVs as well, which usually requires significant prior knowledge of the environment either through an ocean model or very recent satellite imagery. The works by Yilmaz et al. [48], Hover [49], and Das et al. [42,50] provide a few examples. These methods tend to be processing-intensive and (lacking adaptive capabilities) the resulting planned paths may not sample the entire desired feature when the AUV is deployed in the actual ocean. Some of these path planning methods, however, do succeed in being adaptive in their sampling technique, but are more complex and resource-intensive than the in situ adaptive methods that are developed and evaluated in this thesis. The reason for the minimal-complexity approaches to feature tracking that are described in the body of this work is that reduced complexity in software (e.g., autonomy behaviors) often results in lower CPU load and increased robustness to the intricacies of the data being collected, processed, and reacted upon when 
the AUV is deployed in the actual ocean. This increased simplicity also makes it easier to address and correct any unforeseen problems or quirks that arise when autonomous techniques that were developed and tested in ocean model environments are deployed in the highly dynamic actual ocean for the first time.

Other groups that have put autonomous and adaptive feature tracking methods with AUVs to use will be addressed as appropriate in the following chapters. As this research is new in the field of deployable AUV autonomy for adaptive ocean sampling, it will become apparent reading this thesis that there is much room for future expansion of the methods presented here. 


\section{Chapter 3}

\section{Thermocline Tracking: A Proof-of-Concept for Autonomous Adaptive Environmental}

\section{Assessment and Feature Tracking}

\subsection{Introduction}

Underwater environments are highly dynamic and varied in space and time, posing significant challenges to the detection and tracking of hydrographic features. Often, oceanographers want to collect data for a given feature, and to do so they need to have knowledge of when and where it may occur. However, the data collected may be sparse or fail to capture the feature if it is highly dynamic. This is where Autonomous Underwater Vehicles (AUVs) are becoming more and more valuable. AUVs are frequently used to sample the ocean across a much larger depth range than possible with satellites and much more coverage than instrument casts from a ship, providing four-dimensional coverage (3D space plus time) in an underwater data set. With the aid of the rapid development of underwater acoustic communications, along with sophisticated AUV instrumentation, autonomy and control software, it is now feasible for an AUV to autonomously adapt its

Portions of this chapter are (C2010 IEEE. Reprinted, with permission, from S. Petillo, A. Balasuriya, and H. Schmidt, "Autonomous Adaptive Environmental Assessment and Feature Tracking via Autonomous Underwater Vehicles," Proceedings of OCEANS 2010 IEEE - Sydney. [3]

Portions of this chapter are (C2015 Springer. Reprinted, with permission, from H. Schmidt, M. R. Benjamin, S. Petillo, T. Schneider, and R. Lum, Ch. 10: "Nested Autonomy for Distributed Ocean Sensing," Springer Handbook of Ocean Engineering, in press. [51] 
motions to more intelligently and efficiently sample the environment through which it swims.

This autonomous and adaptive sampling with AUVs is achieved through a combination of Autonomous Adaptive Environmental Assessment (AAEA) and feature tracking methods and behaviors. AAEA is a process by which an AUV autonomously assesses the hydrographic environment it is swimming through in real time. This assessment is essentially the detection of hydrographic features of interest and leads naturally to the subsequent active/adaptive tracking of a selected feature. The detection-tracking feedback loop setup with AAEA currently aims to use solely an AUV's self-collected hydrographic data (e.g., temperature, conductivity, and/or pressure readings), along with a basic quantitative definition of an underwater feature of interest, to detect and track the feature. Feature tracking must be both autonomous in the sense that the AUV operator is not involved in guiding the vehicle outside of commanding it to "track feature X," and adaptive in the sense that, as a dynamic feature evolves over space and time, the AUV will recognize any changes and alter course accordingly to retain data coverage of the feature.

\subsection{Background \& Importance}

Two main fields of research are directly benefited by the implementation of AAEA on AUVs: engineering technology and oceanographic science. Currently, in the field of engineering, engineers who implement software on and deploy AUVs may not have the knowledge base of an oceanographer to determine where to fly the AUV to capture a desired hydrographic feature. Alternatively, oceanographers only have an educated guess (often based on models, theory, and past observations) as to where and when a feature is present in the water. The use of AAEA in conjunction with an autonomous control system on board an AUV gives the AUV a method of calculating the boundaries of the feature of interest and using that information to alter its course and more fully capture the feature's characteristics in its data.

\subsubsection{Science/Oceanography}

At-sea data collection is typically a very expensive and planning-intensive exercise for oceanographers, often limiting their ship time to a week or so every few years. They must conduct rigorous experiments during these times and hope that their predictions of when and where the features of interest may occur are sufficiently accurate. More accessible data sources frequently used by oceanographers include satellites, ship casts, floating profilers, buoys, and moored arrays. This restricts them to studying mostly what can be observed from these uncontrollable sources. The advantage to AUVs programmed with AAEA for feature tracking is that oceanographers using these vehicles have a higher likelihood of collecting a relevant data set with the 
information they need for furthering research, making their precious time at sea even more productive.

\subsubsection{Technology/Engineering}

Looking at the ocean from the perspective of an ocean engineer running, designing, or writing software for AUVs, there are visible limitations that the ocean imposes on the vehicles and operations. The vehicles can be run in a variety of locations and can be sent on complex missions, yet many engineers do not have a solid enough oceanographic background and may not understand how all of the puzzle pieces of the oceanographic environment interact to create a bigger picture. In this way, many engineers are unable to deploy AUVs on missions to sufficiently capture data sets characteristic of many environmental features (e.g., eddies, thermoclines, fronts, etc.).

Combining the knowledge of scientists with the tools of engineers is a significant benefit to the spread of knowledge and technology throughout both fields.

\subsection{Goals \& Motivation}

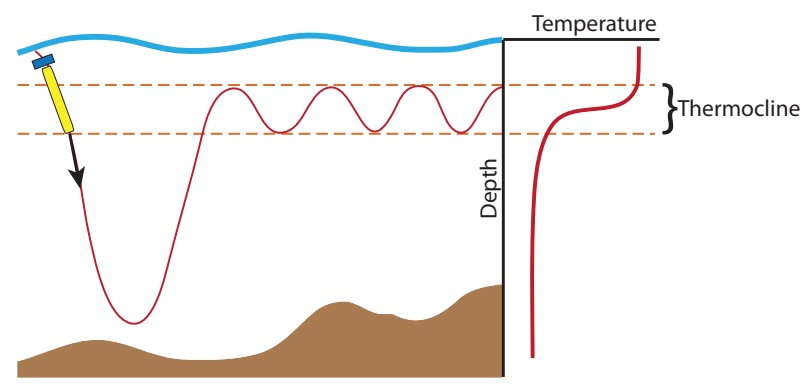

Figure 3-1: A conceptual sketch of thermocline tracking using an AUV, which collects and processes all necessary temperature data on board.

The first developments using AAEA for feature tracking have been applied to autonomously tracking the marine thermocline. The thermocline tracking procedure has been built up from concept (sketched in Fig. 31) to implementation, and finally tested in field experiments. This chapter outlines this procedure, following the guidelines for AAEA and feature tracking laid out in Section 3.5.

The thermocline was chosen as a simple, well-defined example of an oceanographic feature that is present in most large bodies of water (e.g., large lakes, seas, oceans). Hence, thermocline tracking is used as a proofof-concept for AAEA and feature tracking. Another reason to begin with thermocline tracking is that most AUVs are equipped with a CT (conductivity-temperature) or CTD (conductivity-temperature-depth) sensor, which collects the temperature (and depth) data necessary to detect the thermocline. 


\subsection{Literature}

At the time that this work was done and published (2008-2010) [3], a number of sources had mentioned the benefits of using AUVs for feature tracking and made some non-autonomous attempts [43-45], but there were very few pieces of literature that described attempts at any type of autonomous feature tracking with AUVs. One of the earliest adaptive feature sampling experiments using AUVs was described by Wang et al. for sampling the ocean acoustic environment in the northern Tyrrhenian Sea [40]. Subsequently, a variety of approaches to AAEA and feature tracking - particularly thermocline tracking (and later front and plume tracking, among others)—have been published.

Coincident with the publication of this work, Cruz and Matos presented a method of, and algorithms for, using an AUV for autonomous and adaptive thermocline tracking that is very similar to the method and algorithms presented here [52,53]. The primary differences are: 1) in the selection of thermocline upper and lower threshold values - though this work and theirs both base these values off of the vertical temperature gradient values evaluated across temperatures binned at discrete depths-and 2) the use of temperature averaging in this work over multiple vertical profiles, coupled with a timer that resets the temperature averages, versus their use of the temperature profile data from only the previous profile to determine the thermocline for the current profile. Both of these approaches are valid and reasonable, but they have not been evaluated against each other to determine if one or the other performs 'better' or more robustly.

Shortly thereafter, Zhang et al. published work on thermocline tracking based on tracking the peak vertical temperature gradient [54-57]. Similar to the work by Cruz and Matos using defined temperature gradient thresholds for the current profile that are a fraction of the peak gradient from the previous profile, Zhang et al. defined the thermocline peak area as a fixed depth range around the peak gradient depth and use the peak gradient depth of only the previous profile to define the depth range covered by the current profile. This method keeps the horizontal sampling of the thermocline constant, but is fragile to sudden increases or decreases in peak thermocline depth, which may occur in highly dynamic coastal and shelfbreak environments.

Although the thermocline tracking approach employed in this work did not originally have the ability to strictly follow the peak thermocline gradient, that capability was added shortly after the publication of the bulk of this work in 2010 and is presented here. It uses a temperature averaging method coupled with the Zhang et al. fixed depth range method around the thermocline peak mentioned above.

Details of the AAEA process and this thermocline tracking method and algorithms are described in the remainder of this chapter. 


\subsection{AAEA \& Feature Tracking: A Novel Approach}

In collecting data with AUVs, consider an AUV moving through the water in space and time and we want to know: where (or when) is feature X. Up until recently, AUVs have not had the ability to react to environmental variations in real time. Many AUVs are used for environmental monitoring, but the data is not processed on board the vehicle. Most data processing occurs post-mission on powerful, speedy computers in a lab, whereas processing on board AUVs must take a much more conservative, controlled approach. The motivation behind AAEA is to be able send the AUV on a mission to "track feature X", and the vehicle will make all proceeding decisions. To accomplish this, the AUV must use AAEA to process environmental data (from CTDs, ADCPs, fluorometers, etc.) on board the vehicle. This processing will determine where feature $\mathrm{X}$ occurs, allowing the AUV to autonomously react to its surroundings and track the feature.

Due to the restrictions of working with AUVs, which will be mentioned later, we are limited to determining the boundaries of feature X based on just the environmental information the AUV collects and processes on board. This must be done completely autonomously (with no human actively in the loop), allowing the AUV to make decisions of its own based on the environment it is swimming through.

Before the AUV begins AAEA, however, what feature to detect and track must determined, along with what measurable environmental state variables describe that feature.

\subsubsection{Oceanographic Features}

Almost every feature in the ocean environment is of interest to some scientist somewhere. Just a small subset of these features is given in Table 3.1. Many of these features are delineated by gradients of measurable environmental variables, e.g., temperature gradients define the vertical location of the thermocline.

\subsubsection{Defining a Feature Based on Data}

Before a feature in the ocean can be detected (by running a feature-detecting algorithm on a set of data), the feature must be defined. Hence, a robust quantitative definition must be developed for each feature and implemented in the form of an algorithm. This algorithm must also account for the temporal and spatial scales characteristic of an ocean feature, since many of these features are highly dynamic. Determining the physical spatial and temporal boundaries of a feature requires either research into the underlying processes that form the feature (not discussed in this thesis), or qualitative observations of the feature in plotted data, along with some general knowledge of the properties of the ocean environment. 
Table 3.1: Features, their measurable tracers, and associated instrumentation

\begin{tabular}{|l|l|l|}
\hline Features/Obesrvations & Measurable Tracers & Instruments \\
\hline \hline $\begin{array}{l}\text { Thermocline, halocline, pycno- } \\
\text { cline, sound speed }\end{array}$ & $\begin{array}{l}\text { Temperature, conductivity, pres- } \\
\text { sure }\end{array}$ & CTD [16] \\
\hline $\mathrm{O}_{2}$ concentration & $\begin{array}{l}\text { Partial pressure of } \mathrm{O}_{2} \text { in water } \\
\text { (via temperature \& conuctivity) }\end{array}$ & Dissolved Oxygen sensor [17] \\
\hline $\begin{array}{l}\text { Phytoplankton biomass \& } \mathrm{Cl} \\
\text { concentration }\end{array}$ & Chlorophyll-a fluorescence & Fluorometer [18] \\
\hline Light attenuation & $\begin{array}{l}\text { Photosynthetically Active Radi- } \\
\text { ation (PAR) of 400-700 nm } \\
\text { wavelength }\end{array}$ & PAR sensor [19] \\
\hline Currents & $\begin{array}{l}\text { Doppler (frequency) shift of } \\
\text { sound waves }\end{array}$ & ADCP [20] \\
\hline Fronts & $\begin{array}{l}\text { Temperature, conductivity, pres- } \\
\text { sure, Doppler shift }\end{array}$ & CTD, ADCP \\
\hline
\end{tabular}

\subsubsection{AAEA Process}

Once a feature-detecting algorithm has been created, it must be converted into a piece of code that can interface with the autonomy software of the AUV. This was addressed briefly in Section 2.3.1. The AUV will then have the ability to perform AAEA by processing its self-collected data using the algorithm code. This action determines the spatial and/or temporal boundaries of the feature in question.

\subsubsection{Tracking}

Knowing the boundaries of a feature, an interface is made with the on-board autonomy control to reposition the AUV. This repositioning, or path adjustment, is used to track a feature, i.e., collect a more complete data set describing the feature. Feature tracking is done by the AUV actively (yet autonomously) keeping itself within or around the feature's physical boundaries.

\subsection{Tracking the Marine Thermocline}

\subsubsection{Thermocline Definition}

By definition, the thermocline is "the region in a thermally stratified body of water which separates warmer surface water from cold deep water and in which temperature decreases rapidly with depth" [59]. Such a feature is shown in Fig. 3-2. From this (qualitative) definition, we can quantitatively define the thermocline 


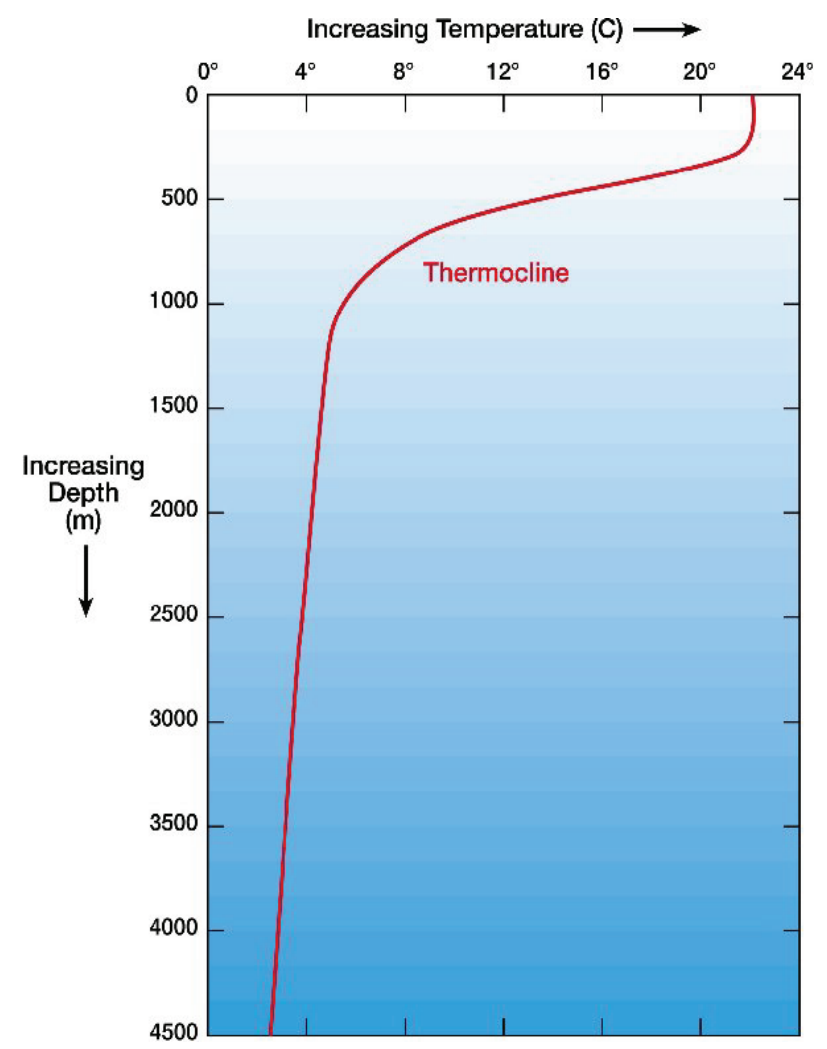

Figure 3-2: A typical mid-latitude oceanic Temperature vs. Depth profile. The thermocline region (that of most rapid decrease in temperature over depth) in this profile is apparent between about 300-1000 m depth. The strength of this thermal stratification is often highly variable, depending on location and over time. Image courtesy of Windows to the Universe [58].

as the depth range over which the vertical derivative of temperature, $\partial T / \partial z$, exceeds some threshold value, as depicted in Fig. 3-3.

\subsubsection{Algorithm: Thermocline Bounds and Maximum}

Constructing an algorithm from this definition is as follows. Assume an ideal temperature profile similar to that in Fig. 3-2 as an approximation to a profile obtained from CTD data (Fig. 3-3). Define variables $T$ for temperature in ${ }^{\circ} \mathrm{C}, z$ for depth in meters (positive up from the free surface), and $H$ for water depth in meters.

1. Calculate the slope of the temperature curve at each point in depth, $z^{\prime}$.

$$
\left.\frac{\partial T}{\partial z}\right|_{z^{\prime}}
$$

2. Average the vertical derivatives over the span of the water column. This yields the following threshold 


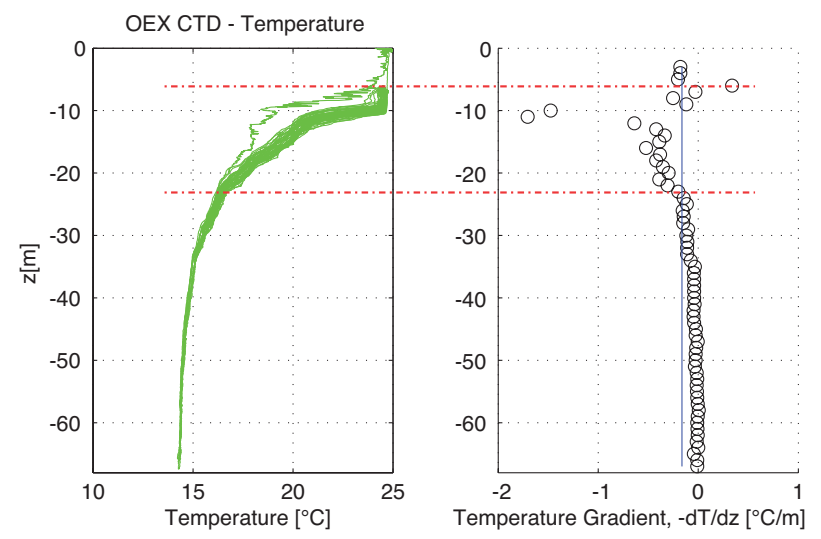

Figure 3-3: Temperature data through the water column (left) collected during GLINT '09 by the CTD aboard the NURC OEX AUV. On-board data processing was done to calculate the average temperature gradients at $1 \mathrm{~m}$ depth levels (circles, right) and through the entire water column (solid vertical line, right). The dashed lines indicate the upper and lower bounds of the detected thermocline region, calculated by pEnvtGrad (described in Section 3.6.3).

value.

$$
\left(\frac{\partial T}{\partial z}\right)_{\text {tot_avg }}=\left.\frac{1}{H} \int_{z^{\prime}=0}^{-H} \frac{\partial T}{\partial z}\right|_{z^{\prime}} d z^{\prime}
$$

3. Determine the upper and lower depth limits of the thermocline region.

$$
\begin{gathered}
\text { If }:\left|\frac{\partial T}{\partial z}\right|_{z^{\prime}}|\geq|\left(\frac{\partial T}{\partial z}\right)_{\text {tot_avg }} \mid \\
\text { Then : } z^{\prime} \text { within thermocline }\left(z_{\text {in_thermocline }}\right) \\
\text { upper_thermocline_depth } \equiv-\max \left(z_{\text {in_thermocline }}\right) \\
\text { lower_thermocline_depth } \equiv-\min \left(z_{\text {in_thermocline }}\right) \text {, }
\end{gathered}
$$

where depth is the distance, positive down, from the free surface.

4. Determine the depth of the maximum temperature change per unit depth.

$$
\text { maxgrad_thermocline_depth } \equiv-z_{\text {in_thermocline }} @\left|\frac{\partial T}{\partial z}\right|_{\max }
$$

An analogous determination can be done for the region of maximum sound speed variation over depth, which we will call the 'acousticline', or the halocline or pycnocline. 


\subsubsection{Algorithm Implementation on AUVs: pEnvtGrad}

The algorithm described above is implemented on an AUV as a piece of code called $p$ EnvtGrad, which stands for 'process: Environmental Gradient.' This code calculates the vertical temperature and sound speed gradients and quantitatively defines and detects both the thermocline and acousticline. [Note: It is up to the AUV operator to determine which of these features to track. Here we will continue with tracking the thermocline, since sound speed variations are dominated by temperature variations in coastal and surface ocean waters.] This process then interfaces with the MOOS-IvP autonomy to guide the AUV in a vertical yo-yo pattern (see Fig. 3-1) between the upper and lower thermocline depth limits, continuously adapting the limits to the variations in thermocline range over space and time. A more detailed description of the steps taken within pEnvtGrad are given below.

1. Initial yo-yo:

At the start of the mission the AUV dives from the surface to as deep as is allowable to get as complete a data set as possible over the water column.

2. Create depth "bins":

The water temperature data is split up into vertical depth levels, or "bins." This defines the discrete depth levels to work with.

3. Average $T$ in each bin:

The temperature values within each depth bin are averaged to eliminate sub-scale variations in temperature. It is memory-consuming and not useful to determine thermocline bounds on the sub-1-meter scale when the bounds of the thermocline itself are not absolutely defined. A brief scales analysis of depth bin size and thermocline depth range is given in Table 3.2.

4. Vertical derivative, $\Delta T / \Delta z$, over adjacent bins:

The discrete vertical derivative over each pair of adjacent bins is computed.

$$
\left.\left.\frac{\partial T}{\partial z}\right|_{z^{\prime}} \approx \frac{\Delta T}{\Delta z}\right|_{b i n_{-} i, b i n_{-}(i+1)}=\frac{T_{i+1}-T_{i}}{z_{i+1}-z_{i}}
$$

5. Threshold: 
Average $\Delta T / \Delta z$ over the sampled water column.

$$
\left(\frac{\Delta T}{\Delta z}\right)_{\text {tot_avg }}=\left.\frac{1}{n-1} \sum_{i=1}^{n-1} \frac{\Delta T}{\Delta z}\right|_{b i n \_i, b i n \_(i+1)},
$$

where $n$ is the total number of depth bins in the water column.

6. Determine the thermocline depth range, where $|\Delta T / \Delta z|$ is greater than the threshold value:

$$
\begin{aligned}
& \text { If }:\left|\frac{\Delta T}{\Delta z}\right|_{z^{\prime}}|\geq|\left(\frac{\Delta T}{\Delta z}\right)_{\text {tot_avg }} \mid \\
& \text { Then }: z^{\prime} \text { within thermocline }\left(z_{\text {in_thermocline }}\right),
\end{aligned}
$$

where $z^{\prime}$ is the depth bordering between bin_i and bin_ $(i+1)$. Thus,

$$
\begin{aligned}
& \text { upper_thermocline_depth } \equiv-\max \left(z_{\text {in_thermocline }}\right) \\
& \text { lower_thermocline_depth } \equiv-\min \left(z_{\text {in_thermocline }}\right) .
\end{aligned}
$$

7. Determine the depth where $|\Delta T / \Delta z|$ is maximized:

$$
\text { maxgrad_thermocline_depth } \equiv-z_{\text {in_thermocline }} @\left|\frac{\Delta T}{\Delta z}\right|_{\max }
$$

8. Track the thermocline:

Adjust yo-yo depth limits continuously (and autonomously) by keeping a running average of the temperature data collected for each bin. In this way, the thermocline tracking process is adaptive to its dynamic environment.

\section{Periodic reset:}

After a fixed amount of time, $t_{R}$, reset the gradient determination process by 'forgetting' all previous data, and start over from the initial yo-yo. Ideally $t_{R}$ is not longer than half the length of the characteristic time scale, $t_{0}$, over which there is a significant change in the feature. That is,

$$
t_{R} \leq \frac{t_{0}}{2}
$$


Table 3.2: Scaling of depth bins with water depth

\begin{tabular}{|l|l|l|l|}
\hline & Water Depth & Thermocline Range & Depth Bin Range \\
\hline \hline $\begin{array}{l}\text { Shallow water/coastal } \\
\text { system }\end{array}$ & $\mathrm{O}(100 \mathrm{~m})$ & $\mathrm{O}(10 \mathrm{~m})$ & $\sim 1 \mathrm{~m}$ \\
\hline Open ocean & $\mathrm{O}(1000 \mathrm{~m})$ & $\mathrm{O}(100 \mathrm{~m})$ & $\sim 10 \mathrm{~m}^{\mathrm{a}}$ \\
\hline
\end{tabular}

${ }^{a}$ Here it is natural to scale all values up by one order of magnitude from shallow water to the open ocean, however the open ocean may also have a second, transient near-surface thermocline $(\mathrm{O}(10 \mathrm{~m})$ range $)$ that would require a depth bin range of $\sim 1 \mathrm{~m}$ to adequately capture.

Essentially, this is a reset at the Nyquist frequency of the feature's variations. In tracking a coastal thermocline over the course of a day, there may be significant changes in thermocline depth as the surface warms from the sun and begins to mix due to winds in the morning, and then cools again in the evening. In such cases, we may see variations in thermocline depth over the course of a couple hours $\left(t_{R} \approx 0.5-1.0 \mathrm{hr}\right)$, whereas calmer, cloudier days may see variations on the scale of 3-6 hours $\left(t_{R} \approx 1.5-3.0 \mathrm{hr}\right)$ or longer, depending on location and season.

\subsubsection{Virtual Experiments \& Testing}

The final steps of the implementation process involve testing pEnvtGrad in virtual experiments before an AUV can be deployed on a thermocline tracking mission. Using a MOOS-IvP interface to a dynamic ocean model (in the MSEAS NetCDF format $[60,61]$ ), we can simulate an AUV flying through a dynamic ocean and autonomously tracking the thermocline as if it were actually in the water. The results of this testing are plotted in Fig. 3-4. The MOOS-IvP simulation (virtual experiment) interface is nearly identical to the runtime interface (used during an actual mission), making the transition from simulation to runtime virtually seamless.

\subsection{Field Experiments \& Results}

With the implementation and testing completed, field experiments to track the thermocline (and acousticline) could be conducted. Adaptive thermocline and acousticline tracking were demonstrated during the GLINT '09, Champlain '09, and GLINT '10 field trials, which are described below. The GLINT '10 experiment in particular used adaptive thermocline tracking missions in the broader context of collecting a synoptic multiAUV data set displaying evidence of internal waves, which is discussed in more detail in Chapter 4. Here it is 

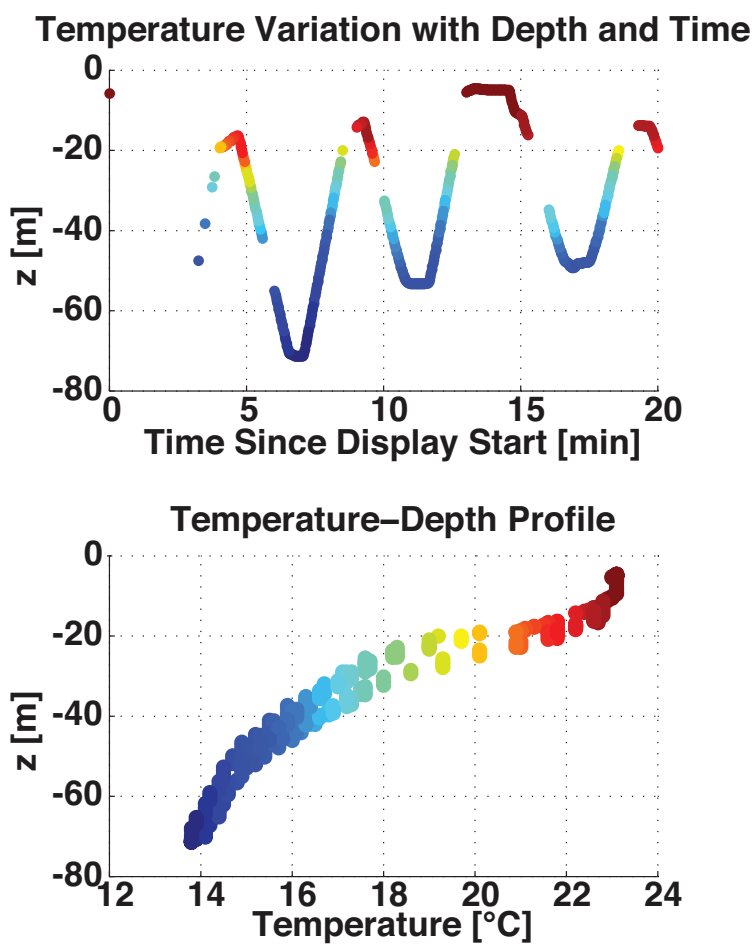

Figure 3-4: These plotted mission data are from a virtual experiment run using $p E n v t G r a d$ to track the surface thermocline in the Middle Atlantic Bight region. This region was modeled by the MIT MSEAS group [60] in early 2008 from data collected in that region during the Shallow Water '06 experiment in late August, 2006 [62]. These plots show (top) the AUV depth over time and (bottom) the temperature-depth profile. Coloring corresponds to the temperature indicated by the bottom plot. In the upper plot, the initial (deep) yo-yo is seen, followed by a few shallow yo-yos between the depths of 12 and $52 \mathrm{~m}$, indicative of tracking the thermocline when compared to the thermocline depth range of the lower plot (about 10-50 m).

useful to first become familiar with the 'topside' setup used by MIT on board the ship to deploy and monitor the AUVs underwater.

\subsubsection{MIT Topside Setup}

On board a research vessel, the lab is set up with laptops from which the AUVs are commanded. The ship has a GPS link for positioning, which is decoded on the topside laptop, allowing a determination of where the ship is relative to the AUV. The AUV itself has acomms with the ship (and topside computer) via ship- and AUV-mounted acoustic modems, so status messages, commands, and minimal amounts of data can be sent between the two platforms. The command and control station setup on the topside computer includes Goby Liaison (a web-browser-based GUI that functions as a rapidly reconfigurable mission commander) that allows the AUV to be commanded to, e.g., track the thermocline for $1 \mathrm{~km}$ heading $45^{\circ}$, and it will deploy itself on 


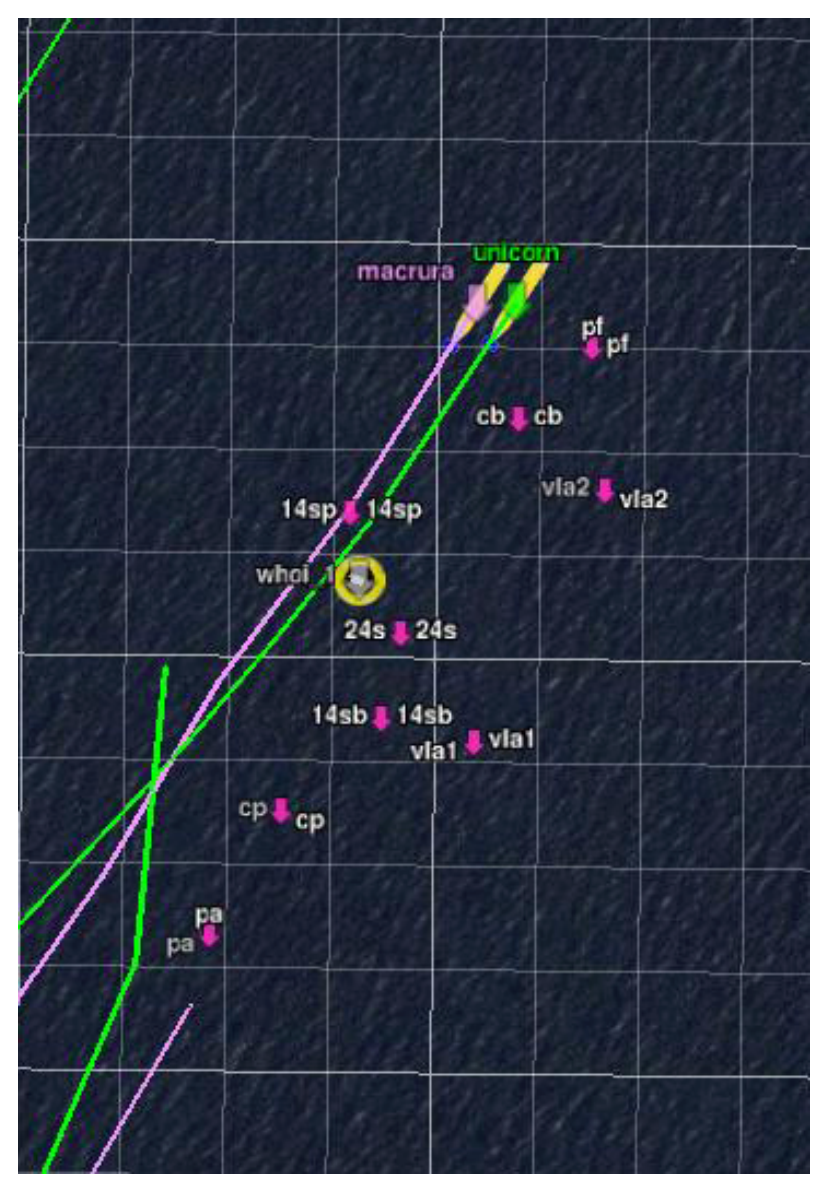

Figure 3-5: The Google Earth interface for Ocean Vehicles (GEOV) [63] real-time topside situation display in Google Earth [64] showing two AUVs swimming in sync (trailed by purple and green lines) past an acoustic communications buoy (yellow circle) and a field of objects on the sea floor (pink arrows).

that mission. The topside situation display includes a display of the incoming CTD data in near real time (similar to that shown in Fig. 3-4), as well as the Google Earth interface for Ocean Vehicles (GEOV) [63]. GEOV, as shown in Fig. 3-5, is a very useful real-time display of the positions of the research vessel, AUV(s), and their recent paths, in Google Earth [64]. It is an integral piece in the planning and monitoring of AUV missions.

\subsubsection{GLINT '09-Acousticline Tracking}

The GLINT '09 experiment took place in the Tyrrhenian Sea near Porto Santo Stefano, Italy. Adaptive feature tracking missions were run 13-14 July, 2009, with the coordinated efforts of MIT and the NATO Undersea Research Centre (NURC, based in La Spezia, Italy). The NURC OEX AUV (shown in Fig. 3-6) running the MOOS-IvP autonomy system was deployed from the R/V Alliance for adaptive feature tracking missions. 


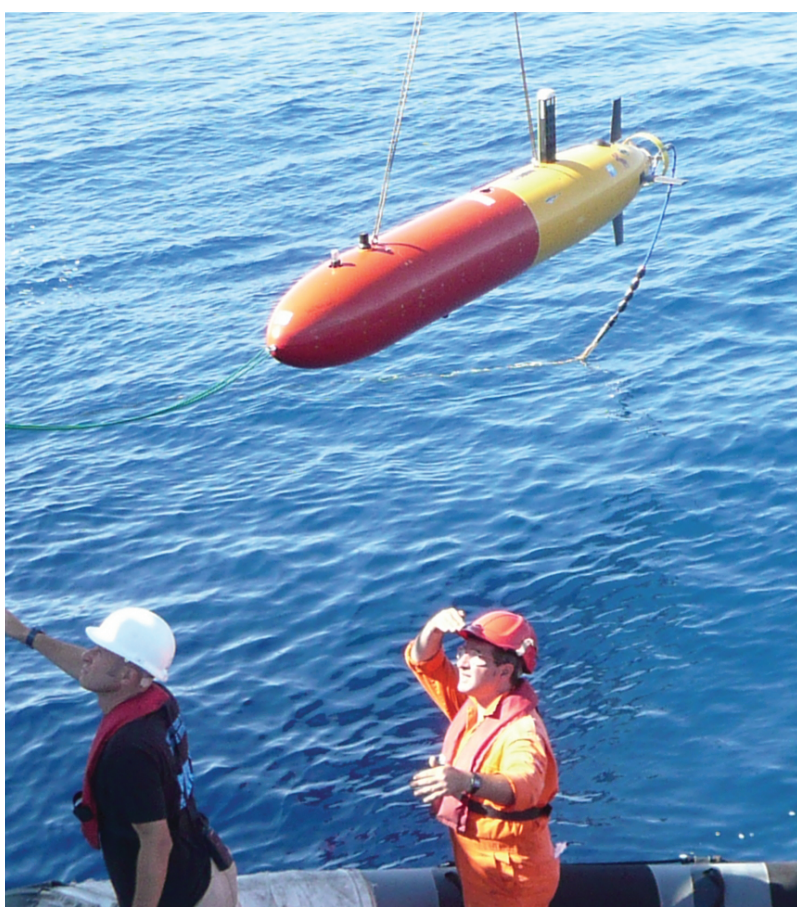

Figure 3-6: The NURC OEX AUV used during GLINT '09. This AUV communicates with the ship via acomms (underwater). It also carries a GPS for positioning.

During this cruise, pEnvtGrad underwent development and testing in virtual experiments before its first sea trial. The mission during these days was to track the acousticline. The AUV was deployed into a northsouth racetrack pattern of $1000 \mathrm{~m} \times 200 \mathrm{~m}$ and performed an adaptive-depth yo-yo pattern based on the acousticline depth determined by $p$ EnvtGrad.

\subsubsection{GLINT '09 Results}

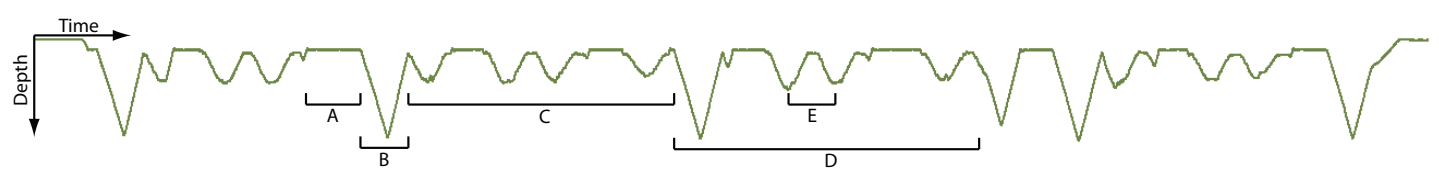

Figure 3-7: Depth history of the OEX AUV during an adaptive acousticline tracking mission. (A) is the default shallow turning and transiting depth $(7 \mathrm{~m})$. (B) is the initial yoyo $(7-70 \mathrm{~m})$ performed by the AUV to ensure sampling of the entire water column down to the vehicle's maximum dive depth. (C) is the adapted yo-yo tracking the acousticline between 9 and $28 \mathrm{~m}$ depth. (D) is a $30 \mathrm{~min}$ tracking period after which the AUV re-initializes the yo-yo through the full water column to account for acousticline depth variation over space and time. (E) is the $400 \mathrm{~m}$ period (length) of a single yo-yo.

Fig. 3-7 shows the actual depth of the OEX AUV over the course of approximately 2 hours. The initial yo-yo is visible as the deep dive from 7 to $70 \mathrm{~m}$ and back, and then the OEX began tracking the acousticline 
between 9 and $28 \mathrm{~m}$ depth (smaller amplitude undulations). The depth bins were chosen to be $1 \mathrm{~m}$ deep (due to a water depth of about $105 \mathrm{~m}$ ) and the periodic reset was set to 30 minutes.
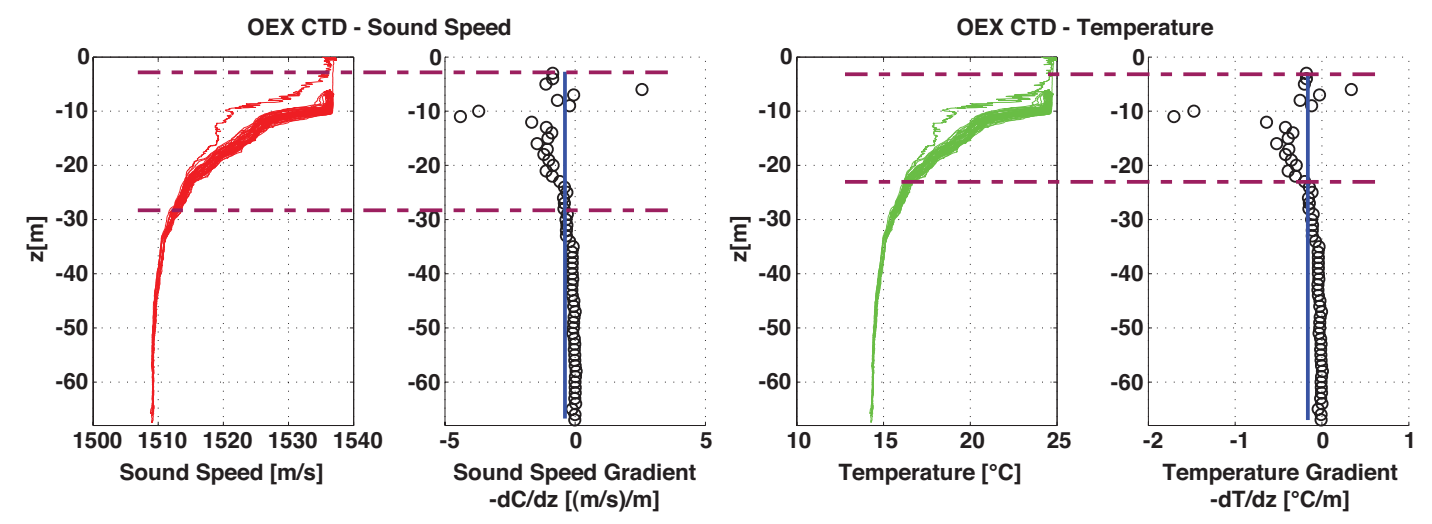

Figure 3-8: The leftmost plot of each pair gives the sound speed-depth (left) and temperature-depth (right) profile, respectively, over the entire mission (multiple dives). The rightmost plot of each pair shows the vertical sound speed (left) and temperature (right) gradients averaged over 1-meter depth bins. The solid vertical blue lines (on the gradient plots) represent the threshold values (average gradient over all sampled depths). A gradient greater in magnitude than the threshold magnitude is determined to be within the depth range of the acousticline or thermocline, respectively. The acousticline and thermocline regions are bounded by the dashed lines shown.

Post-processing of the sound speed and temperature data from the entire 2+ hour mission (see Fig. 3-8) shows the similarities in the shape of the sound speed and temperature profiles in this region. This is due to the fact that sounds speed is dominated by temperature in shallow waters such as these and in the upper layer of the ocean, and by pressure deep in the ocean. The formula used here to calculate sound speed is the MacKenzie Sound Speed Equation (1981) [65]. The calculated average acousticline depth range was 3-28 $\mathrm{m}$ with a threshold total average gradient $\left((\Delta c / \Delta z)_{\text {tot_avg }}\right.$, where $c$ is the sound speed through the water in $\mathrm{m} / \mathrm{s})$ of $0.427(\mathrm{~m} / \mathrm{s}) / \mathrm{m}$, while the calculated average thermocline depth range was 3-23 $\mathrm{m}$ with a threshold total average gradient $\left((\Delta T / \Delta z)_{\text {tot_avg }}\right)$ of $0.162^{\circ} \mathrm{C} / \mathrm{m}$.

The primary difference between the 9-28 m acousticline range tracked by the AUV and the 3-28 m range calculated in post-processing is that the post-processing range also included some near-surface sound speed data collected during deployment and surfacing for getting GPS position locks. On board, the acousticline determination is limited to the data collected within the initial yo-yo range (7-70 $\mathrm{m}$ in this case), slightly increasing the threshold value and setting a deeper upper acousticline boundary (at $9 \mathrm{~m}$ ) than that calculated in post-processing. 


\subsubsection{Champlain '09—Thermocline Tracking}

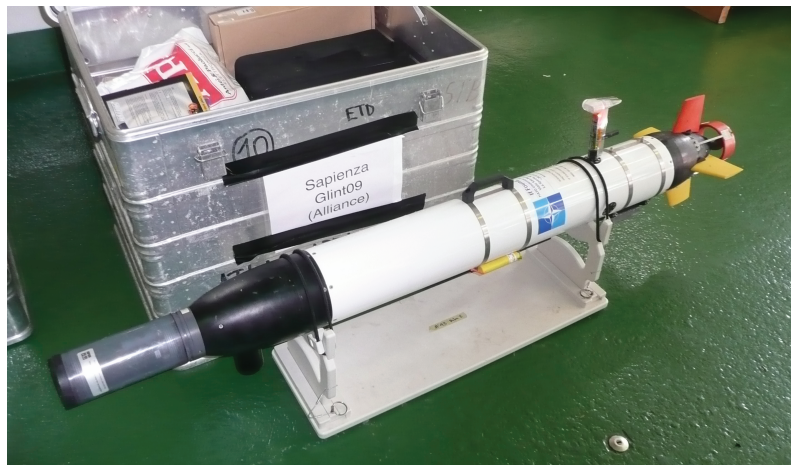

Figure 3-9: The NUWC Hammerhead Iver AUV used during Champlain '09. This AUV carries a complete environmental package in its nose and communicates with the ship via RF (on the surface) and acomms (underwater). It also carries a GPS and Doppler Velocity Logger (DVL) for positioning.

The Champlain '09 experiment took place in Lake Champlain, VT, USA (a freshwater lake). Adaptive feature tracking missions were run 3-5 October, 2009, with the coordinated efforts of MIT and the Naval Undersea Warfare Center (NUWC, based in Newport, RI, USA). The NUWC Iver AUV (shown in Fig. 3-9) running the MOOS-IvP autonomy system was deployed from a small motor boat for adaptive feature tracking missions.

During this experiment, pEnvtGrad underwent further testing and its second sea trial. The mission during these days was to track the thermocline of the lake. The AUV was deployed into a northwest-southeast straight line pattern $1 \mathrm{~km}$ long and performed an adaptive-depth yo-yo pattern based on the thermocline depth determined by $p$ EnvtGrad.

\subsubsection{Champlain '09 Results}

Fig. 3-10 (left, colored data points) shows the actual depth of the Iver AUV over the course of approximately 2 hours, about 1.5 hours of which it is deployed on the thermocline tracking mission. The initial yo-yo is visible as the first dive from 3 to $30 \mathrm{~m}$, and then the Iver begins tracking the thermocline between about 14 and 29 $\mathrm{m}$ depth (smaller amplitude undulations). The depth bins were chosen to be $1 \mathrm{~m}$ deep (due to a water depth on the order of $100 \mathrm{~m}$ ) and the periodic reset was set to 30 minutes. This plot also displays the thermocline depth bounds (left, green lines) calculated by pEnvtGrad, which, when plotted with the data of the AUV's actual depth over time (left, colored data points) shows that the AUV is able to actively and autonomously track the thermocline, adjusting to a change in thermocline depth of even a meter over a couple dives.

Post-processing of the temperature data from the entire mission (see Fig. 3-11) results in an average 


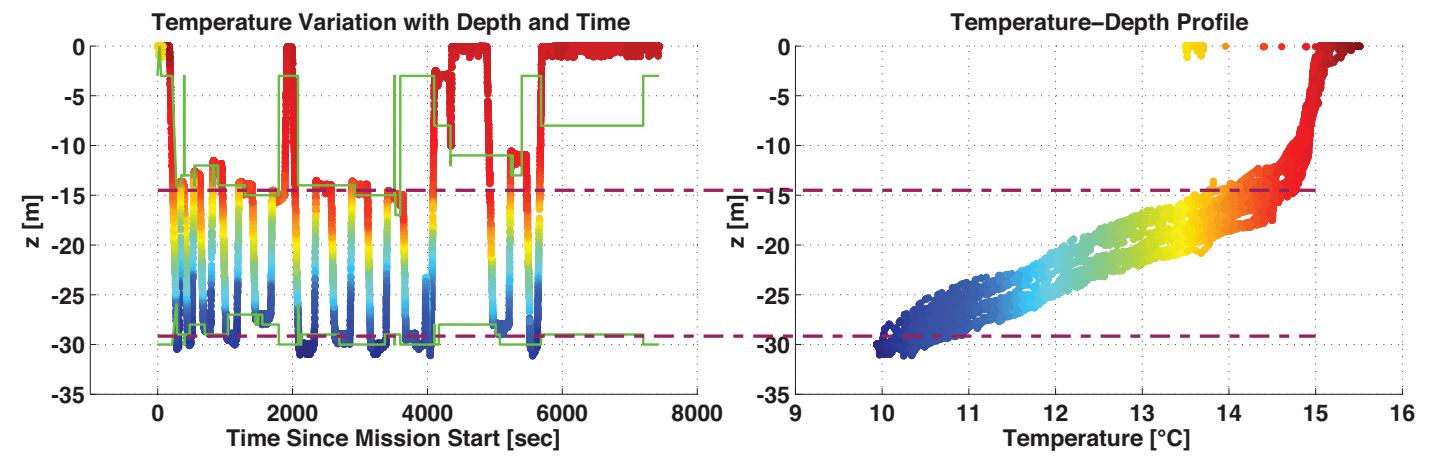

Figure 3-10: These data were taken from the real-time topside CTD display showing temperature variations over depth and time. The colors of the data on the left plot correspond to the temperature color coded by the right plot. The squared-off green lines across the plot on the left give the exact values of the thermocline boundaries as determined by $p E n v t G r a d$ throughout the mission. The dashed red lines approximate (by inspection) the average thermocline bounds as determined by $p$ EnvtGrad.

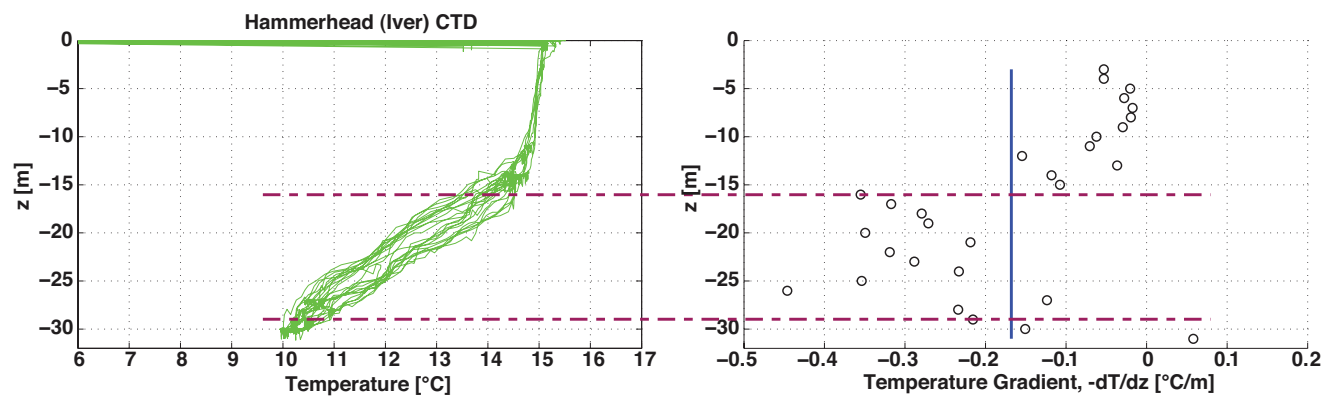

Figure 3-11: The plot on the left gives the temperature-depth profile over the entire mission (multiple dives). The plot on the right shows the vertical temperature gradients averaged over 1-meter depth bins. The solid vertical blue line (right) represents the threshold value (average gradient over all sampled depths). A gradient greater in magnitude than this average value's magnitude is determined to be within the depth range of the thermocline, the region bounded by the dashed lines.

thermocline depth range of about 16-29 m with a threshold total average gradient $\left((\Delta T / \Delta z)_{\text {tot_avg }}\right)$ of $0.168{ }^{\circ} \mathrm{C} / \mathrm{m}$. This average thermocline range is very close to that determined by inspection of the AUV's actions and calculations in Fig. 3-10 (left).

Relating back to the generally qualitative definition of a thermocline and its algorithm developed earlier in this chapter, it is essential to keep in mind that all of the calculated thermocline (and acousticline) bounds are relative to the threshold value, which is relative to the depth of the water column that can be sampled by an AUV. In some cases such as this, the AUV could not risk diving much deeper than $35 \mathrm{~m}$ (due to a very muddy lake bottom) and only captured part of the thermocline. However, this also shows that $p$ EnvtGrad as a thermocline detecting algorithm is robust enough to still detect the majority of the thermocline range even without full water column coverage. 


\subsubsection{GLINT '10-Thermocline Tracking for Internal Wave Detection}

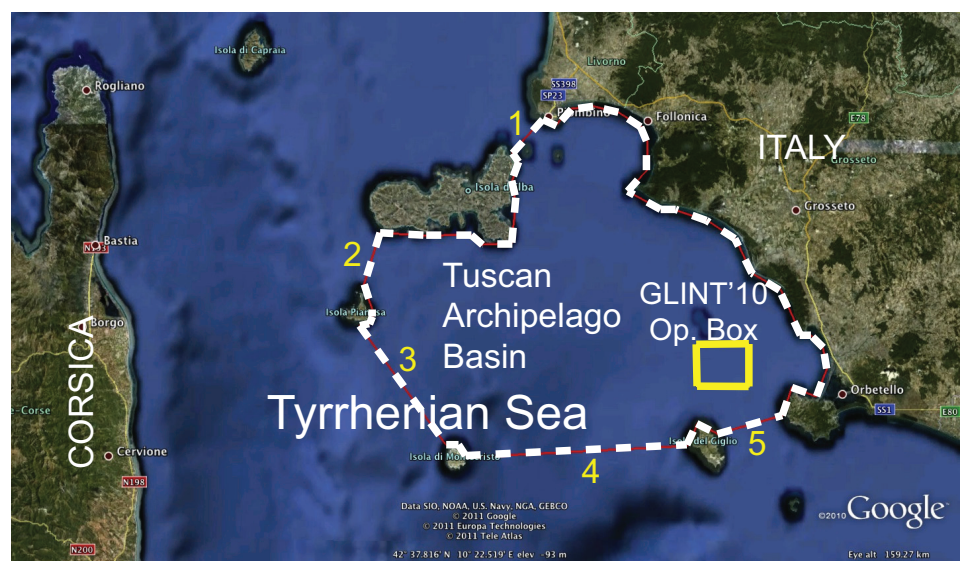

Figure 3-12: The region of the Tyrrhenian Sea bounded by the western coast of Italy and the islands of the Tuscan Archipelago. The Tuscan Archipelago basin is outlined by the dashed line. The GLINT '10 AUV operation region is delineated by the box. The numbering shows the five inlets of the basin.

The GLINT '10 Internal Wave Detection Experiment took place on 13 August, 2010, as a collaborative effort between groups from MIT and NURC. The area of study was the northern region of the Tyrrhenian Sea bounded by the Tuscan Archipelago and the west coast of Italy. The AUVs were operated in the southeastern area of this Tuscan Archipelago basin, which is delineated by the yellow 'GLINT '10 Op. Box' in Fig. 3-12. This experiment aimed to use adaptive autonomous sampling and multiple AUVs to detect the presence of internal waves (or lack thereof) in this region of the Tyrrhenian Sea.

Internal waves are supported along density interfaces, such as the pycnocline, so we decided to use the AUVs to monitor the temperature in this environment. That is because the thermocline corresponds in depth with the pycnocline in a coastal ( $~ 100 \mathrm{~m}$ depth) isohaline environment such as the Tuscan Archipelago basin. In order to collect fully synoptic data sets most likely to exhibit the presence of any passing internal waves in this dynamic environment within the few hours of AUV deployment time available, a novel multi-AUV, 3D approach was needed for the AUV sampling strategy. With two 21" diameter AUVs available, we were able to use autonomous following and adaptive thermocline sampling techniques on board the vehicles while they communicated and autonomously collaborated with each other during the internal wave detection missions to get the most synoptic data sets possible.

Both AUVs ran the MOOS and IvP Helm autonomy software and were able to communicate real-time data and status updates between each other and the ship-board operators using acoustic communication systems. These features allowed the AUVs to autonomously coordinate their motions in the horizontal plane with a track-and-trail behavior, as seen in the topside display in Fig. 3-13. In the vertical axis, each AUV was 


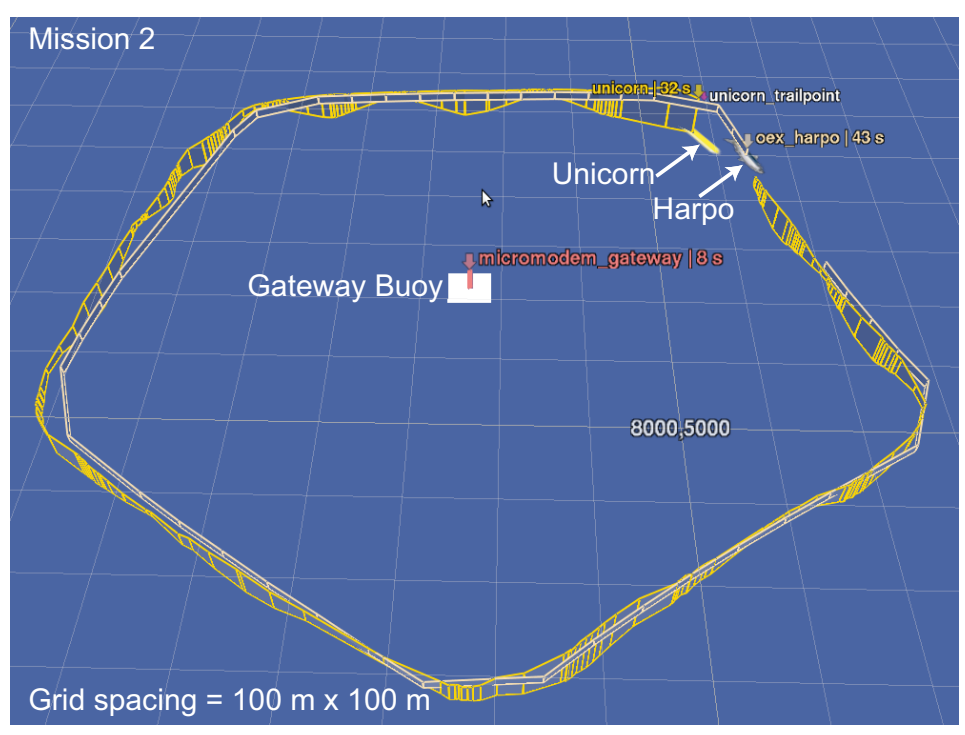

Figure 3-13: Mission 2 loiter pattern around the gateway buoy, as seen from above, at an angle to the horizontal. Harpo performs a horizontal loiter pattern at constant depth $(12 \mathrm{~m})$ just below the thermocline. Unicorn trails directly behind Harpo while performing an adaptive yo-yo pattern through the thermocline depth range. Vertical bars along the loiter indicate the AUVs' depths (yellow is Unicorn's track, white is Harpo's track), and their current positions are shown by the arrows. Best viewed in color.

given a different task. The Unicorn AUV preformed the adaptive thermocline tracking behavior, adapting its depth to the temperature changes in the environment while trailing behind the Harpo AUV. The Harpo AUV was following a pentagonal loiter pattern in the horizontal plane and maintained a constant depth at $12 \mathrm{~m}$, just below the thermocline depth (the depth at which $|\Delta T / \Delta z|$ is greatest—about $11 \mathrm{~m}$ ), since the sudden change in temperature at the thermocline was inhibiting acoustic communication when Harpo originally adapted its depth to match the thermocline depth. In addition to the two AUVs, a vertical thermistor chain was deployed during the Internal Wave Detection Experiment to capture any lower-frequency temperature oscillations and serve as a ground truth for the AUV data.

\subsubsection{GLINT'10 Results}

The results from this experiment strongly suggest that the propagation of internal waves is present in the Tuscan Archipelago basin. Thus, when the thermocline is well defined in the Tuscan Archipelago basin (primarily during the summer), it is likely that internal waves are detectable along the thermocline throughout the rest of the basin beyond the AUV operation region. This is a rather important oceanographic finding for those who perform acoustic and other oceanographic experiments in this region, as there is no literature todate suggesting the presence or absence of internal waves in the Tuscan Archipelago basin. For more detailed 
results and analysis from the Internal Wave Detection Experiment, see Chapter 4 and [66].

\subsection{Conclusion}

By implementing AAEA on board AUVs with acomms ability and MOOS-IvP autonomy, a means of autonomously detecting and actively tracking oceanographic features in situ, in near real time, on board an AUV has been developed. The thermocline tracking example is a successful proof-of-concept for autonomous detection and tracking of hydrographic gradients using AAEA. This is especially important because most hydrographic features are characterized or delineated by gradients or concentrations of environmental tracer(s).

In this chapter the step-by-step process of developing AAEA and feature tracking from concepts through testing in field experiments has been described. The thermocline and acousticline detection algorithm implemented in the pEnvtGrad code was tested successfully in conjunction with MOOS an IvP Helm autonomy on AUVs during the GLINT '09, Champlain '09, and GLINT '10 field experiments. This demonstrates that pEnvtGrad is robust enough to handle thermocline/acousticline tracking in both freshwater and saline environments and is seamlessly adaptable to use on very different AUVs running the same (MOOS and IvP Helm) autonomy system.

With the use of adaptive sampling, autonomy, and acoustic communication on the AUVs, the human can be taken out of the loop in the sampling process while improving the data collected. This approach significantly reduces the ship time required for collecting a specific data set by sampling only the areas of interest to the scientists' research instead of doing large pre-planned surveys in hopes of collecting the desired data somewhere within the survey area. Since many oceanographic studies still use manual shipside deployment of instruments at discrete locations to collect data, the integration into the oceanographic community of AUVs with the ability to perform environmentally adaptive sampling using AAEA and feature tracking will be an important step for the future of oceanographic research.

The three experiments described in Section 3.7 demonstrate the significant impact of using AAEA and feature tracking on AUVs to improve the efficiency and synopticity of oceanographic data collection. The novel feature tracking methods presented here include environmentally adaptive, fully autonomous tracking of the thermocline and acousticline depths as well as using multiple autonomously collaborating AUVs to increase 3D data coverage and synopticity. The latter of these methods is explored more fully in Chapter 4.

The natural continuation of this work is to further expand this process across multiple AUVs swimming in an area and interacting (via acomms) to paint a clearer picture of the ocean environment on small and large scales. Essentially, this will result in better data coverage over time and space. Tracking more complex 
oceanographic features over two or more dimensions (rather than just one, i.e., depth), such as eddies, oceanographic fronts, and bathymetry contours, is a useful development that requires multi-AUV data exchange and feature determination techniques to be adapted to capture the motion of highly dynamic (and larger-scale) features autonomously. Once multiple AUVs identify the bounds of a single feature, the challenge is then to coordinate their paths such that they will (collectively) track the feature, continuously and autonomously adapting to the feature's motions. This is addressed in Chapters 5 and 6, where results are presented from front tracking autonomy behaviors that have been developed and tested for this research, and concepts are presented for an extension to plume tracking, respectively. 


\section{Chapter 4}

\section{Internal Wave Detection Experiment}

\subsection{Introduction}

Advances in the fields of autonomy software and environmental sampling techniques for autonomous underwater vehicles (AUVs) have recently allowed for the merging of oceanographic data collection with the testing of emerging marine technology. The Massachusetts Institute of Technology (MIT) Laboratory for Autonomous Marine Sensing Systems (LAMSS) group conducted an Internal Wave Detection Experiment in August 2010 with these advances in mind. The method of AAEA was applied with the use of the thermocline tracking algorithms described in Chapter 3 and a knowledge of the general characteristics of internal waves and where they propagate in the water column. The goal was to have multiple AUVs collaborate autonomously through on-board autonomy software and real-time underwater acoustic communication to monitor for the presence of internal waves by adapting to changes in the environment (specifically the temperature variations near the thermocline/pycnocline depth, where internal waves are most likely to propagate).

\subsection{Goals}

The GLINT '10 Internal Wave Detection Experiment aimed to use multiple AUVs to detect and characterize the presence of internal waves (or lack thereof) in the region of the Tyrrhenian Sea bounded by the western coast of Italy and the islands of the Tuscan Archipelago (see Fig. 3-12).

Portions of this chapter are (C2014 IEEE. Reprinted, with permission, from S. Petillo and H. Schmidt, "Exploiting Adaptive and Collaborative AUV Autonomy for Detection and Characterization of Internal Waves," IEEE Journal of Oceanic Engineering. [66] 
The primary constraints were the necessity to have multiple AUVs collaborate their positions autonomously to execute the experiment and to make use of the ability to adapt AUV position to temperature changes in the environment, harnessing the AAEA method developed in Chapter 3. In these coastal Mediterranean waters ( $110 \mathrm{~m}$ depth) with relatively constant salinity over depth, the water temperature dominates the density calculation in the equation of state for seawater [67]. This allows us to detect the presence of internal waves directly from the CT sensor's temperature measurements instead of needing to calculate density for each point in space. If successful, this experiment would be the first to use fully autonomously-collaborating AUVs that autonomously adapt their motion to changes in the environment, thus efficiently capturing a synoptic data set that may contain internal wave signatures.

There was a significant effort put forth to successfully demonstrate the use of AUVs of different types, from different research groups, communicating and collaborating autonomously through MOOS and IvP Helm autonomy software and acoustically communicating using a predefined polling scheme that is set using the pAcommsHandler underwater networking application—a MOOS interface (further described in Section 4.5.3) to the Goby-Acomms libraries.

This chapter will cover the motivation for the Internal Wave Detection Experiment on August 13, 2010, during the GLINT '10 field trials in the Tyrrhenian Sea west of Italy, and compare its goals with similar experiments from other literature. This is followed by details of the experimental setup and implementation from GLINT '10, including a discussion of the required instrumentation, communication, and autonomy systems. The resulting data sets from the AUV missions are then analyzed and compared with wave and buoyancy theory $[68,69]$ to determine the possible sources for dominant internal wave frequencies in the data. Finally, directions of future work are discussed and conclusions are drawn.

\subsection{Motivation}

Bodies of water in nature tend to be stably stratified with fluid density increasing with depth. This density variation is dependent upon water temperature, salinity, and pressure through the equation of state for seawater [67]. When an abrupt change in water density occurs over a short depth range, often referred to as a pycnocline, the boundary between the two layers of different-density seawater may support internal waves. That is, the strongly stable stratification of the density layers at the pycnocline will react with a restoring force when perturbed by water from above being forced downward or water from below being forced upward, creating an internal wave that will propagate away from its source along an isopycnal within the pycnocline [68]. Perturbations from internal waves can occur from a variety of sources, such as currents flowing rapidly past 
a narrow mouth to a basin, or waves produced by flow over underwater mountains or canyons near a shelfbreak. Internal waves frequently occur in regions where a strong thermocline is present and salinity can be considered constant (the pycnocline depth will then be coincident with that of the thermocline in shallow water).

Internal waves have a strong effect on acoustic propagation in any body of water, since sound waves travel as pressure waves that can be refracted in different directions as the acoustic impedance of the water changes. Acoustic propagation is used in oceanography for a variety of tomographic experiments and for underwater communication and data transfer when collecting data with autonomous underwater vehicles (AUVs). If the acoustic channel is disturbed by an undetected internal wave, errors in tomographic measurements and unpredicted loss of communication or data transfer to and from AUVs can result.

More specific to the field of oceanography, internal waves of large anplitude and long wavelength relative to water depth can transport a significant amount of energy from one location to another, and those that grow large enough to break along an isopycnal result in mixing between density layers and potential transport of biomass. Internal waves that propagate long distances shed light on the strength of currents and topography interacting both far from coastlines and right off the continental shelf.

In defense applications, detecting the presence of internal waves in an area may reveal the location of a submerged submarine, which generates internal waves through its motion underwater. Internal waves also interact with the acoustic propagation environment during target (mine) detection and ASW (anti-submarine warfare) operations, causing unpredicted bending in the path of propagating sound waves and thereby (possibly) revealing or concealing potential targets by insonifying an unpredicted area.

\subsection{Literature}

Until recently, most field studies of internal waves have been carried out using synthetic aperture radar (SAR) [70-72], acoustic tomography and altimetry [73], current meters on moorings [74], CTD (conductivitytemperature-depth) and XBT (expendable bathythermograph) casts [74], and satellite observations (photographs in varying light spectra) [75]. The goal of our experiment, however, was to use AUVs to determine if internal waves were present in our deployment region (more specifics are found in Sections 4.2 and 4.5). The specific AUVs for this experiment are actively propelled and able to sense and adapt to their local environment using on-board CT (conductivity-temperature) and pressure sensors, along with a computer running autonomy software that can process the data and adaptively redirect the vehicle without an operator in the loop. The choice to use AUVs rather than satellite data, moorings, or CTD casts from a ship for this ex- 
periment gave us the flexibility to capture the exact data set we needed using the AUVs' abilities to conduct autonomous and adaptive environmental sampling in real time, such as thermocline tracking (the thermocline and pycnocline depths are coincident in our AUVs' shallow-water operations region). Autonomous coordination is also possible between multiple AUVs, allowing (in this case) one AUV to travel at the pycnocline depth to collect a data set that is likely to contain internal waves while the other AUV travels well below the pycnocline along the same horizontal track as the first AUV to collect a 'ground truth' data set where internal waves are unlikely to occur.

Using AUVs for internal wave detection is a relatively novel approach. However, the approach presented here is not the first to employ AUVs for this task. Work was done by Zhang et al. in 2001 [76] on spectral classification of internal waves based on vertical flow velocity data from an AUV-mounted ADV (acoustic Doppler velocimeter) during the 1998 Labrador Sea Convection Experiment. In that experiment, the AUV was driven in a predetermined horizontal square pattern at two depths in the upper mixed layer to collect data, which was processed and compared with spectra from an ocean model of the Labrador Sea region containing internal waves. Although no internal waves were found in the vertical velocity spectra, results suggest that significant convection was present in the experimental region. Work has also been done by Cazenave in his 2008 Master's thesis [77] on internal wave detection using the CTD sensor on an AUV, similarly examining the temperature spectra. Cazenave's experiment took place throughout 2007 in Monterey Bay, CA, through which energetic internal waves are known to pass daily (they have been imaged by satellite). He uses a single AUV that follows a predefined track line between two waypoints in horizontal space while yo-yoing in depth around a set temperature range that is expected to traverse the thermocline in depth. Perturbations in the isotherms and spectral analysis were then used to quantify the internal waves.

The approach in this work is similar to Cazenave's (and different from that of Zhang et al.) in that it uses CT and pressure data, since CTD (or CT and pressure) sensors are standard on most AUVs. The primary difference from the experiments of Cazenave and Zhang et al. lies in the adaptive and autonomous approach to sampling the environment, and by using multiple AUVs in collaboration to capture synoptic data sets. Also, instead of looking for characteristic vertical velocity modes of internal waves predicted by ocean models (as this will vary from one body of water to the next and requires learning and running an ocean model specific to each location) as done in [76], a direct signal processing approach is taken which is similar to that in [77] to detect the primary frequencies and wavelengths of any potential internal waves propagating along the thermocline interface. In the experiment described below, what Cazenave's thesis work identified as future work to make internal wave sampling with AUVs more autonomous, collaborative, and 
environmentally adaptive was essentially implemented.

On August 13, 2010, we conducted the Internal Wave Detection Experiment (a single-day experiment in the larger GLINT '10 experiment) in the northern coastal basin of the Tyrrhenian Sea bordered by the Tuscan Archipelago and the western coast of Italy (see Fig. 3-12). Based on historical satellite data and basic bathymetric data from this region in the summer, we expected to see a water depth of less than 200 $\mathrm{m}$ in the operation region shown in Fig. 3-12 (it was actually about $110 \mathrm{~m}$ deep at that location) and sea surface temperatures of about $24^{\circ} \mathrm{C}$ with temperatures around $20^{\circ} \mathrm{C}$ at $20 \mathrm{~m}$ depth and around $14^{\circ} \mathrm{C}$ near the sea floor, suggesting summertime stratification that had the potential to sustain internal waves [78-80]. According to Turner [68], internal waves propagating along the oceanic thermocline typically have periods of a few minutes, whereas deep ocean internal waves may have periods of up to many hours. Thus, it is expected to see internal waves with periods of a few minutes along the thermocline in the Tyrrhenian Sea. This location was chosen due to the availability of ship and AUV resources already deployed for the longer GLINT '10 AUV autonomy experiments. In addition, when researching the possibility of internal waves in the Tuscan Archipelago basin, all but one scientist interviewed at the NATO Undersea Research Centre (NURC) in La Spezia, Italy, claimed that observations of any internal waves in the basin were unlikely, but none could provide any evidence for this. Also, no published literature was found on the subject of the presence of internal waves in the Tuscan Archipelago basin, so it was decided to conduct the Internal Wave Detection Experiment there.

\subsection{A Novel Approach to Implementing Internal Wave Detection}

During the Internal Wave Detection Experiment, the use of multiple AUVs communicating (fully through acoustic communication while submerged) and interacting with each other and the environment autonomously in real time to collect a synoptic environmental data set was demonstrated. The resulting environmental data set would be otherwise incomplete using only one AUV. The two AUVs that were used each belonged to a different research group and were of different manufacture. Thus, we were also able to demonstrate that not only could multiple AUVs of different types work together using a common on-board autonomy structure, but that both research groups (the Massachusetts Institute of Technology's Laboratory for Autonomous Marine Sensing Systems from Cambridge, MA, USA, and the researchers and AUV team from NURC) could collaborate their efforts to advance the quality and quantity of data collected.

Acoustic communication is used nearly exclusively during our AUV operations for AUV-to-AUV and AUV-to-ship/lab (via gateway buoy or Towfish modem) scientific and navigational data exchange in virtu- 
ally real time (delays on the order of seconds to minutes). The software behind this is the Goby Underwater Autonomy Project through the pAcommsHandler interface for the Mission Oriented Operating Suite (MOOS) $[6,7]$ autonomy system. A common suite of autonomy software is used on board each AUV and the topside (operator) computers to tie together all of the pieces to allow the AUVs to collaborate autonomously with each other and adapt to the environment. Both the MIT and NURC groups use the MOOS and the IvP Helm, which work in conjunction to make the AUVs carry out a variety of autonomy behaviors. These behaviors autonomously and adaptively reason over AUV heading, speed, and depth, depending on the behaviors that the operators set as active on each AUV $[1,2]$.

\subsubsection{Hardware Platforms}

To deploy the AUV missions (detailed below) for the Internal Wave Detection Experiment, two actively propelled AUVs and an acoustic communications 'gateway' buoy were required. In addition, 10 thermistors were attached to the wet line on the buoy to create a thermistor chain. The AUV command and control center, or 'topside,' was located in the lab on the NRV Alliance, positioned within a $5 \mathrm{~km}$ range from the deployed AUVs and buoy for the experiment's duration.

The Bluefin 21" AUV named Unicorn is operated by our Laboratory for Autonomous Marine Sensing Systems at the Massachusetts Institute of Technology (MIT). It has a 21" hull diameter and was $~ 3 \mathrm{~m}$ in length in the GLINT '10 experiment configuration shown in Fig. 4-1. Unicorn's speed range for best motion control is $1.0-1.8 \mathrm{~m} / \mathrm{s}$, though it is often commanded to travel at $1.5 \mathrm{~m} / \mathrm{s}$ (although this varies if Unicorn is running according to autonomous adaptation behaviors) and has poor vertical stability below $1.3 \mathrm{~m} / \mathrm{s}$. Navigation instrumentation for Unicorn consists of a Leica DMC-SX Magnetic Compass and a Crossbow AHRS (attitude heading reference sensor) resulting in a navigational error of about $1 \%-5 \%$ of the distance traveled between acquiring GPS position fixes. This navigational error assumes Unicorn has constant DVL (Doppler velocity log) bottom-lock, has completed a compass hard iron/soft iron calibration, has completed a compass star maneuver (for compass calibration in the water), and the Bluefin software on board has done some calibrations and mathematics to improve the navigational accuracy to this point. As such, Unicorn must surface for a GPS position fix every 30 minutes, during which time it accumulates about 50-100 $\mathrm{m}$ of navigational error. Other instrumentation on Unicorn during GLINT ' 10 consisted of a CT sensor, a pressure sensor, and an acoustic modem with transducer.

The Ocean Explorer (OEX) AUV named Harpo is operated by a group at the NATO Undersea Research Centre (NURC) based in La Spezia, Italy. It has a 21 " hull diameter and was $4.3 \mathrm{~m}$ in length in the GLINT 


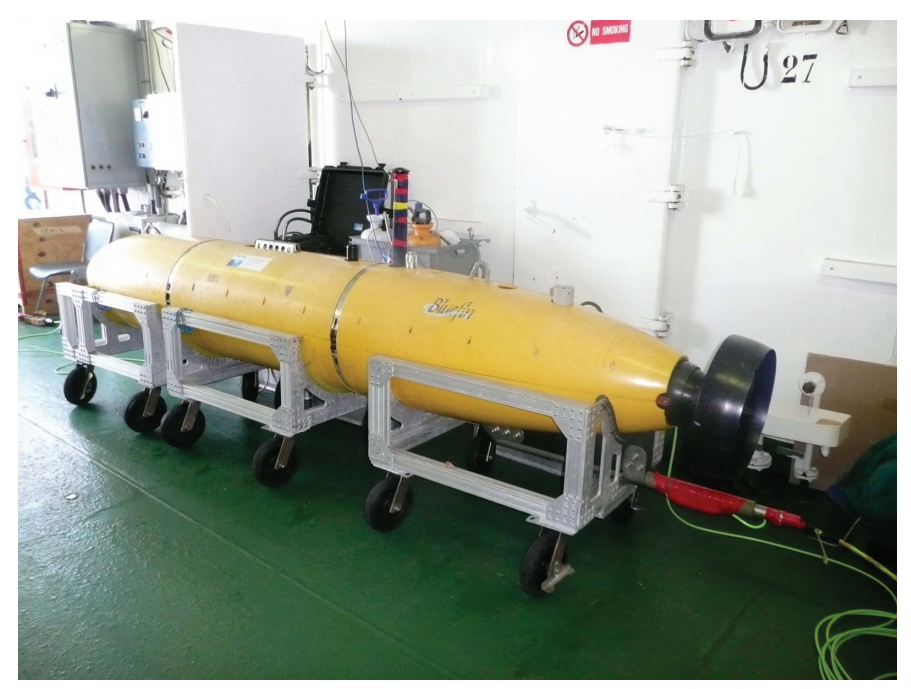

Figure 4-1: The Bluefin 21" Unicorn AUV operated by the MIT Laboratory for Autonomous Marine Sensing Systems.

'10 experiment configuration shown in Fig. 4-2. Harpo's maximum speed is quoted at about $1.2 \mathrm{~m} / \mathrm{s}$, though it is often run slower to conserve battery power. For navigation, Harpo runs an IMU (inertial measurement unit) in conjunction with an acoustic DVL with bottom-lock that has little position drift (under $100 \mathrm{~m}$ ) over the course of the day (often about 7 hours of runtime) after completing an in-water navigation alignment each morning [81]. This means that Harpo does not need to surface for GPS position fixes during experiments. Other instrumentation on Harpo during GLINT '10 consisted of a CTD sensor, and two acoustic modems with transducers.

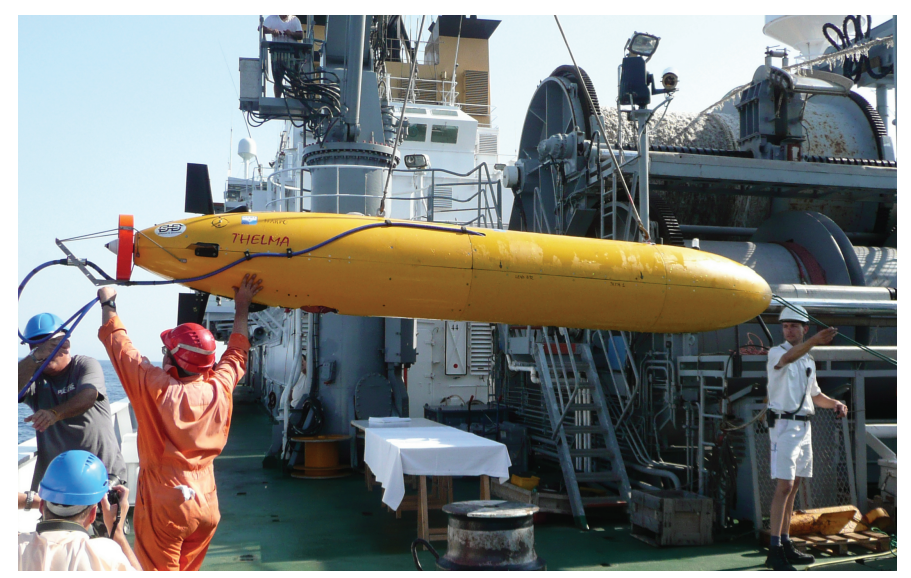

Figure 4-2: The NURC OEX-Harpo AUV used during GLINT '10. This AUV communicates with the ship and the MIT Unicorn AUV via acoustic communication (underwater). It also carries a GPS for positioning.

Both AUVs were equipped with a WHOI WH-BT-2 $28 \mathrm{kHz}$ acoustic transducer [8] and on board pay- 
load computers running Linux operating systems with MOOS and IvP Helm autonomy software and the pAcommsHandler acoustic communication polling handler, similar to that used on the topside computers.

The MIT topside maintained radio frequency (RF) communication with the gateway buoy through a Freewave antenna mounted outside the upper deck of the NRV Alliance and acoustic communication with the AUVs via the acoustic modem transducer and hydrophone array hanging from the gateway buoy. The NURC topside maintained acoustic communication with Harpo via a Towfish acoustic modem transducer hanging in the water over the side of the ship. Both groups' topside computers included a Google Earth Ocean Viewer (GEOV) situational display of all AUVs, buoys, ships, and instruments in the water as in Fig. 3-13 [63], as well as the AUV command and control software (MOOS and IvP Helm) and pAcommsHandler acoustic message encoding/decoding and queuing/sending code.

The gateway buoy was a Micro-modem VSW Modem Buoy built by the Woods Hole Oceanographic Institution (WHOI) Acoustic Communications Group [8]. It was equipped with a GPS unit and Freewave RF antenna on the surface expression and a hanging wet cable of approximately $30 \mathrm{~m}$ length equipped with a 4-hydrophone array (for high-rate communication) and an acoustic modem transducer at the bottom. The buoy itself was stationed at the center of the AUV loiter patterns during each mission.

The 10 thermistors were placed along the buoy's wet cable at approximately $3 \mathrm{~m}$ spacing and sampled the temperature every 30 seconds as a ground-truth for the presence of internal waves in the region.

\subsubsection{AUV Missions}

This experiment initially consisted of three AUV missions, however only the first two were completed due to time constraints and operational difficulties. From an early morning ship CTD cast and some pre- and midexperiment yo-yos through the water column using Unicorn, the peak temperature change of the thermocline was noted at $10 \pm 1 \mathrm{~m}$ depth throughout most of the day. It should also be noted that performing horizontal loiter patterns on a radius of $\mathrm{O}(500 \mathrm{~m})$ may be considered a point measurement relative to the scale of the large basin bounded by the Tuscan Archipelago, though on a local scale the pentagonal shape of the loiters (each of the 5 legs providing wave information from a different direction) has potential to enable us to determine the direction of travel of internal waves. A screen shot of the situational display from Mission 2 is shown in Fig. 3-13 to help visualize the mission layouts, and details of each mission are presented in Tables 4.1, 4.2, and 4.3. Descriptions of the adaptive autonomy behaviors used follow in Section 4.5.3. 
Table 4.1: Mission 1

\begin{tabular}{|l|l|}
\hline Description & $60 \mathrm{~m}$ depth loiter $\& 10 \mathrm{~m}$ depth trail loiter \\
\hline \hline OEX-Harpo & \\
\hline Horizontal pattern & pentagonal loiter, $550 \mathrm{~m}$ radius, clockwise travel \\
\hline Depth & $60 \mathrm{~m}$, constant depth behavior \\
\hline Speed & $1.3 \mathrm{~m} / \mathrm{s}$ \\
\hline \hline BF21-Unicorn & $\begin{array}{l}\text { trail Harpo at } 150 \mathrm{~m} \text { range, } 180^{\circ} \text { relative trail angle (directly be- } \\
\text { hind Harpo })\end{array}$ \\
\hline Horizontal pattern & $\begin{array}{l}\text { at thermocline } \sim 10 \mathrm{~m}, \text { adaptive constant depth behavior } \\
\text { (changed to } 12 \mathrm{~m}, \text { constant depth behavior during experiment) }\end{array}$ \\
\hline Depth & $1.3 \mathrm{~m} / \mathrm{s}$ (adaptive to trail Harpo) \\
\hline Speed & \\
\hline
\end{tabular}

Table 4.2: Mission 2

\begin{tabular}{|l|l|}
\hline Description & $\begin{array}{l}10 \mathrm{~m} \text { depth loiter \& adaptive yo-yo trail loiter (depth-adaptive to } \\
\text { thermocline) }\end{array}$ \\
\hline \hline OEX-Harpo & pentagonal loiter, $550 \mathrm{~m}$ radius, clockwise travel \\
\hline Horizontal pattern & $\begin{array}{l}\text { at thermocline } \sim 10 \mathrm{~m}, \text { adaptive constant depth behavior } \\
\text { (changed to } 12 \mathrm{~m}, \text { constant depth behavior during experiment) }\end{array}$ \\
\hline Depth & $1.3 \mathrm{~m} / \mathrm{s}$ \\
\hline Speed & $\begin{array}{l}\text { trail Harpo at } 150 \mathrm{~m} \text { range, } 180^{\circ} \text { relative trail angle (directly be- } \\
\text { hind Harpo) }\end{array}$ \\
\hline \hline BF21-Unicorn & $\begin{array}{l}\text { adaptive yo-yo (toggle depth) behavior within thermocline depth } \\
\text { range (calculated by pEnvtGrad [3]), beginning with 7-70 m dive } \\
\text { range }\end{array}$ \\
\hline Horizontal pattern & $1.3 \mathrm{~m} / \mathrm{s}$ (adaptive to trail Harpo) \\
\hline Depth & \multicolumn{2}{|l}{} \\
\hline Speed &
\end{tabular}

\subsubsection{MOOS Processes and IvP Helm Autonomy Behaviors}

As previously mentioned, MOOS is the underlying autonomy software on board the AUVs and on the topside operators' computers. MOOS is essentially a publish-subscribe architecture that passes messages between autonomy processes and behaviors on board each AUV, as well as through the water between the AUVs and the topside computer [1]. The brains behind the autonomy lie in the IvP Helm code that is integrated into MOOS to implement the use of autonomy behaviors (e.g., vertical yo-yos, trail-an-AUV, horizontal racetracks, safety behaviors) on the AUVs. These behaviors optimize over an AUV's heading, speed, and depth 
Table 4.3: Mission 3

\begin{tabular}{|l|l|}
\hline Description & $\begin{array}{l}\text { concentric loiters at } 10 \mathrm{~m} \text { depth \& depth-adaptive to thermocline } \\
\text { (adaptive yo-yo), outer AUV trails (not completed) }\end{array}$ \\
\hline \hline OEX-Harpo & pentagonal loiter, $450 \mathrm{~m}$ radius, clockwise travel \\
\hline Horizontal pattern & at thermocline $\sim 10 \mathrm{~m}$, adaptive constant depth behavior \\
\hline Depth & $1.0 \mathrm{~m} / \mathrm{s}$ \\
\hline Speed & $\begin{array}{l}\text { trail Harpo at } 150 \mathrm{~m} \text { range, } 315^{\circ} \text { relative trail angle } \text { (off Harpo's } \\
\text { stern and to port, resulting in } 550 \mathrm{~m} \text { radius outer loiter) }\end{array}$ \\
\hline \hline BF21-Unicorn & $\begin{array}{l}\text { adaptive yo-yo (toggle depth) behavior within thermocline depth } \\
\text { range (calculated by pEnvtGrad), beginning with } 7-70 \text { m dive } \\
\text { range }\end{array}$ \\
\hline Horizontal pattern & $1.5 \mathrm{~m} / \mathrm{s}$ (adaptive to trail Harpo) \\
\hline Depth &
\end{tabular}

to control its motion through the water, depending on what behavior is being followed $[1,2]$. The MOOS processes and behaviors most relevant to the Internal Wave Detection Experiment are described below.

\section{Environmental Gradient Determination Process: pEnvtGrad}

One process that is run using MOOS is the environmental gradient determination process, pEnvtGrad, used to perform thermocline tracking and similar environmentally adaptive behaviors. This process—-described in detail in Chapter 3 and [3] — monitors and sorts an AUV's CTD data, using the data to calculate vertical gradients of temperature $(|\partial T / \partial z|)$ through the water column, the depth range (upper and lower bounds) covered by the thermocline, and the depth at which the thermocline gradient is strongest (maximum $|\partial T / \partial z|$ ). These calculated values are then published to the MOOS database on the AUV to be used to guide environmentallyfocused adaptive behaviors, such as the adaptive yo-yo (toggle depth) behavior and the adaptive constant depth behavior described below. pEnvtGrad is run concurrently with either of these depth-adaptive behaviors. The calculated values are also used by other MOOS processes and behaviors that need to know environmental information, and the thermocline boundary and peak gradient values are sent acoustically to other AUVs as informational data and to the topside for monitoring by the AUV operators. pEnvtGrad also calculates analogous values for profiles of sound speed and density, which are derived from temperature, salinity, and pressure measurements. A conceptual sketch of the adaptive thermocline tracking process using pEnvtGrad is shown in Fig. 3-1. The AUV performs an initial yo-yo dive from the surface to as deep as is allowable while collecting temperature (and/or salinity and pressure) data. The water column is divided into many depth bins, 
over which temperature measurements are averaged, then the vertical gradients of temperature $(\partial T / \partial z)$ are calculated between depth bins. The magnitude of the average of the vertical temperature gradients is set as the threshold value, and any depth bin in which $|\partial T / \partial z|$ exceeds the threshold value is flagged as being within the thermocline. Thus, an upper and lower depth bound for the thermocline region can be defined, as well as the peak thermocline depth as the depth bin with the maximum $|\partial T / \partial z|$. More detail on the algorithms used by pEnvtGrad and related field trials can be found in Chapter 3 and [3].

In the GLINT '10 Internal Wave Detection Experiment, pEnvtGrad was employed by Unicorn in Mission 2 to obtain a three-dimensional data set of the temperature variations in the operational region, which will ultimately be used to analyze internal wave amplitudes.

\section{Adaptive Yo-Yo (Toggle Depth) Behavior}

The adaptive yo-yo (toggle depth) IvP Helm behavior, BHV_ToggleDepth, controls the desired depth of an AUV. It sets the desired upper and lower depth boundaries of a vertical yo-yo (or sawtooth) pattern for the AUV based on the upper and lower depth boundaries of the thermocline, as determined by pEnvtGrad (during the Internal Wave Detection Experiment). That is, as the thermocline boundary depths change over the course of a thermocline tracking mission (as in Mission 2), BHV_ToggleDepth adapts the boundaries of the AUV's yo-yo to match those of the thermocline in real time by toggling the commanded depth between these two bounds to ensure that the desired depths are achieved. BHV_ToggleDepth can be active while performing any horizontal deployment pattern (e.g., racetrack, loiter, zigzag, track-and-trail).

\section{Adaptive Constant Depth Behavior}

The adaptive constant depth IvP Helm behavior uses BHV_ConstantDepth to set a single desired depth for an AUV to swim at based on the peak thermocline depth (the depth of maximum temperature change per unit depth) calculated by pEnvtGrad. As the peak thermocline depth shifts up or down in the water column, the desired AUV depth commanded by BHV_ConstantDepth is automatically updated to match it, autonomously adapting to the changes in the environment in real time. Unfortunately, swimming an AUV at the peak thermocline depth results in very poor acoustic communications to and from that AUV, so we opted to command the AUVs to a constant depth a couple of meters below the peak of the thermocline with the non-adaptive mode of BHV_ConstantDepth such that we could continue to monitor the AUVs regularly throughout the missions and so that the AUVs could communicate with each other to perform the track-and-trail behavior. 


\section{Track-and-Trail Mode}

The track-and-trail mode puts the trailing AUV into 'TRAIL' mode, shadowing a leading AUV (or any leading platform for which the trailing AUV receives position updates via acoustic messages) in the horizontal plane. The relative bearing and trailing distance from the trailing AUV to the leading AUV must be set by the operator, and the depth modes (e.g., constant depth, adaptive constant depth, adaptive yo-yo toggle depth) of the two AUVs are set independently of each other and independently of being in TRAIL mode. The leading AUV is not in TRAIL mode (unless it is trailing yet another platform) and leads the mission in the horizontal plane.

\section{pAcommsHandler}

The Goby Underwater Autonomy Project's MOOS interface, pAcommsHandler, controls the queuing and sending of data through the underwater acoustic channel on all acoustically-communicating platforms in this work and is crucial to all of the AUV missions. It encodes the data (science data, navigation data, status data, etc.) on one node (AUV, topside, or gateway buoy), slots the encoded message into the polling queue, initializes the acoustic transmission, and decodes the data as it is received on another node running pAcommsHandler [6,7]. This all occurs while missions are underway on the AUVs, resulting in virtually real-time data transmission. This real-time communication is necessary when there are multiple AUVs in the water that need to know information about one another to collaborate their motions and avoid collisions. Finally, it is also important to the topside operators, who want real-time data updates to monitor the progress and autonomy behaviors of the AUVs and to monitor the changes in their environment and scientific data over the course of an AUV mission.

\subsection{Data \& Results}

This section compiles not only results of the data processing to determine the internal wave frequencies and whence they originated, but also some of the unexpected effects that the field deployment had on the planned missions and resulting data. These effects are largely due to physical constraints of the AUVs and instruments and imposed effects of a dynamic ocean environment on conducting AUV missions. A brief description of the oceanographic conditions on the day of the experiment is presented first. 


\subsubsection{Oceanographic Conditions}

Fig. 4-3 shows the morning and afternoon sound speed, temperature, salinity, and density profiles from a CTD cast from the NRV Alliance on August 13, 2010, in the GLINT '10 operation area. The water depth at the CTD sample locations (and much of the operation area) was just over $110 \mathrm{~m}$. Here a warm isothermal mixed layer can be seen near the surface of approximately $10 \mathrm{~m}$ depth and $24^{\circ} \mathrm{C}$ resulting in a strong thermocline at about $10 \mathrm{~m}$ depth. The temperature then drops suddenly with depth to about $19^{\circ} \mathrm{C}$, then tapers off to about $14^{\circ} \mathrm{C}$ by $60 \mathrm{~m}$ depth, below which the water remains isothermal. The steep thermocline near $10 \mathrm{~m}$ depth suggests that internal waves would be most prominently observable at that depth, if they existed. It should be noted that the high frequency variations in salinity over depth are likely due to the sensitivity of the conductivity sensor on the CTD to the rapid changes in temperature between 9 and $60 \mathrm{~m}$. Sound speed was calculated using the Mackenzie sound speed equation [65]. Density was calculated from the Unesco 1983 equation of state for sea water [67].

\subsubsection{Mission Execution}

At the beginning of the Internal Wave Detection missions, the shallow-depth AUV was commanded to swim at the depth of the maximum gradient of the thermocline ( $\sim 10 \mathrm{~m}$ depth). This resulted in extremely poor acoustic communication observed between the shallow AUV (Unicorn, for the first mission) and the topside (via the gateway buoy) due to the fluctuating refraction direction of propagating sound waves in the steepest region of the thermocline (depth of maximum $|\partial T / \partial z|)$. With Unicorn traveling at $10 \mathrm{~m}$ depth, 3/14 (21\%) of the acoustic messages sent by Unicorn to the topside were received on the topside, while 19/38 (50\%) of them were received on the topside with Unicorn traveling at $12 \mathrm{~m}$ depth (acoustic communication performance values based on rate 0 FH-FSK (frequency-hopped frequency shift keying) messages sent from Unicorn to the gateway buoy, data courtesy of Toby Schneider, MIT). Subsequent missions had the depth of the shallow (constant depth) AUV changed to swim at $12 \mathrm{~m}$ — just below the peak gradient of the thermocline-from the start of the mission to avoid losing contact with that AUV.

The next challenge faced during deployment was a difference in speed ranges achievable by Unicorn and Harpo. This was significant because, in order for Unicorn to trail behind Harpo without overtaking Harpo, Unicorn had to slow to its minimum speed of $1.3 \mathrm{~m} / \mathrm{s}$ while Harpo had to travel at $1.3 \mathrm{~m} / \mathrm{s}$, just above Harpo's maximum quoted speed. When Unicorn slowed below $1.3 \mathrm{~m} / \mathrm{s}$ to remain at a safe distance behind Harpo, its depth control degraded and it was observed to fluctuate involuntarily, or 'porpoise,' in depth by up to \pm 0.8 $\mathrm{m}$ in a periodic manner, adding a detectable temperature fluctuation to its data set. Upon processing, the 


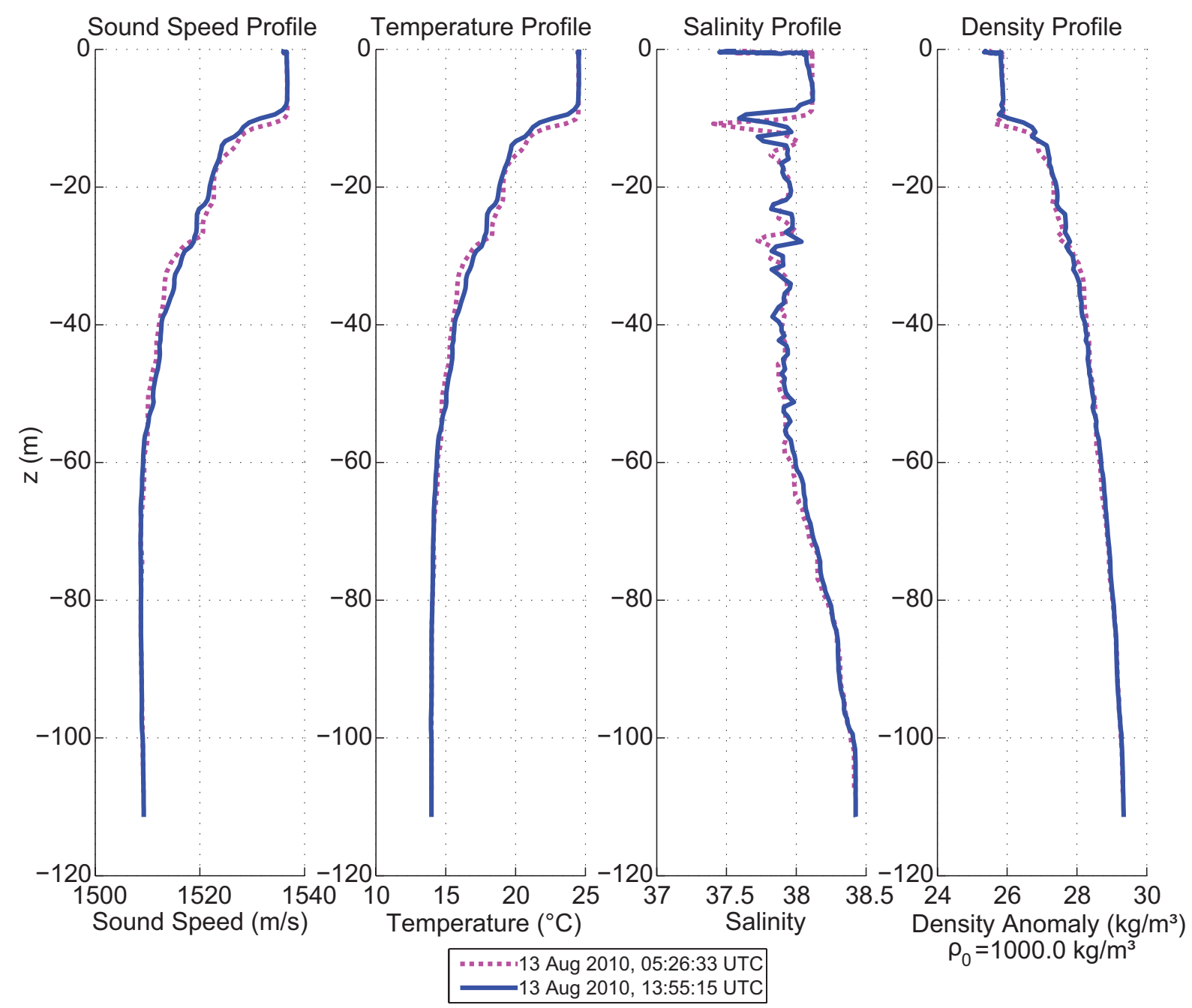

Figure 4-3: Morning and afternoon sound speed, temperature, salinity, and density profiles from a CTD cast from the NRV Alliance on August 13, 2010.

power spectral density peaks at the dominant frequencies of Unicorn's porpoising $\left(P S D_{\text {Depth_Unicorn }}\right)$ were subtracted from the temperature spectrum $\left(P S D_{\text {Temp_Unicorn }}\right)$ to minimize their influence on the results. The resulting 'pure' temperature spectrum $\left(P S D_{T e m p \_p u r e}\right)$ is calculated as follows:

$$
P S D_{\text {Temp_pure }}=P S D_{\text {Temp_Unicorn }}-P S D_{\text {Depth_Unicorn }} .
$$

In the future, the porpoising could be avoided by adjusting the controller gains on Unicorn for smoother operation at slower speeds (there was no access to this option or time to implement and test it for these missions). Alternatively, a new loiter behavior could be written to incorporate a horizontal zigzag pattern on each loiter leg to slow down Unicorn's forward progress, but this option was not available at the time and 
the idea was to collect data along the 5 fixed headings of the pentagonal loiter to eventually back out the direction of travel of any internal waves (beyond the scope of this thesis work). During Mission 2, Unicorn's minimum speed was not a problem because it was slowed in horizontal speed by the yo-yo depth excursions it was performing.

During the second mission in which Unicorn was adapting its yo-yo depth range to focus around the thermocline, hysteresis was observed in the temperature data (see Fig. 4-4). As Unicorn ascended through the 12-meter depth mark, the temperature was consistently observed to be lower than the AUV's subsequent descent through the 12-meter depth mark. In Unicorn, the CT sensor is mounted on top, mid-way between the nose and tail of the AUV, and the pressure sensor (giving depth readings) is mounted in the bottom of the aft section of the AUV. Thus, if there were any appreciable lag between sensor readings of temperature and pressure at $12 \mathrm{~m}$, the temperature reading at $12 \mathrm{~m}$ would be expected to be higher on the ascent (CT sensor at the mid-section is higher in the water column than the aft pressure sensor) and lower on the descent, which is the opposite of what had been observed. The Sea-Bird Electronics, Inc., model SBE 37-SI CT sensor on Unicorn has an acquisition time of 1.0-2.6 seconds/sample [82], which is comparable to the $\sim 1.5 \mathrm{~s}$ it takes the pressure sensor to catch up in depth to where the previous temperature measurement was taken, which may account for some of the discrepancy, and thus, the hysteresis. The resolution of the temperature sensor on Unicorn is specified as $0.0001{ }^{\circ} \mathrm{C}$ [82], while the Paroscientific, Inc., Digiquartz depth sensor resolution is at $0.1 \mathrm{~mm}$ or better with and accuracy of $0.02 \%$ or less and hysteresis $\leq \pm 10 \mathrm{~cm}$ [83]. Thus, this temperature fluctuation is not due to the resolution of the temperature or depth sensor. This leaves the only probable explanation of the temperature fluctuation as hysteresis between the CT and pressure sensors due to the slow acquisition time of the temperature sensor and the hysteresis in the pressure sensor. One way to adjust for this in post-processing is to find the average temperature difference between each instance of shoaling and diving through the 12-meter depth mark, and add (subtract) half the difference to (from) the temperature measurement on the ascent (descent). The best way to prevent the majority of this hysteresis is to use a pumped CTD (or CT plus depth) sensor instead of a flow-through CT sensor plus depth sensor. In this case, the CT and depth sensors were the instruments available on the AUVs, and the mounting locations are fixed.

The thermistor chain was deployed throughout both successful AUV missions, however it was only sampling at a 30-second interval compared to the approximately $10 \mathrm{~Hz}$ and $4 \mathrm{~Hz}$ sampling frequencies of Unicorn and Harpo, respectively. This means that the thermistor data spectra are resolved for a much lower frequency range than the spectra from the AUVs' data (see Figs. 4-7, 4-9, and 4-11), allowing us to detect any possible lower-frequency internal waves. 

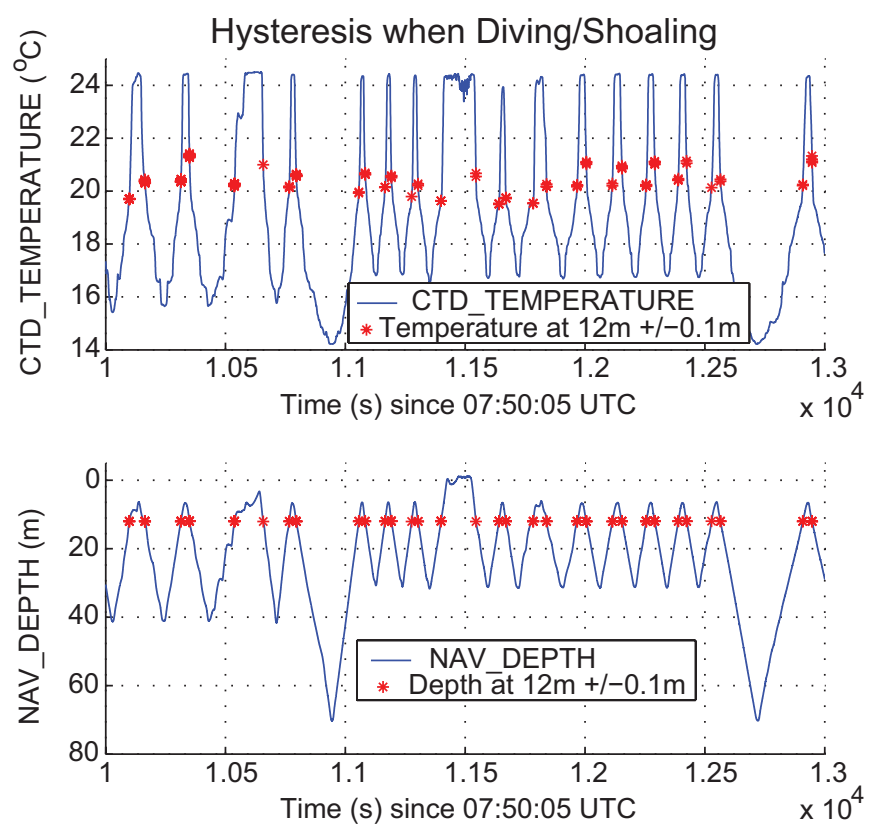

Figure 4-4: Hysteresis is seen in Unicorn's temperature data (CTD_TEMPERATURE) while preforming yoyos through the water column. NAV_Z values are the negative of Unicorns measured depth values. The stars signify temperature and depth measurements taken when Unicorn is at $12 \pm 0.1 \mathrm{~m}$ depth. It has been verified that the $\pm 0.1 \mathrm{~m}$ depth range allowed is not the cause of the hysteresis.

Finally, atmospheric weather conditions can also affect underwater measurements through surface interactions of wind and waves. From approximately 0900-0930 UTC, or 1100-1130 local time ( 30-60 min into Mission 1), a storm system passed over the ship and AUV operation area. Storms frequently sustain higher winds than clear-weather conditions, and introduce an influx of fresh water to the otherwise salty sea surface. Depending on the severity of the storm, its effects on the underwater environment may lag the storm and persist from hours to weeks after the storm has passed. In this case, the storm only covered a local area of about $200 \mathrm{~km}^{2}$ with squalls of very heavy rain, and it did not appear to cause an appreciable change in the temperature at the thermocline immediately following the storm's passing. Over the course of the the entire day (end of Mission 1 and through Mission 2, about 4.5 hours), however, there was an overall decrease in temperature of $\sim 0.5^{\circ} \mathrm{C}$ by the end of Mission 2 . It is unlikely that this temperature decrease is due to the storm, since a deluge of $10 \mathrm{~cm}$ of water at $14{ }^{\circ} \mathrm{C}$ advected into the surface mixed layer $\left(10 \mathrm{~m}\right.$ deep, $\left.24^{\circ} \mathrm{C}\right)$ over the storm's area would only decrease the mixed-layer temperature by about $0.1{ }^{\circ} \mathrm{C}$ or less. Thus, it is more likely that this drop in mixed-layer temperature is due to surface cooling as the post-storm sunshine waned going into the mid-afternoon (local time). 


\subsection{Analysis}

Since the goal of the Internal Wave Detection Experiment was to detect the presence of internal waves in the basin of the Tyrrhenian Sea bounded by the Tuscan Archipelago (or more specifically bounded by the small GLINT '10 operational area), the data analysis was approached from a signal processing standpoint once a baseline for temperature fluctuations was established. In order to preserve any transient frequency peaks in the temperature spectra that may be representative of soliton internal waves, no data windowing was done to generate the Power Spectral Density (PSD) plots in this section.

\section{Mission 1}

Mission 1 lasted from 0833-0853 UTC with Unicorn at $10 \mathrm{~m}$, and from 0913-1007 UTC with Unicorn at $12 \mathrm{~m}$. Between these times, Unicorn was at $13 \mathrm{~m}$ for $20 \mathrm{~min}$. However, at this time the $20 \mathrm{~min}$ data set is set aside in favor of focusing on the time spans in which Unicorn was closest to the thermocline depth. Harpo was at $60 \mathrm{~m}$ from $0818-1120$ UTC.

From Mission 1, Harpo's temperature data at $60 \mathrm{~m}$ depth (significantly below the thermocline region) exhibited a baseline of small fluctuations in temperature $\left( \pm 0.3^{\circ} \mathrm{C}\right)$ as seen in Fig. 4-5. There is insufficient data, given the temporal sparsity of temperature profiles passing through $60 \mathrm{~m}$ depth and the small temperature change per unit depth at $60 \mathrm{~m}$, to determine whether variations in these temperature data are due to internal waves or not.

In contrast, Unicorn's temperature data at $10 \mathrm{~m}$ and $12 \mathrm{~m}$ during Mission 1 revealed a number of peakenergy frequencies above the noise floor in its PSD plots (Fig. 4-6). Due to the porpoising motion of Unicorn during Mission 1, the PSD of Unicorn's depth was subtracted from the PSDs of temperature to get the 'pure' temperature spectra at 10 and $12 \mathrm{~m}$ using Equation 4.1. The frequencies and PSDs of the pure temperature spectrum's local maxima at $10 \mathrm{~m}$ and $12 \mathrm{~m}$ depth are plotted as stars in Fig. 4-6. This is a satisfactory approach, since the lack of windowing captures frequencies of internal wave packets or solitons that traverse the operational region on a time scale significantly shorter than our overall mission length. To show the time variation of the spectra, we have also analyzed the temperature measurements at $10 \mathrm{~m}$ and $12 \mathrm{~m}$ using the spectrogram shown in Fig. 4-7. The broadband blips in energy at 30-minute intervals are a result of Unicorn surfacing at those times to acquire a GPS position fix. There appears to be a very weak but persistent narrow-band peak around $4.0 \mathrm{~Hz}$ in the $12 \mathrm{~m}$ spectrogram, which is well above the possible internal wave frequencies and probably due to sensor noise. Other potentially interesting peaks appear below $0.3 \mathrm{~Hz}$ at about $650-1150 \mathrm{~s}$ in the $10 \mathrm{~m}$ spectrogram and at about 700-800, 1250, 1700-1800, 2000-2500, 2550- 

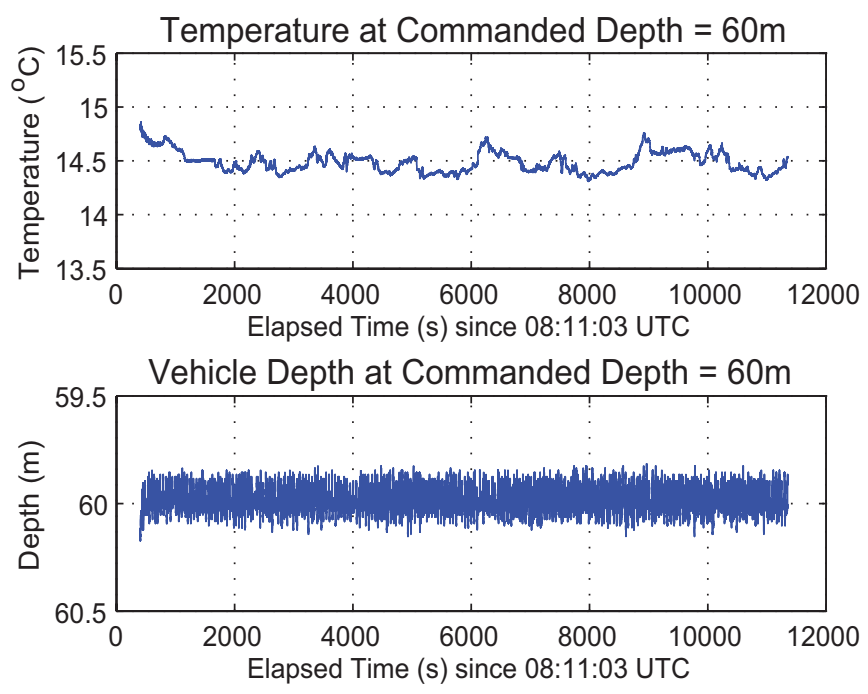

Figure 4-5: Temperature and depth times series of data from Harpo at $60 \mathrm{~m}$ depth during Mission 1. This is used as a baseline measurement of the temperature fluctuations in the relatively density-homogeneous layer well below the thermocline.

2650 , and $2950-3000 \mathrm{~s}$ in the $12 \mathrm{~m}$ spectrogram, some of which may belong to internal soliton waves. None of Unicorn's low-frequency $(<0.05 \mathrm{~Hz})$ energy peaks in the spectrogram are well distinguished from one time point to the next, thus it has been chosen to leave out a low-frequency zoomed-in version of this plot.

\section{Mission 2}

Mission 2 lasted from 1139-1250 UTC with Harpo at 12 m, though Unicorn tracked the thermocline adaptively from 1009-1327 UTC.

With a below-thermocline baseline data set established at $60 \mathrm{~m}$ during Mission 1, Harpo was re-tasked to swim at $12 \mathrm{~m}$ depth for Mission 2 to track just below the peak thermocline gradient as Unicorn did in Mission 1. Unicorn was re-tasked to perform adaptive thermocline tracking while autonomously trailing Harpo. Due to temporal separation of Missions 1 and 2, Harpo captured the passing of internal waves in its temperature data at $12 \mathrm{~m}$ which exhibited somewhat different peak frequencies than captured by Unicorn in Mission 1. A plot of Harpo's pure temperature spectrum at $12 \mathrm{~m}$ is shown in Fig. 4-8 with the peak PSD frequencies plotted as red stars. To show the time variation of the spectra, we analyzed the temperature measurements at $12 \mathrm{~m}$ in the form of the spectrogram shown in Fig. 4-9. Harpo did not need to surface for GPS position fixes, so there are no broadband peaks at 30-minute intervals like the ones seen for Unicorn in Fig. 4-7. There again appears to be a very weak but persistent narrow-band peak, only this time it is around $1.7 \mathrm{~Hz}$ (beyond the axes 


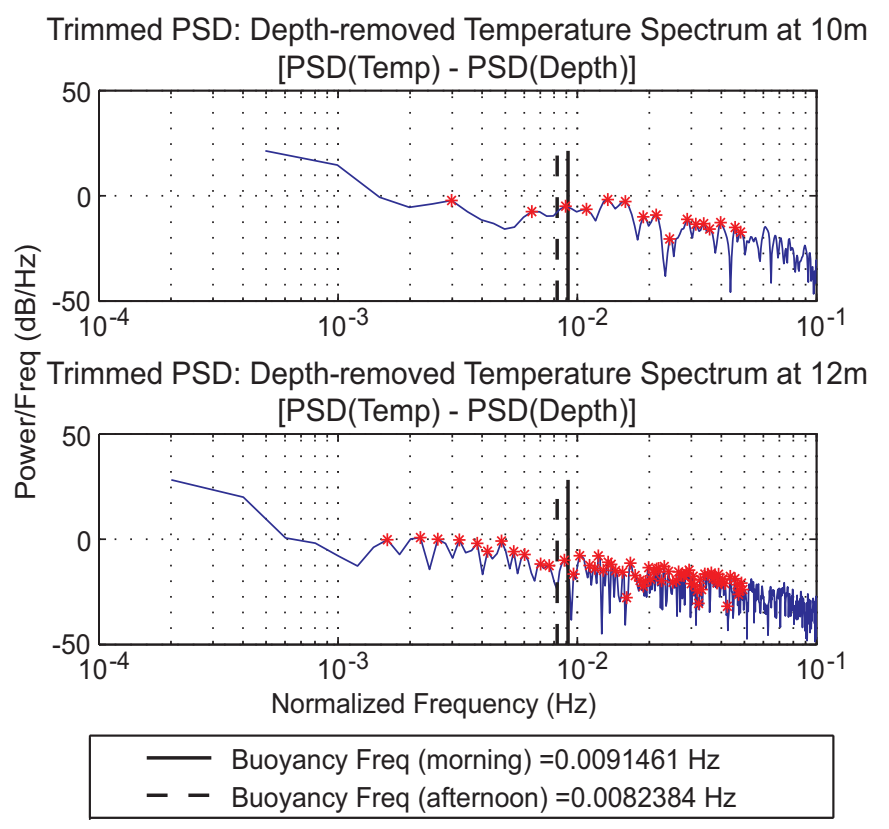

Figure 4-6: Power Spectral Density plot from Unicorn's temperature data (depth variations removed) while traveling at $10 \mathrm{~m}$ (top plot) and $12 \mathrm{~m}$ (bottom plot) depth. Red stars correspond to local peak frequencies in the data spectra.

of this plot, to highlight distinct lower-frequency peaks). Again, this peak is probably due to sensor noise. Other potentially interesting peaks appear below $0.015 \mathrm{~Hz}$ at about 500,1300, 1800, 1900, 2200-2400, $2600,3100,4500,5100,5600,6300$, and 7400 s, some of which may belong to internal soliton waves.

\section{Thermistor Chain}

A set of ten thermistors was deployed attached to the wet cable of the gateway buoy, positioned at the center of the AUV loiter pattern. The thermistors were at depths of 5, 8, 11, 14, 17, 21, 24, 27, and $30 \mathrm{~m}$, with the tenth thermistor placed $0.5 \mathrm{~m}$ above the modem transducer. Since the precise depth of the tenth thermistor was not recorded, and the data set is similar to that of the $30 \mathrm{~m}$ thermistor only flatter (more isothermal) and about $2{ }^{\circ} \mathrm{C}$ cooler, it has been chosen to ignore this thermistor in our analysis. The thermistor chain began recording at 0600 UTC with a sampling frequency of $1 / 30 \mathrm{~Hz}$, and continued to record the temperature through its recovery at about 1415 UTC. The temperature data for the upper 9 thermistors are shown in Fig. 4-10, ordered from shallowest (top) to deepest (bottom), plotted over time. Fluctuations in temperature are most prominently observed in the data from the thermistor at $11 \mathrm{~m}$ depth (closest thermistor to the thermocline depth), which may be indicative of internal waves propagating along the thermocline.

The spectrogram of the $11 \mathrm{~m}$ thermistor's temperature was plotted over varying time spans corresponding 

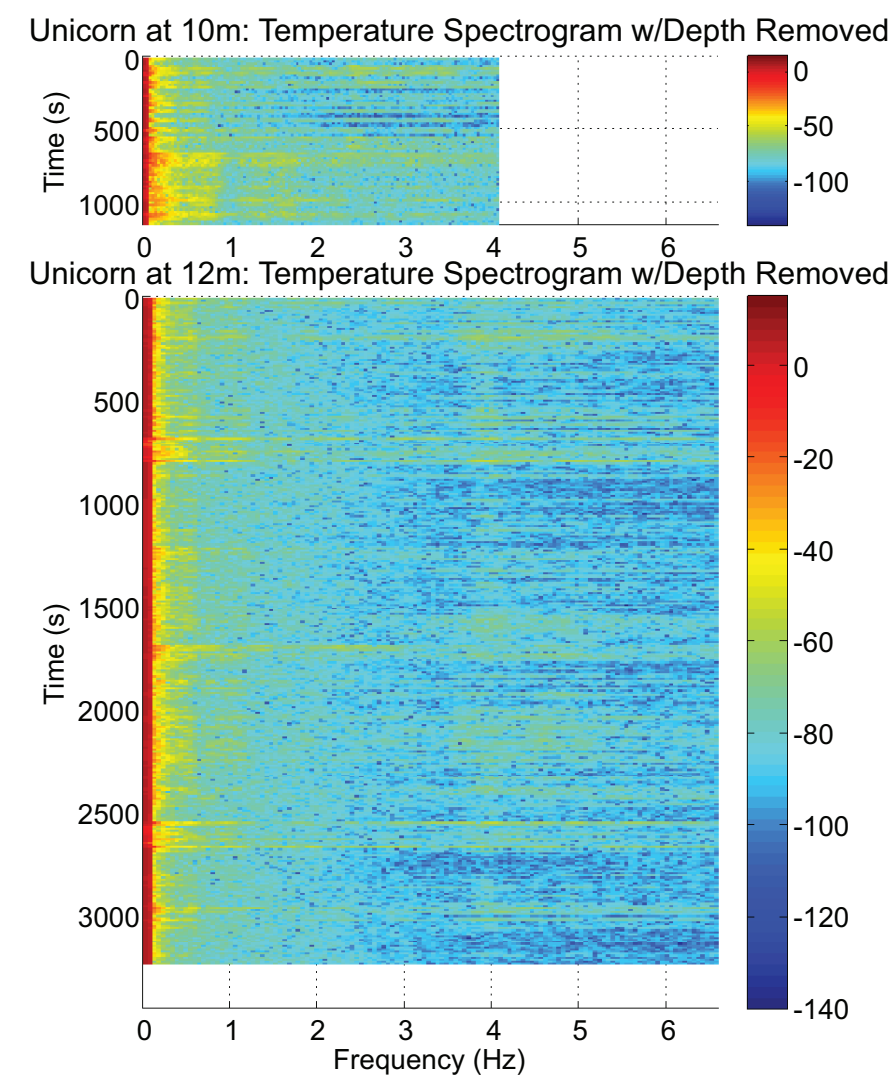

Figure 4-7: Spectrogram of Unicorn's temperature data (depth variations removed) while swimming at $10 \mathrm{~m}$ (top plot) and $12 \mathrm{~m}$ (bottom plot) depth. Hamming window length: 256 samples. Color axis units: dB.

to when Unicorn was swimming at 10 and $12 \mathrm{~m}$ depth and when Harpo was swimming at $12 \mathrm{~m}$ depth (Fig. 4-11). These were visually compared to the spectrograms of the AUV-collected temperature data, and there is general qualitative agreement in times indicating low-frequency peaks, despite differing temporal resolutions. This range of temporal resolutions is due to the difference in sampling frequencies between the AUVs (about $4 \mathrm{~Hz}$ for Harpo and $10 \mathrm{~Hz}$ for Unicorn) and the thermistors $(1 / 30 \mathrm{~Hz})$.

The PSD plots of the temperature data for the $11 \mathrm{~m}$ thermistor are shown in Fig. 4-12, with the peak PSD frequencies plotted as red stars. Here dominant internal wave frequencies are seen between $10^{-3}$ and $10^{-2} \mathrm{~Hz}$ (periods of 17-1.7 min, respectively) in all of the spectra that are similar to peaks in the AUVs' temperature spectra, while the full-length thermistor spectrum (top plot) also shows low-frequency peaks in the $10^{-4}-10^{-3} \mathrm{~Hz}$ range (periods of $170-17 \mathrm{~min}$, respectively). The time-windowed thermistor spectra corresponding to times when the AUVs were at 10 and $12 \mathrm{~m}$ all have dominant frequencies of approximately 


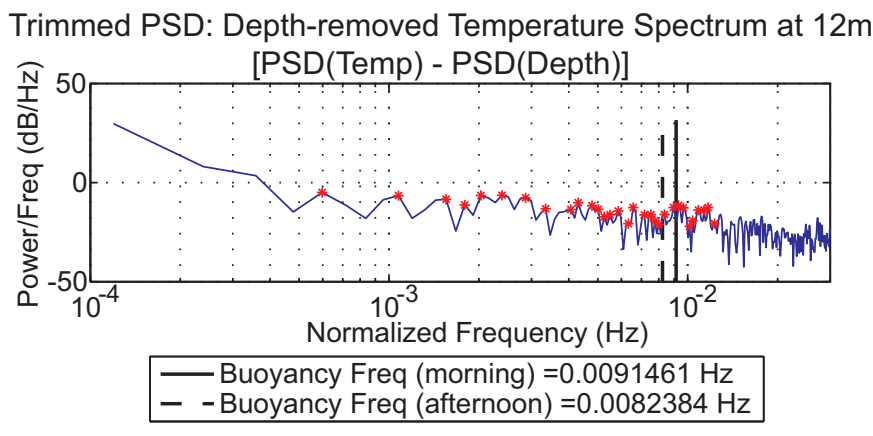

Figure 4-8: Power Spectral Density plot from Harpo's temperature data (depth variations removed) while traveling at $12 \mathrm{~m}$ depth. Red stars correspond to local peak frequencies in the data spectrum.

$2 \times 10^{-3}, 3 \times 10^{-3}$, and $6 \times 10^{-3} \mathrm{~Hz}$ (periods of about 8,6 , and $3 \mathrm{~min}$, respectively), strongly indicative of internal waves.

\section{Buoyancy Frequency Analysis}

It was chosen to first look at buoyancy frequency analysis with the dispersion relation (Equation 4.2) to solve for internal wave wavelength. Buoyancy frequency analysis states that the density difference over the thermocline interface supports its own 'buoyancy' frequency at which the interface is most likely to sustain internal waves [68]. Equation 4.2 approximates the baroclinic or internal mode of the vertical profile of the Tuscan Archipelago basin as a finite layer overlying an infinitely deep layer, with a density discontinuity (thermocline/pycnocline) at the interface between the two layers, giving

$$
\omega^{2}=\frac{g k\left(\rho-\rho_{0}\right) \sinh (k h)}{\rho \cosh (k h)+\rho_{0} \sinh (k h)},
$$

where $\omega$ is the angular frequency in radians $/ \mathrm{s}, g$ is $9.81 \mathrm{~m} / \mathrm{s}^{2}$ (gravitational acceleration), $\rho_{0}$ is the density above the pycnocline, $\rho=\rho_{0}+\rho^{\prime}$ is the density below the pycnocline, $\rho^{\prime}$ is the density change across the pycnocline in the direction of increasing depth, $k$ is the wavenumber in radians $/ \mathrm{m}$, and $h$ is the pycnocline depth in $\mathrm{m}(\sim 11 \mathrm{~m}$, experimentally determined). This form of the dispersion relation also assumes that there is a free surface, which gives rise to a barotropic or surface mode that is beyond the scope of this chapter. See [69] for more details on this form of the dispersion relation.

Given the temperature and density profiles taken the day of the experiment (Fig. 4-3), the thermocline and pycnocline depths were approximated as being equal and these terms are used interchangeably in this section. Starting from Equation 4.3 below (the vertical component of the linearized Boussinesq equations for 


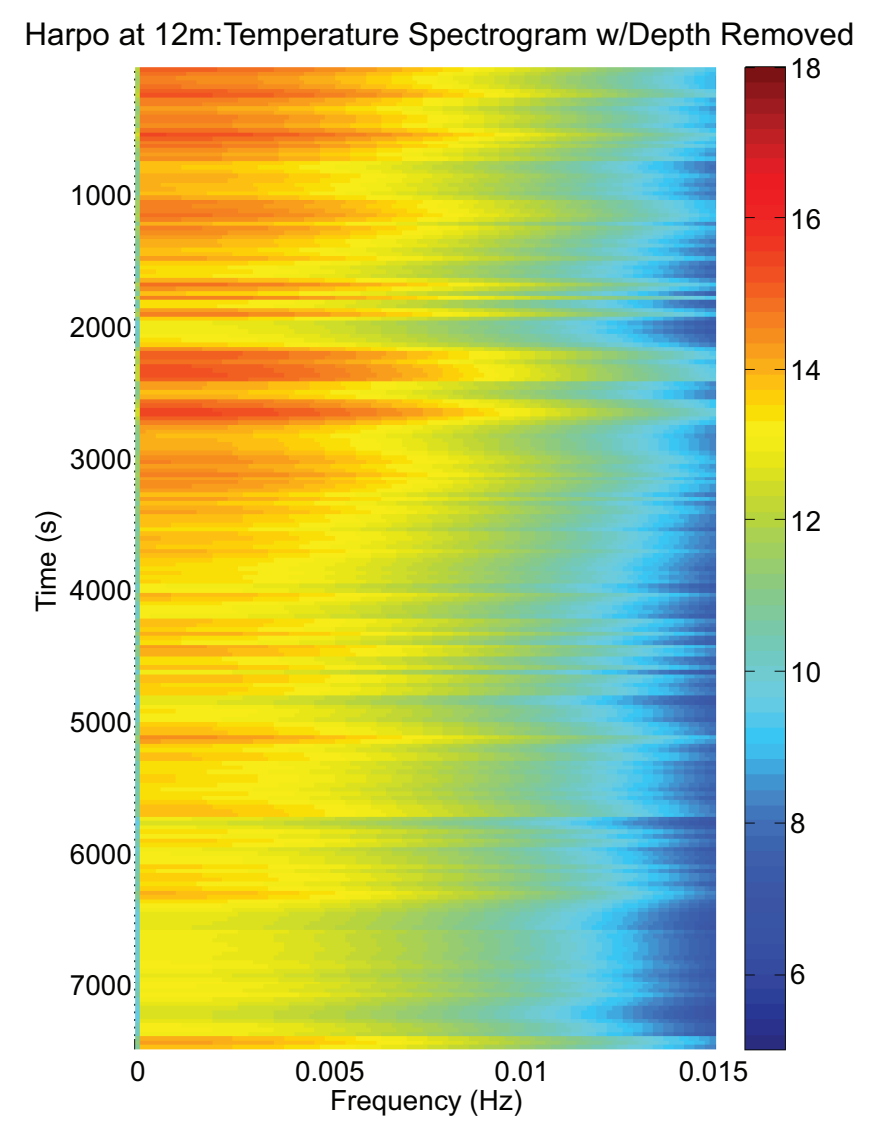

Figure 4-9: Spectrogram of Harpo's temperature data (depth variations removed) while swimming at $12 \mathrm{~m}$ depth. Hamming window length: 256 samples. Color axis units: $d B$.

an inviscid liquid), we can define the Brunt-Väisälä frequency, or buoyancy frequency, $N$ as in Equation 4.4,

$$
\begin{gathered}
\frac{\partial^{2} \eta}{\partial t^{2}}=\frac{g}{\rho} \frac{\partial \rho_{0}}{\partial z} \eta \\
N=\left(-\frac{g}{\rho} \frac{\partial \rho_{0}}{\partial z}\right)^{1 / 2}
\end{gathered}
$$

where $\eta$ is the amplitude of the internal wave, $\rho=\rho_{0}+\rho^{\prime}$ is the density of the fluid layer below the thermocline, and $N(=\omega)$ is an angular frequency of simple harmonic motion. Further details on the physics behind this buoyancy analysis can be found in $[68,69]$.

Using the Unesco 1983 equation of state for sea water [67], density was calculated based on the temperature, salinity, and pressure data collected across a $12 \mathrm{~m}$ depth over the course of the field experiment. 


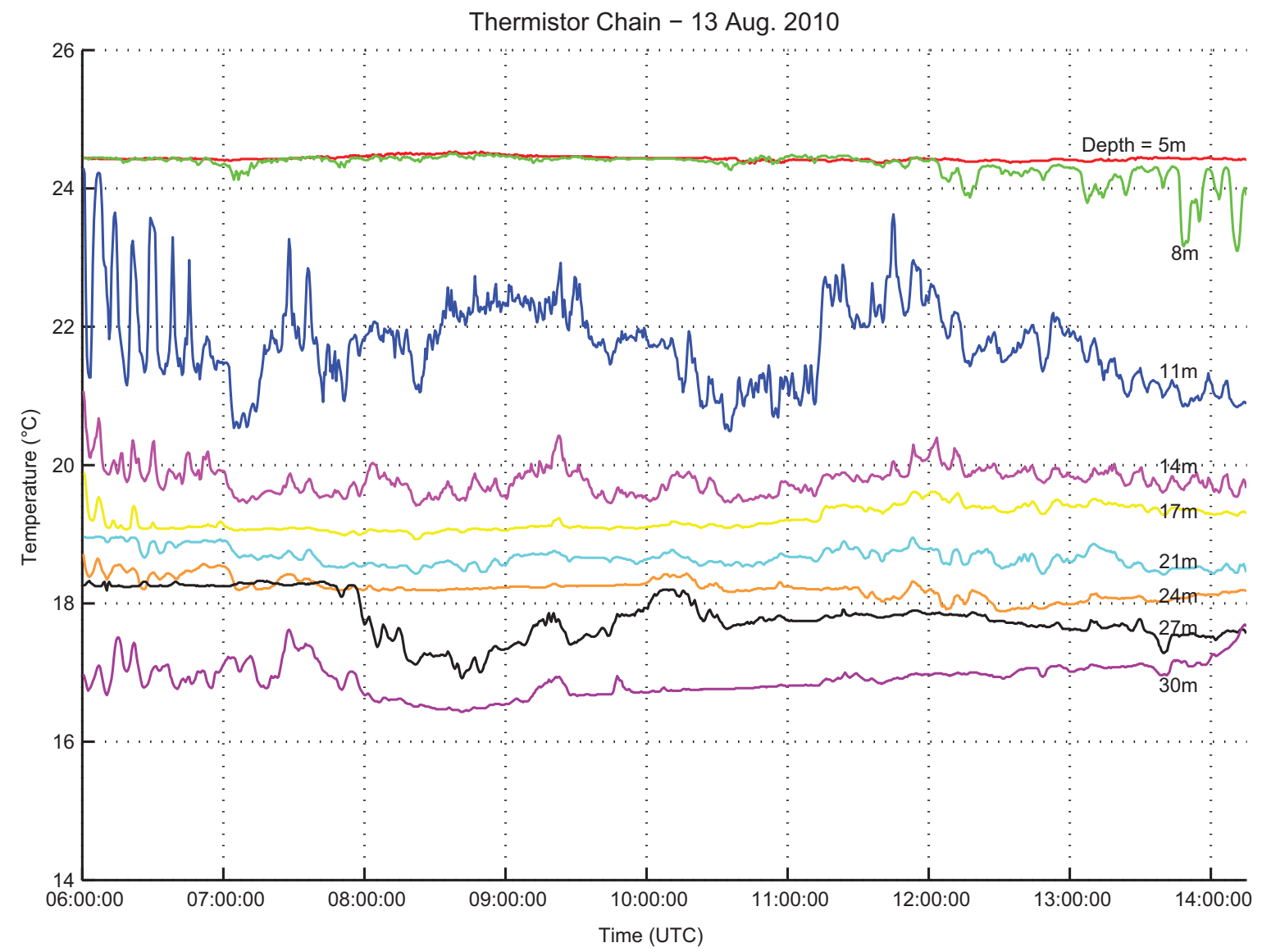

Figure 4-10: Time series of temperature from the upper 9 out of 10 thermistors on the thermistor chain (the deepest thermistor is not shown since its precise depth was unknown). Increased temperature fluctuations are evident at the $11 \mathrm{~m}$ deep thermistor, closest to the $10 \mathrm{~m}$ thermocline depth.

From the density and depth data, the partial derivative in Equation 4.4 is estimated as a finite difference over the pycnocline depth for both the morning and afternoon density profiles and solve for the bounding values of $N_{\text {morning }}=0.05747 \mathrm{rad} / \mathrm{s}$ (linear frequency of $f_{\text {morning }}=0.009146 \mathrm{~Hz}$, period of $T_{\text {morning }}=$ $109.34 \mathrm{sec}$ ) and $N_{\text {afternoon }}=0.05176 \mathrm{rad} / \mathrm{s}$ (linear frequency of $f_{\text {afternoon }}=0.008238 \mathrm{~Hz}$, period of $\left.T_{\text {afternoon }}=121.38 \mathrm{sec}\right)$. Since the CTD cast data that these values are calculated from occurred just before and after the Internal Wave Detection Experiment on August 13, 2010, the calculated buoyancy frequency values can be taken as the upper and lower bounds for that day. The morning and afternoon linear buoyancy frequencies are plotted on the PSD plots in Figs. 4-6, 4-8, and 4-12. According to Kundu and Cohen [69], internal gravity waves are only sustainable below the buoyancy frequency along the interface (pycnocline). Thus, we will disregard all peak frequencies detected above $f_{\text {morning }}=0.009146 \mathrm{~Hz}$. It is evident that there are a number of small peaks near and just below the buoyancy frequency in the AUV and thermistor PSD 


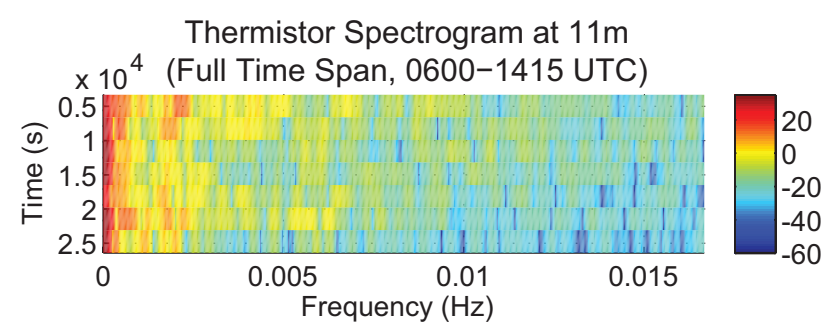

Thermistor Spectrogram at $11 \mathrm{~m}$ w/Unicorn at $10 \mathrm{~m}$ (0833-0853 UTC)

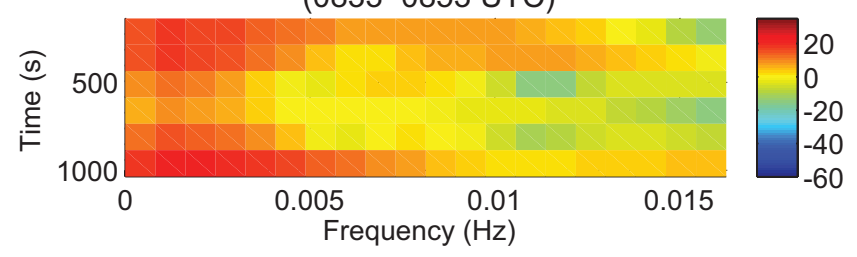

Thermistor Spectrogram at $11 \mathrm{~m}$ w/Unicorn at $12 \mathrm{~m}$ (0913-1007 UTC)

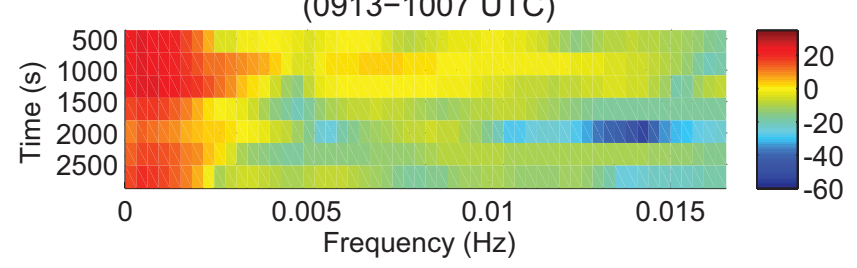

Thermistor Spectrogram at $11 \mathrm{~m}$ w/Harpo at $12 \mathrm{~m}$ (1139-1250 UTC)

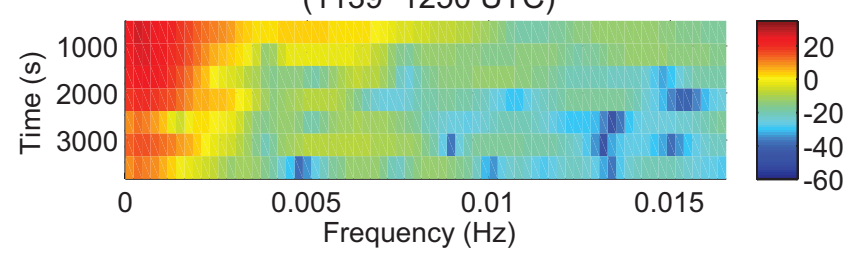

Figure 4-11: Spectrograms of the temperature data from the thermistor at $11 \mathrm{~m}$. From top to bottom: spectrogram of the full time span while the thermistor chain was in the water (Missions 1 and 2), the time span while Unicorn was at $10 \mathrm{~m}$ (Mission 1), the time span while Unicorn was at $12 \mathrm{~m}$ (Mission 1), and the time span while Harpo was at $12 \mathrm{~m}$ (Mission 2). No windowing. Color axis units: $\mathrm{dB}$.

plots, strongly suggesting that buoyancy-supported internal waves propagated through the operation region during the experiment.

The dispersion relation, Equation 4.2, can now be used (with $\omega=N, h_{\text {morning }}=11.79 \mathrm{~m}$, and $\left.h_{\text {afternoon }}=10.39 \mathrm{~m}\right)$ to solve for $k$. Solutions for wavelength $(\lambda)$ and wave phase speed $\left(c_{p}=\lambda f\right)$ follow naturally from Equations 4.5 and 4.6 shown below.

$$
\lambda=\frac{2 \pi}{k}
$$



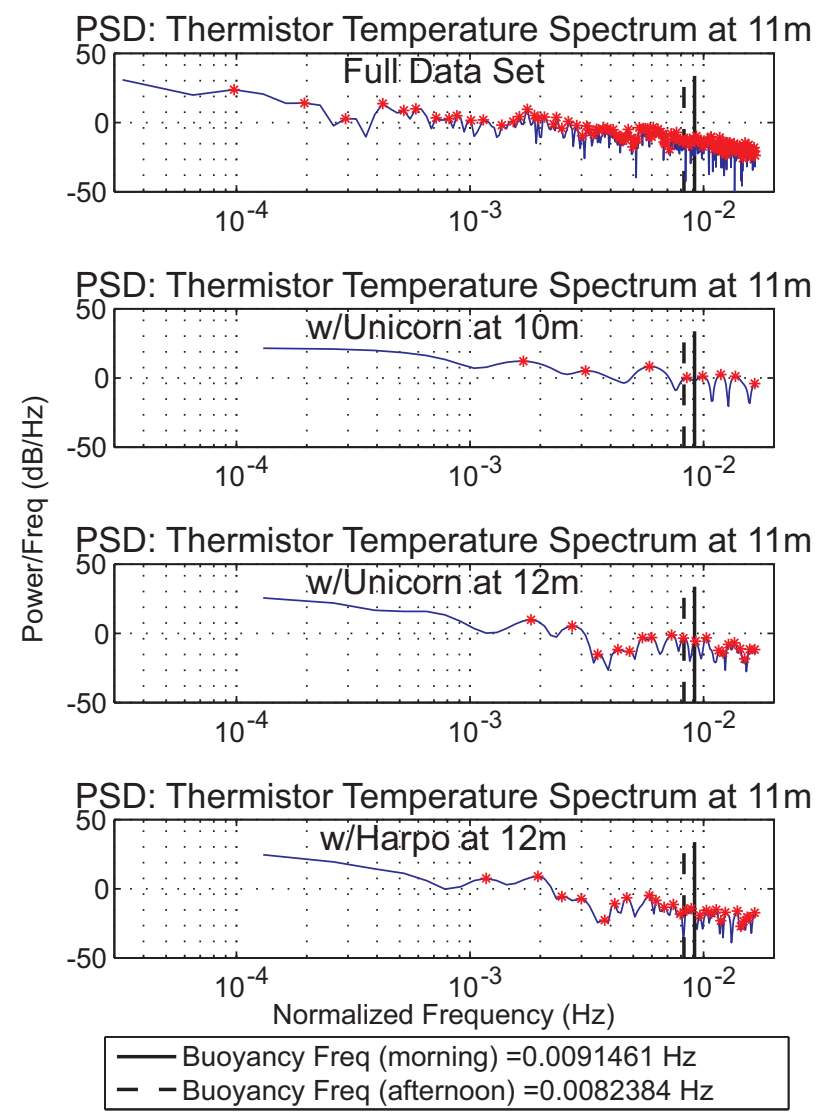

Figure 4-12: PSDs of the temperature data from the thermistor at $11 \mathrm{~m}$. From top to bottom: spectrogram of the full time span while the thermistor chain was in the water (Missions 1 and 2), the time span while Unicorn was at $10 \mathrm{~m}$ (Mission 1), the time span while Unicorn was at $12 \mathrm{~m}$ (Mission 1), and the time span while Harpo was at $12 \mathrm{~m}$ (Mission 2). Red stars are peaks in the spectra.

$$
k=\frac{\omega}{c_{p}}
$$

Using a graphical solution method due to the nonlinear nature of the dispersion relation, the values for $k, \lambda$, and $c_{p}$ of internal waves were estimated as summarized in Table 4.4, where $c_{p}$ is the maximum phase speed. In shallow water, the dispersion relation may often be simplified further by assuming that any waves supported on the interface between two fluids of different density will have wavelengths much longer than the average water depth, $H=150-200 \mathrm{~m}$ (i.e., $\lambda \gg H$ ). However, the shallow-water (long-wave) approximation cannot be assumed here, since $\lambda$ is less than the water depth by an order of magnitude (and on the order of the pycnocline depth), based on the unsimplified dispersion relation in Equation 4.2.

With the maximum phase speed, $c_{p}$, of the buoyancy-driven internal waves calculated to be $0.09315 \mathrm{~m} / \mathrm{s}$, 
Table 4.4: Expected internal wave values calculated using the buoyancy frequency

\begin{tabular}{|l|l|l|l|l|l|l|l|}
\hline $\begin{array}{l}\text { Time } \\
(\mathbf{U T C})\end{array}$ & $N(\mathbf{r a d} / \mathbf{s})$ & $f(\mathbf{H z})$ & $T(\mathbf{s})$ & $h(\mathbf{m})$ & $k(\mathbf{r a d} / \mathbf{m})$ & $c_{p}(\mathbf{m} / \mathbf{s})$ & $\lambda(\mathbf{m})$ \\
\hline \hline $05: 26: 33$ & 0.05747 & 0.009146 & 109.34 & 11.79 & 0.6169 & 0.09315 & 10.18 \\
\hline $13: 55: 15$ & 0.05176 & 0.008238 & 121.38 & 10.39 & 0.6161 & 0.08402 & 10.20 \\
\hline
\end{tabular}

the temperature sensors on both Unicorn and Harpo (and the thermistor chain) had ample time ( $\sim 3$ hrs per wave) to gather enough data to resolve the internal wave motion through the $1.1 \mathrm{~km}$ diameter of the loiter. In the case of Mission 1, Unicorn was at $10 \mathrm{~m}$ for $20 \mathrm{~min}$ and at $12 \mathrm{~m}$ for $54 \mathrm{~min}$, and for Mission 2, Harpo was at $12 \mathrm{~m}$ for $71 \mathrm{~min}$.

Given the very good agreement between the theoretical and data-derived peak wave frequencies (from both the AUVs' and the thermistor's data), along with the slow $\sim 9 \mathrm{~cm} / \mathrm{s}$ phase speed and $\sim 10$ m wavelength of predicted internal waves near the thermocline depth (10-12 m), it is reasonable to conclude that internal waves were positively detected near and below the theoretical buoyancy frequency along the thermocline in the AUV operation region on August 13, 2010, with most frequency components in the $10^{-3}-10^{-2} \mathrm{~Hz}$ range.

\section{Helmholtz-like 'Basin Resonance’ Analysis}

Another possible source of internal waves at the depth of the thermocline may be the Tuscan Archipelago basin acting as a Helmholtz resonator due to flow through the inlets to the basin that lead out to the larger Northern Tyrrhenian Basin (see Fig. 3-12). The canonical example of Helmholtz resonance is the acoustic tone produced by blowing air across the neck of a bottle. The difference in our case is that the restoring force is hydrostatic pressure rather than compressed air, thus it was chosen to call this 'Basin resonance'. As water depth fluctuates with water flowing into and out of the basin, it is possible that a low-frequency wave mode is excited along the thermocline as well. The openings, or inlets, where the forcing of water (and highest flow velocities) into and out of the basin may occur are the numbered segments in Fig. 3-12. The basin inlets are modeled as resonating masses, and the basin body is approximated to be at rest. Equations 4.7 and 4.8 describe this motion as follows:

$$
m_{\text {inlet }} \ddot{x}+\Delta P A_{\text {inlet }}=0,
$$




$$
\Delta P=\frac{\rho g A_{\text {inlet }} x}{A_{\text {surf }}}
$$

where $x$ is the vertical displacement of a fixed-temperature water parcel from the thermocline depth, $m_{\text {inlet }}$ is the mass of an inlet, $A_{\text {inlet }}$ is the cross-sectional area of an inlet, $\Delta P$ is the pressure change due to the basin changing depth, $A_{\text {surf }}$ is the surface area of the basin, $g=9.81 \mathrm{~m} / \mathrm{s}^{2}$ is the gravitational acceleration, and $\rho$ is the average water density. This is analogous to a simple harmonic oscillator described by the differential equation

$$
m \ddot{x}+k x=0,
$$

where $k=k_{e f f}$ is the effective spring constant of the basin given by

$$
k_{\text {eff }}=\frac{\rho g A_{\text {inlet }}^{2}}{A_{\text {surf }}}
$$

The natural (resonant) frequency of a harmonic oscillator is $\omega_{0}^{2}=k / m$. Thus the basin is expected to resonate at

$$
\omega_{0}=\left(\frac{g A_{\text {inlet }}}{A_{\text {surf }} L_{\text {inlet }}}\right)^{1 / 2},
$$

where $L_{\text {inlet }}$ is the length of an inlet. To detect the contributions of different combinations of the five inlets, the ratios, $A_{\text {inlet }} / L_{\text {inlet }}$, of the inlet cross-sectional area to inlet length are averaged over the selected inlets as in Equation 4.12, where the subscript $j \in\{1,2,3,4,5\}$ (some combination of any of the 5 inlets), giving

$$
\omega_{0}=\left(\frac{g}{A_{\text {surf }}}\left(\frac{A_{\text {inlet }_{j}}}{L_{\text {inlet }_{j}}}\right)_{\text {avg }}\right)^{1 / 2} .
$$

If the basin is estimated to cover an area of $A_{\text {surf }}=3880 \mathrm{~km}^{2}$ (estimated using Google Earth [64] in conjunction with a number of Internet-based area calculator tools for KML files) and the the inlet dimensions are as given in Table 4.5, the resulting Basin frequencies are calculated by $f_{B a s i n}=\omega_{0} / 2 \pi$.

Fig. 4-13 summarizes the Basin resonance frequencies compared to the low-frequency peaks in the AUVs' and thermistor's temperature data near the thermocline. Looking at the internal wave frequencies derived from the Unicorn and Harpo data, no evidence of Basin resonance can be seen in the waves along the thermocline. This is not surprising, given that the AUV missions ranged from 20 to 71 minutes in duration, which were 
Table 4.5: Inlet dimensions corresponding to inlets in Fig. 3-12, estimated using Google Earth [64]

\begin{tabular}{|l|l|l|l|l|}
\hline Inlet & Width $(\mathbf{m})$ & Depth $(\mathbf{m})$ & Area, $A\left(\mathbf{m}^{2}\right)$ & Length, $L(\mathbf{m})$ \\
\hline \hline 1 & 7933 & 10 & 79330 & 4309 \\
\hline 2 & 12635 & 40 & 505400 & 2710 \\
\hline 3 & 28944 & 120 & 3473280 & 4700 \\
\hline 4 & 42670 & 450 & 19201500 & 6000 \\
\hline 5 & 13700 & 80 & 1096000 & 9350 \\
\hline
\end{tabular}

barely long enough to span most of the possible Basin resonance periods due to certain inlets. When compared to the frequencies in the $11 \mathrm{~m}$ thermistor data (covering over 8 hours), however, there are a number of lowfrequency peaks in the vicinity of the Basin resonances. Thus, it is very likely that some evidence of Basin resonance is being observed in the thermistor's temperature spectrum at $11 \mathrm{~m}$ depth.

It is important to note that the calculated Basin frequencies in Fig. 4-13 may shift depending on the estimate of the basin surface area. Thus, the thermistor markers tend to align with different, but neighboring, Basin frequencies if the surface area is estimated differently. With an estimated $A_{\text {surf }}=3880 \mathrm{~km}^{2}$, an alignment is observed with the resonant frequency imparted by inlets 2, 3, and 4 combined (see Fig. 4-13). This alignment is not surprising, given that inlets 2,3 , and 4 are the widest inlets and are the inlets most exposed to flows through deep channels outside the Tuscan Archipelago basin. This implies that inlets 2, 3, and 4 would be the most likely combined driving force for Basin resonance, and this is substantiated by the data.

\subsection{Receiver Operating Characteristic Curves}

Receiver operating characteristic (ROC) curves may be applied to the thermocline tracking data from the Internal Wave Detection Experiment as a measure of thermocline tracking performance. These curves compare the probability that the AUV performing thermocline tracking will correctly detect and track the thermocline location (probability of detection, $P_{D}$ ) to the probability that it will falsely detect and track the thermocline (probability of false alarm, $P_{F A}$ ), for a variety of threshold values. The ROC curve for thermocline tracking is generated based on the probability density functions (PDFs) of the magnitudes of the temperature gradients $(|\partial T / \partial z|)$ in vertical space at the thermocline while thermocline tracking (signal plus noise) and away from the thermocline (noise), at a depth of $60 \mathrm{~m}$. From these PDFs, the $P_{D}$ and $P_{F A}$ can be determined as the area under the curves for the within-thermocline and away-from-thermocline AUVs, respectively, to the right of 


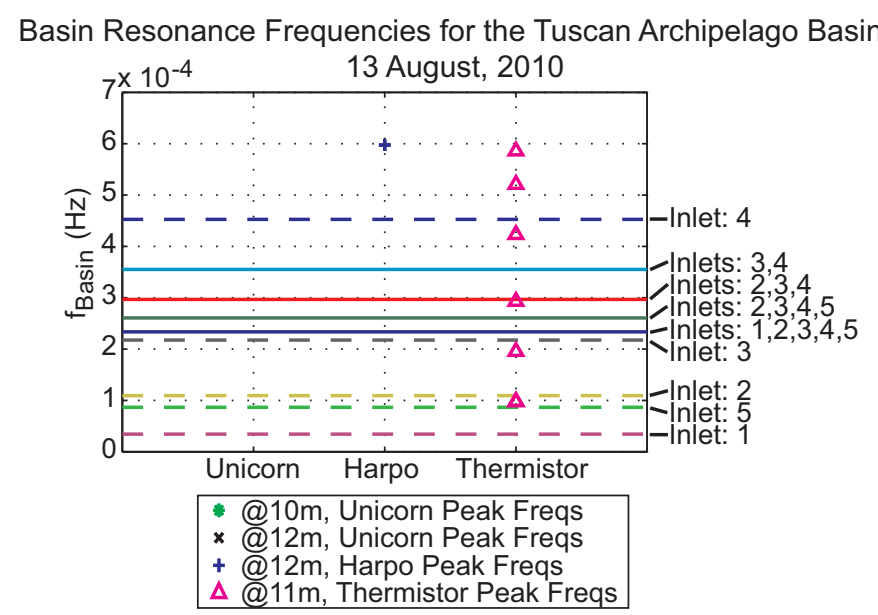

Figure 4-13: Basin resonance frequencies, compared to peak frequencies from the AUVs' and thermistor's temperature spectra near the thermocline. The lines represent the Basin resonant frequencies of the Tuscan Archipelago basin based on the estimated basin surface area of $A_{\text {surf }}=3880 \mathrm{~km}^{2}$. Each line accounts for a different subset of inlets to the basin in order to determine which inlets play a dominant role in the Basin resonance. The shaped markers highlight the temperature spectra peak frequencies in the Basin resonance range detected in the Unicorn, Harpo, and thermistor thermocline data. Note that Unicorn detected no frequencies near the Basin resonance. Also note that the calculated Basin frequencies may shift depending on the estimate of the basin surface area. Thus, the thermistor markers tend to align with different neighboring Basin frequencies if the surface area is estimated differently.

a threshold temperature gradient value. The threshold value is selected based on the temperature change over each yo-yo leg and on the yo-yo amplitude. Fig. 4-14 plots the resulting ROC curve for thermocline tracking. This suggests that the thermocline tracking method used here can have a decent probability of detection $(80 \%)$ with a reasonably small probability of false alarm $(23 \%)$.

\subsection{Conclusion}

This chapter is centered around the Internal Wave Detection Experiment using AUVs in the Tuscan Archipelago basin that took place on August 13, 2010. Experiment design, hardware and code for implementation, resulting field trials, and post-deployment data results and analysis are discussed. This experiment took a novel approach to internal wave detection by tasking two autonomously collaborating AUVs to autonomously adapt their motion in relation to each other and to their dynamic environment, resulting in greater efficiency of sampling given a restrictive mission duration and in collection of fully synoptic data sets capturing internal waves.

The Internal Wave Detection Experiment involved two AUVs running the MOOS autonomy system 


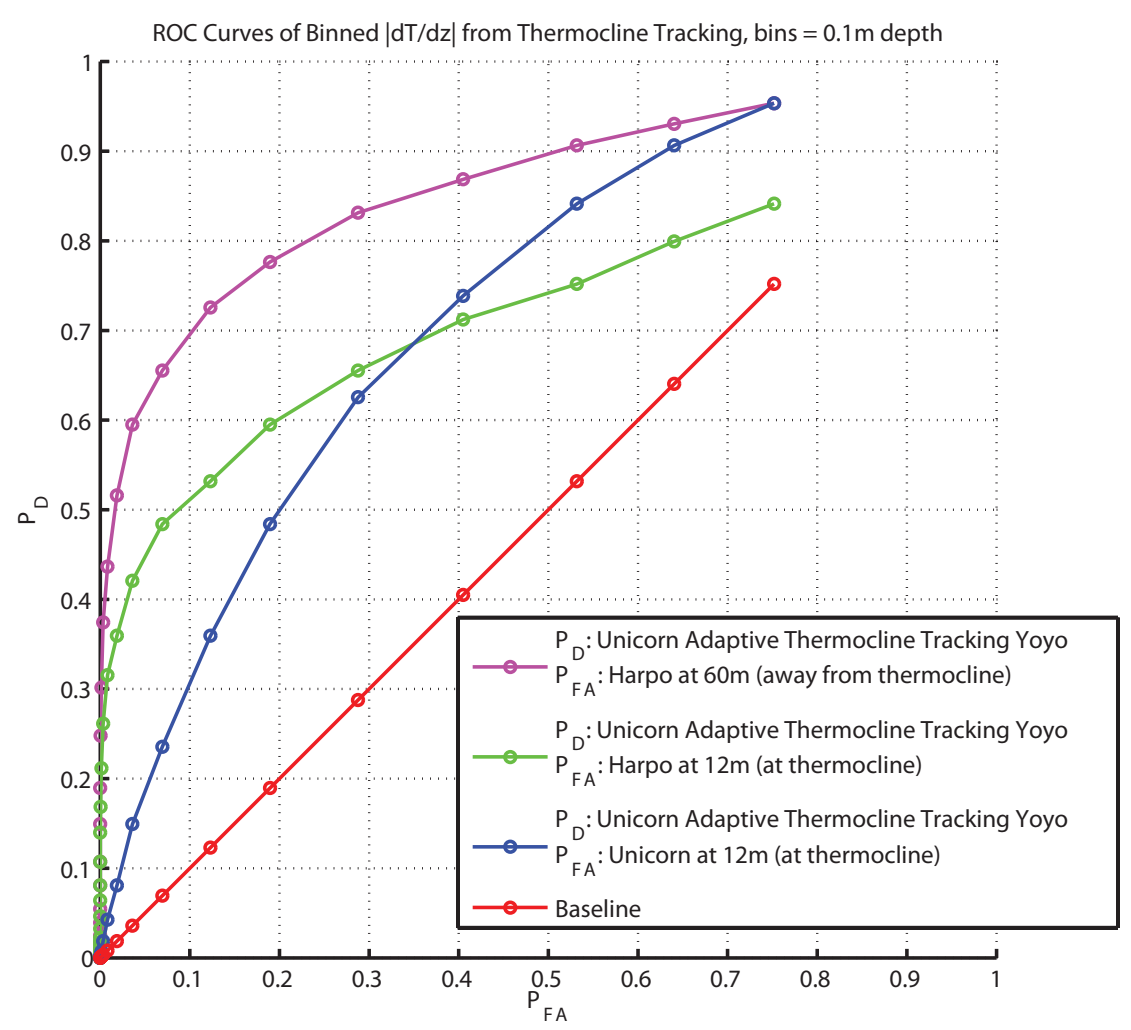

Figure 4-14: ROC curves of the performance of the adaptive thermocline tracking method from Chapter 3 used during the Internal Wave Detection Experiment. The magenta curve compares the $|\partial T / \partial z|$ thermocline tracking signal to swimming at a constant depth of $60 \mathrm{~m}$, which is well below the depths where a thermocline signal is present. This provides a clear performance evaluation of thermocline tracking by comparing the thermocline tracking signal plus noise to the thermal noise down at $60 \mathrm{~m}$ depth. The blue and green curves compare thermocline tracking to swimming at $12 \mathrm{~m}$ depth, just below the thermocline. As expected, it is harder to positively detect the thermocline due to an increase in false alarms where there is greater background noise through fluctuations in temperature. The red curve is a baseline for comparison, where the probability of detection, $P_{D}$, is equal to the probability of false alarm, $P_{F A}$. Curves following the left and top edges of the plot exhibit 'better' performance.

guided by the IvP Helm. These AUVs used acoustic communication during the experiment to send and receive real-time data and status updates, which they used to autonomously coordinate their motions in the horizontal plane through a track-and-trail behavior. In the vertical axis, the Unicorn AUV autonomously adapted to changes in the environment while the Harpo AUV (which would have also adapted if the thermocline depth allowed for more reliable acoustic communication) swam just below the thermocline. A thermistor chain was also deployed for the duration of the experiment.

In examining the resulting AUV and thermistor data sets from this experiment, there is strong evidence of internal wave propagation along the thermocline near the buoyancy frequency of the thermocline interface $\left(N_{\max }=0.05747 \mathrm{rad} / \mathrm{s}\right)$. Internal waves with nearly identical and lower frequencies were seen in the 
Unicorn, Harpo, and thermistor data collected near the thermocline depth. The $12 \mathrm{~m}$ AUV and $11 \mathrm{~m}$ thermistor results suggest the presence of buoyancy-supported internal waves along the thermocline (about $11 \mathrm{~m}$ depth) in the AUV operation region throughout the day on August 13, 2010. This conclusion may also be extrapolated to say that internal waves are likely detectable along the thermocline throughout the rest of the Tuscan Archipelago basin during the summer, when the thermocline is fairly well defined. Given the lack of previous literature regarding internal waves in the Tuscan Archipelago basin, this finding is rather significant to the scientific groups that conduct acoustic (and other) experiments in this region.

Internal waves due to Basin resonance (a concept similar to Helmholtz resonance) in the basin were also examined. The results suggest that both single inlets and combinations of inlets (see Fig. 3-12 and 413) excite internal wave frequencies within the basin that are detectable by a thermistor chain (and AUVs) deployed for long (multi-hour) missions. However, it is likely that inlets 2, 3, and 4 combined contribute a stronger resonance to the internal waves in the basin due to deep topography and currents just outside these basin inlets. This point is reinforced by the close alignment of one thermistor-detected frequency with the Basin resonance frequency from inlets 2, 3, and 4 combined. This supports the theory of the presence of low-frequency internal waves due to Basin resonance in the Tuscan Archipelago basin.

An ROC (Receiver Operating Characteristic) curve performance analysis of the thermocline tracking method employed in the Internal Wave Detection Experiment suggests that this thermocline tracking method can have a decent probability of thermocline detection and tracking $(80 \%)$ with a reasonably small probability of false alarm (23\%). Although this is not ideal, it is very reasonable given the dynamic environmental conditions.

Overall, this experiment was novel in the internal waves data set it captured in the Tuscan Archipelago Basin and its use of multiple AUVs collaborating autonomously with each other and autonomously collecting environmentally-adaptive data sets for more synoptic spatio-temporal data coverage. Not only does this increase the efficiency of data collection (environmentally-adaptive autonomy behaviors allow collection of the exact data set needed without a human in the loop), but also the ability to collect the specific data set of interest to the researcher by using AUVs running autonomy. The use of intelligent acoustic communication networking also allows the AUV operators and scientists to monitor (from the topside on a ship or shore) the data collected in near real time. These abilities are invaluable when ship time for data collection is expensive, and provides encouragement that improvements in AUV autonomy, adaptive environmental sampling techniques, and acoustic communications will enable a further reduction in necessary ship time for scientists and engineers to collect the specific data sets required in the future. 


\subsection{Looking Ahead}

\subsubsection{Further Data Analysis \& Future Experiments}

Future work relating to this data set could include attempting to tease out the general direction of internal wave propagation from the AUVs' temperature data when divided into the five separate headings (one for each leg of the pentagonal loiter). If the peak frequencies of the temperature spectra increase or decrease slightly as the heading changes, the highest observed frequencies will correspond to the AUV heading nearly opposite of the direction of internal wave propagation, and the lowest observed frequencies will correspond to the AUV heading nearly perpendicular to the direction of internal wave propagation. If the phase speed of the internal waves (propagating as a soliton or a larger group of waves) is on the order of the speed of the AUVs or less, as seen in this experiment, the AUVs most likely intersected the internal waves enough times at each of the 5 headings to be able to solve this problem. However, this may prove an unsolvable challenge in the case of swiftly $(>10 \mathrm{~m} / \mathrm{s})$ propagating internal wave solitons, since solitons would only appear for brief times in the AUV data.

In addition, internal wave amplitude may be estimated by examination of the depth variation of isotherms, particularly those concentrated near the thermocline depth in shallow water. As an internal wave passes a given point in the horizontal plane, the isotherms near the thermocline will rise or drop in depth by some distance indicative of the amplitude of the internal wave. To collect a proper data set for such a measurement, an AUV must collect temperature data in the depth range around the thermocline as the internal waves pass by. This is done (using the autonomy setup) by employing the adaptive thermocline tracking behavior as Unicorn did in Mission 2 of the Internal Wave Detection Experiment. This, however, is beyond the scope of the work presented in this thesis.

It would also be ideal to have another opportunity to execute all three missions of the Internal Wave Detection Experiment, again with at least two AUVs. It would be beneficial to collect environmental data sets for all three missions similar to those already collected, but over longer missions such that multiple hours or days worth of environmental data could be examined for persistent and/or longer period internal waves (including any tidal effects). Also, the goal of Mission 3 was to have the AUVs coordinated in motion (particularly coordinated in heading) through autonomous collaboration but spatially distributed in the horizontal plane such that internal wave speed could be directly estimated from the time it takes a wave crest to pass between the two AUVs on the same heading. Further analysis of data from Missions 1 and 2 in this experiment may reveal similar results for the sections of each loiter leg in which both Unicorn and Harpo have the same heading. 
Finally, it would be important to quantify the hysteresis between the temperature and pressure sensors on Unicorn while yo-yoing. At the very least, a corrective adjustment should be made in the future to the resulting data sets. This will include accounting for the position difference between the CT sensor in the center section of Unicorn and the pressure sensor in its aft section (about $1.5 \mathrm{~m}$ away) and matching Unicorn's temperature values as it passed through the $12 \mathrm{~m}$ depth (during Mission 2) to those of Harpo at $12 \mathrm{~m}$ (also accounting for the fact that Unicorn was about $150 \mathrm{~m}$ behind Harpo).

\subsubsection{Broader Applications}

This chapter has proven that multi-AUV collaboration and adaptive autonomy techniques may be employed to quickly and efficiently detect features such as internal waves within a relatively small operation region. The next step in the process of developing autonomous and adaptive environmental sampling techniques for AUVs would be the process of designing and writing IvP Helm autonomy behaviors that queue off of changes in the environment to guide AUVs (solo or multiple) in actively tracking mesoscale and larger features over 3D space and time. To this end, we have developed 2D (horizontal plane) and 3D front tracking behaviors that allow an AUV to track along the boundary of a oceanographic front. These AUV behaviors, which are discussed in detail in Chapter 5, have been tested in virtual experiments using a 4D MSEAS dynamic ocean model environment and, in some cases, coupled with a follow-the-leader behavior designed for coordinated front tracking using multiple AUVs for improved sampling coverage. 


\section{Chapter 5}

\section{Front Tracking}

\subsection{Introduction}

Oceanic fronts, similar to atmospheric fronts, occur at the interface of two fluid (water) masses of varying characteristics (e.g., temperature, salinity, density, and/or currents). These fronts often also occur in regions of rapidly changing bathymetry, such as coastal shelfbreaks, where water from the deep ocean comes in contact with coastal waters. At these frontal interfaces there may be increases in biological activity, interesting flow patterns, convergence zones where pollutants gather, or other water property variations $[43,44]$. In particular, the meeting of two water masses at a front is an important region to study, as the difference in density between the two water masses result in vertical velocities that cause nutrients to be cycled up from deep in the ocean. This nutrient upwelling plays a critical role in supporting biological productivity near the ocean's surface. Where there are such quantifiable physical, chemical, or biological changes in the ocean environment, it is possible—with the proper instrumentation— to track, or map, the front boundary.

In the case of a front boundary defined primarily by a locally high temperature or salinity gradient, it is possible to use a conductivity-temperature-depth (CTD) sensor to sample the front. CTD sensors can be compact enough to mount on board AUVs and other small oceanographic platforms. Past methods for sampling along and across ocean fronts have included shipboard sampling transects, moored arrays of instruments, and remote sensing via satellites. Only recently have various robotic marine platforms been employed for this purpose. Each of these methods has benefits and drawbacks in terms of sampling resolution and efficiency, synopticity across a range of spatiotemporal scales, and resources necessary to perform sampling surveys. As described by He et al. [43], the field is moving toward employing new AUV fleets for more synoptic and persistent monitoring of certain U.S. coastal regions, such as at the Pioneer Array south of Cape Cod, 
MA, but the infrastructure has yet to be completed. In addition, environmentally adaptive autonomous sampling methods for the AUVs to be deployed at the Poineer Array and similar coastal nodes are not currently being considered for this application due to the increased computational and technological complexity over preplanned transects. As a result, environmentally adaptive autonomous sampling methods are still in the development and testing phases for the smaller AUV groups such as ours that are working on the problem. The approach and method employed for this work is described in this chapter.

\subsection{Goals}

In this chapter, a couple of novel methods for environmentally adaptive autonomous sampling and tracking along an ocean front are proposed and implemented using AUVs by employing the AAEA method described in Chapter 3. The vehicles used for this work run the MOOS and IvP Helm autonomy software on board, including numerous autonomy behaviors that control the AUVs'safety, maneuvering, and sampling paths. A spatiotemporally dynamic MIT MSEAS (Multidisciplinary Simulation, Estimation, and Assimilation Systems) model of the Mid-Atlantic Bight (MAB) region off the east coast of the U.S. is used as a testing environment for virtual experiments, allowing the evaluation of these new AUV front tracking methods.

The results from numerous AUV front tracking virtual experiments (2D) at constant depth are presented, including performance metrics comparing the adaptive front tracking to preplanned survey methods. A couple of behaviors for coordinating multiple AUVs performing 2D front tracking (to retain synopticity and increase spatial coverage) are explored, as well as a behavior for solo AUVs to perform 3D front tracking (to sample the front in the depth dimension, as well as the horizontal plane), and initial results from virtual experiments are presented.

The specific goals here are to apply AAEA and Feature Tracking to adaptively sample along and across an ocean front using only the data collected on board $A U V$ s, gathering a synoptic data set of the position of the front over time while improving sampling efficiency and density over current preplanned AUV sampling surveys.

\subsection{Literature}

The need for AUV front sampling methods is specifically motivated by He et al. [43, 44], where they note that shelfbreak environments in particular, such as the MAB, are difficult to study with the currently used older methods due to their highly dynamic spatiotemporal characteristics. Older methods of front detection, observations, and sampling have included satellite remote sensing looking at characteristics such as sea surface 
temperature [84-86] and shipboard sampling [87-89]. More recently, the use of AUVs has begun to emerge as a leading technology for front sampling. Gottlieb et al. have developed a Momentum-Based Front Detection algorithm for use on AUVs [90], while Reed \& Hover are working on a system for a network of AUVs to track a front by distributing multiple AUVs at fixed points along a front line and having them follow short acrossfront transects back and forth as the front line fluctuates, modeling it as a coupled linear time-invariant (LTI) system [91]. Cannell \& Stilwell present AUV methods for static front and plume mapping using a fixedpath parametric mapping technique and a non-parametric boundary tracking technique that first classifies the entire feature's boundary with a probability distribution and then plans the AUV's path to zigzag along the predetermined boundary [92]. Finally, Zhang et al. have used AUVs to autonomously and adaptively detect and track a coastal upwelling front in Monterey Bay, CA, by defining the frontal boundary based on the horizontal gradient of the vertical temperature distance between deep and shallow depths $[93,94]$. The primary difference between the approach of Zhang et al. and that described in this chapter is that the AUV's tracking of the coastal upwelling front was directly across-front at a single location along the front (including depth profiles), with no along-front component.

\subsection{Novel Concepts \& Approach}

The approach to front tracking developed here is a novel combination of the real-time adaptive autonomy approach presented by Zhang et al. $[93,94]$ and the along-front zigzag method presented by Cannell \& Stilwell [92], resulting in two primary autonomous and adaptive front tracking methods: 2D front boundary tracking with a zigzag pattern and 3D front interface tracking with a horizontal helix pattern. Techniques and autonomy behaviors for multi-AUV front tracking are also addressed, where the method developed in this work encourages travel in the along-front direction as well as across-front mapping.

Following the aforementioned goals, the front tracking methods proposed here emphasize reduced algorithm and implementation complexity to improve robustness for deployment in field experiments in the foreseeable future. In this case, temperature changes are used as the frontal indicator due to the measurement stability and physical size of temperature sensors available for small sub-sea platforms. Temperature, unlike density, can be measured directly, and many small salinity (conductivity) sensors are sensitive to temperature changes, thus making temperature the more robust characteristic to measure.

The front tracking behaviors described in this chapter focus on tracing the front boundary with one or more AUVs in either 2D (constant depth) or 3D space. The underlying behavior for this employs an initial survey of the area followed by a zigzagging motion (in the horizontal plane) back and forth across an isotherm, 
where the isotherm is detected and selected by the AUV as the temperature of the front boundary. As the AUV is constantly collecting temperature and position data, it constantly updates the frontal isotherm temperature and the estimate of the local front position. With these continual updates, the AUV is able to adapt its motion to track the front locally, synoptically sampling along the front and maintaining coverage across the front, even as the front moves in space and time.

The single-AUV 2D (zigzag) front tracking can be directly extrapolated into 3D as a horizontal helix behavior, where the long axis of the helix is at a constant depth and aligned in the horizontal plane with the local front line estimate (see Fig. 5-1), as in the 2D case.

3D Front Tracking: Helix

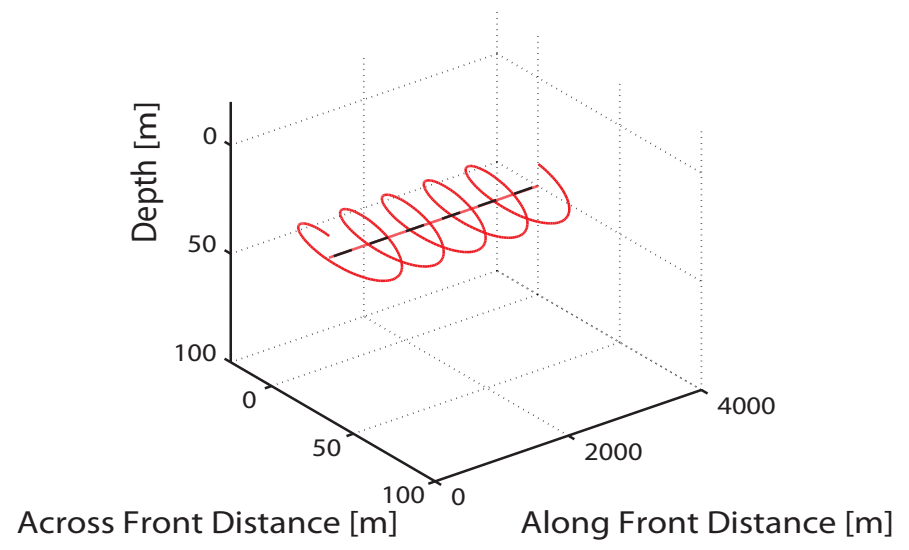

Figure 5-1: A conceptual sketch of the horizontal helix pattern used for 3D front tracking. The helix's center axis is at a fixed depth, aligned with the locally estimated front boundary line (dashed straight line) in the horizontal plane.

To increase spatial coverage in the (horizontal) along-front direction, multiple AUVs may be employed in a follow-the-leader fashion. In this chapter, this multi-AUV method is only coupled with the single-AUV 2D zigzag method (Fig. 5-2) in virtual experiments, but it could also be coupled with the single-AUV 3D helix method mentioned above.

To extrapolate to a multi-AUV mission in $3 \mathrm{D}$, either the $2 \mathrm{D}$ zigzag or $3 \mathrm{D}$ helix can be performed simultaneously (but separately) by all AUVs, where each AUV is assigned a different (central) depth to track at and all AUVs are roughly aligned in a vertical line. 3D multi-AUV missions are beyond the scope of this thesis, but conceptual sketches are shown in Figs. 5-3 and 5-4.

Other front tracking approaches described in related literature range from theoretical simulations with AUVs to determine variation of a front's position assuming a known environment to distributing underwater gliders within the frontal boundary of a plume, as previously discussed in Section 5.3. The simplicity of our 


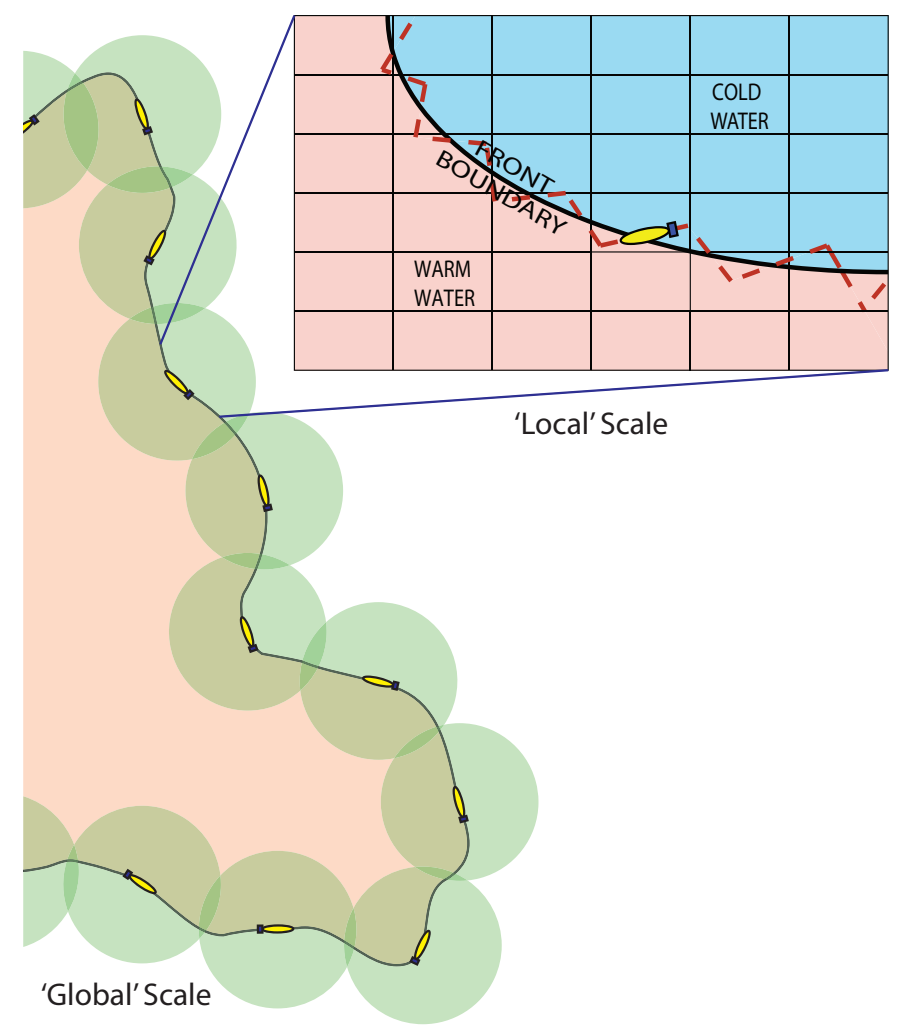

Figure 5-2: A conceptual illustration of 2D multi-AUV front tracking, exhibiting 'global' adaptive follow-theleader motion of AUVs along the front and 'local' 2D adaptive zigzag motion of AUVs across the front. The green circles represent the front's spatiotemporal scales as a distance between AUVs. When these AUV range circles overlap along a front line, the sampling may be considered synoptic. Used with permission from [5].

approach — the zigzag motion and the tracking of an isotherm rather than a temperature gradient (which may dissipate or change from one stretch of the front to another)—-keeps the complexity of this autonomous and adaptive front tracking method to a minimum, which is important for reducing the possible failure modes when deploying this technology in real, dynamic ocean environments. It is also assumed that, due to computational and power limitations on real AUVs and the very limited data transfer available via acoustic communications, the AUVs will have no outside knowledge (e.g., no satellite data and no ocean models uploaded or generated on board) of the environment other than what they collect with their on-board sensors in real time. Thus, the sampling patterns the AUVs decide upon autonomously must yield enough environmental information for them to make informed decisions about where to go next to properly sample the front.

The primary drawbacks to this front tracking method reside in the cases where the AUV 'loses' the primary frontal isotherm, either 1) by the front advecting away from the AUV faster than the AUV can move or 2) by the AUV becoming stuck along a local pocket of isotherm that is greater than $\mathrm{O}(1 \mathrm{~km})$ in horizontal extent 


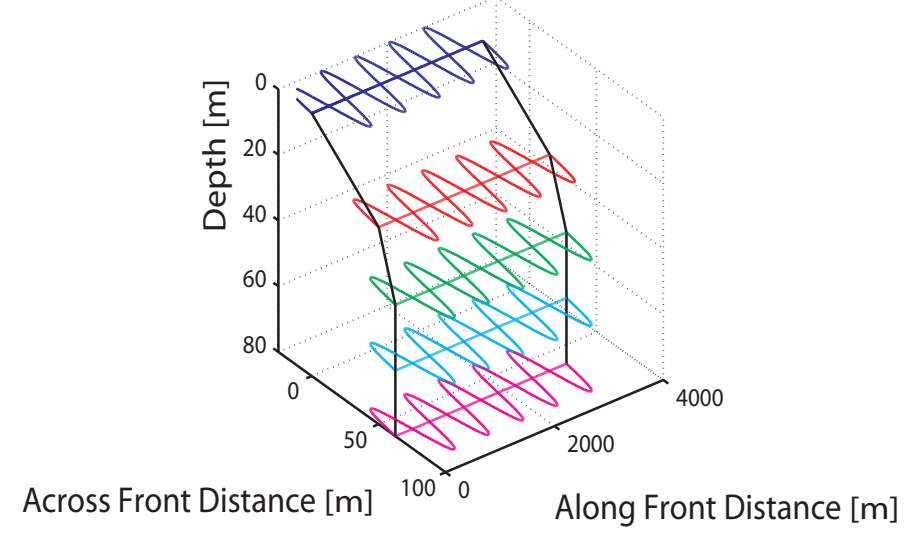

Figure 5-3: A conceptual sketch of a depth-distributed (3D) multi-AUV mission using the 2D AUV zigzag pattern for front tracking. Each color path represents the path of a different AUV, where the AUVs are aligned roughly vertically along the front at a range of depths. The black vertical curve represents a typical stratified temperature profile.

(having the same temperature as the front) but isn't along the primary front. The former of these cases is unavoidable—resulting from the propulsion limitations of the AUV being used—and, thus, is a problem for most front tracking methods. The latter is difficult to avoid whether tracking a front boundary based on an isotherm or an across-front gradient value, since local pockets of the temperature and temperature gradient values may occur on scales ranging up to the mesoscale, making them hard to distinguish from the primary front line when all that is available are point measurements of temperature values that are assumed to be connected into a line if they share the same isothermal or gradient value with the front. In order to reduce time the AUV spends 'lost' and account for the significant spatiotemporal variation of temperature along the front, a timeout is included in the front tracking behaviors that restarts the front tracking process, determining a new frontal isotherm to track near the AUV's location. If an AUV is stuck in a local mesoscale temperature pocket, however, it may still re-find and remain in that pocket after a timeout. This is simply a shortcoming of front tracking methods such as this, where the AUVs determine and track the front location based on a locally sub-mesoscale sampling pattern.

Further details of the implementation and algorithms for the front tracking methods described in this section are provided in Sections 5.5 and 5.6. 


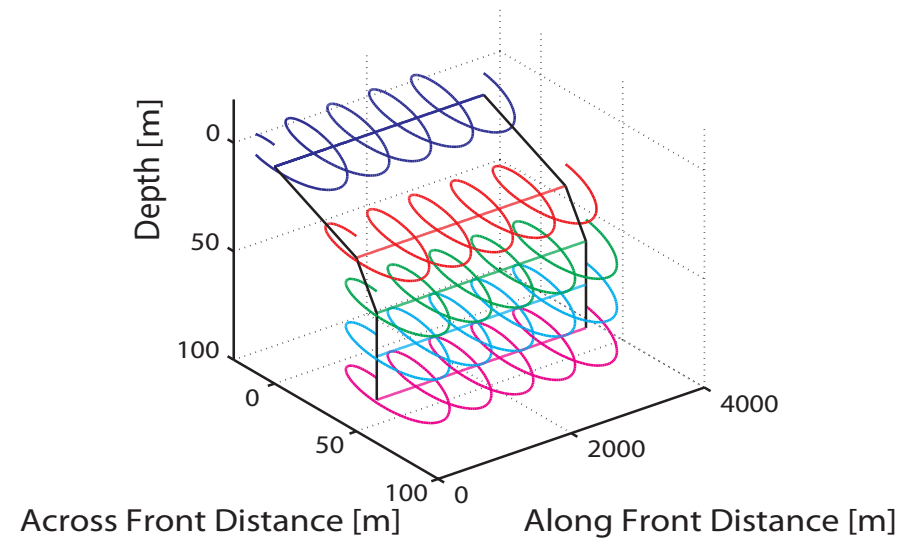

Figure 5-4: A conceptual sketch of a depth-distributed (3D) multi-AUV mission using the 3D horizontal helix pattern for front tracking. Each color path represents the path of a different AUV, where the AUVs are aligned roughly vertically along the front at a range of depths. The black vertical curve represents a typical stratified temperature profile.

\subsection{D \& Multi-AUV Front Tracking}

\subsubsection{Overview}

The basic, 2D (constant depth) front tracking method developed here has three phases: detection, classification, and tracking. This is sketched out in Fig. 5-5. Throughout the front tracking exercise, the AUV is constantly monitoring the water temperature and updating the maximum and minimum temperature values it has encountered. In this case, the front is defined as the isotherm with the temperature half way between the max and min temperatures. A more elegant (but more complex and less robust) approach would be to keep track of spatial temperature gradients and use the maximum temperature gradients to define the front boundary.

\section{Detection}

The AUV performs a survey of the local region to detect the front. This is either a preplanned loiter pattern around a selected center point in space, or a spiral out from the center point. As the AUV travels along its loiter or spiral path, it is actively keeping track of the maximum and minimum temperatures it has encountered. The frontal isotherm is defined as the temperature that is half way between the maximum and minimum recorded temperatures (and, at first, is frequently updating as the AUV follows the first loop around the loiter or spiral). A 'crossing' is recorded, along with its time and location, each time the AUV moves from warmer to colder (or colder to warmer) water across the frontal isotherm. The detection survey continues until three 


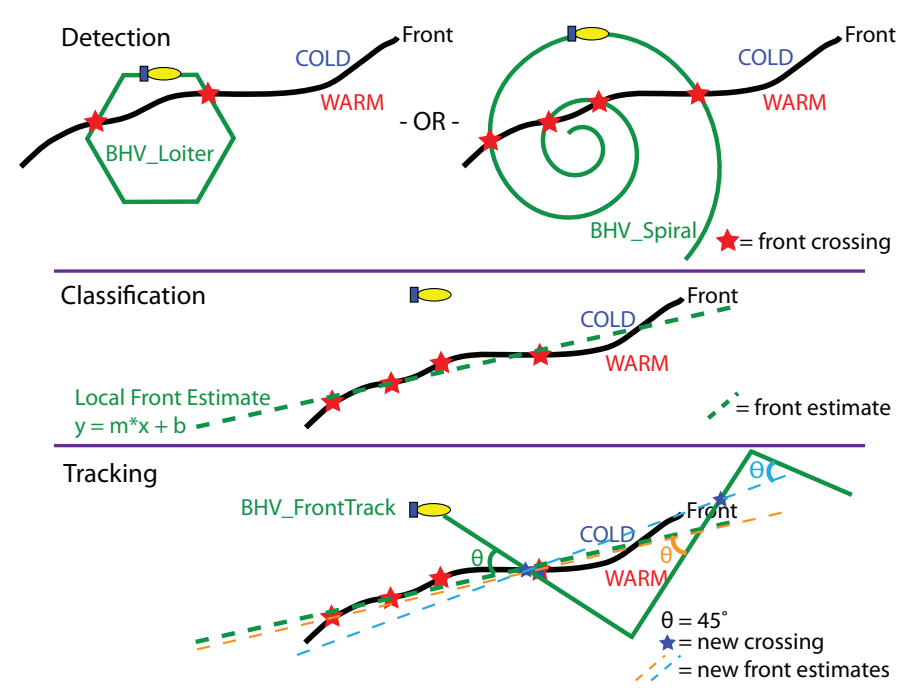

Figure 5-5: The 2D (constant depth) front tracking method, which is comprised of three phases: detection, classification, and tracking.

front crossings are detected. When completed, the AUV continues with the classification phase.

\section{Classification}

The AUV classifies the front locally as a line, based on the front crossing locations. The classification developed here is a weighted linear least squares approximation of the local front line, requiring at least three crossing points to be within a specific spatiotemporal range of the current time and AUV position to produce the linear approximation. The spatiotemporal ranges selected are based on characteristic spatiotemporal scales of the front being tracked, and thus must be set by the AUV operator prior to the start of the mission. If the number of crossing points in range drops below three, the AUV will return to a loiter or spiral pattern around the last known crossing point until three crossing are collected in range. Once the local front line has been classified, the AUV begins the tracking phase.

\section{Tracking}

The tracking phase sets the heading of the AUV to intersect the front line estimate at a $45^{\circ}$ angle. When the AUV crosses the actual front line (isotherm), it then travels a specified distance before turning to intersect the front again. The AUV adds the new crossing point to the vector of in-range crossings and updates the front line estimate to produce a new heading to intersect the front again (also at a $45^{\circ}$ angle). This results in a zigzag path along the front that follows the front as it shifts over space and time. 


\subsubsection{BHV_FrontTrackNoBdry (2D)}

To implement 2D front tracking, the autonomy behavior BHV_FrontTrackNoBdry was developed. This behavior guides a single AUV through the detection, classification, and tracking phases outlined in Section 5.5.1, resulting in a zigzag pattern tracing the front line, punctuated by loiter and/or spiral patterns when the front is lost or the behavior is reinitialized.

In the detection phase, the AUV determines the isotherm temperature that gets defined and calculated as the front line's temperature $\left(T_{\text {front }}\right)$ being the mean of the maximum $\left(T_{\max }\right)$ and minimum $\left(T_{\min }\right)$ temperatures it has encountered. That is,

$$
T_{\text {front }}=\frac{T_{\max }+T_{\min }}{2}
$$

This is a somewhat arbitrary selection of the frontal temperature, but it will always yield a value that was recently observed in the environment the AUV has explored. Alternatively, the spatial gradient of temperature across the front could be used as the tracer value for the front, but this value can only be calculated as the AUV moves perpendicularly across the front at constant depth, or if the AUV has external knowledge of the local front line heading. The AUV cannot predict if it will actually cross the front orthogonally to the front line or at a certain angle of incidence, since it has limited knowledge of the heading of the local front line and the front is dynamic in both space and time. In addition, the temperature gradient in the across-front direction is often not uniform along the front [41] [42]. Thus, it is reasonable to select an isotherm as the local front line, which will often run roughly parallel to the actual high-gradient line along the front.

Another way to detect the front position from an initial survey combines the two aforementioned methods. The AUV could perform a circle as the initial survey, calculating the temperature gradient in the azimuthal direction around the circle. The average of the temperatures at the two locations along the circle where the temperature gradient peaks can be used as the isotherm temperature that the AUV selects to track along. This azimuthal temperature gradient front detection method has not been applied in the virtual front tracking experiments described in this chapter, however it will be integrated into future iterations of BHV_FrontTrackNoBdry.

In the classification phase, a weighted linear least squares calculation is used to calculate the local front line from all front crossings 'in range' of the assigned characteristic spatiotemporal scales of the front. For example, a coastal shelfbreak front at a given depth may change significantly over a time scale of $10 \mathrm{hr}$ and a horizontal range scale of $10 \mathrm{~km}$. Using a linear decay from the AUV's current position and time (weighting 
factor of 1) to the range and time elapsed over one characteristic spatiotamporal scale or more (weighting factor of 0 ), the crossing points can be weighted such that the newest and closest crossing points have the greatest influence on the estimated local front line. This weighting allows the front line estimate to update and track the front as it changes.

As the front line estimate is updated, so is the heading of the AUV required to intersect the estimated front line at a $45^{\circ}$ angle. This results in the desired autonomous and adaptive front tracking behavior that is being sought as the AUV zigzags across the dynamic front, following its curves.

Some safeties built into this front tracking include 1) a spiral behavior that directs the AUV into an outward spiral to find the front line if it strays too far off course while tracking and 2) a re-initialization of the temperature range and detection phase when the AUV's time spent front tracking has exceeded the assigned characteristic temporal scale of temperature variation in the region.

It is also important to note that it is sometimes desirable for the AUV to track exactly along the front (e.g., when studying small-scale turbulence along the front), but often it is desirable for the AUV to cross back and forth across the front to increase coverage, since a frontal boundary is often very complex and not a well-defined or ideal smooth curve over the horizontal plane. Thus, using methods such as applying a proportional-integral-derivative (PID) controller to tune out the zigzagging motion of the AUV is not a useful solution for many front tracking cases, whereas a zigzagging motion of fixed or dynamically variable amplitude coupled with a front line estimate, as described here, may be adjusted for the desired across-front coverage.

Though BHV_FrontTrackNoBdry runs independently on each AUV tasked with autonomous and adaptive front tracking, it can be run with other behaviors such as BHV_FollowTheLeader (Section 5.5.3) or BHV_RubberBand (see [95]) to coordinate the global motion of multiple AUVs. Using multiple AUVs can provide synoptic sampling coverage over a larger spatial scale than a single AUV when the AUVs are distributed within the front's characteristic range of each other, as sketched in Fig. 5-2.

\subsubsection{BHV_FollowTheLeader (Multi-AUV)}

BHV_FollowTheLeader was designed for use with two or more AUVs, though it has only been tested with two. One AUV is assigned as the leader, and the other(s) is(are) designated a follower. As shown in Fig. 5-6, the follower-to-leader absolute bearing (angle clockwise from north), $b_{F 2 L}$, is calculated, and a heading bias (clockwise from north), HBiasfollower, equal to the bearing angle is superimposed on the follower's final heading objective function. Similarly, the leader-to-follower absolute bearing, $b_{L 2 F}$, is calculated, and a 
heading bias, HBiasleader, equal to the bearing angle plus $180^{\circ}$ is superimposed on the leader's final heading objective function. The heading biases are then given a range of HBias $\pm 90^{\circ}$ where the objective function weighting is constant before tapering to a weight of 0 at $H B i a s \pm 180^{\circ}$. That is,

$$
\begin{aligned}
& b_{L 2 F}=b_{F 2 L}+180^{\circ}, \\
& \text { HBiasfollower }_{\text {B }}=b_{F 2 L},
\end{aligned}
$$

and

$$
\text { HBiasleader }_{\text {B }}=b_{L 2 F}+180^{\circ}=\text { HBias }_{\text {follower }} .
$$

The resulting objective function over heading for BHV_FollowTheLeader is shown in Fig. 5-7. Even though HBiasleader $_{\text {HBiasfollower }}$ at any given moment in time, each AUV is only responsible for calculating its own 'leader' or 'follower' HBias value, according to how it has been assigned. This is because only AUV position values—not the HBias values—are shared over the acoustic link.

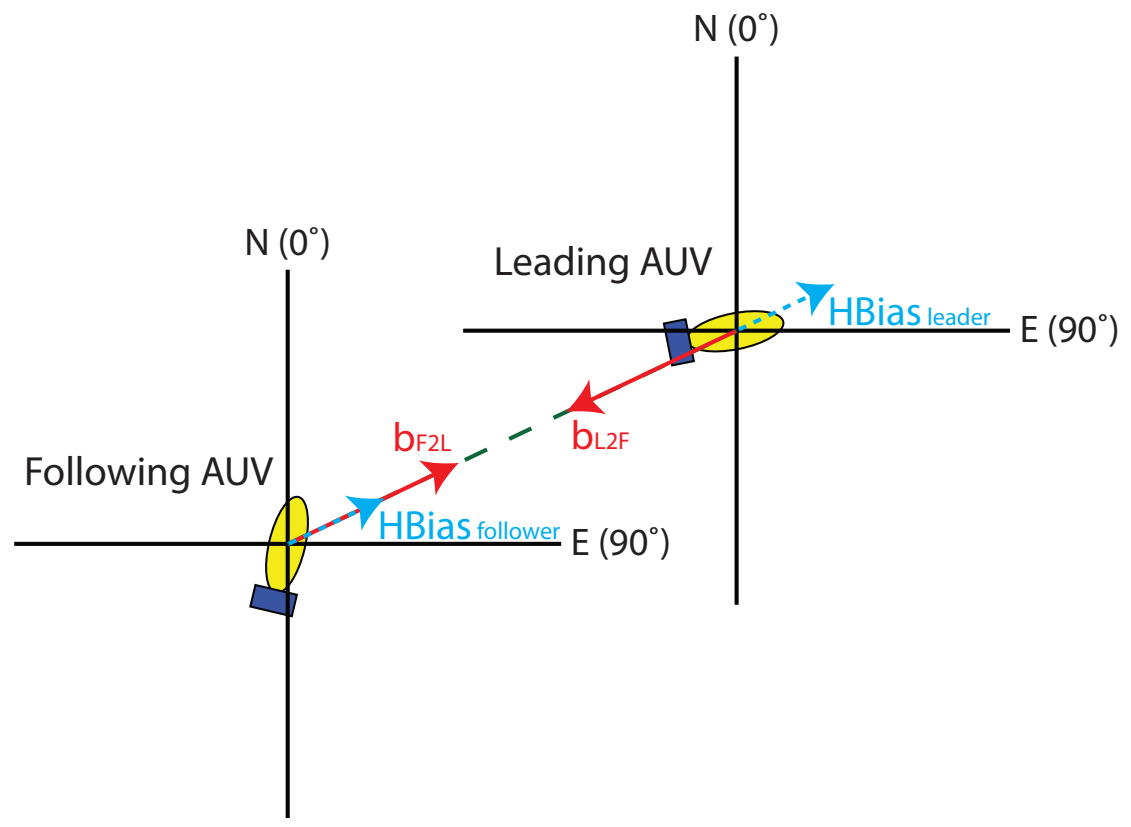

Figure 5-6: A sketch depicting desired heading angles (clockwise from north, light blue dotted arrows), HBias, as the desired directions of motion for the leader and follower AUVs, and the AUVs' absolute bearings (angles clockwise from north, red solid arrows) to each other, $b_{L 2 F}$ and $b_{F 2 L}$, as calculated by BHV_FollowTheLeader.

This creates a behavior in which the leader AUV tends to move away from the follower while the follower moves toward the leader. If more followers were chained onto the first follower, the idea is that the AUVs 


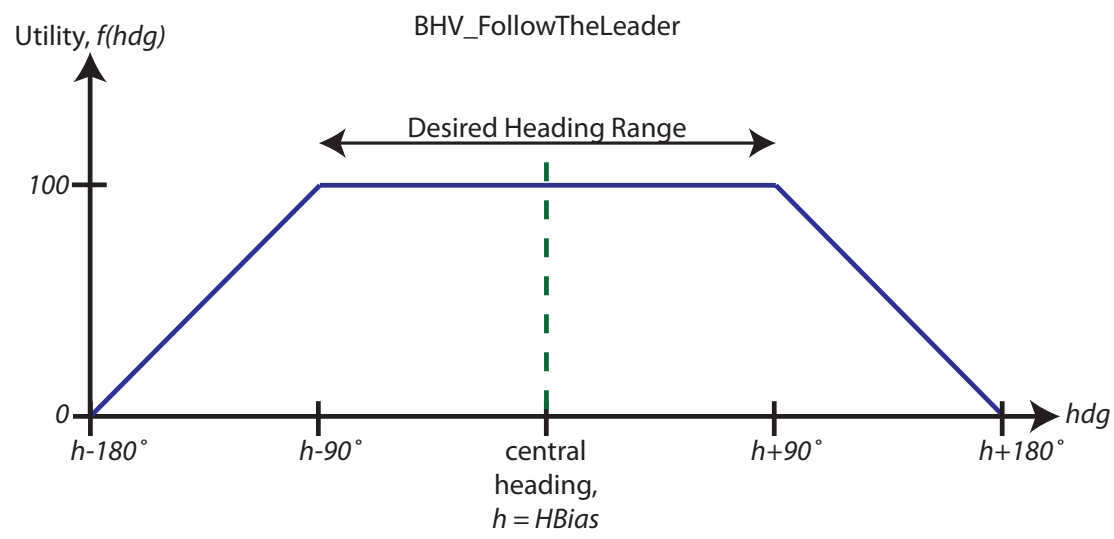

Figure 5-7: Objective function over heading for BHV_FollowTheLeader, where heading, $h$, —on which the objective function and heading bias are centered-is set by each AUV. At any given point in time, $h=$ HBiasleader $_{\text {B }}$ Hiasfollower. Thus, due to the utility of the function being flat on the interval $h \pm 90^{\circ}$ and tapering linearly to zero at $h \pm 180^{\circ}$, the leader will travel generally away from the follower while the follower roughly moves in the direction of the leader.

would all be able to move along a front or other oceanographic boundary in follow-the-leader style, while still zigzagging across the boundary, adapting to the front's local position. Ideally, a separation distance constraint like that of BHV_RubberBand (see [95]) would be added to the AUVs' behavior set to maintain synoptic sampling coverage in the along-front direction as well.

\subsection{D Front Tracking}

Including the third (vertical) dimension in characterizing a front is important due to features that occur in the vertical water profile, such as thermoclines, pycnoclines, Chlorophyll maxima, etc. Using a single AUV executing an adaptive horizontal helix behavior (BHV_FrontTrackHelix) along the front line and with the central axis at a fixed depth, the front through that depth can be estimated as a plane.

Multiple AUVs may also be employed, each at a different depth, but roughly vertically aligned, to create a 3D map of a front. Each AUV would independently perform either 2D adaptive zigzag front tracking or 3D adaptive helix front tracking, while a separate behavior would be designed to keep them roughly stacked vertically. This, however, is beyond the scope of this thesis and will not be addressed further here.

\subsubsection{BHV_FrontTrackHelix (3D)}

The 3D, single-AUV front tracking behavior, BHV_FrontTrackHelix, designed in this work is based on the same weighted linear least squares estimation of the front line at a given depth that is used in the adaptive front 
tracking zigzag behavior, but the AUV's position is guided by a horizontal helix around the chosen depth's front line. In designing this behavior for an AUV, the helix characteristics are constrained by:

- the AUV's speed,

- the AUV's maximum ascent angle $\left(\sim 30^{\circ}\right)$,

- the desired helix radius (manually selected based on water depth and depth of AUV),

- and the front line estimate at the helix's center depth.

The AUV's desired position on the helix, $\left(x, y, z, t_{\text {now }}\right)$, is calculated as follows. The helix must be rotated in the horizontal plane to align with the front line estimate, $y_{c t r}$, using the rotation matrix, $R$, and rotation angle, $\theta$. The rate of travel around the helix must also be slowed to account for the AUV's speed and ascent angle limitations, which are taken into account when calculating the period, $p$, and, subsequently, the angular rate of travel, $\omega$, on the helix. Table 5.1 serves as a reference for the variables, which are related in the calculation of the desired AUV position, Equations 5.5-5.15.

First, approximate the angular oscillation rate, $\omega$, of the AUV moving along the helix path at speed $v$ :

$$
\begin{gathered}
p \approx \frac{2(2 r)}{v \sin \left(\alpha \frac{\pi}{180^{\circ}}\right)} \\
\omega=\frac{2 \pi}{p} \approx \frac{2 \pi}{p} P, \text { where } P=2 \\
\therefore \omega \approx \frac{\pi v P \sin \left(\alpha \frac{\pi}{180^{\circ}}\right)}{2 r}=\frac{\pi v \sin \left(\alpha \frac{\pi}{180^{\circ}}\right)}{r}
\end{gathered}
$$

Next, calculate AUV position on the helix path in the horizontal plane based on the time duration since the helix began, with the helix's center axis aligned with the $\mathrm{x}$-axis $(x, y)$ :

$$
\begin{gathered}
\Delta t=t_{\text {now }}-t_{0} \\
{\left[\begin{array}{l}
x_{h} \\
y_{h}
\end{array}\right]=\left[\begin{array}{l}
c \omega \Delta t \\
r \sin (\omega \Delta t)
\end{array}\right]}
\end{gathered}
$$

Then, rotate and translate the AUV position on the helix path in the horizontal plane through angle $\theta$ to align the helix's center axis with $y_{c t r},\left(x_{r o t}, y_{r o t}\right)$ : 
Table 5.1: Helix position calculation variables

\begin{tabular}{|c|c|}
\hline Variable & Definition \\
\hline$y_{c t r}$ & Front line estimate at a given depth $\left(-z_{c t r}\right),[\mathrm{m}]$ \\
\hline$-z_{c t r}$ & Helix center depth, $[\mathrm{m}]$ \\
\hline$m$ & Slope of front line estimate in horizontal space, $[\mathrm{m} / \mathrm{m}]$ \\
\hline$b$ & $\begin{array}{l}\text { Intercept of front line estimate with y-axis in horizontal space, } \\
{[\mathrm{m}]}\end{array}$ \\
\hline$t_{0}$ & Helix start time, [Unix sec] \\
\hline$t_{\text {now }}$ & Current time, [Unix sec] \\
\hline$\Delta t$ & Time since helix start, [sec] \\
\hline $2 \pi c$ & Constant, separation distance of helix loops (user-selected), [m] \\
\hline$r$ & Radius of helix, $[\mathrm{m}]$ \\
\hline$\alpha$ & Ascent angle of AUV, $\leq 30^{\circ},\left[^{\circ}\right]$ \\
\hline$R$ & Rotation matrix \\
\hline$\theta$ & $\begin{array}{l}\text { Rotation angle to align the horizontal helix axis with the front } \\
\text { estimate line, }[\mathrm{rad}]\end{array}$ \\
\hline$p$ & $\begin{array}{l}\text { Period of helix oscillation based on AUV speed, calculated } \\
\text { approximating the sinusoidal motion of the helix as a zigzag, } \\
\text { [sec] }\end{array}$ \\
\hline$\omega$ & $\begin{array}{l}\text { Angular rate of oscillation based on AUV speed, calculated } \\
\text { approximating the sinusoidal motion of the helix as a zigzag, } \\
{[\mathrm{rad} / \mathrm{sec}]}\end{array}$ \\
\hline$P$ & $\begin{array}{l}\text { Petillo scaling factor, to account for variations in AUV speed } \\
\text { along the helix path and reduce doubling-back motion }(P=2 \\
\text { for the Bluefin } 21 \text { " AUVs in our virtual experiments })\end{array}$ \\
\hline$v$ & AUV's (desired) speed, $[\mathrm{m} / \mathrm{s}]$ \\
\hline$\left(x, y, z, t_{\text {now }}\right)$ & $\begin{array}{l}\text { Desired AUV position on the helix at the current time, }([\mathrm{m}] \text {, } \\
[\mathrm{m}],[\mathrm{m}],[\mathrm{sec}])\end{array}$ \\
\hline
\end{tabular}

$$
\begin{gathered}
R=\left[\begin{array}{cc}
\cos (\theta) & -\sin (\theta) \\
\sin (\theta) & \cos (\theta)
\end{array}\right] \\
y_{c t r}=m x+b \\
\theta=\tan ^{-1}(m), \text { where }-\pi \leq \theta \leq \pi
\end{gathered}
$$




$$
\begin{aligned}
{\left[\begin{array}{l}
x_{r o t} \\
y_{r o t}
\end{array}\right] } & =R \times\left[\begin{array}{l}
x_{h} \\
y_{h}
\end{array}\right]+\left[\begin{array}{l}
0 \\
b
\end{array}\right] \\
& =\left[\begin{array}{l}
x_{h} \cos (\theta)-y_{h} \sin (\theta) \\
x_{h} \sin (\theta)+y_{h} \cos (\theta)+b
\end{array}\right] \\
& =\left[\begin{array}{l}
c \omega \Delta t \cos (\theta)-r \sin (\omega \Delta t) \sin (\theta) \\
c \omega \Delta t \sin (\theta)+r \sin (\omega \Delta t) \cos (\theta)+b
\end{array}\right]
\end{aligned}
$$

and calculate the vertical position of the AUV on the helix, $z$ :

$$
z=r \cos (\omega \Delta t)-z_{c t r}
$$

Finally, assign the proper values to the desired current AUV position along the helix, which drive the AUV's motion using autonomy:

$$
\left(x, y, z, t_{\text {now }}\right)=\left(x_{\text {rot }}, y_{\text {rot }}, z, t_{\text {now }}\right)
$$

Section 5.7 describes the resulting virtual experiments and data collected from testing the $2 \mathrm{D}, 3 \mathrm{D}$, and multi-AUV front tracking behaviors described above.

\subsection{Virtual Experiments \& Results}

In order to compare preplanned front mapping missions to adaptive front tracking missions, a number of virtual experiments were run with one AUV performing a fixed preplanned horizontal zigzag while a second AUV used adaptive front tracking behaviors to track the front it detected. The AUV conducting the fixed preplanned zigzag shared its start location with that of the adaptive AUV. The heading, amplitude, and period of the preplanned zigzag were selected based on operator estimation of the front position from a random snapshot of the front at the AUVs' operational depth. All AUVs were assigned the same operational depth (or helix center-axis depth) in a given virtual experiment, and the virtual experiments ended when the fixed zigzag mission finished or the virtual experiment exceed a specified amount of time (for other comparative missions not involving the fixed zigzag mission). The performance of fixed zigzag to adaptive front tracking missions was evaluated and compared using a number of performance metrics, which are described in Section 5.8.1. 4D MSEAS ocean models integrated into the MIT Laboratory for Autonomous Marine Sensing Systems (LAMSS) AUV virtual experiment environment, described in Section 5.7.1 and Appendix A, were used as realistic oceanographic environments for testing all of the behaviors described in Sections 5.5 and 5.6. 


\subsubsection{MSEAS 4D Ocean Model Environment}

The MSEAS group at MIT uses oceanographic data coupled with fluid dynamics and physical oceanography principles to create 4D, spatiotemporally dynamic, gridded ocean models [60]. These models are provided in NetCDF format with a number of tools written in MATLAB to read, interpolate, and plot the model data. MSEAS models were incorporated into the LAMSS AUV virtual experiment setup to provide a realistic testing environment for the environmentally adaptive feature tracking missions, including data extraction and visualization tools. The details of this LAMSS-MSEAS interface and the associated tools are in Appendix A.

MSEAS Modeling System The Multidisciplinary Simulation, Estimation, and Assimilation System (MSEAS) [96] is used to study and quantify tidal-to-mesoscale processes over regional domains with complex geometries and varied interactions. Its modeling capabilities include implicit two-way nesting for multiscale hydrostatic primitive equation (PE) dynamics with a nonlinear free-surface [97] and a high-order finite element code on unstructured grids for non-hydrostatic processes also with a nonlinear free-surface [98-100]. Other MSEAS subsystems include: initialization schemes [101], nested data-assimilative tidal prediction and inversion [102]; fast-marching coastal objective analysis [103]; stochastic subgrid-scale models (e.g., [104, 105]); generalized adaptable biogeochemical modeling systems; Lagrangian Coherent Structures; non-Gaussian data assimilation and adaptive sampling [106-108]; dynamically-orthogonal equations for uncertainty predictions [109-111]; and machine learning of model formulations [112]. The MSEAS software is used for basic and fundamental research and for realistic simulations and predictions in varied regions of the world's ocean [113-120], including monitoring [121], naval exercises including real-time acoustic-ocean predictions [122] and environmental management [123].

Model Selection for Virtual Experiments For the application of front tracking, the Mid-Atlantic Bight (MAB) region was selected for the shelfbreak front off the Atlantic coast of the U.S. and the robust model available for this region. The data input to the MAB model are from the Shallow Water 2006/Autonomous Wide Aperture Cluster for Surveillance (SW06/AWACS) exercise that took place in the MAB in August and September 2006, and the time frame selected from the models covers approximately August 28-September 8, 2006. Within this window of time, Tropical Storm Ernesto passed through the MAB region from roughly September 1-3, 2006, adding variation and mixing along the Gulf Stream and shelfbreak to the data collected for the resulting models. The SW06 data has been gridded by the MSEAS group for the full region provided in the MAB model. An updated version of this model is available on the MSEAS SW06 Re-Analyses website [124], along with the associated MATLAB tools for plotting and interpolation. 
The environmental parameters available for the MSEAS MAB model are temperature, $T$, salinity, $S$, and zonal, meridional, and vertical currents, $u, v$, and $w$, respectively, at each grid point, (Longitude, Latitude, Depth, time). The shelfbreak front is most apparent when looking at salinity (see Fig. 5-8), but it is also relatively clear in the temperature signature [43]. Since many temperature sensors are more robust than salinity sensors (which are often sensitive to temperature changes), temperature is used as the parameter that guides the decisions in the front tracking behaviors. However, accurate salinity, density, or sound speed measurements can also be used as input to the same front tracking algorithms when they are frontal indicators.

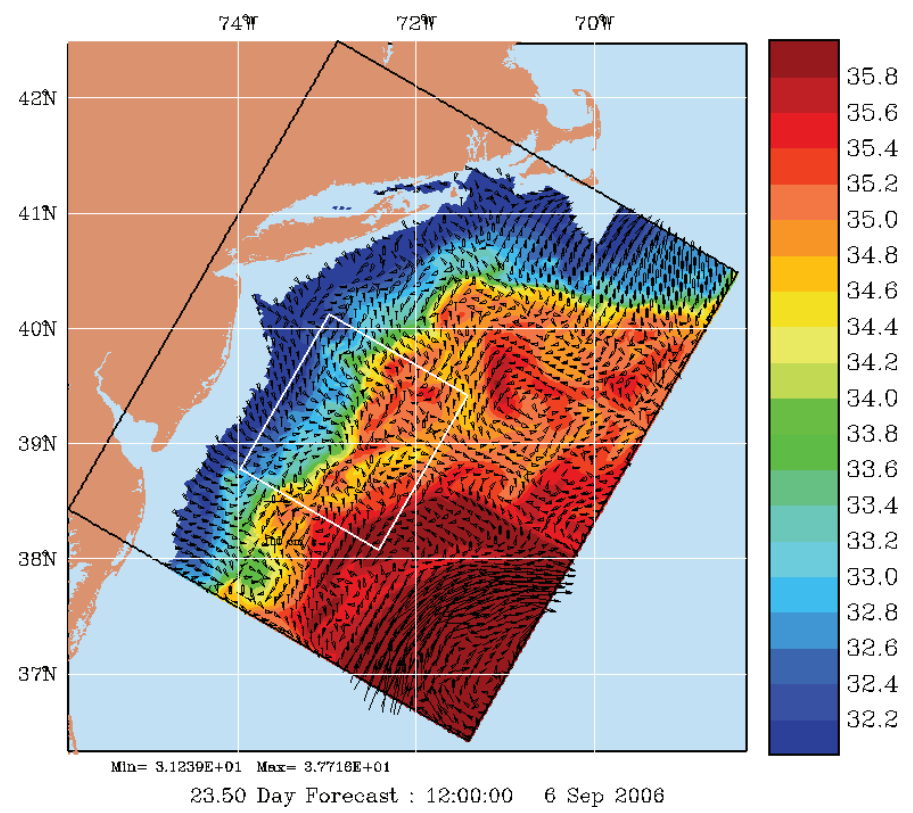

Figure 5-8: A horizontal slice of the MSEAS SW06 model data for the Mid-Atlantic Bight region. The color variations indicate the salinity values. The SW06 domain is the full domain bounded by the blackbordered box, and the AW06 domain is bounded by the smaller white-bordered box. Image credit: MSEAS group [124].

SW06/AWACS Simulation The real-time Shallow Water 2006 (SW06)/AWACS exercise was carried out in the New Jersey Shelf/Hudson Canyon region over the time period August-September 2006 [125-128]. Based on this experiment, realistic ocean fields were created using the free-surface MSEAS PE model employing two-way implicit nesting with tidal and atmospheric forcing. The coarse domain (SW06) is a $522 \mathrm{~km} \times$ $447 \mathrm{~km}$ domain with $3 \mathrm{~km}$ resolution. The fine domain (AW06) is a $172 \mathrm{~km} \times 155 \mathrm{~km}$ domain with $1 \mathrm{~km}$ resolution. Both domains employ 100 vertical levels in a double- $\sigma$ configuration (see app. 1.1 in [97]). The bathymetry used for this simulation was a combination of the NOAA [129] Coastal Relief Model combined with V8.2 (2000) of the Smith and Sandwell [130] topography in the deep regions. The estimation 
of the initial conditions was based on two objective analyses, one inshore and one offshore of the expected shelfbreak. The initial conditions were estimated using in situ data from Rutgers SeaGliders, National Marine Fishery Services (NMFS) cruises, CTD casts collected aboard the research vessels Knorr, Quest and Tioga, as well as Scanfish data. These observations were augmented with additional synoptic data from the World Ocean Database (WODB) [131], the Global Temperature and Salinity Profile Programme (GTSPP) [132] and pseudo profiles to bolster the shelfbreak front. The synoptic data were melded with the World Ocean Atlas (WOA) [133,134] climatology modified to match the 2006 slope conditions. Feature models [135-137] for the shelfbreak front, Gulf Stream and slope recirculation gyre were employed to ensure realistic synoptic structures. The position of the Gulf Stream was estimated based on SST and NAVOCEANO feature analyses. The simulations were forced with atmospheric fluxes derived from the Weather Research and Forecasting system (WRF) [138] and the Navy Operational Global Atmospheric Prediction System (NOGAPS) [139], and laterally forced with OTIS tides [140]. The synoptic data is assimilated at $3 \mathrm{hr}$ intervals to control uncertainties. Extensive sensitivity studies were performed to select the model parameters which best match the observations. New time-dependent sponging and lateral radiative boundary conditions were formulated to prevent spurious reflections at the boundaries.

AUV Operation Region Within the MAB model SW06 domain, two AUV operation boxes were defined in the area where the Pioneer Array is planned to be deployed with AUVs and gliders at the shelfbreak south of Cape Cod, MA. In this region, the model has $3 \mathrm{~km}$ grid resolution. A third AUV operation box was defined southwest of the Pioneer Array along the shelfbreak east of New Jersey (the AW06 domain) where a $1 \mathrm{~km}$ grid resolution model is available in addition to the $3 \mathrm{~km}$ resolution SW06 model. There is a distinct thermal and salinity front present along the entire shelfbreak, highlighted in Fig. 5-9, that was used for testing the front tracking behaviors described in Sections 5.5 and 5.6.

\subsubsection{Preplanned Missions}

The preplanned missions used in the virtual experiments consisted of either a zigzag across a straight line (Fig. 5-10) or a zigzag following an isothermal contour (Fig. 5-11). The straight-line zigzag was at constant depth and heading with a fixed amplitude, distance, and number of straight leg segments. The isotherm-following zigzag was also at constant depth, although the zigzag amplitude varied (but was larger for the most part) and the number of straight leg segments was greater. The isothermal contour position was assumed to be known and static for planning purposes (taken from a random snapshot of a horizontal model slice at the 


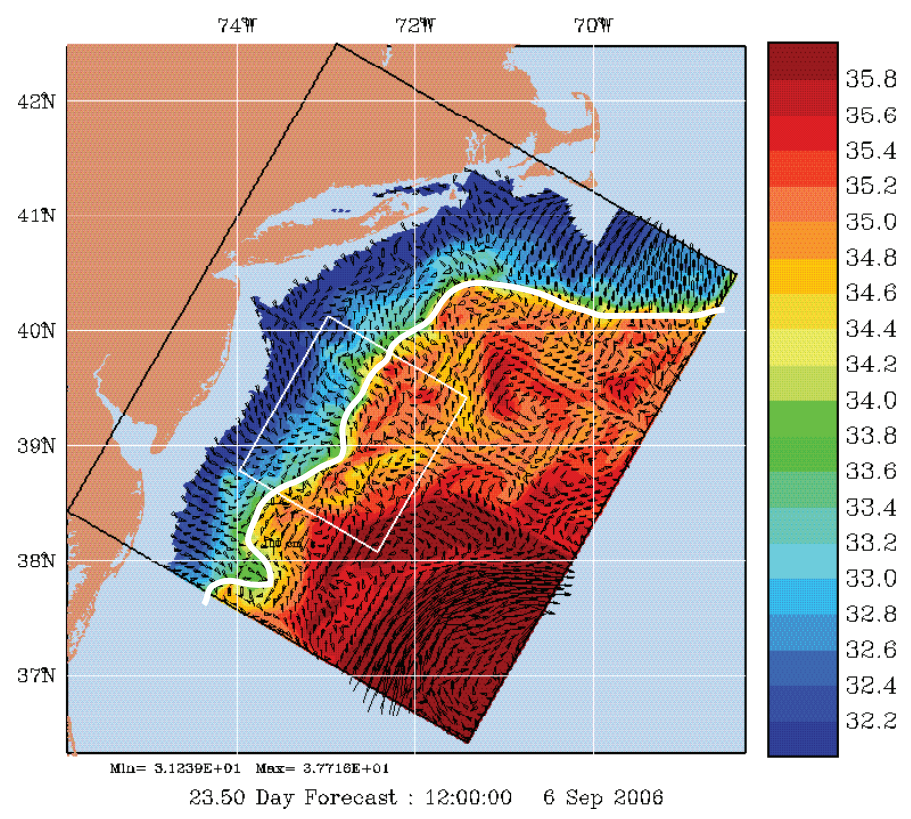

Figure 5-9: The shelfbreak thermal and salinity front, highlighted in white, through the Mid-Atlantic Bight region. The color variations indicate the salinity values. The SW06 domain is the full domain bounded by the black-bordered box, and the AW06 domain is bounded by the smaller white-bordered box. Adapted from [124].

AUVs' operational depth), but a large coverage area was selected for the survey to maximize sampling distance across the front and minimize loss of the front line. The isotherm-following zigzag was scripted from handselected waypoints on the same snapshot to follow the frontal contour (with front intersect angles attempting to stay near $45^{\circ}$ ), so the resulting survey area covered by either preplanned mission does not always reflect the position of the dynamic front over time.

\subsubsection{New Adaptive Missions}

The adaptive front tracking missions consist of one or more AUVs tasked with detecting and tracking an isothermal (or isohaline or isopycnal) contour representing the front line, using temperature (or salinity or density) measurements it collects in situ. Whether using one or many AUVs for these missions, each AUV runs either the 2D (BHV_FrontTrackNoBdry) or 3D (BHV_FrontTrackHelix) front tracking behavior to keep it tracking the front locally while (in the multi-AUV case) another behavior such as BHV_FollowTheLeader coordinates the AUVs' motion relative to each other for increased spatiotemporal coverage in the dynamic virtual experiment (and real) environment (see Table 5.2). 


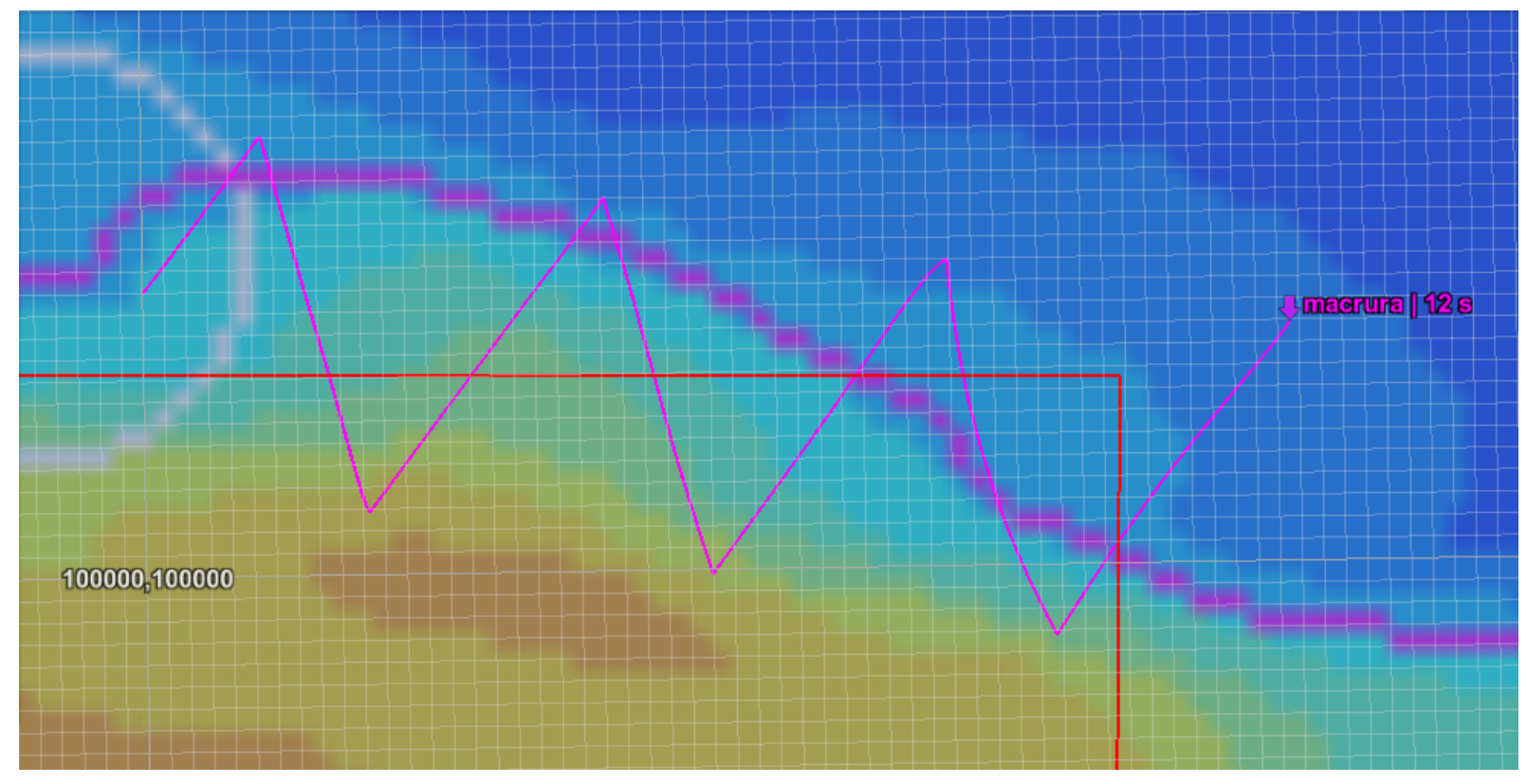

Figure 5-10: A preplanned AUV zigzag mission (magenta 'Macrura' line) at constant depth, heading, amplitude, length, width, and period. The zigzag mission is configured based on a recent snapshot of the temperature field at the selected AUV operation depth. The small grid squares are $1 \mathrm{~km} \times 1 \mathrm{~km}$. The blurry purple line follows a frontal isotherm.

Table 5.2: Adaptive Front Tracking Behavior Combinations

\begin{tabular}{|l|l|l|}
\hline Dimensions & Single/Multiple AUVs & Behaviors \\
\hline \hline 2D & Single & BHV_FrontTrackNoBdry \\
\hline 2D & Multiple & $\begin{array}{l}\text { BHV_FrontTrackNoBdry with } \\
\text { BHV_FollowTheLeader or BHV_RubberBand }\end{array}$ \\
\hline 3D & Single & BHV_FrontTrackHelix \\
\hline 3D & Multiple & $\begin{array}{l}\text { BHV_FrontTrackHelix with behavior for } \\
\text { vertical AUV distribution }\end{array}$ \\
\hline 3D & Multiple & $\begin{array}{l}\text { BHV_FrontTrackNoBdry with behavior for } \\
\text { vertical AUV distribution }\end{array}$ \\
\hline
\end{tabular}




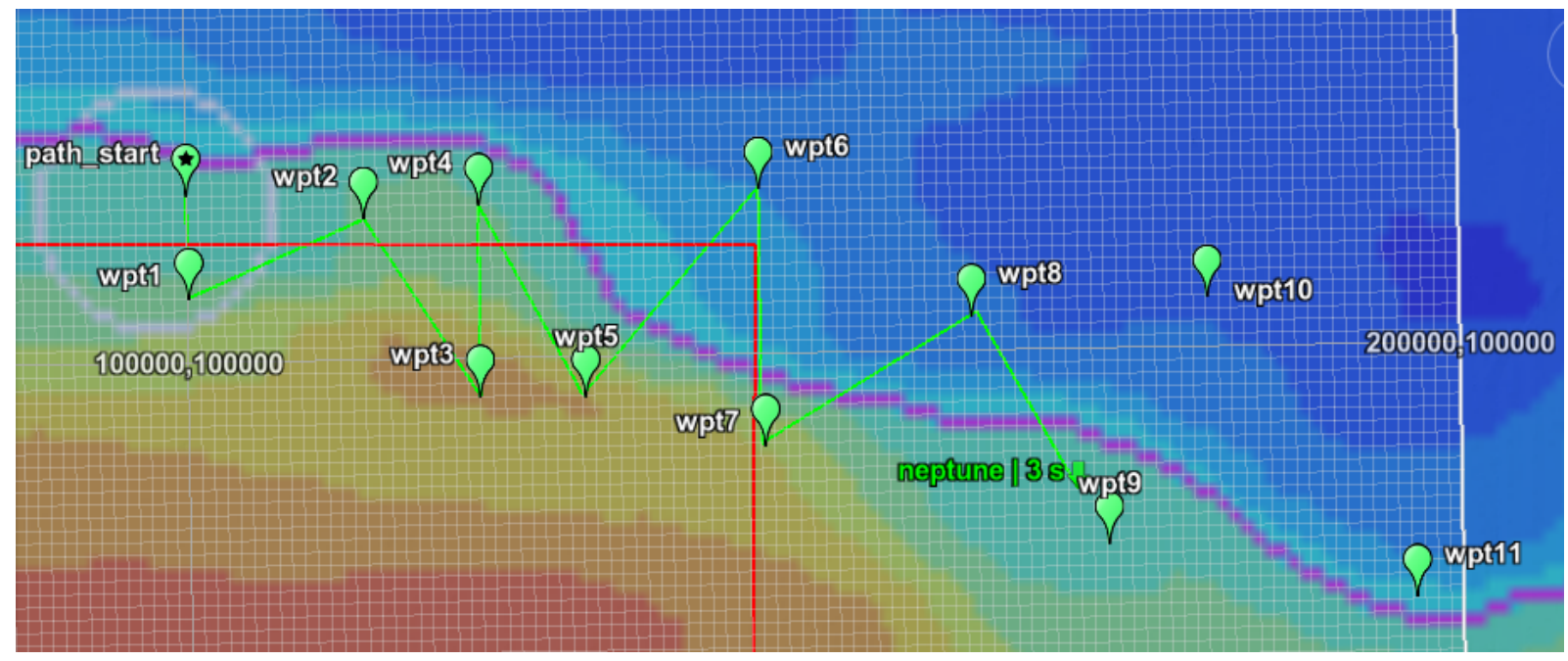

Figure 5-11: A preplanned AUV zigzag mission along an isothermal contour (green 'Neptune' line connecting waypoints) at constant depth and amplitude. The zigzag mission (originally) along a frontal isothermal (blurry purple line) is configured based on a recent snapshot of the temperature field at the selected AUV operation depth. In this snapshot, it is apparent that the front has advected away from the location it was in when this mission was originally planned. The small grid squares are $1 \mathrm{~km} \times 1 \mathrm{~km}$. 


\section{Single AUV}

When only one AUV is used for 2D front tracking, the AUV attempts to track along the front in horizontal space, crossing the front locally at an $\sim 45^{\circ}$ angle to the front line. Since there is a fixed distance traveled before the AUV turns around to re-cross the front, the resulting motion under ideal conditions creates an approximately constant amplitude zigzag pattern that travels along the front boundary as the front shifts its location in time and space (see Fig. 5-12). Good front tracking conditions generally consist of low currents in horizontal space, such that the front doesn't move faster than the AUV can follow, and a gradually curving front line lacking isolated pockets of high or low temperatures.

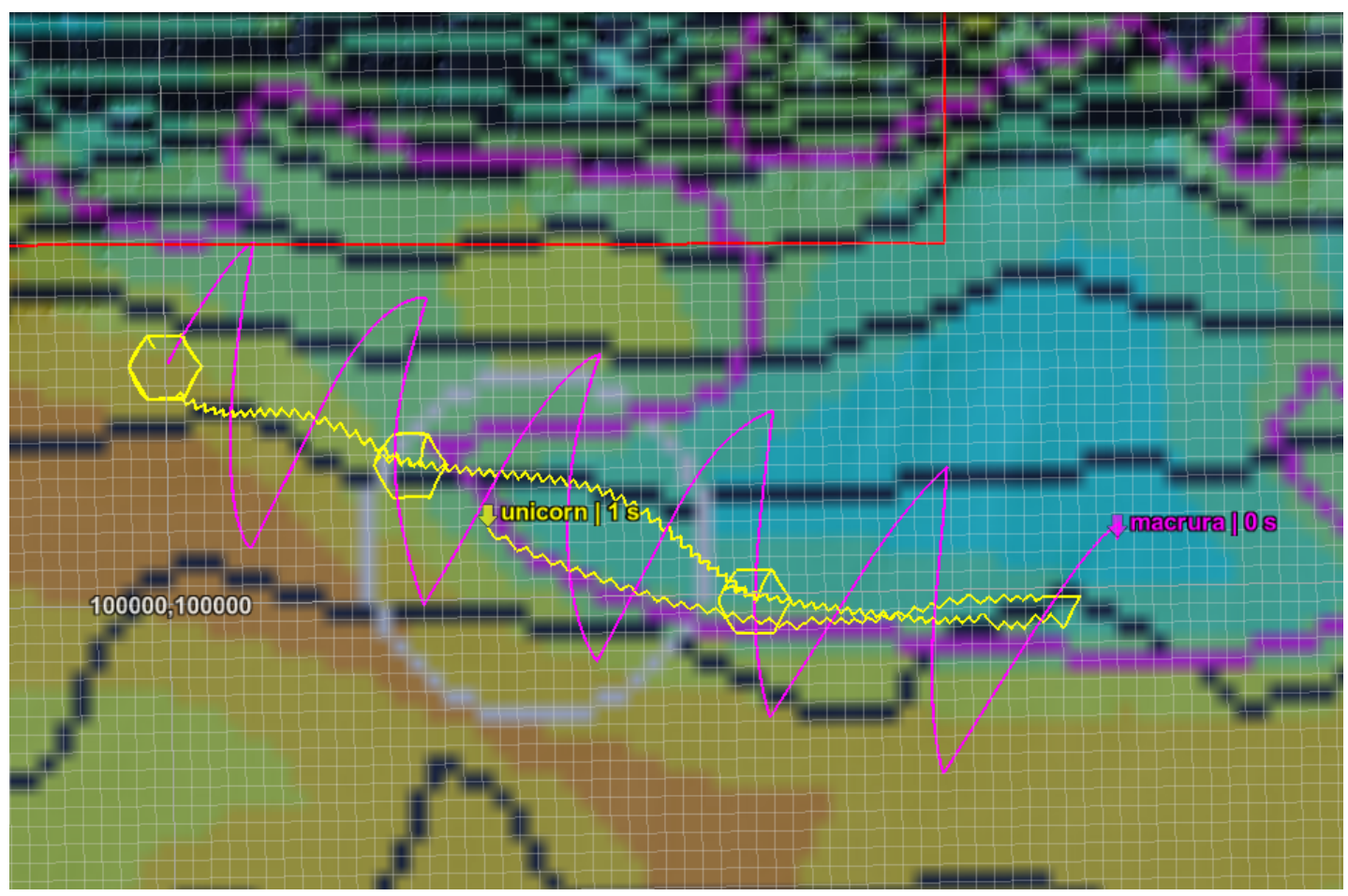

Figure 5-12: An adaptive 2D front tracking mission with nearly ideal front tracking at constant depth. The adaptive AUV path is the yellow 'Unicorn' line and the preplanned AUV path is the magenta 'Macrura' line, while the frontal isotherm location is highlighted (at the time of this snapshot) by the blurry purple line.

When conditions are poor for front tracking, the AUV's adaptive front tracking motion tends to yield more clusters of overlapping loiter patterns, as seen in Fig. 5-13. This occurs most frequently when the front line curves sharply or creates a closed loop on the order of $10 \mathrm{~km}$ or less, or when horizontal currents are strong enough to move the front line away from the AUV faster than the AUV can move.

In the case of 3D front tracking, a single AUV follows a helical path with the helix axis locally centered about a fixed-depth frontal isotherm. A close-up GEOV screenshot of this helix motion is shown in Fig. 5-14. 


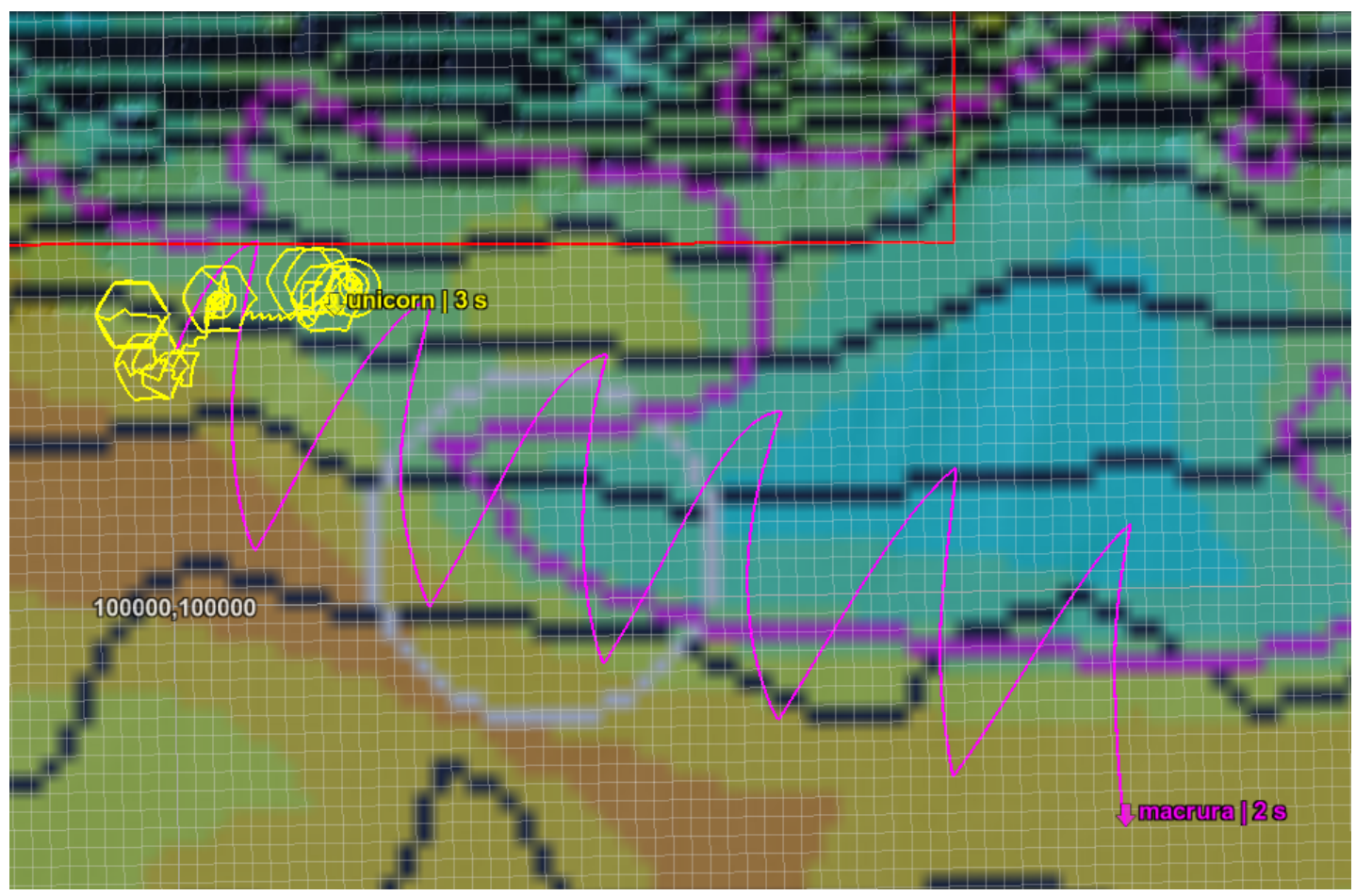

Figure 5-13: An adaptive 2D front tracking mission with poor front tracking at constant depth. The adaptive AUV path is the yellow 'Unicorn' line and the preplanned AUV path is the magenta 'Macrura' line, while the frontal isotherm location is highlighted (at the time of this snapshot) by the blurry purple line.

Under good tracking conditions, the resulting AUV path will largely look like a meandering slinky. However, under bad 3D front tracking conditions, the AUV spends most of its time loitering at constant depth to try to determine where the local front line is, similar to poor $2 \mathrm{D}$ front tracking runs.

\section{Multiple AUVs}

Multi-AUV front tracking has only been successfully demonstrated thus far with the 2D adaptive front tracking algorithms. As mentioned previously, the concept for this case is to have multiple AUVs moving along the front line at a fixed depth to create a 'global' front tracking behavior that has all the AUVs lined up traveling the same direction along the front (follow-the-leader configuration). The superimposed 'local' behavior for each AUV is simply the $2 \mathrm{D}$ adaptive front tracking zigzag motion across the local front line. This concept is illustrated in Fig. 5-2, and AUV paths from an associated virtual experiment run with 2 AUVs are shown in Fig. 5-15. Similarly, multiple AUVs could be run in follow-the-leader configuration while individually running the $3 \mathrm{D}$ helix front tracking behavior to add a range of depth samples to the collected data, but this has not been tested in virtual experiments. 


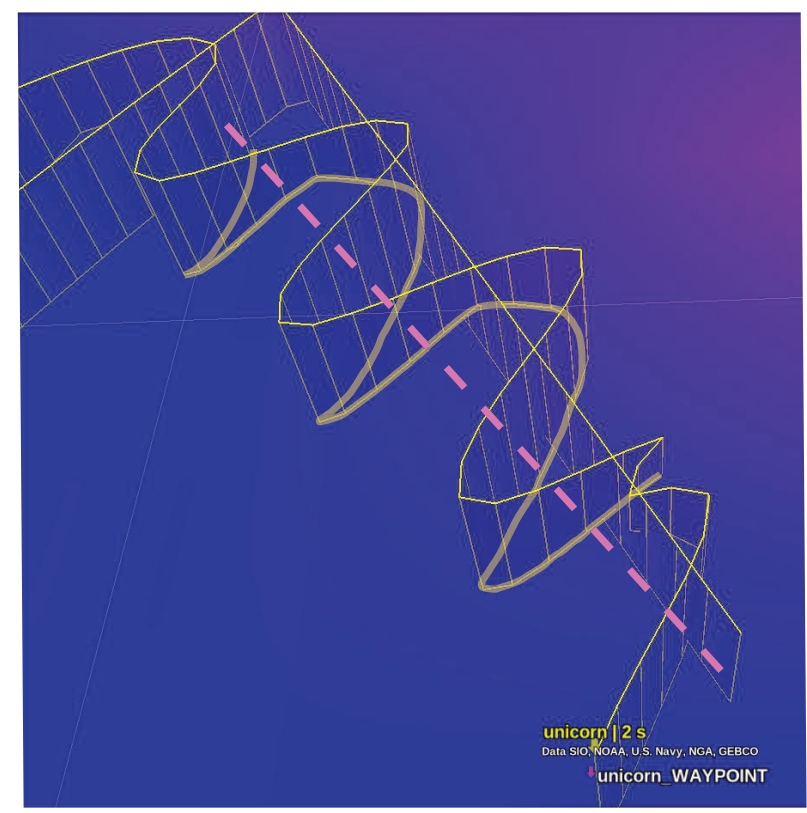

Figure 5-14: A closeup of a 3D helical front tracking path (thick yellow 'Unicorn' line) about a constant depth. The central axis of the helix (dashed line) is aligned with the front estimate line locally.

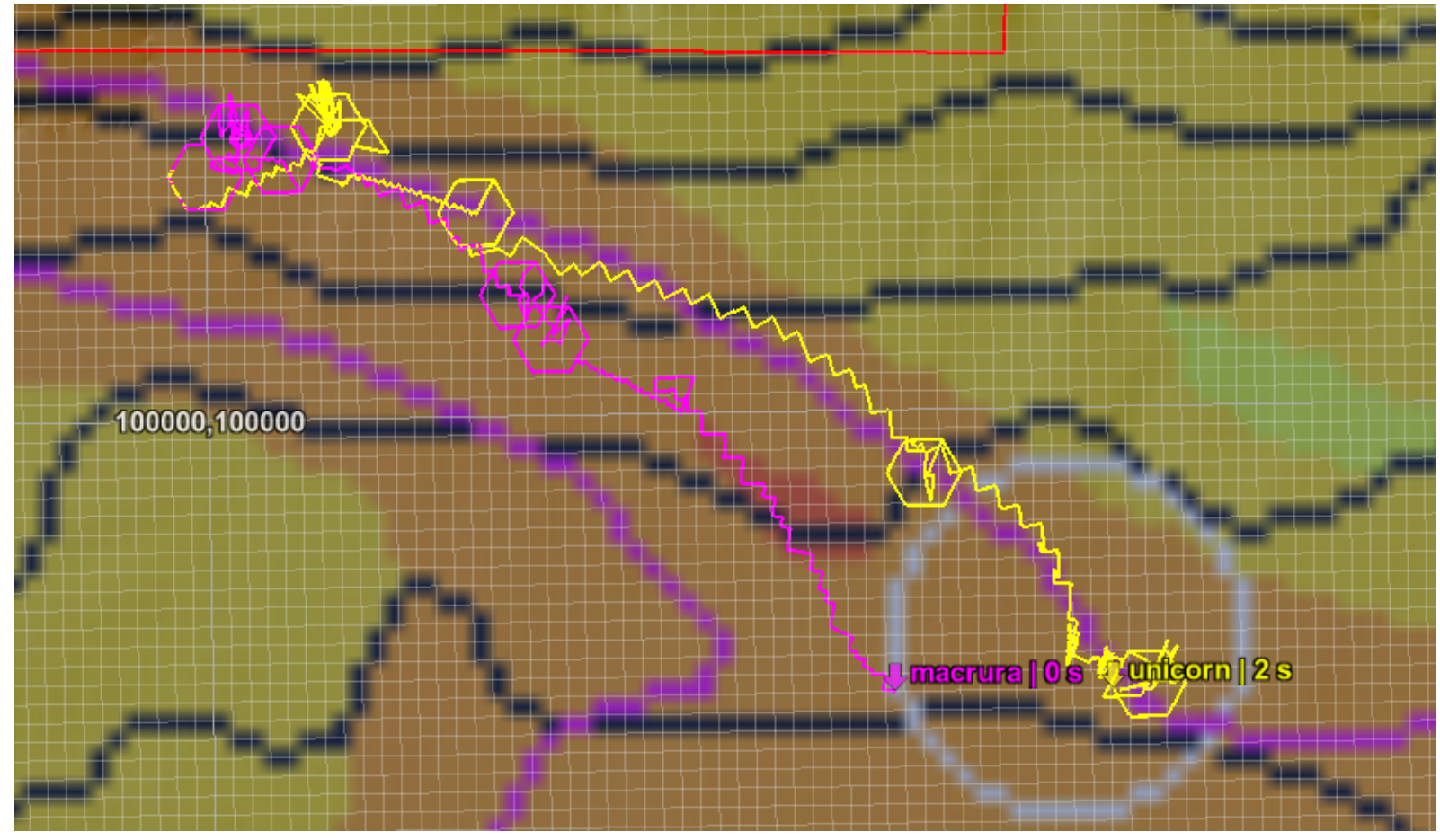

Figure 5-15: A snapshot of the paths of two AUVs performing adaptive 2D front tracking and exhibiting follow-the-leader coordination as they move in the same direction along the front. The two AUV paths are the yellow 'Unicorn' line and the magenta 'Macrura' line, and the projected frontal isotherm location is highlighted (at the time of this snapshot) by the blurry purple line. In this case, Unicorn was the leader and Macrura was the follower. The motion of the front over time is evident from the spatial offset of the paths traced by the two AUVs, where Macrura was trailing Unicorn along the front. 


\subsubsection{Mission Configurations}

This section details the specific virtual experiment mission configurations used to test the adaptive missions in various configurations and evaluate the adaptive missions against the preplanned missions. The dynamics model for Bluefin AUVs was used for all AUVs in all virtual experiments in order to keep the AUV dynamics constant. In all cases, Unicorn and Macrura were the two AUVs selected to run mission in a single virtual experiment. In the one set of virtual experiments that includes the front-following preplanned zigzag mission in addition to the adaptive zigzag and straight preplanned zigzag missions, Neptune is used as the third AUV. The array of virtual experiments and their configurations, goals, and missions for each AUV are summarized below and detailed in Tables 5.3, 5.4, \& 5.5.

- Runs 0-7: 2D, 2-AUV, 1 adaptive \& 1 preplanned, vary adaptive and straight zigzag amplitude (8 virtual experiments)

- Runs 8-11: 2D, 2-AUV, both adaptive, comparative: follow-the-leader \& rubber band (4 virtual experiments)

- Runs 12-13: 2D, 3-AUV, comparative: 1 adaptive, 1 preplanned straight, \& 1 preplanned along-front zigzag (2 virtual experiments)

- Runs 14-21: 2D, 2-AUV, 1 adaptive \& 1 preplanned, vary detection-phase loiter radii (8 virtual experiments)

- Runs 22-23: 2D, 2-AUV, both adaptive, compare: detection-phase loiter \& detection-phase spiral (2 virtual experiments)

- Runs 24-31: 3D, 2-AUV, 1 adaptive zigzag \& 1 adaptive helix, vary depth $\&$ helix dimensions (4 virtual experiments)

- Runs 32-33: 2D, 2-AUV, both adaptive, compare AW06 \& SW06 model resolution effects (2 virtual experiments)

- Runs 36-64: 2D, 2-AUV, 1 adaptive \& 1 preplanned, constant amplitude and depth, comparative: adaptive $\&$ preplanned straight zigzag (29 virtual experiments) 
Table 5.3: Virtual Experiment Configurations: Runs 0-7 \& 8-11

\begin{tabular}{|c|c|c|c|c|}
\hline \multicolumn{2}{|l|}{ Overall Mission } & 2D, vary zigzag amplitudes & $\begin{array}{l}\text { 2D, both adaptive, following, } \\
\text { follow-the-leader vs. rubber } \\
\text { band }\end{array}$ & $\begin{array}{l}\text { 2D, both adaptive, following, } \\
\text { follow-the-leader vs. rubber } \\
\text { band }\end{array}$ \\
\hline \multicolumn{2}{|l|}{ Run ID } & 0-7 & 8,10 & 9,11 \\
\hline \multicolumn{2}{|l|}{ Unicorn Mission } & BHV_FrontTrackNoBdry & $\begin{array}{l}\text { BHV_FrontTrackNoBdry, } \\
\text { BHV_FollowTheLeader }\end{array}$ & BHV_FrontTrackNoBdry \\
\hline \multicolumn{2}{|l|}{ Macrura Mission } & $\begin{array}{l}\text { Straight zigzag } \\
\text { (BHV_Waypoint) }\end{array}$ & $\begin{array}{l}\text { BHV_FrontTrackNoBdry, } \\
\text { BHV_FollowTheLeader }\end{array}$ & $\begin{array}{l}\text { BHV_FrontTrackNoBdry, } \\
\text { BHV_RubberBand }\end{array}$ \\
\hline \multicolumn{2}{|l|}{ Neptune Mission } & none & none & none \\
\hline \multirow{3}{*}{$\begin{array}{l}\text { Adaptive Front } \\
\text { Track Zigzag } \\
\text { Config }\end{array}$} & Loiter/Spiral & loiter & loiter & loiter \\
\hline & Loiter Radius [m] & 2000 & 2000 & 2000 \\
\hline & $\begin{array}{l}\text { Zigzag Amplitude } \\
\text { [m] }\end{array}$ & $100,500,1000,5000$ & 500 & 500 \\
\hline \multirow{4}{*}{$\begin{array}{l}\text { Adaptive Front } \\
\text { Track Helix } \\
\text { Config }\end{array}$} & Loiter/Spiral & - & - & - \\
\hline & Loiter Radius [m] & - & - & - \\
\hline & Helix Radius [m] & - & - & - \\
\hline & $\begin{array}{l}\text { Helix Constant } \\
{[\mathrm{m}]}\end{array}$ & - & - & - \\
\hline \multirow{5}{*}{$\begin{array}{l}\text { Preplanned } \\
\text { Straight Zigzag } \\
\text { Config }\end{array}$} & Zigzag Width [m] & $200,1000,2000,10000$ & - & - \\
\hline & Zigzag Length [m] & 60000 & - & - \\
\hline & Zigzag Period [m] & $400,2000,4000,20000$ & - & - \\
\hline & $\begin{array}{l}\text { Zigzag Amplitude } \\
\text { [m] }\end{array}$ & $100,500,1000,5000$ & - & - \\
\hline & $\begin{array}{l}\text { Zigzag Heading } \\
{\left[{ }^{\circ}\right]}\end{array}$ & 109 & - & - \\
\hline \multicolumn{2}{|c|}{$\begin{array}{l}\text { Preplanned Front-Following Zigzag } \\
\text { Config }\end{array}$} & - & - & - \\
\hline \multirow{2}{*}{$\begin{array}{l}\text { Follow-the- Leader } \\
\text { Config }\end{array}$} & Leader & - & Unicorn & - \\
\hline & Follower & - & Macrura & - \\
\hline \multicolumn{2}{|c|}{ Rubber Band Config } & - & - & $\begin{array}{l}\text { Station Point }=\text { Unis } \mathrm{x}-\mathrm{y} \\
\text { position; Inner Radius }=5 \mathrm{~km} \text {; } \\
\text { Outer Radius }=10 \mathrm{~km} \text {; Outer } \\
\text { Speed }=1.8 \mathrm{~m} / \mathrm{s} \text {; Stiffness }=0.1\end{array}$ \\
\hline \multicolumn{2}{|l|}{ Model } & SW06 & SW06 & SW06 \\
\hline \multicolumn{2}{|c|}{ Depth/Helix Center Depth [m] } & 30 & 30 & 30 \\
\hline \multicolumn{2}{|c|}{ Start Time(s) } & $\begin{array}{l}\text { 08/28/2006 12:00:00 GMT \& } \\
\text { 09/03/2006 12:00:00 GMT }\end{array}$ & $\begin{array}{l}\text { 08/28/2006 12:00:00 GMT \& } \\
\text { 09/03/2006 12:00:00 GMT }\end{array}$ & $\begin{array}{l}\text { 08/28/200612:00:00 GMT \& } \\
\text { 09/03/2006 12:00:00 GMT }\end{array}$ \\
\hline \multicolumn{2}{|c|}{$\begin{array}{l}\text { Datum (SW corner of } 200 \mathrm{~km} \times 200 \\
\text { km Op Box) }\end{array}$} & $\left(38.6^{\circ} \mathrm{N},-71.9^{\circ} \mathrm{E}\right)$ & $\left(38.6^{\circ} \mathrm{N},-71.9^{\circ} \mathrm{E}\right)$ & $\left(38.6^{\circ} \mathrm{N},-71.9^{\circ} \mathrm{E}\right)$ \\
\hline \multicolumn{2}{|c|}{ Start Location $([\mathbf{m}],[\mathrm{m}])$} & $(100000,113000)$ & $(100000,113000)$ & $(100000,113000)$ \\
\hline \multicolumn{2}{|c|}{ Desired Speed $[\mathrm{m} / \mathrm{s}]$} & 2 & 2 & 2 \\
\hline
\end{tabular}

$-=$ Configuration not applicable during the associated runs. 
Table 5.4: Virtual Experiment Configurations: Runs 12-13, 14-21, \& 22-23

\begin{tabular}{|c|c|c|c|c|}
\hline \multicolumn{2}{|l|}{ Overall Mission } & 2D, 3-AUV comparison & $2 \mathrm{D}$, vary adaptive loiter radius & 2D, detection loiter vs. spiral \\
\hline \multicolumn{2}{|l|}{ Run ID } & $12-13$ & $14-21$ & $22-23$ \\
\hline \multicolumn{2}{|l|}{ Unicorn Mission } & BHV_FrontTrackNoBdry & BHV_FrontTrackNoBdry & BHV_FrontTrackNoBdry \\
\hline \multicolumn{2}{|l|}{ Macrura Mission } & $\begin{array}{l}\text { Straight zigzag } \\
\text { (BHV_Waypoint) }\end{array}$ & $\begin{array}{l}\text { Straight zigzag } \\
\text { (BHV_Waypoint) }\end{array}$ & BHV_FrontTrackNoBdry \\
\hline \multicolumn{2}{|l|}{ Neptune Mission } & $\begin{array}{l}\text { Front-following zigzag } \\
\text { (BHV_Waypoint) }\end{array}$ & none & none \\
\hline \multirow{3}{*}{$\begin{array}{l}\text { Adaptive Front } \\
\text { Track Zigzag } \\
\text { Config }\end{array}$} & Loiter/Spiral & loiter & loiter & loiter (Unicorn), spiral (Macrura) \\
\hline & Loiter Radius [m] & 2000 & $1000,2000,4000,8000$ & 2000 \\
\hline & $\begin{array}{l}\text { Zigzag Amplitude } \\
{[\mathrm{m}]}\end{array}$ & 500 & 500 & 500 \\
\hline \multirow{4}{*}{$\begin{array}{l}\text { Adaptive Front } \\
\text { Track Helix } \\
\text { Config }\end{array}$} & Loiter/Spiral & - & - & - \\
\hline & Loiter Radius [m] & - & - & - \\
\hline & Helix Radius [m] & - & 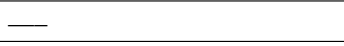 & - \\
\hline & $\begin{array}{l}\text { Helix Constant } \\
{[\mathrm{m}]}\end{array}$ & - & - & - \\
\hline \multirow{5}{*}{$\begin{array}{l}\text { Preplanned } \\
\text { Straight Zigzag } \\
\text { Config }\end{array}$} & Zigzag Width [m] & 8000 & 8000 & 8000 \\
\hline & Zigzag Length [m] & 60000 & 60000 & 60000 \\
\hline & Zigzag Period [m] & 16000 & 16000 & 16000 \\
\hline & $\begin{array}{l}\text { Zigzag Amplitude } \\
{[\mathrm{m}]}\end{array}$ & 4000 & 4000 & 4000 \\
\hline & $\begin{array}{l}\text { Zigzag Heading } \\
{\left[{ }^{\circ}\right]}\end{array}$ & 101 & 109 & $109 \& 101$ \\
\hline \multicolumn{2}{|c|}{$\begin{array}{l}\text { Preplanned Front-Following Zigzag } \\
\text { Config }\end{array}$} & $\begin{array}{l}\text { Selected waypoints (see Table } \\
5.10 \text { ) }\end{array}$ & - & - \\
\hline \multirow{2}{*}{$\begin{array}{l}\text { Follow-the- Leader } \\
\text { Config }\end{array}$} & Leader & - & - & - \\
\hline & Follower & - & - & - \\
\hline \multicolumn{2}{|c|}{ Rubber Band Config } & - & - & - \\
\hline \multicolumn{2}{|c|}{ Model } & SW06 & SW06 & SW06 \\
\hline \multicolumn{2}{|c|}{ Depth/Helix Center Depth [m] } & 30 & 30 & 30 \\
\hline \multicolumn{2}{|l|}{ Start Time(s) } & $\begin{array}{l}\text { 08/28/2006 12:00:00 GMT \& } \\
\text { 09/03/2006 12:00:00 GMT }\end{array}$ & $\begin{array}{l}\text { 08/28/2006 12:00:00 GMT \& } \\
\text { 09/03/2006 12:00:00 GMT }\end{array}$ & $\begin{array}{l}\text { 08/28/2006 12:00:00 GMT \& } \\
\text { 09/03/2006 12:00:00 GMT }\end{array}$ \\
\hline \multicolumn{2}{|c|}{$\begin{array}{l}\text { Datum (SW corner of } 200 \mathrm{~km} \times 200 \\
\mathrm{~km} \text { Op Box) }\end{array}$} & $\left(39.409291^{\circ} \mathrm{N},-71.934359^{\circ} \mathrm{E}\right)$ & $\left(38.6^{\circ} \mathrm{N},-71.9^{\circ} \mathrm{E}\right)$ & $\begin{array}{l}\left(38.6^{\circ} \mathrm{N},-71.9^{\circ} \mathrm{E}\right) \& \\
\left(39.409291^{\circ} \mathrm{N},-71.934359^{\circ} \mathrm{E}\right)\end{array}$ \\
\hline \multicolumn{2}{|c|}{ Start Location $([\mathbf{m}],[\mathbf{m}])$} & $(100000,113000)$ & $(100000,113000)$ & $(100000,113000)$ \\
\hline \multicolumn{2}{|c|}{ Desired Speed $[\mathrm{m} / \mathrm{s}]$} & 2 & 2 & 2 \\
\hline
\end{tabular}

- $=$ Configuration not applicable during the associated runs. 
Table 5.5: Virtual Experiment Configurations: Runs 24-31, 32-33, \& 36-64

\begin{tabular}{|c|c|c|c|c|}
\hline \multicolumn{2}{|l|}{ Overall Mission } & $\begin{array}{l}\text { 3D, adaptive zigzag vs. } \\
\text { adaptive helix }\end{array}$ & $\begin{array}{c}2 \mathrm{D} \text {, adaptive zigzag in } 3 \mathrm{~km} \text { vs. } \\
1 \mathrm{~km} \text { resolution environment, } \\
\text { AW06 domain }\end{array}$ & $\begin{array}{l}\text { 2D, adaptive vs. preplanned } \\
\text { straight zigzag, random start } \\
\text { times }\end{array}$ \\
\hline \multicolumn{2}{|l|}{ Run ID } & $24-31$ & $32-33$ & $36-64$ \\
\hline \multicolumn{2}{|l|}{ Unicorn Mission } & BHV_FrontTrackNoBdry & BHV_FrontTrackNoBdry & BHV_FrontTrackNoBdry \\
\hline \multicolumn{2}{|l|}{ Macrura Mission } & BHV_FrontTrackHelix & BHV_FrontTrackNoBdry & $\begin{array}{l}\text { Straight zigzag } \\
\text { (BHV_Waypoint) }\end{array}$ \\
\hline \multicolumn{2}{|l|}{ Neptune Mission } & none & none & none \\
\hline \multirow{3}{*}{$\begin{array}{l}\text { Adaptive Front } \\
\text { Track Zigzag } \\
\text { Config }\end{array}$} & Loiter/Spiral & loiter & loiter & loiter \\
\hline & Loiter Radius [m] & 2000 & 2000 & 2000 \\
\hline & $\begin{array}{l}\text { Zigzag Amplitude } \\
\text { [m] }\end{array}$ & 500 & 500 & 500 \\
\hline \multirow{4}{*}{$\begin{array}{l}\text { Adaptive Front } \\
\text { Track Helix } \\
\text { Config }\end{array}$} & Loiter/Spiral & loiter & - & - \\
\hline & Loiter Radius [m] & 2000 & - & - \\
\hline & Helix Radius [m] & $30,100,300,1000$ & - & - \\
\hline & $\begin{array}{l}\text { Helix Constant } \\
{[\mathrm{m}]}\end{array}$ & $60,200,600,2000$ & - & - \\
\hline \multirow{5}{*}{$\begin{array}{l}\text { Preplanned } \\
\text { Straight Zigzag } \\
\text { Config }\end{array}$} & Zigzag Width [m] & - & - & 8000 \\
\hline & Zigzag Length [m] & - & - & 60000 \\
\hline & Zigzag Period [m] & - & - & 16000 \\
\hline & $\begin{array}{l}\text { Zigzag Amplitude } \\
{[\mathrm{m}]}\end{array}$ & - & - & 4000 \\
\hline & $\begin{array}{l}\text { Zigzag Heading } \\
{\left[{ }^{\circ}\right]}\end{array}$ & - & - & $109 \& 101$ \\
\hline \multicolumn{2}{|c|}{$\begin{array}{l}\text { Preplanned Front-Following Zigzag } \\
\text { Config }\end{array}$} & - & - & - \\
\hline \multirow{2}{*}{$\begin{array}{l}\text { Follow-the- Leader } \\
\text { Config }\end{array}$} & Leader & - & - & - \\
\hline & Follower & - & - & - \\
\hline \multicolumn{2}{|c|}{ Rubber Band Config } & - & - & - \\
\hline \multicolumn{2}{|c|}{ Model } & SW06 & $\begin{array}{l}\text { SW06 (Macrura), AW06 } \\
\text { (Unicron) }\end{array}$ & SW06 \\
\hline \multicolumn{2}{|c|}{ Depth/Helix Center Depth [m] } & $30,100,300,1000$ & 30 & 30 \\
\hline \multicolumn{2}{|c|}{ Start Time(s) } & $\begin{array}{l}\text { 08/28/2006 12:00:00 GMT \& } \\
\text { 09/03/2006 12:00:00 GMT }\end{array}$ & $\begin{array}{l}\text { 08/28/2006 12:00:00 GMT \& } \\
\text { 09/03/2006 12:00:00 GMT }\end{array}$ & $\begin{array}{l}\text { Psudo-random, between } \\
08 / 28 / 2006 \& 09 / 08 / 2006\end{array}$ \\
\hline \multicolumn{2}{|c|}{$\begin{array}{l}\text { Datum (SW corner of } 200 \mathrm{~km} \times 200 \\
\text { km Op Box) }\end{array}$} & $\left(39.409291^{\circ} \mathrm{N},-71.934359^{\circ} \mathrm{E}\right)$ & $\left(38.4^{\circ} \mathrm{N},-73.4^{\circ} \mathrm{E}\right)$ & $\begin{array}{l}\left(38.6^{\circ} \mathrm{N},-71.9^{\circ} \mathrm{E}\right) \& \\
\left(39.409291^{\circ} \mathrm{N},-71.934359^{\circ} \mathrm{E}\right)\end{array}$ \\
\hline \multicolumn{2}{|c|}{ Start Location $([\mathrm{m}],[\mathrm{m}])$} & $(100000,25000)$ & $(100000,113000)$ & $(100000,113000)$ \\
\hline \multicolumn{2}{|c|}{ Desired Speed $[\mathrm{m} / \mathrm{s}]$} & 2 & 2 & 2 \\
\hline
\end{tabular}

$-=$ Configuration not applicable during the associated runs. 


\subsection{Analysis}

All virtual experiment runs listed at the end of Section 5.7.4 will be qualitatively and quantitatively analyzed here using a number of performance metrics and observations of the missions' performance. For the large batch of 2-AUV virtual experiments comparing the $2 \mathrm{D}$ adaptive front tracking behavior to the pre-planned straight zigzag behavior (Runs 36-64, Section 5.7.4), the overall improvement (or lack thereof) of the adaptive missions over the preplanned ones will also be quantified.

\subsubsection{Performance Metrics}

A number of performance metrics have been developed to evaluate and compare the front sampling ability of both preplanned and adaptive AUV front tracking missions. The variables measured during the front tracking missions to calculate the performance metrics are given below, followed by the calculations for the metrics themselves.

\section{Variables}

$t_{\text {mission }}=$ Total mission time

$v_{\text {avg }}=$ Average AUV speed

$v_{\text {nav }}=$ Actual AUV speed at a given time (sample)

$N_{s p d}=$ Total number of AUV speed sample points

$$
v_{a v g}=\frac{1}{N_{s p d}} \sum_{i=1}^{N_{s p d}}\left(v_{\text {nav }}\right)_{i}
$$

$\theta=$ Front intersect angle

$D_{\text {total }}=$ Total distance traveled

$$
D_{\text {total }}=\sum_{i=1}^{N_{\text {pos }}} \sqrt{\left(x_{i}-x_{i-1}\right)^{2}+\left(y_{i}-y_{i-1}\right)^{2}}
$$

where $N_{\text {pos }}=$ Total number of AUV position locations

$D_{\text {front }}=$ Total possible distance AUV could have tracked along front, given $t_{\text {mission }}$. Estimated by the bestcase calculation (AUV perfectly tracks the front, crossing the front at an angle of $\theta$ ).

$$
D_{\text {front }}=t_{\text {mission }} v_{\text {avg }} \cos (\theta)
$$


$D_{\text {on_front }}=$ Distance AUV tracked along the front line $\left(\leq D_{\text {front }}\right)$

$N_{\text {cross }}=$ Number of front crossing points, total, while tracking front

$D_{\text {from_front }}=$ Perpendicular distance (closest point of approach) from AUV position, $(x, y)$, to front estimate line

$$
D_{\text {from_front }}=\frac{-m x+y-b}{\sqrt{\left(m^{2}+1\right)}}
$$

where $m$ and $b$ are the slope and intercept, respectively, of the front line estimate in the local $\mathrm{x}-\mathrm{y}$ grid $\partial T / \partial r=$ The temperature $(T)$ gradient in the across-front direction, relative to the front estimate line

$$
\frac{\partial T}{\partial r}=\frac{\partial T}{\partial D_{\text {from_front }}}
$$

\section{Metrics}

$\rho=$ Crossing Density; i.e., how many front crossings were made by the AUV per unit length of the front line that was tracked (higher values equal better performance)

$$
\rho=\frac{N_{\text {cross }}}{D_{\text {on_front }}}
$$

$D_{\text {cross }}=$ Distance between Crossings; i.e., the average distance the AUV traveled between front crossings (higher values equal worse performance)

$$
D_{\text {cross }}=\frac{1}{\rho}
$$

$\epsilon=$ Front Sampling Efficiency; i.e., the percentage of $D_{\text {front }}$ that was tracked and sampled by the AUV (higher values equal better performance)

$$
\epsilon=\frac{D_{\text {on_front }}}{D_{\text {front }}} \times 100 \%
$$

$E R=$ Excess Ratio; i.e., how much of the AUV's travel distance was in excess of the distance along the front that the AUV captured the front (higher values equal worse performance)

$$
E R=\frac{D_{\text {total }}}{D_{\text {on_front }}}
$$


$F E E=$ Front Estimate Error, which compares the $|\partial T / \partial r|_{\max }$ location to the local estimated front location, as captured by the AUV (higher values equal worse performance)

$$
F E E=D_{\text {from_front }} @|\partial T / \partial r|_{\max } \text { on a zigzag leg }
$$

$T C=$ Tracking Confidence, which is an evaluation of the confidence level that the actual front was followed/sampled by the AUV, expressed as a percentage (higher values equal better performance)

$$
T C=2\left(\frac{N^{+}}{N_{t o t}}\right) \times 100 \%
$$

where $N^{+}$is the number of above-average $\partial T / \partial r$ bins, $N_{\text {tot }}$ is the total number of $\partial T / \partial r$ bins, and the scaling factor of 2 accounts for the fact that most $\partial T / \partial r$ sample bins have below-average values, and a minority of samples have above-average values due to sharp peaks in $\partial T / \partial r$ in the across-front direction, so at best it would be expected to see $N^{+} / N_{\text {tot }}=0.5$.

In order to determine the distance each AUV traveled along the front, $D_{\text {on_front }}$, each virtual experiment run had to be replayed twice in post-processing using the Google Earth interface for Ocean Vehicles (GEOV) with auto-updating overlays of the temperature field showing highlighted isothermal lines at the AUV-calculated frontal temperature. For each replay, Google Earth's Path tool was used to track the path of one AUV along the frontal isotherm, discontinuing and resuming the path segments when the AUV strayed from and reacquired the front (respectively). The sum of the path segment lengths from a single replay was recorded as $D_{\text {on_front }}$ for the AUV whose path was mapped. All other performance metrics variables that did not involve $D_{\text {on_front }}$ for calculation were actively recorded and updated as the virtual experiments were running. The MOOS process pFrontTrackMetrics was written to keep track of the performance metrics variables' values as the virtual experiments ran.

Once all of the performance metrics variables' values were extracted from the data logs and $D_{\text {on_front }}$ values were determined, the actual performance metrics were calculated. When performing the data extraction, only data collected on both AUVs while the preplanned straight zigzag was being executed were used. In the virtual experiments where both AUVs were adaptively tracking the front, a timer was set to stop both AUVs' missions simultaneously to maintain equal mission time. This allows us to keep the total mission time for both AUVs approximately equal to improve validity of comparison of the two front mapping techniques (preplanned vs. adaptive) and evaluation of adaptive front tracking configuration variables.

Based on the performance metrics defined in Equations 5.21-5.24 and summarized in Table 5.15 at the 
end of this chapter, higher Crossing Density $(\rho)$ and Sampling Efficiency $(\epsilon)$ values indicate better performance tracking along the front, while higher Distance between Crossings $\left(D_{\text {cross }}\right)$ and Excess Ratio $(E R)$ values indicate worse performance. It is often desirable to maintain some across-front motion of the AUVs as well, thus extremes of huge $\rho$ and tiny $D_{\text {cross }}$ and $E R$ values are not necessarily optimal.

In summary, sailing along the front is good, but in many cases, crossing the front frequently is also good. The desirable performance metrics criteria for these two cases are summarized here:

- Sailing very closely along the front should maximize Crossing Density and Sampling Efficiency and minimize Distance between Crossings and Excess Ratio as much as possible.

- Maintaining crossing of the front over some length scale-i.e., the Rossby radius of deformation (e.g., 3-10 km) — while sailing along the front should maximize Sampling Efficiency and result in relatively high Crossing Density and low Distance between Crossings and Excess Ratio (but not the extreme values desirable for directly along-front motion).

\subsubsection{Data Analysis}

\section{Runs 36-64: 2D, comparing adaptive \& preplanned straight zigzag front tracking}

These 2D, 2-AUV (1 adaptive, 1 preplanned) virtual experiments maintained constant AUV zigzag amplitudes and operational depth, aiming to collect data for a comparison of adaptive zigzag and preplanned straight zigzag front tracking methods.

Virtual experiment Runs 36-64 were designed to determine a baseline of performance for adaptive front tracking versus preplanned straight zigzag front sampling. A quantitative analysis of preplanned versus adaptive missions has been performed for the case of single AUVs doing 2D front tracking. The Unicorn AUV was tasked with 2D adaptive front tracking, while the Macrura AUV was given a preplanned fixed zigzag pattern to execute. In all cases, both Unicorn (adaptive) and Macrura (preplanned) started at the same location and executed their paths over the same mission duration. Macrura's preplanned path was selected to cover the general location of the front near the MAB shelfbreak south of Cape Cod, MA. The preplanned path's configuration is given in Table 5.6. Unicorn's adaptive zigzag was configured based on the spatiotemporal scales of the front and the speed limitations of the AUV. The adaptive front tracking configuration parameters are given in Table 5.7.

With this setup, 29 missions were completed with both AUVs starting at (100000 m, $113000 \mathrm{~m})$ relative to two Datum locations: $\left(38.6^{\circ} \mathrm{N},-71.9^{\circ} \mathrm{E}\right)$ and $\left(39.409291^{\circ} \mathrm{N},-71.934359^{\circ} \mathrm{E}\right)$. The former (more southerly, 
Table 5.6: Configuration of Macrura's Preplanned Straight Zigzag Path

\begin{tabular}{|l|l|}
\hline Characteristic & Value \\
\hline \hline Start Location (local XY grid) & $(100000 \mathrm{~m}, 113000 \mathrm{~m})$ \\
\hline Datum (old/new) & $\begin{array}{l}\left(38.6^{\circ} \mathrm{N},-71.9^{\circ} \mathrm{E}\right) /\left(39.409291^{\circ} \mathrm{N},\right. \\
\left.-71.934359^{\circ} \mathrm{E}\right)\end{array}$ \\
\hline Heading (old/new) & $109^{\circ} / 101^{\circ}$ \\
\hline Survey Length & $60000 \mathrm{~m}$ \\
\hline Survey Width & $8000 \mathrm{~m}$ \\
\hline Survey Period & $16000 \mathrm{~m}$ \\
\hline
\end{tabular}

Table 5.7: Configuration of Unicorn's Adaptive Zigzag Path

\begin{tabular}{|l|l|}
\hline Characteristic & Value \\
\hline \hline Start Location (local XY grid) & $(100000 \mathrm{~m}, 113000 \mathrm{~m})$ \\
\hline Datum (old/new) & $\begin{array}{l}\left(38.6^{\circ} \mathrm{N},-71.9^{\circ} \mathrm{E}\right) /\left(39.409291^{\circ} \mathrm{N},\right. \\
\left.-71.934359^{\circ} \mathrm{E}\right)\end{array}$ \\
\hline Front's Spatial Scale & $10000 \mathrm{~m}$ \\
\hline Front's Temporal Scale & $36000 \mathrm{~s}$ \\
\hline zigzag Amplitude & $500 \mathrm{~m}$ \\
\hline Time Between Re-Initializations & $36000 \mathrm{~s}$ \\
\hline Front Intersect Angle & $45^{\circ}$ \\
\hline Initial Pattern & Loiter, radius $2000 \mathrm{~m}$ \\
\hline
\end{tabular}

'old') Datum location corresponded with a fixed zigzag heading of $109^{\circ}$, while the latter ('new') Datum required a fixed zigzag heading of $101^{\circ}$ to accommodate the slight change in general heading of the front north of the shelfbreak. The various performance metric variables were tracked while the virtual experiments were running and, after the fact, the performance metrics themselves were calculated and plotted for both AUVs in post-processing. These results are shown in Figs. 5-16, 5-17, and 5-18, where each figure represents a different set of virtual experiment runs. In order to keep as many parameters constant as possible, the AUV running the preplanned zigzag (Macrura) tracked its front crossings based on the frontal temperature that the adaptive AUV (Unicorn) determined for front tracking.

As mentioned previously, based one the performance metrics defined in Equations 5.21-5.24, higher Crossing Density $(\rho)$ and Sampling Efficiency $(\epsilon)$ values indicate better performance, while higher Distance between Crossings $\left(D_{\text {cross }}\right)$ and Excess Ratio $(E R)$ values indicate worse performance. Table 5.8 summarizes the percentage and number of runs in which adaptive front tracking missions were improvements over the 

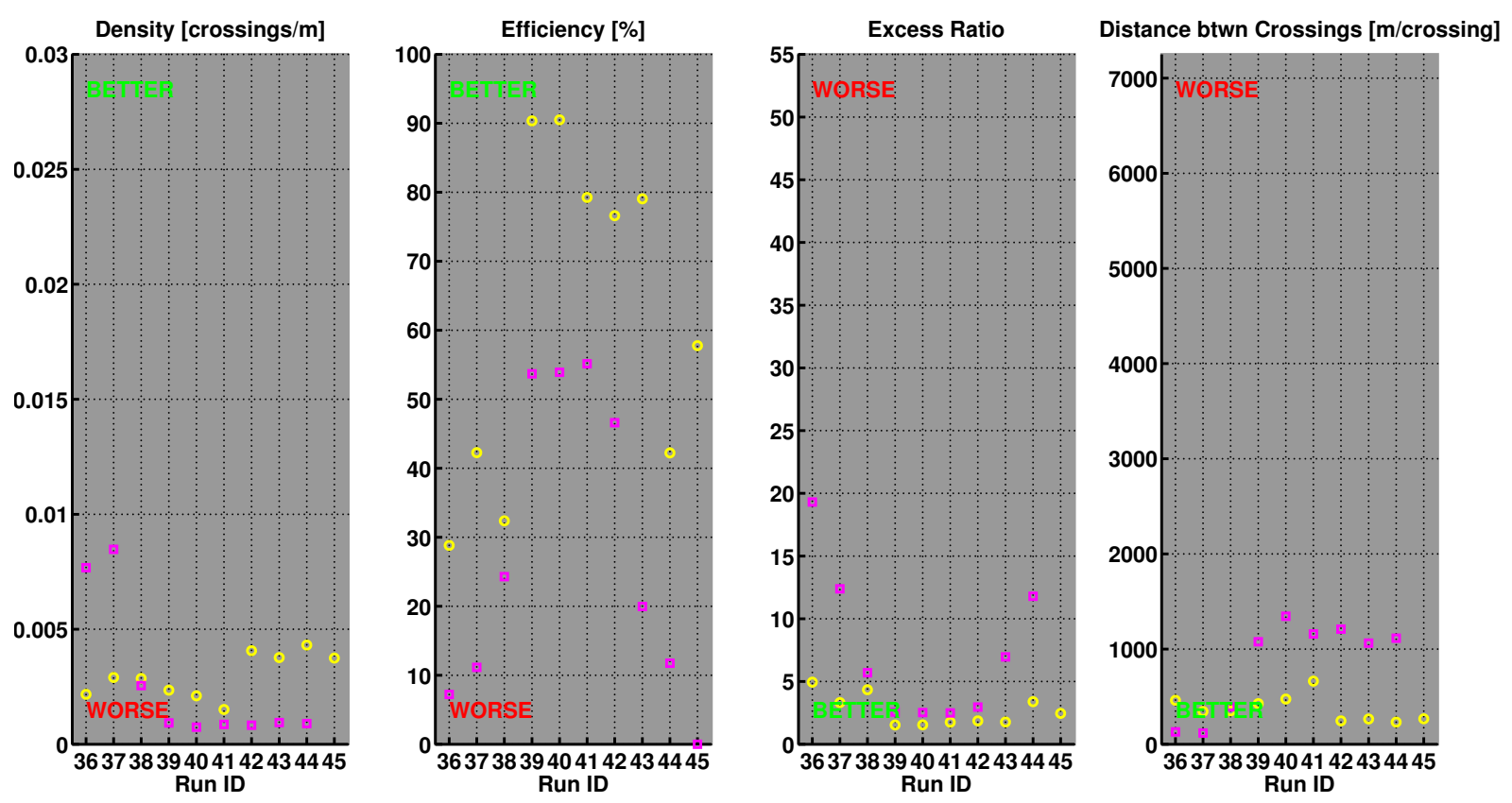

Figure 5-16: Runs 36-45: Performance metrics for Macrura's preplanned zigzag missions (pink squares) and Unicorn's adaptive front tracking missions (yellow circles), plotted for each virtual experiment run. Datum: $\left(38.6^{\circ} \mathrm{N},-71.9^{\circ} \mathrm{E}\right)$. Preplanned zigzag heading: $109^{\circ}$. Better and worse values for each performance metric are indicated on the plots.

preplanned zigzag mission. It is clear from Table 5.8 that, for all performance metrics, the adaptive front tracking algorithms are generally an improvement over using a preplanned zigzag mission for collecting data along a front.

Additionally, Tracking Confidence (TC) was evaluated for the adaptive missions based on temperature data binned (by distance from the front line estimate, into $1 \mathrm{~m}$ bins) over entire missions. The average TC for the adaptive front tracking mission in Runs 36-64 was 59.2\%, with a standard deviation of 9.5\%. The tracking confidence values, along with the Front Estimate Errors (FEE; averaged over the $F E E_{l e g}$ calculated for each leg in a run) for the adaptive front tracking missions in each run are plotted in Figs. 5-19, 5-20, and 5-21. For 28/29 (96.6\%) of the runs, the magnitude of the mean FEE was less than $400 \mathrm{~m}$ (100\% had magnitudes less than $800 \mathrm{~m})$, which is relatively small compared to the $\mathrm{O}(10 \mathrm{~km})$ horizontal spatial scale of the MAB shelfbreak front. The low FEE values mean that the adaptive AUV did a good job covering the actual front interface $\left(|\partial T / \partial r|_{\max }\right)$ while staying close to the estimated front line when tracking the front, and the middling $T C$ values (greater than $40 \%$ for all of the runs, greater than $50 \%$ for $82.8 \%$ the runs, greater than $70 \%$ for $13.8 \%$ of the runs) mean that the adaptive AUV sampled close to the front interface in at least $25 \%$ of the temperature (and thus $\left|\partial T_{\text {binned }} / \partial r\right|$ ) bins in $82.8 \%$ of the runs. 

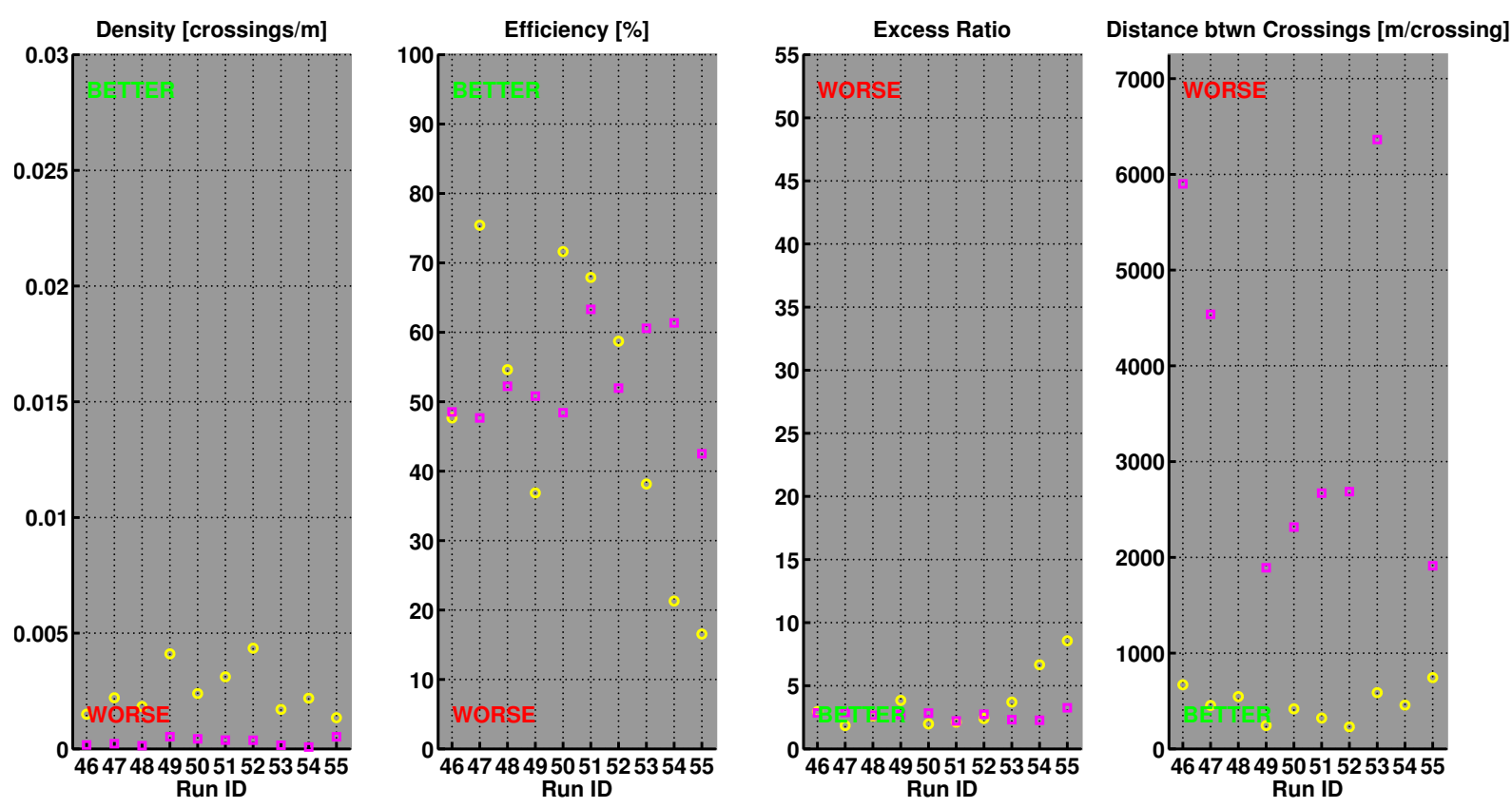

Figure 5-17: Runs 46-55: Performance metrics for Macrura's preplanned zigzag missions (pink squares) and Unicorn's adaptive front tracking missions (yellow circles), plotted for each virtual experiment run. Datum: $\left(39.409291^{\circ} \mathrm{N},-71.934359^{\circ} \mathrm{E}\right)$. Preplanned zigzag heading: $101^{\circ}$. Better and worse values for each performance metric are indicated on the plots.

For the $T C$ and $F E E$ values, it is important to note that the distances represented by these values $(\mathrm{O}(100$ m)) are much smaller than the $3 \mathrm{~km}$ resolution of the ocean model being used for these virtual experiments. Since the data in the model are linearly interpolated between the grid points, it is not fully representative of the smaller scale variations in temperature that would be observed in the real ocean, and thus these $T C$ and $F E E$ values will not be not accurate in real ocean environments (or higher-resolution models). However, these values are accurate for data smoothed or gridded to approximately $3 \mathrm{~km}$ resolution, as was used here. In order to get more accurate $T C$ and FEE results for higher-resolution and real ocean environments, improvements are still needed to the $2 \mathrm{D}$ adaptive front tracking behavior to make it successful in these environments (see analysis from Runs 32-33, below).

In summary, the results and analysis presented above from the comparison of adaptive and preplanned straight zigzag front tracking methods suggest that, for all performance metrics, the use of the adaptive zigzag behavior for front tracking is generally an improvement over using preplanned straight zigzag surveys to collect data along a dynamic ocean front. 

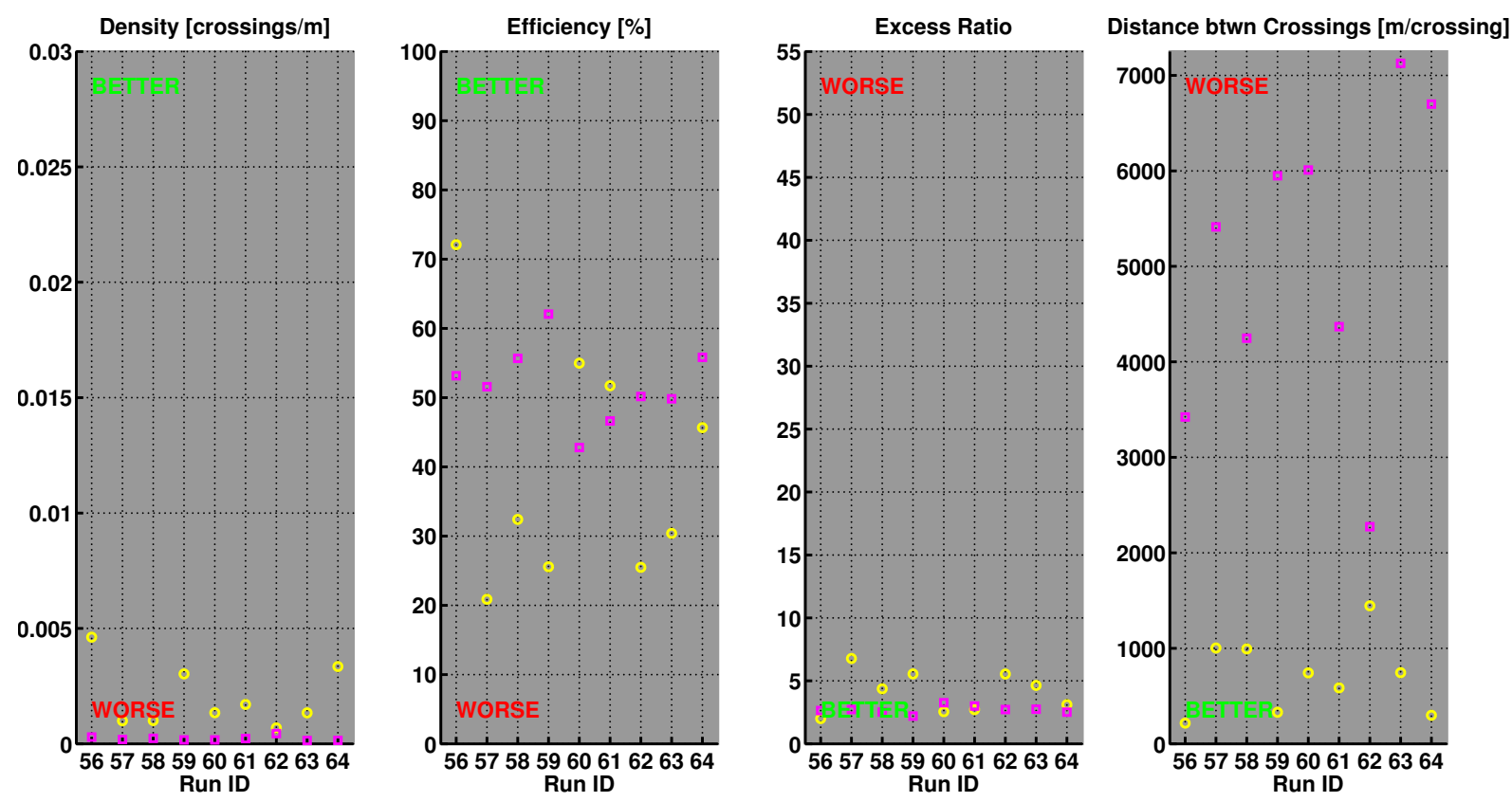

Figure 5-18: Runs 56-64: Performance metrics for Macrura's preplanned zigzag missions (pink squares) and Unicorn's adaptive front tracking missions (yellow circles), plotted for each virtual experiment run. Datum: $\left(39.409291^{\circ} \mathrm{N},-71.934359^{\circ} \mathrm{E}\right)$. Preplanned zigzag heading: $101^{\circ}$. Better and worse values for each performance metric are indicated on the plots.

Table 5.8: Percentage of Runs 36-64 where adaptive missions were improvements over the preplanned zigzag mission, according to the performance metrics.

\begin{tabular}{|c|c|c|c|c|c|c|}
\hline \multirow{2}{*}{ Run IDs } & \multicolumn{2}{|l|}{$36-45$} & \multicolumn{2}{|c|}{$46-64$} & \multicolumn{2}{|l|}{$36-64$} \\
\hline & runs & percent & runs & percent & runs & percent \\
\hline$\rho$ & $8 / 10$ & $80.0 \%$ & $19 / 19$ & $100.0 \%$ & $26 / 29$ & $89.7 \%$ \\
\hline$\epsilon$ & $10 / 10$ & $100.0 \%$ & $8 / 19$ & $42.1 \%$ & $18 / 29$ & $62.1 \%$ \\
\hline$E R$ & $10 / 10$ & $100.0 \%$ & $8 / 19$ & $42.1 \%$ & $17 / 29$ & $58.6 \%$ \\
\hline$D_{\text {cross }}$ & $8 / 10$ & $80.0 \%$ & $19 / 19$ & $100.0 \%$ & $26 / 29$ & $89.7 \%$ \\
\hline Datum/Fixed Zigzag Heading & \multicolumn{2}{|c|}{$\begin{array}{l}\left(38.6^{\circ} \mathrm{N}\right. \\
\left.-71.9^{\circ} \mathrm{E}\right) / 109^{\circ}\end{array}$} & \multicolumn{2}{|c|}{$\begin{array}{l}\left(39.409291^{\circ} \mathrm{N},\right. \\
\left.-71.934359^{\circ} \mathrm{E}\right) / \\
101^{\circ}\end{array}$} & \multicolumn{2}{|l|}{ Total } \\
\hline
\end{tabular}



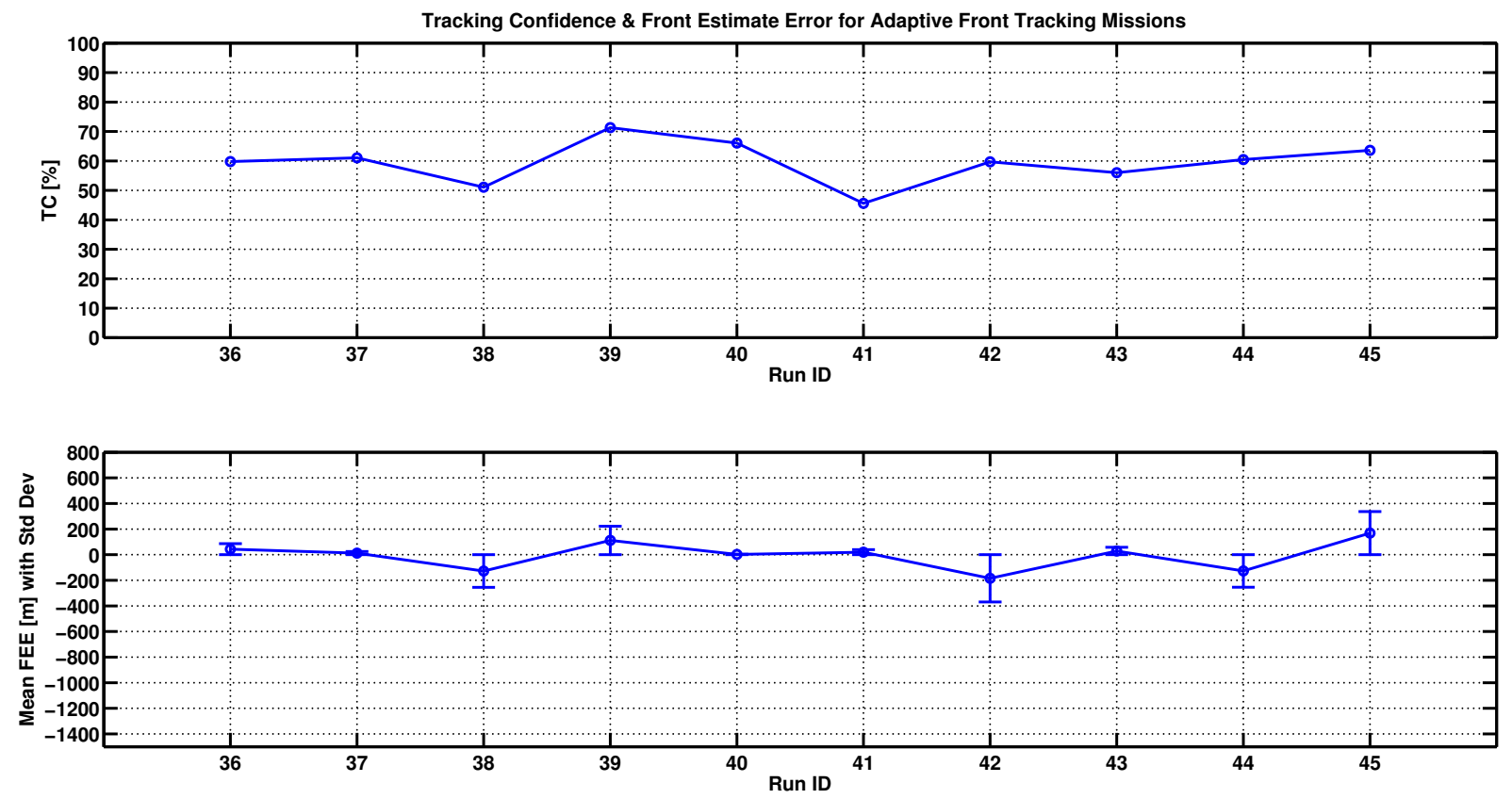

Figure 5-19: Runs 36-45: Tracking Confidence and average Front Estimate Errors for Unicorn's adaptive front tracking missions, plotted for each virtual experiment run. The $T C$ was calculated from spatially-binned temperature data over entire runs. The $F E E$ was averaged over the $F E E_{l e g}$ calculated for each leg in a run, and the standard deviations are plotted here as error bars around the mean values. Datum: $\left(38.6^{\circ} \mathrm{N},-71.9^{\circ} \mathrm{E}\right)$. Better and worse values for each performance metric are indicated on the plots. 

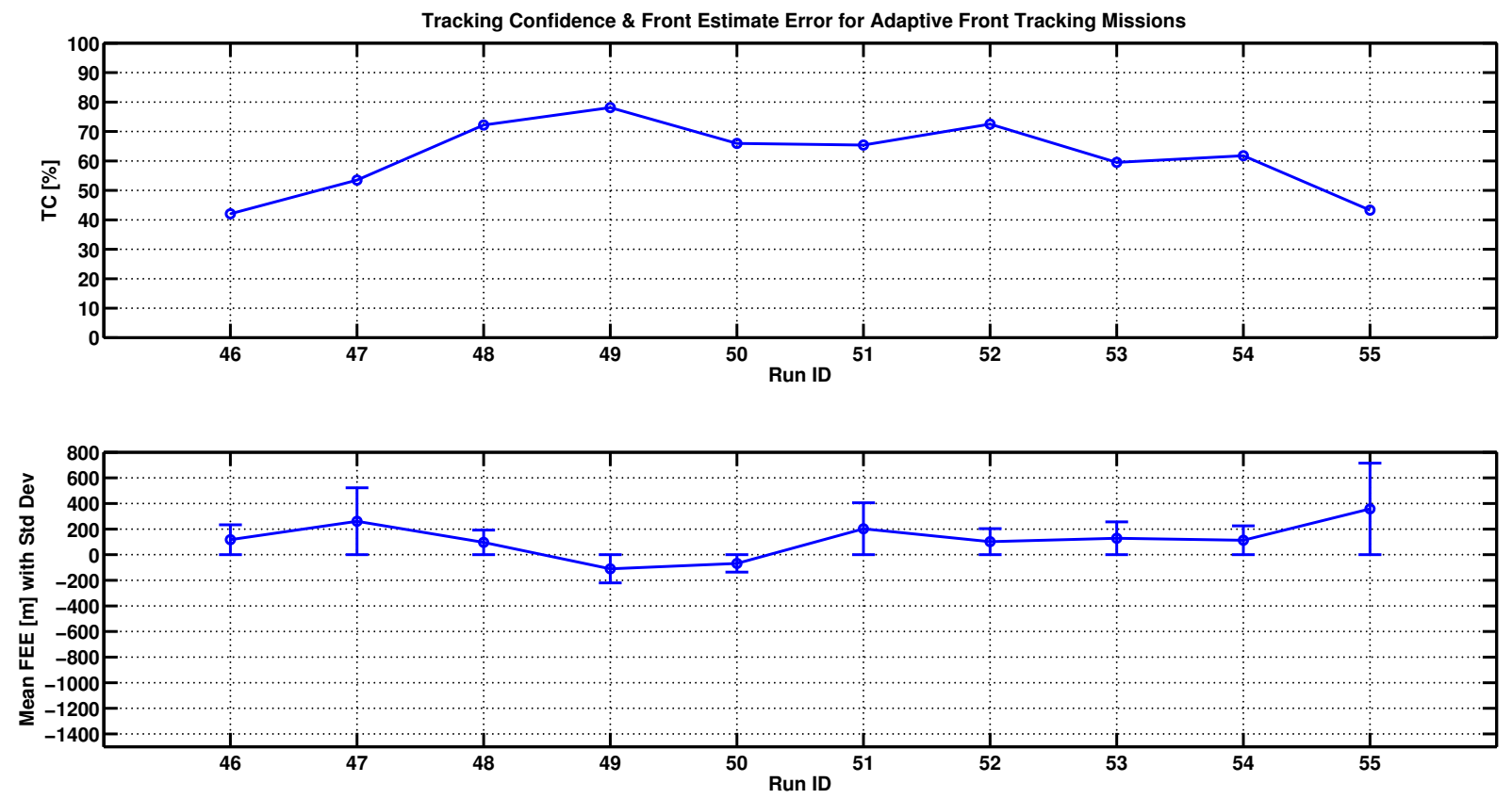

Figure 5-20: Runs 46-55: Tracking Confidence and average Front Estimate Errors for Unicorn's adaptive front tracking missions, plotted for each virtual experiment run. The $T C$ was calculated from spatially-binned temperature data over entire runs. The $F E E$ was averaged over the $F E E_{l e g}$ calculated for each leg in a run, and the standard deviations are plotted here as error bars around the mean values. Datum: $\left(39.409291^{\circ} \mathrm{N}\right.$, $\left.-71.934359^{\circ} \mathrm{E}\right)$. Better and worse values for each performance metric are indicated on the plots. 

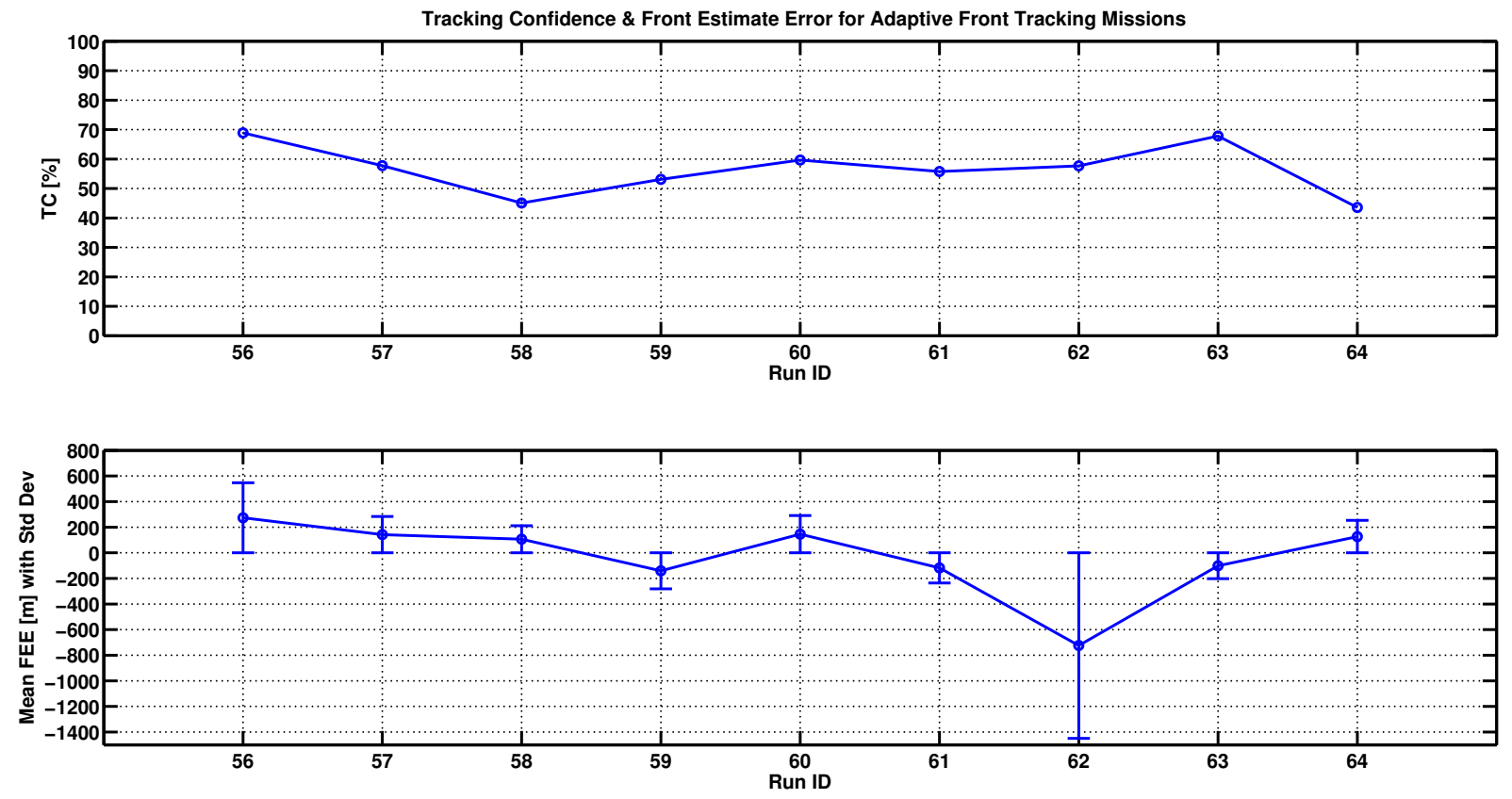

Figure 5-21: Runs 56-64: Tracking Confidence and average Front Estimate Errors for Unicorn's adaptive front tracking missions, plotted for each virtual experiment run. The $T C$ was calculated from spatially-binned temperature data over entire runs. The $F E E$ was averaged over the $F E E_{l e g}$ calculated for each leg in a run, and the standard deviations are plotted here as error bars around the mean values. Datum: $\left(39.409291^{\circ} \mathrm{N}\right.$, $-71.934359^{\circ} \mathrm{E}$ ). Better and worse values for each performance metric are indicated on the plots. 


\section{Runs 0-7: 2D, vary zigzag amplitudes}

These 2D, 2-AUV (1 adaptive, 1 preplanned) virtual experiments maintained constant AUV operational depth while varying the adaptive and straight zigzag amplitudes, aiming to collect data for an evaluation of the effect of zigzag amplitude on the performance of the adaptive and preplanned missions.

Runs $0-7$ evaluated the effect of zigzag amplitude on the preplanned straight zigzag mission and the adaptive front tracking zigzag mission, keeping the zigzag amplitudes and front intersect angles equal between the two missions. The zigzag amplitudes evaluated were $100 \mathrm{~m}$ (Runs 0 \& 4), $500 \mathrm{~m}$ (Runs $1 \& 5$ ), $1000 \mathrm{~m}$ (Runs $2 \&$ 6), and $5000 \mathrm{~m}$ (Runs $3 \& 7$ ). Each zigzag amplitude was evaluated at two model time ranges.

The resulting performance metrics are plotted in Fig. 5-22, and general results are given in Table 5.9. The results that matter most in this case, however, are trends of improvement or degradation in front tracking as the zigzag amplitudes change. Both adaptive and preplanned missions show a general decrease in crossing Density (and increase in Distance between Crossings) as the zigzag amplitude increases, but an increase in Sampling Efficiency. The excess ratio is the most definitive for the preplanned missions, in which an increase in zigzag amplitude corresponds to a sharp decrease in Excess Ratio, suggesting that amplitudes of $\mathrm{O}(1000 \mathrm{~m})$ or more are best for the preplanned straight zigzag. Thus, in the previous and following virtual experiments, a preplanned straight zigzag amplitude of $4000 \mathrm{~m}$ (8000 m zigzag width) was selected. As for the adaptive missions, there is only a slight improvement (decrease) in Excess Ratio as zigzag amplitude increases. Since there is no definitive improvement to using a larger versus smaller adaptive zigzag, a $500 \mathrm{~m}$ amplitude was selected for the baseline adaptive mission.

Table 5.9: Percentage of Runs 0-7 where adaptive missions were improvements over the preplanned zigzag mission, according to the performance metrics.

\begin{tabular}{|l|l|l|}
\hline \multirow{2}{*}{ Run IDs } & \multicolumn{2}{|l|}{$\mathbf{0 - 7}$} \\
\cline { 2 - 3 } & runs & percent \\
\hline \hline$\rho$ & $5 / 8$ & $62.5 \%$ \\
\hline$\epsilon$ & $8 / 8$ & $100 \%$ \\
\hline$E R$ & $8 / 8$ & $100 \%$ \\
\hline$D_{\text {cross }}$ & $5 / 8$ & $62.5 \%$ \\
\hline
\end{tabular}

In summary, the results and analysis presented above from the evaluation of the effect of zigzag amplitude on the performance of the adaptive and preplanned straight zigzag missions suggest that the use of amplitudes of $\mathrm{O}(1000 \mathrm{~m})$ or more are best for the preplanned straight zigzag, while there is no significant increase or 

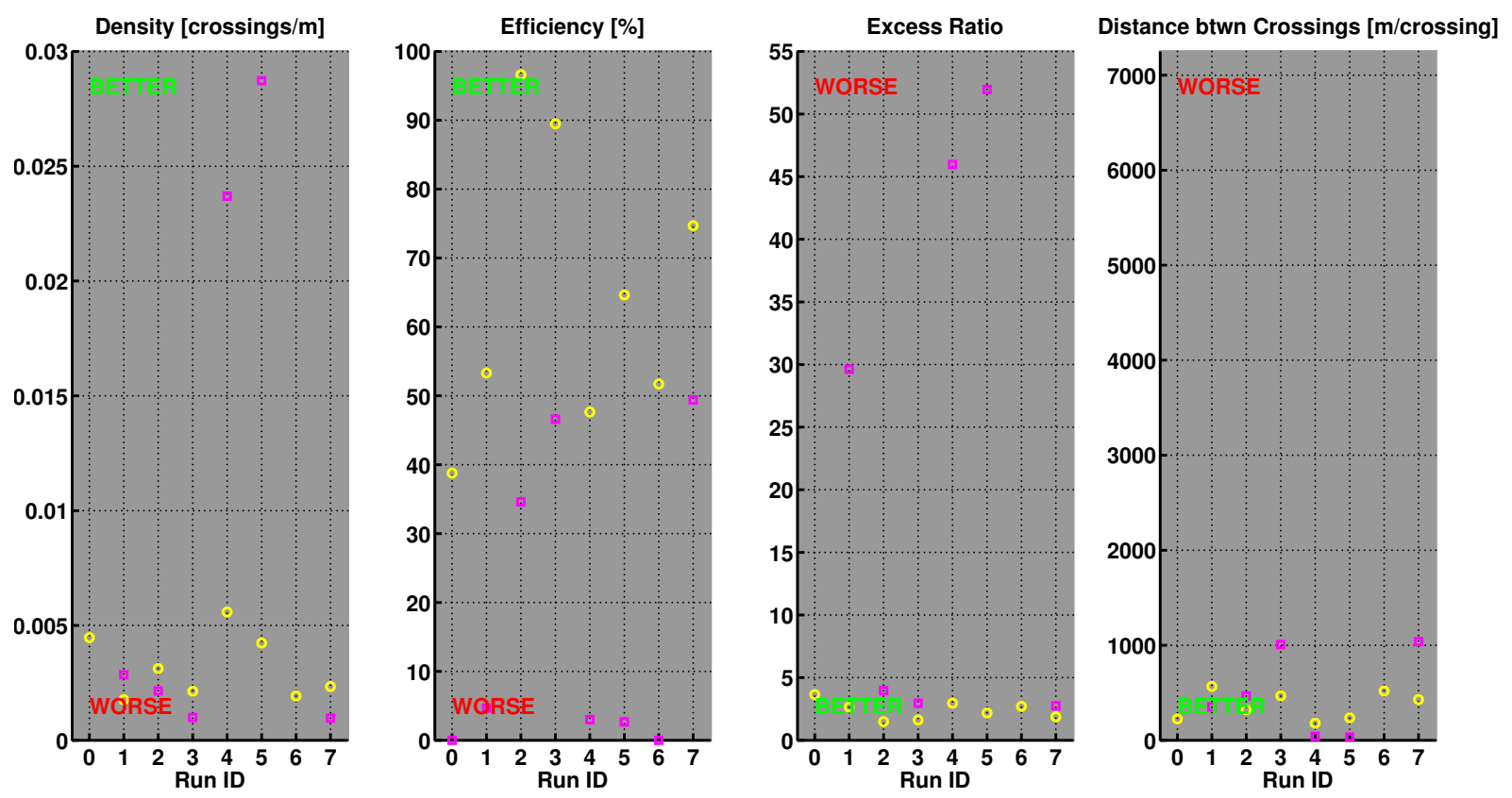

Figure 5-22: Runs 0-7: Performance metrics for Macrura's preplanned zigzag missions (pink squares) and Unicorn's adaptive front tracking missions (yellow circles), plotted for each virtual experiment run. Runs 0-7 evaluated effect of zigzag amplitude on the preplanned $\&$ adaptive missions. The zigzag amplitudes evaluated were $100 \mathrm{~m}$ (Runs 0 \& 4), $500 \mathrm{~m}$ (Runs 1 \& 5), $1000 \mathrm{~m}$ (Runs $2 \&$ 6), and $5000 \mathrm{~m}$ (Runs 3 \& 7). Each zigzag amplitude was evaluated at two model time ranges. Better and worse values for each performance metric are indicated on the plots.

decrease in adaptive zigzag performance as the zigzag amplitude increases. 
Runs 8-11: 2D, both adaptive, collaborative AUV following, follow-the-leader vs. rubber band behaviors

These 2D, 2-AUV (both adaptive) virtual experiments maintained constant AUV zigzag amplitude and operational depth while varying the AUV' collaborative following behavior, aiming to collect data for comparison of the front tracking performance while using BHV_FollowTheLeader vs. BHV_RubberBand to control the AUVs' relative positioning to each other while tracking the front.

Virtual experiment Runs 8-11 evaluated both the new follow-the-leader (acting on both AUVs) and old rubber band (acting on just the the 'following' AUV, Macrura) behaviors for use with 2D multi-AUV front tracking. In each run, both AUVs (Unicorn \& Macrura) were adaptively tracking the front, and Macrura was following Unicorn using either BHV_FollowTheLeader (Runs 8 \& 10) or BHV_RubberBand (Runs 9 \& 11) at two time ranges in the MSEAS model. The resulting performance metrics for both AUVs are plotted in Fig. 5-23. If Macrura is doing a good job following Unicorn and tracking the same front, it would be expected to see both AUVs with relatively close performance metrics values, as is observed for Density, Efficiency, and Distance between Crossings. The only measure with significant differences in value are the Excess Ratios, in which it is seen that, over both time ranges, the rubber band behavior (which is only acting on Macrura and does not affect Unicorn's motion) tends to improve Macrura's Sampling Efficiency. However, with the follow-the-leader behavior an overall improvement is observed in Sampling Efficiency for both AUVs that is about on par with or greater than the Efficiency of Macrura using the rubber band behavior.

In summary, the results and analysis presented above from the preformance comparison of the followthe-leader and rubber band 2D multi-AUV adaptive front tracking behaviors suggest that, based on the few data points available, BHV_Follow TheLeader is an improvement over BHV_RubberBand for 2D multi-AUV adaptive front tracking. 

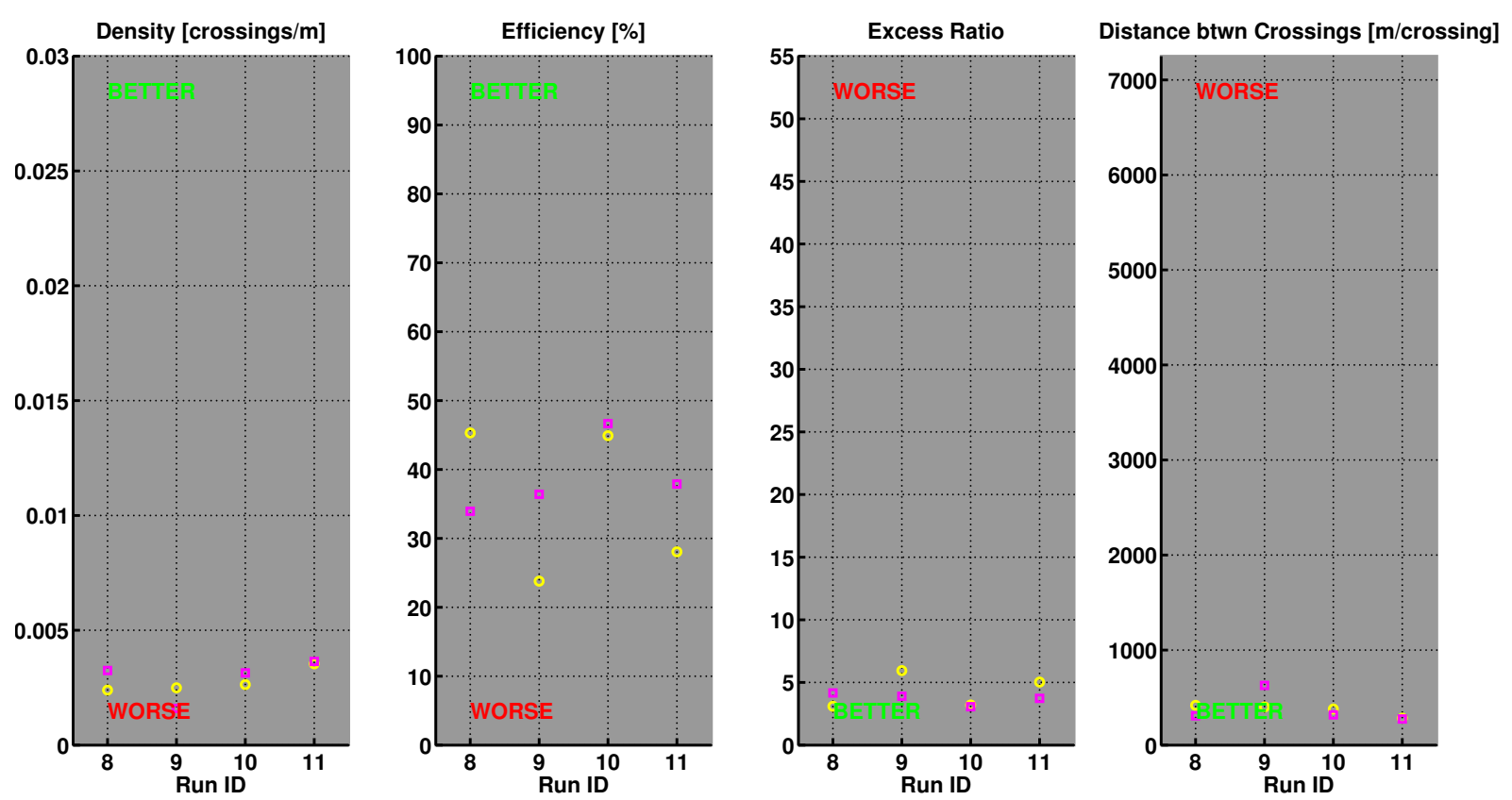

Figure 5-23: Runs 8-11: Performance metrics for 2D multi-AUV adaptive front tracking missions, plotted for each virtual experiment run. Macrura (pink squares) was following Unicorn (yellow circles) using either BHV_FollowTheLeader (Runs 8 \& 10) or BHV_RubberBand (Runs $9 \& 11$ ) at two time ranges in the MSEAS model. Each multi-AUV behavior was evaluated at two model time ranges. Better and worse values for each performance metric are indicated on the plots.

\section{Runs 12-13: 2D, 3-AUV comparison, adaptive vs. preplanned straight vs. preplanned front-following zigzag}

These 2D, 3-AUV (1 adaptive, 1 preplanned straight, 1 preplanned front-following) virtual experiments maintained constant AUV zigzag amplitudes and operational depth, aiming to collect data for a comparison of adaptive zigzag, preplanned straight zigzag, and preplanned front-following zigzag front tracking methods.

Runs $12 \& 13$ experiment with comparing 3 AUVs, each with a different method of sampling the front. The usual two AUVs, Unicorn and Macrura, perform their basic adaptive and preplanned straight zigzag missions along the front, respectively, while a third AUV (Neptune) follows a set of hand-selected waypoints in a preplanned front-following zigzag pattern. Neptune's waypoints were selected to achieve full across-front coverage while roughly crossing the front at the same front intersect angle $\left(45^{\circ}\right)$ as Unicorn and Macrura's missions. The waypoints are given in Table 5.10 and plotted over the operational area in Fig. 5-24. These front-following zigzag points were selected from a horizontal slice of temperature from the MSEAS model at the AUVs' operational depth $(30 \mathrm{~m})$ and a random time within the model's bounds to best approximate the data an AUV operator might have to base a preplanned front-following mission off of. The only difference in 
this case versus an actual deployment is that the temperature map used to plan the waypoints along the front is at the AUV operational depth, whereas an operator on an actual deployment is most likely to have only a recent satellite sea surface temperature (SST) image, not a map at depth. This suggests that the data from the preplanned front-following zigzag in these virtual experiments would likely show better performance results than one that could be planned for a real deployment using SST maps.

Table 5.10: Neptune's preplanned frontfollowing waypoint pattern in the local X-Y grid relative to the Datum $\left(39.409291^{\circ} \mathrm{N}\right.$, $\left.71.934359^{\circ} \mathrm{E}\right)$.

\begin{tabular}{|l|l|l|}
\hline Waypoint ID & $\mathbf{X}[\mathbf{m}]$ & $\mathbf{Y}[\mathbf{m}]$ \\
\hline \hline Start & 100000 & 113000 \\
\hline 1 & 100000 & 105000 \\
\hline 2 & 114000 & 111000 \\
\hline 3 & 123000 & 97000 \\
\hline 4 & 123000 & 112000 \\
\hline 5 & 131000 & 97000 \\
\hline 6 & 145000 & 113000 \\
\hline 7 & 145000 & 93000 \\
\hline 8 & 162000 & 103000 \\
\hline 9 & 172000 & 85000 \\
\hline 10 & 180000 & 104000 \\
\hline 11 & 196000 & 80000 \\
\hline
\end{tabular}

Results from these two virtual experiment runs are plotted in Fig. 5-25 and performance metrics values are given in Table 5.11 for the adaptive vs. straight zigzag and the adaptive vs. front-following zigzag.

Table 5.11: Percentage of Runs 12-13 where adaptive missions were improvements over the preplanned zigzag missions, according to the performance metrics.

\begin{tabular}{|l|l|l|l|l|}
\hline \multirow{2}{*}{ Run IDs } & \multicolumn{2}{|l|}{$\begin{array}{l}\mathbf{1 2 - 1 3} \text { (vs. } \\
\text { straight zigzag) }\end{array}$} & \multicolumn{2}{l|}{$\begin{array}{l}\text { l2-13 (vs. } \\
\text { front-following } \\
\text { zigzag) }\end{array}$} \\
\cline { 2 - 5 } & runs & percent & runs & percent \\
\hline \hline$\rho$ & $2 / 2$ & $100 \%$ & $2 / 2$ & $100 \%$ \\
\hline$\epsilon$ & $0 / 2$ & $0 \%$ & $0 / 2$ & $0 \%$ \\
\hline$E R$ & $0 / 2$ & $0 \%$ & $0 / 2$ & $0 \%$ \\
\hline$D_{\text {cross }}$ & $2 / 2$ & $100 \%$ & $2 / 2$ & $100 \%$ \\
\hline
\end{tabular}




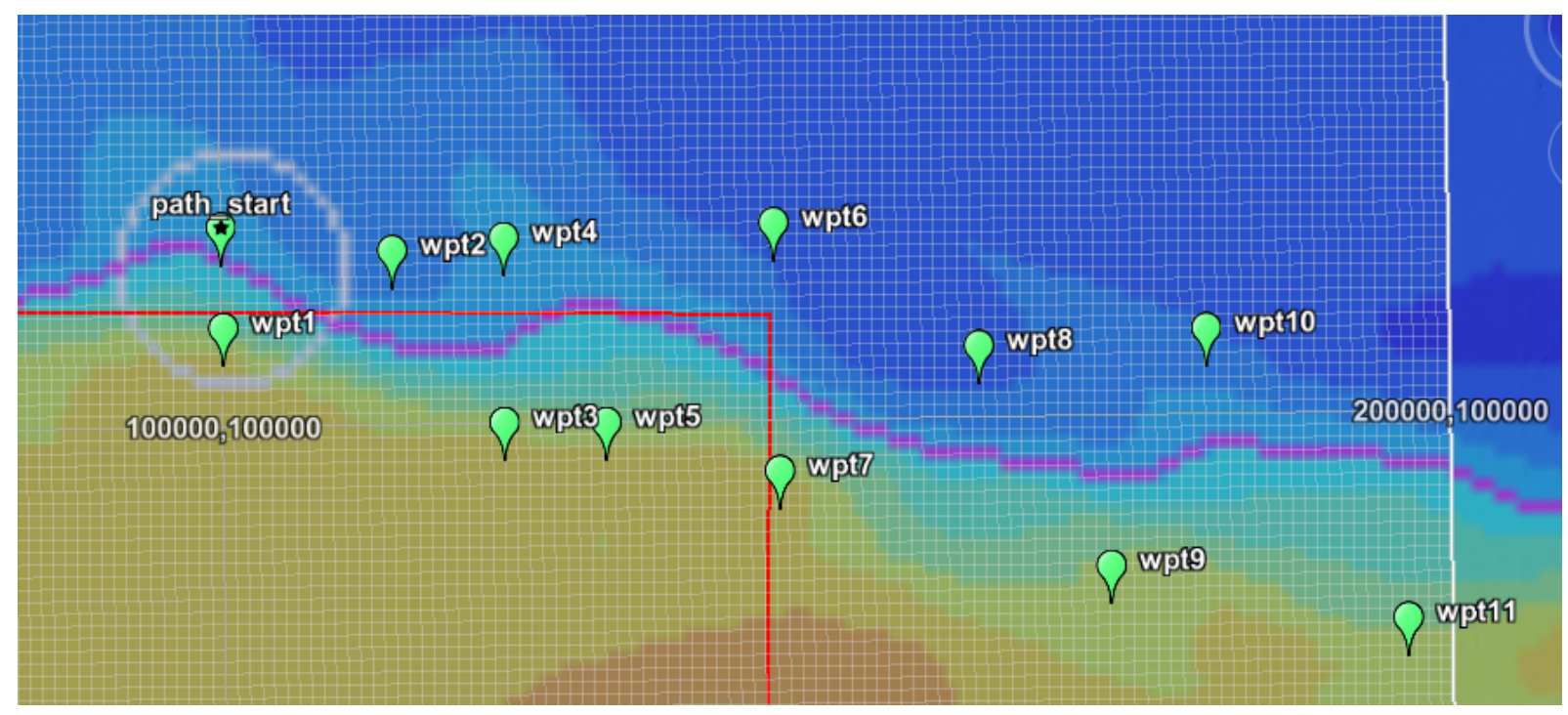

Figure 5-24: The hand-selected points for the preplanned AUV front-following mission along a frontal isotherm at constant depth. The waypoints were selected based on a 'recent' snapshot of the temperature field at the selected AUV operation depth $(30 \mathrm{~m})$ on August 29th, 2006, at 06:00:00 GMT. The frontal isotherm highlighted here with the blurry purple line is at $14.9^{\circ} \mathrm{C}$. The uneven spacing of the waypoints attempts to maintain a local front intersect angle of approximately $45^{\circ}$. The small grid squares are $1 \mathrm{~km} \times 1$ $\mathrm{km}$.

The two missions to compare here to assess the performance of the preplanned (non-adaptive) frontfollowing zigzag are the two preplanned zigzags (Fig. 5-25). The preplanned front-following zigzag exhibited reduced performance when compared to the preplanned straight zigzag for all applicable performance metrics (crossing Density, Efficiency, Excess Ratio, and Distance between Crossings). This is largely due to the selection of the preplanned front-following waypoints, which were more spread out than the straight zigzag waypoints in order to maintain an approximately $45^{\circ}$ front intersect angle while guaranteeing coverage across and along the predicted front (based on a recent horizontal slice of temperature at depth). If instead a smaller-amplitude fixed front-following zigzag pattern had been chosen, it is likely that similar results would have been observed, where the zigzag amplitude is too small to maintain coverage across the front when the front is spatiotemporally dynamic in the small-scale to mesoscale.

When comparing the preplanned front-following zigzag to the adaptive zigzag (Fig. 5-25 and Table 5.11), it is important to note that the adaptive zigzag displayed poor front tracking during both runs (similar to Fig. 5-13). Despite reduced performance of the poorly-executed adaptive zigzag mission compared to that of both preplanned zigzag missions when looking at Efficiency and Excess ratio numbers, the adaptive mission still succeeded in increasing crossing Density and decreasing Distance between Crossings. The caveat here is that the adaptive zigzag did not capture much data in the along-front direction further than about $10 \mathrm{~km}$ from the 

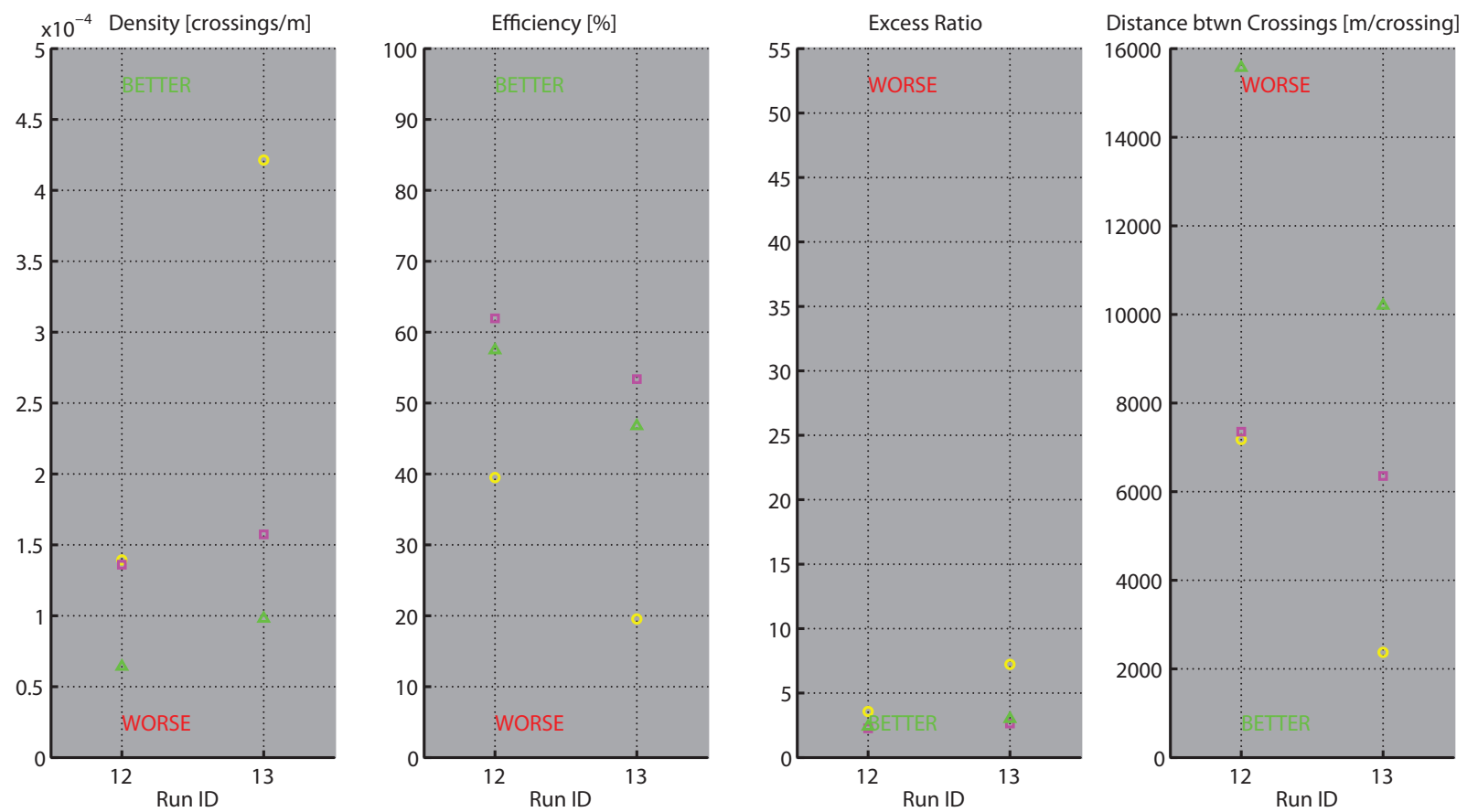

Figure 5-25: Runs 12-13: Performance metrics for Neptune's preplanned front-following zigzag missions (green triangles), Macrura's preplanned straight zigzag missions (pink squares), and Unicorn's adaptive front tracking missions (yellow circles), plotted for each virtual experiment run. These 3-AUV mission sets were evaluated at two model time ranges. Better and worse values for each performance metric are indicated on the plots.

starting location.

In summary, the results and analysis presented above from the comparison of adaptive zigzag, preplanned straight zigzag, and preplanned front-following zigzag methods suggest that the use of this particular preplanned front-following zigzag results in a reduction in performance when compared to the preplanned straight zigzag. It cannot be concluded, however, whether the adaptive zigzag mission was an improvement (or not) over the preplanned zigzag missions without more virtual experiment runs over different time frames, as done in the 2-AUV adaptive vs. preplanned straight zigzag runs (Runs 36-64).

\section{Runs 14-21: 2D, vary detection loiter radius (adaptive)}

These 2D, 2-AUV (1 adaptive, 1 preplanned) virtual experiments maintained constant AUV zigzag amplitudes and operational depth while varying the detection-phase loiter radius of the adaptive AUV, aiming to collect data for an evaluation of the effect of loiter radius on the performance of the adaptive missions.

Runs 14-21 evaluated the effect of initial (detection-phase) loiter radius on the adaptive front tracking zigzag mission. The loiter radii evaluated were 1000 m (Runs 14 \& 18), 2000 m (Runs 15 \& 19), 4000 m 
(Runs $16 \& 20$ ), and $8000 \mathrm{~m}$ (Runs $17 \& 21$ ). Each loiter radius was evaluated at two model time ranges.

The resulting performance metrics are plotted in Fig. 5-26, and general results are given in Table 5.12. The results that matter most in this case, however, are trends of improvement or degradation in front detection and tracking as the loiter radius is changed. There is a general decrease in crossing Density and increase in Distance between Crossings as loiter radius is increased, which suggests that a smaller loiter radius is better. However, the Efficiency plot shows a distinct peak and the Excess Ratio plot shows a marginal minimum with a $4000 \mathrm{~m}$ loiter radius for both time ranges (Runs $16 \& 20$ ). The combination of these results suggests that $4000 \mathrm{~m}$ or slightly smaller may be the optimal loiter radius. The smaller $2000 \mathrm{~m}$ loiter radius was selected for the base adaptive front tracking mission in order to expedite initial detection of the front, though these results suggest that Sampling Efficiency may have been sacrificed in doing so, and the AUV may be traveling slightly more distance than necessary.
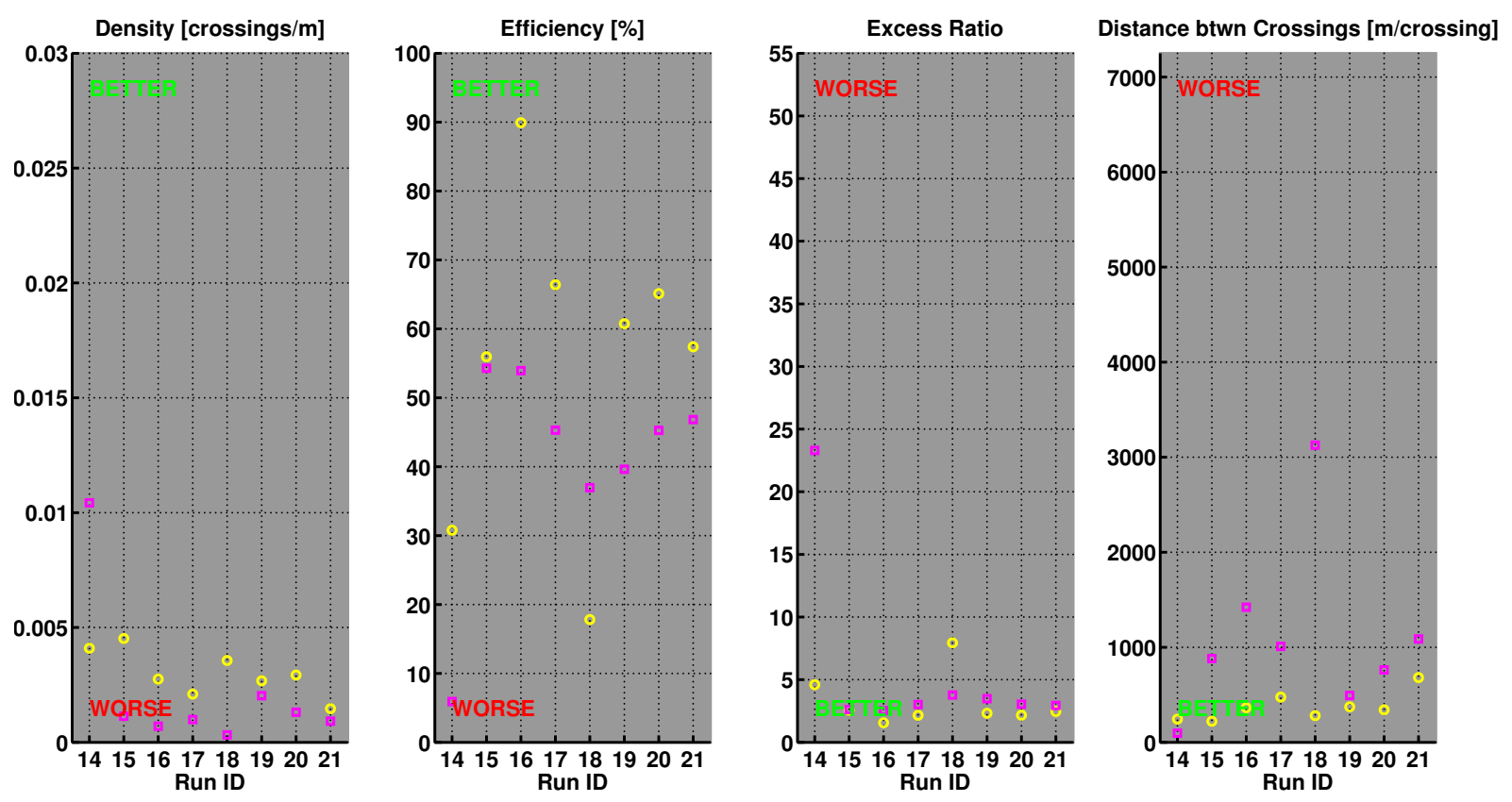

Figure 5-26: Runs 14-21: Performance metrics for Macrura's preplanned zigzag missions (pink squares) and Unicorn's adaptive front tracking missions (yellow circles), plotted for each virtual experiment run. Runs 14-21 evaluated effect of initial loiter radius on the adaptive missions. The loiter radii evaluated were 1000 m (Runs 14 \& 18), 2000 m (Runs 15 \& 19), 4000 m (Runs 16 \& 20), and 8000 m (Runs 17 \& 21). Each loiter radius was evaluated at two model time ranges. Better and worse values for each performance metric are indicated on the plots.

In summary, the results and analysis presented above from the evaluation of the effect of loiter radius on the performance of the adaptive missions suggest that the use of a detection-phase loiter radius of $4000 \mathrm{~m}$ or slightly smaller may be optimal for 2D adaptive front tracking performance. 
Table 5.12: Percentage of Runs 14-21 where adaptive missions were improvements over the preplanned zigzag mission, according to the performance metrics.

\begin{tabular}{|l|l|l|}
\hline \multirow{2}{*}{ Run IDs } & \multicolumn{2}{|l|}{$\mathbf{1 4 - 2 1}$} \\
\cline { 2 - 3 } & runs & percent \\
\hline \hline$\rho$ & $7 / 8$ & $87.5 \%$ \\
\hline$\epsilon$ & $7 / 8$ & $87.5 \%$ \\
\hline$E R$ & $7 / 8$ & $87.5 \%$ \\
\hline$D_{\text {cross }}$ & $7 / 8$ & $87.5 \%$ \\
\hline
\end{tabular}

\section{Runs 22-23: 2D, detection-phase loiter vs. spiral}

These 2D, 2-AUV (both adaptive) virtual experiments maintained constant AUV zigzag amplitude and operational depth, with one AUV using the detection-phase loiter and the other using the detection-phase spiral, aiming to collect data for a comparison of the effect of the detection-phase loiter vs. spiral on the performance of the adaptive missions.

Runs $22 \& 23$ compare the use of the detection-phase initial loiter to the detection-phase initial spiral with both AUVs performing independent 2D adaptive front tracking simultaneously. Macrura (pink) used the initial spiral and Unicorn (yellow) used the initial loiter. The resulting performance metrics are plotted in Fig. 5-27, where each run is a different time range in the model. Here it is observed that the only consistent performance metrics between the loiter and spiral detection phases over the two time ranges are Density and Distance between Crossings (where one is just the inverse of the other). In these cases, it is seen that the initial loiter has better overall results. However, given the toss-up for the Efficiency and Excess Ratio metrics and the fact that only two data points where available, results are inconclusive.

In summary, the results and analysis presented above from the comparison of the effect of the detectionphase loiter vs. spiral on the performance of the adaptive missions suggest that, lacking more data, results are inconclusive as to whether the initial loiter or spiral pattern improves $2 \mathrm{D}$ adaptive front tracking more. 

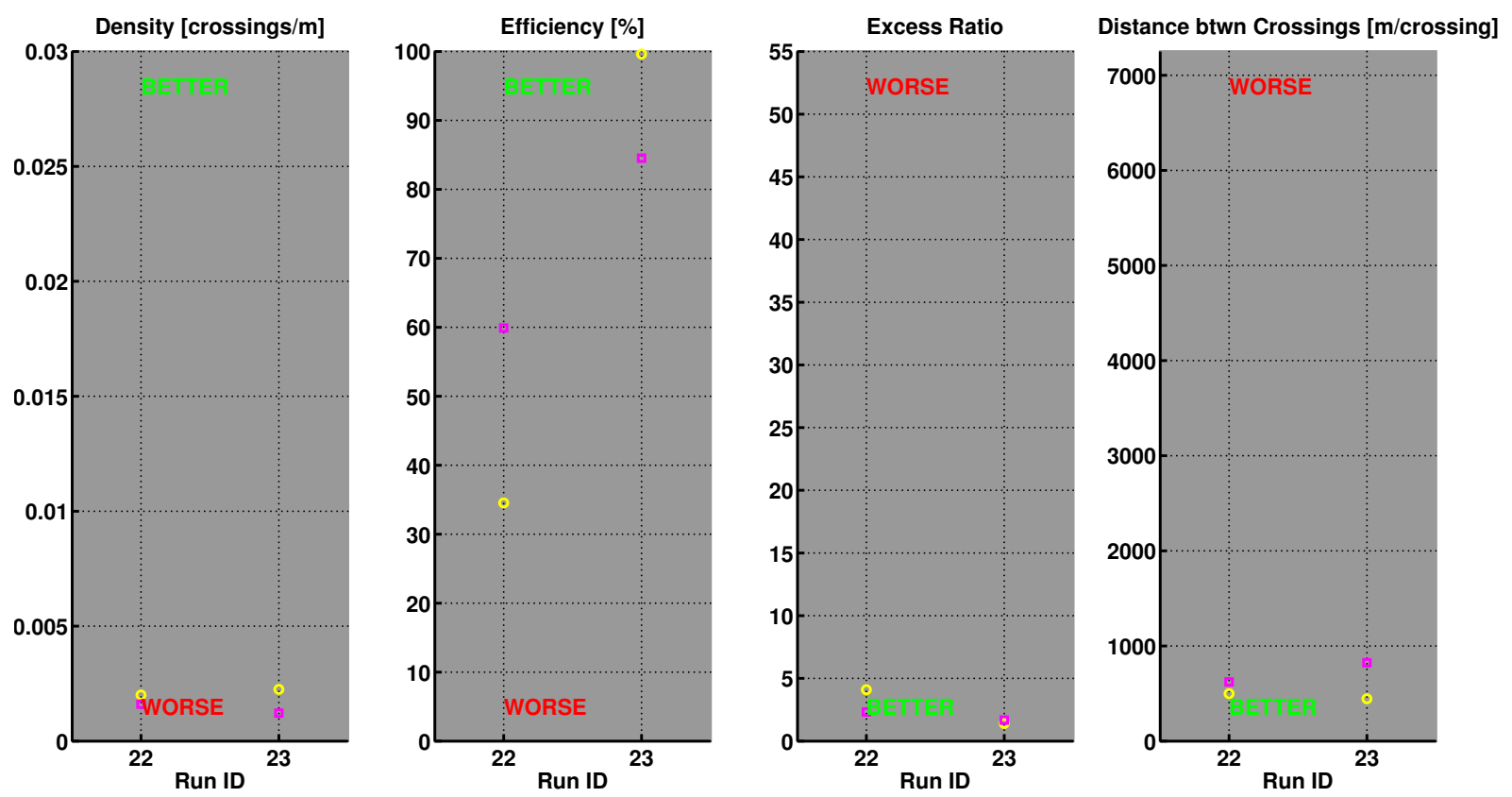

Figure 5-27: Runs 22-23: Performance metrics for 2D adaptive front tracking missions, plotted for each virtual experiment run. Macrura (pink squares) used a detection-phase initial spiral and Unicorn (yellow circles) used a detection-phase initial loiter at two time ranges in the MSEAS model. The two AUVs operated independently to track the front. The loiter vs. spiral missions were evaluated at two model time ranges. Better and worse values for each performance metric are indicated on the plots.

\section{Runs 24-31: 3D, adaptive zigzag vs. adaptive helix}

These 3D, 2-AUV (1 adaptively zigzagging in 2D, 1 adaptively helix-ing in 3D) virtual experiments varied the AUV operational (central) depth and helix dimensions while maintaining constant zigzag amplitude on the adaptively zigzagging $A U V$, aiming to collect data for an evaluation of the performance of the $2 D$ zigzag vs. $3 D$ helix adaptive front tracking behaviors.

Runs 24-31 evaluated the 3D adaptive helix front tracking mission against the $2 \mathrm{D}$ adaptive zigzag front tracking mission. For these runs, Unicorn was assigned to the 2D adaptive zigzag mission and Macrura was running the $3 \mathrm{D}$ adaptive helix mission. These missions were centered at four different depths, and the helix radius was set equivalent to center depth while the spacing between helix loops $(2 \pi c)$ was set to four times the helix radius (to maintain $45^{\circ}$ front intersect angles). The depth-centers selected for the virtual experiments were 30 m (Runs 24 \& 28), 100 m (Runs 25 \& 29), 300 m (Runs 26 \& 30), and 1000 m (Runs 27 \& 31),

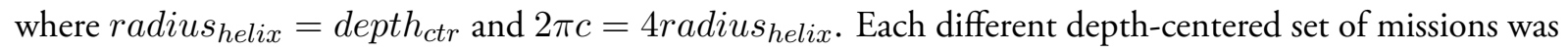
evaluated at two model time ranges. The performance metrics results from these runs are plotted in Fig. 5-28.

For most of these runs, the 3D adaptive helix showed reduced performance (lower Efficiency, higher 

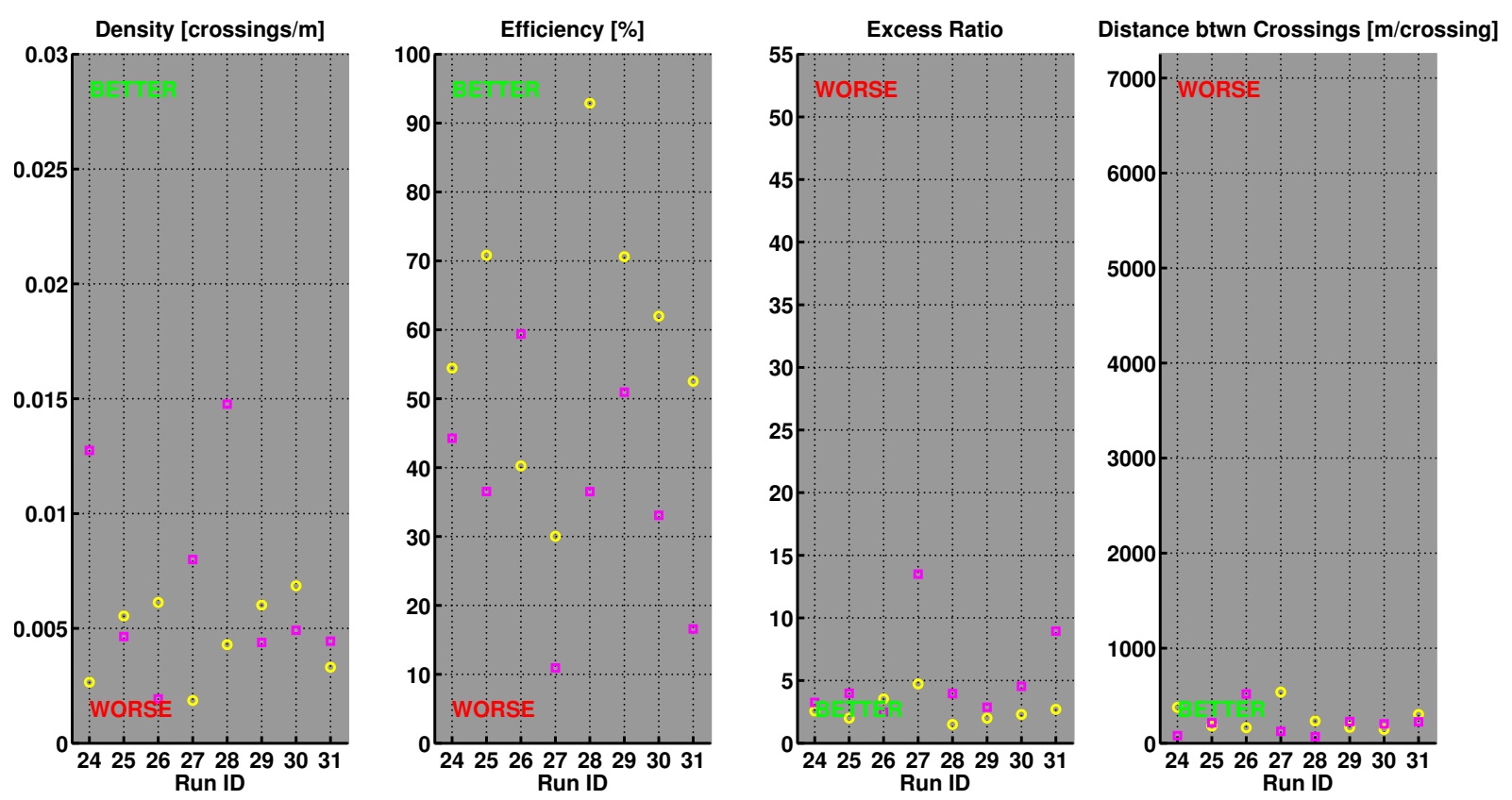

Figure 5-28: Runs 24-31: Performance metrics for Macrura's 3D adaptive helix front tracking missions (pink squares) and Unicorn's 2D adaptive zigzag front tracking missions (yellow circles), plotted for each virtual experiment run. Each different depth-centered set of missions was evaluated at two model time ranges. Better and worse values for each performance metric are indicated on the plots.

Excess Ratio, and —-for half of the runs—reduced crossing Density) when compared to the 2D adaptive zigzag (see Table 5.13), with the lowest Efficiency and greatest Excess while the helix was centered at $1000 \mathrm{~m}$ due to the AUV frequently straying too far from the front over the period of one helix loop. The highest sampling Densities and smallest Distances between Crossings for the helix, however, occurred during the runs centered at $30 \mathrm{~m}$ and $1000 \mathrm{~m}$. For the helix centered at $30 \mathrm{~m}$ this was actually a significant improvement over the 2D zigzags, since the helix tracked the front well with a smaller $(30 \mathrm{~m})$ radius (analogous to a smaller adaptive zigzag amplitude) than the adaptive zigzag with a $500 \mathrm{~m}$ amplitude and thus collected a denser sampling of front data. In the case of the helix centered at $1000 \mathrm{~m}$ having comparatively higher Densities and smaller Distances between Crossings than the 2D adaptive zigzags, this apparent improvement in performance comes with the caveat that the helix was continuously losing the front line and returning to the last detected front crossing location (thus finding a 'new' crossing point right next to the previous one) after re-starting the helix motion, rather than performing the helix behavior to its full extent. This resulted in a glut of front crossings within a small area for the helix centered at $1000 \mathrm{~m}$, but very little tracking along the front using the helix, resulting in very poor performance despite the seemingly good Density and Distance between Crossings metrics. 
Table 5.13: Percentage of Runs 24-31 where 2D adaptive zigzag missions were improvements over $3 \mathrm{D}$ adaptive helix missions, according to the performance metrics.

\begin{tabular}{|l|l|l|}
\hline \multirow{2}{*}{ Run IDs } & \multicolumn{2}{|l|}{$\mathbf{2 4 - 3 1}$} \\
\cline { 2 - 3 } & runs & percent \\
\hline \hline$\rho$ & $4 / 8$ & $50 \%$ \\
\hline$\epsilon$ & $7 / 8$ & $87.5 \%$ \\
\hline$E R$ & $7 / 8$ & $87.5 \%$ \\
\hline$D_{\text {cross }}$ & $4 / 8$ & $50 \%$ \\
\hline
\end{tabular}

This overall reduction in performance from the $2 \mathrm{D}$ to the $3 \mathrm{D}$ adaptive front tracking is expected, given that the 3D helix requires the AUV to travel a longer path (changing depth) as it crosses the front, rather than gathering many points at the fixed helix-center depth. Since fewer points are available to the 3D adaptive helix behavior to determine the presence and location of a front crossing point at the helix-center depth (there may be as few as two points at the center depth on a given rotation of the AUV around the helix) than there are for the $2 \mathrm{D}$ adaptive zigzag behavior at constant depth, there is higher risk of the helix losing the front location as it travels through depth. For the runs centered at $30 \mathrm{~m}, 100 \mathrm{~m}$, and $300 \mathrm{~m}$, in fact, the reduction in performance is somewhat balanced by the data set being collected, which is 3-dimensional in space rather than just 2-dimensional. The 3D data set collected by the helix captures the distribution of temperature with depth, making it possible to approximate the front's structure as a plane in $3 \mathrm{D}$ space rather than just as a $2 \mathrm{D}$ line. This means it is possible to successfully collect a 3D spatial distribution of temperature along the front using this horizontal helix front tracking behavior without the need for a second AUV, with the concession of a slight reduction in performance compared to the $2 \mathrm{D}$ adaptive zigzag behavior.

In summary, the results and analysis presented above from the evaluation of the performance of the $2 \mathrm{D}$ zigzag vs. 3D helix adaptive front tracking behaviors suggest that, despite the slight reduction in performance when compared to the $2 \mathrm{D}$ adaptive zigzag behavior, the use of the $3 \mathrm{D}$ adaptive helix behavior for front tracking is still useful and important for capturing the coupled vertical and horizontal temperature distributions at the frontal interface between two water masses with only one AUV.

\section{Runs 32-33: 2D, adaptive zigzag in $3 \mathrm{~km}$ vs. $1 \mathrm{~km}$ resolution environment, AW06 domain}

These 2D, 2-AUV (both adaptive) virtual experiments maintained constant AUV zigzag amplitude, operational depth and operational area (AW06 domain), with one AUV running in the linearly-interpolated $3 \mathrm{~km}$ resolution SW06 ocean model and the other in the linearly-interpolated $1 \mathrm{~km}$ resolution AW06 ocean model, aiming to collect 
data for a comparison of model resolution (larger-vs. smaller-scale data variation) effects on $2 D$ adaptive front tracking performance.

Runs 32-33 evaluated the performance of 2D adaptive zigzag front tracking in higher-resolution models. For these virtual experiments, both Unicorn and Marcura ran the same 2D adaptive missions individually. The difference between the two AUVs was that Unicorn was running the $1 \mathrm{~km}$ resolution AW06 model and Macrura was running the $3 \mathrm{~km}$ resolution SW06 model (used for all other previously-described runs). The performance in the $1 \mathrm{~km}$ and $3 \mathrm{~km}$ domains was measured using the $1 \mathrm{~km}$ and $3 \mathrm{~km}$ models, respectively. Again, the virtual experiments were evaluated at two model time ranges. The performance metrics results from these runs are plotted in Fig. 5-29 and summarized in Table 5.14.
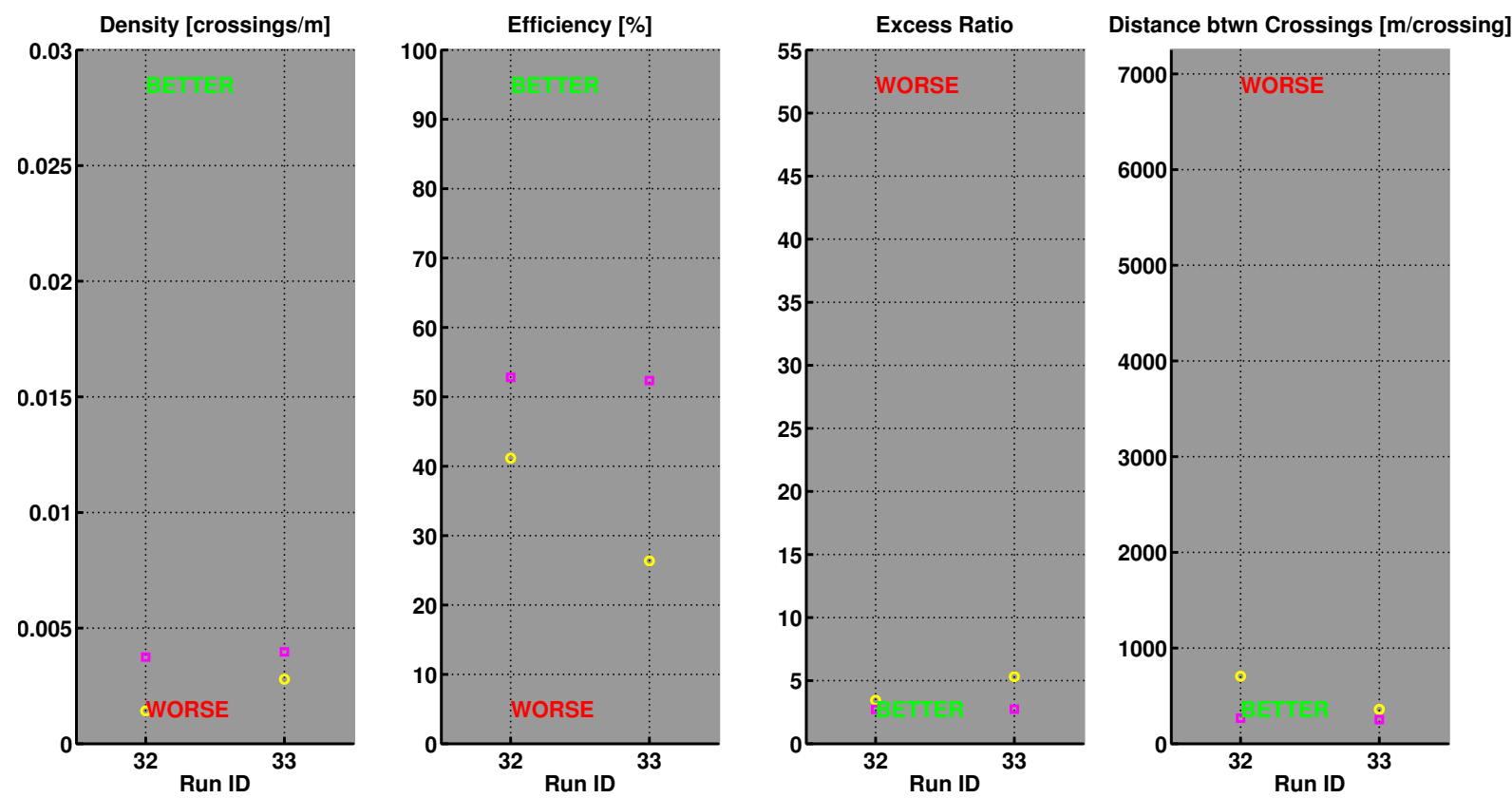

Figure 5-29: Runs 32-33: Performance metrics for 2D adaptive zigzag front tracking missions, where Macrura was running the $3 \mathrm{~km}$ resolution SW06 model (pink squares) and Unicorn was running the $1 \mathrm{~km}$ resolution AW06 model (yellow circles), plotted for each virtual experiment run. The $3 \mathrm{~km}$ vs. $1 \mathrm{~km}$ resolution missions were evaluated at two model time ranges. Better and worse values for each performance metric are indicated on the plots.

Based on the results from Runs 32-33, it is clear that front tracking performance from all performance metrics was reduced when using the higher $(1 \mathrm{~km})$ resolution AW06 model for the virtual experiment environ-

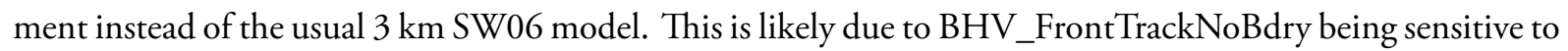
all variations in temperature measured by the simulated CTD sensor sampling the linearly-interpolated gridded model. It is also possible that increased zigzag amplitude or decreased front intersection angle may more 
Table 5.14: Percentage of Runs 32-33 where running a 2D adaptive zigzag missions in a higher-resolution model was an improvement over running the same mission in a lowerresolution model, according to the performance metrics.

\begin{tabular}{|l|l|l|}
\hline \multirow{2}{*}{ Run IDs } & \multicolumn{2}{|l|}{$\mathbf{3 2 - 3 3}$} \\
\cline { 2 - 3 } & runs & percent \\
\hline \hline$\rho$ & $0 / 2$ & $0 \%$ \\
\hline$\epsilon$ & $0 / 2$ & $0 \%$ \\
\hline$E R$ & $0 / 2$ & $0 \%$ \\
\hline$D_{\text {cross }}$ & $0 / 2$ & $0 \%$ \\
\hline
\end{tabular}

robustly sample and track a higher-resolution front by forcing the AUV to move beyond the smaller-scale frontal isotherm variations before changing heading to cross the frontal isotherm again.

If the model resolution is higher, there is less smoothing in the interpolated data, and thus more smallerscale variation that exhibits more complex thermal structure. Actual in-water temperature measurements can have variations on scales that are smaller than $1 \mathrm{~m}$, which is much finer resolution than even the $1 \mathrm{~km}$ resolution AW06 model tested here. The small thermal structure is good to sample and retain in a data set, but can throw off temperature-based autonomy algorithms and behaviors as the temperatures quickly jump around. To reduce the negative effect of in-water small temperature variations on the $2 \mathrm{D}$ mesoscale front tracking behavior presented here, it will be crucial to either employ real-time smoothing or filtering of the collected temperature data, or to tune the zigzag amplitude and front intersection angle to move the AUV well beyond the complex frontal zone on each zigzag leg. (The full-resolution data would still be recorded for future use, before any smoothing could be applied.)

Similar methods for increasing robust handling of noisy temperature data were applied to AUV adaptive thermocline tracking methods by Wang et al. [40] during the FAF05 in-water exercise and prior virtual experiments. Their temperature data were "boxed-averaged" over vertical and horizontal grids with resolutions selected according to the oceanographic scales of interest, and the CTD noise was somewhat reduced by estimating and rejecting the noise probabilistically. They also evaluated a number of behavior configuration values and combinations to tune their system for optimal thermocline tracking, according to the mission objectives. A temperature binning method was also used in the adaptive thermocline tracking work presented in Chapter 3 of this thesis and in Petillo et al. [3]. In the future, similar methods to those mentioned here should be applied to the adaptive front tracking behaviors and temperature data processing described in this chapter to improve performance in more realistic environments. 
Our 2D adaptive front tracking behavior had previously been tested and developed with only the $3 \mathrm{~km}$ resolution model. Thus, these results suggest a necessity for smoothing the temperature data to approximately $3 \mathrm{~km}$ resolution as it is collected. This could be done by low-pass filtering the temperature data, keeping a weighted temperature average over some time window as the 'current' temperature for a given AUV positionreducing the higher-resolution temperature variations-, or by binning the temperature data spatially based on a $3 \mathrm{~km}$ grid superimposed on the local $\mathrm{x}-\mathrm{y}$ coordinate grid. The former two ideas have potential to be simple yet robust solutions. The latter would require essentially constructing, storing, and constantly updating a basic gridded model of collected temperature data and performing complex interpolation as is done with the MSEAS models in virtual experiments and in [40], which can be processor-intensive. Another detractor from using any sort of binning or low-pass filtering to a $3 \mathrm{~km}$ resolution would be the subsequent inability of the AUV to accurately track more closely to the front to collect data from smaller-scale features such as any turbulence structure at the frontal interface. Alternately, or in addition, further tuning of the adaptive zigzag amplitude and front intersection angle can be evaluated and applied through future virtual experiments to improve the adaptive front tracking performance in the $1 \mathrm{~km}$ and more realistic higher-resolution models.

In summary, the results and analysis presented above from the comparison of $3 \mathrm{~km}$ and $1 \mathrm{~km}$ model resolution effects on $2 \mathrm{D}$ adaptive front tracking performance suggest that, for all performance metrics, the use of the higher-resolution $(1 \mathrm{~km}$ ) model reduced performance of the adaptive zigzag behavior (when using the tuned configuration parameters for virtual experiments in the $3 \mathrm{~km}$ resolution model) due to the behavior's sensitivity to all variations in temperature. Thus, it will be necessary to improve this behavior by low-pass filtering or binning the environmental data to approximately $3 \mathrm{~km}$ resolution for use in both higher-resolution models and in-water deployments. Additionally, further tuning based on performance evaluation of adaptive zigzag amplitude and front intersection angle should be completed and applied for improving adaptive front tracking in the $1 \mathrm{~km}$ and higher-resolution models.

\subsection{Receiver Operating Characteristic Curves}

Receiver operating characteristic (ROC) curves may also be applied to the virtual experiment data as a measure of front tracking performance. These curves compare the probability that the AUV performing adaptive 2D front tracking will correctly detect a front (probability of detection, $P_{D}$ ) to the probability that it will falsely detect a front (probability of false alarm, $P_{F A}$ ), for a variety of threshold values. The ROC curve for front tracking is generated based on the probability density functions (PDFs) of the magnitudes of the temperature gradients $(|\partial T / \partial r|)$ in horizontal space at the front while front tracking (signal plus noise) and away from 
the front (noise). From these PDFs, the $P_{D}$ and $P_{F A}$ can be determined as the area under the curves for the on-front and away-from-front AUVs, respectively, to the right of a threshold temperature gradient value. The threshold value is selected based on the temperature change between the ends of each adaptive zigzag leg and on the zigzag amplitude. Fig. 5-30 plots the resulting ROC curve for the $2 \mathrm{D}$ isotherm-based adaptive zigzag front tracking method presented in this work against the ROC curve for thermocline tracking that was presented in Chapter 3. This suggests that this front tracking method can have a decent probability of detection (80\%) with a reasonably small probability of false alarm (17\%).

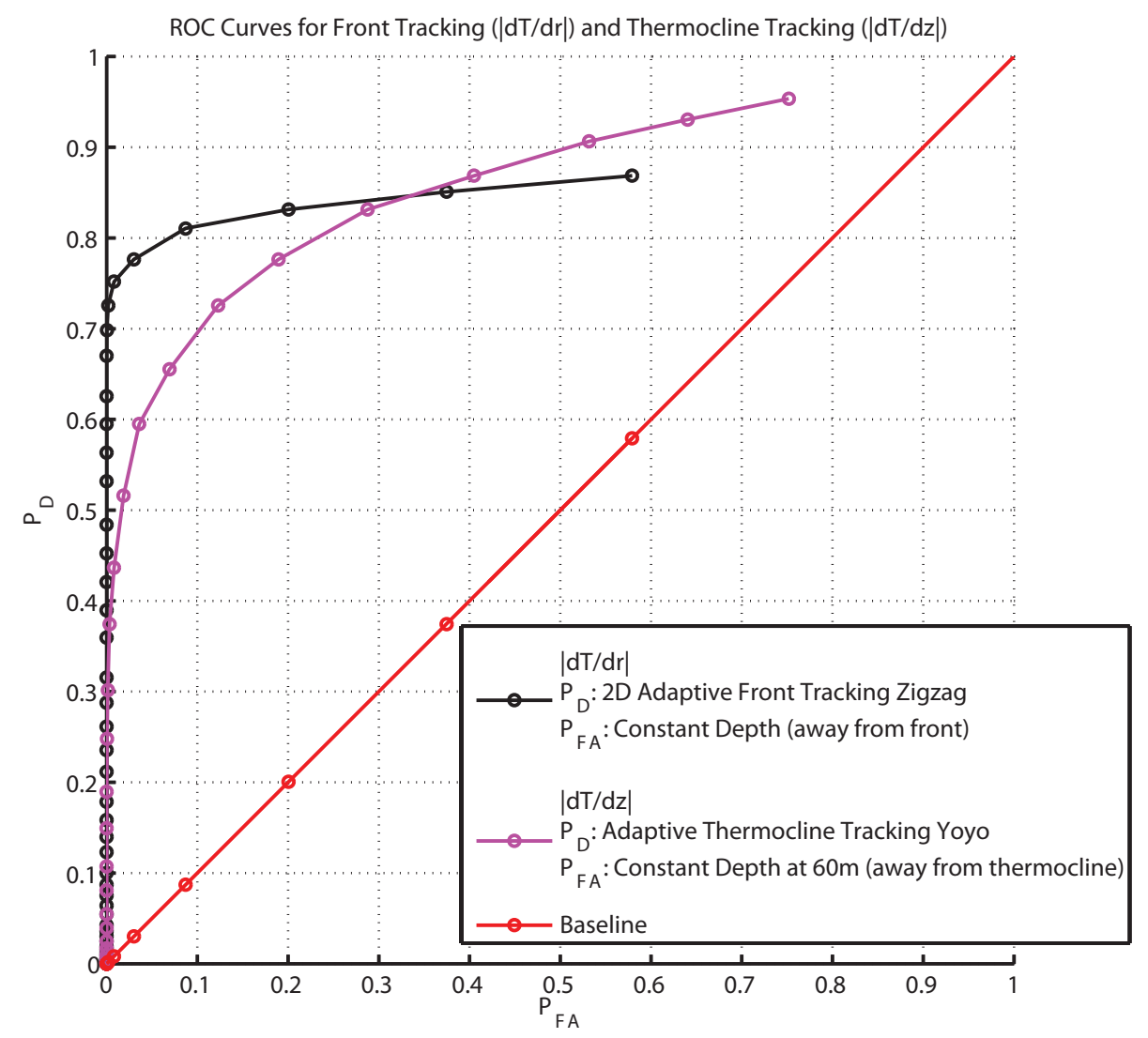

Figure 5-30: ROC curves comparing the performance of the 2D adaptive front tracking method $(|\partial T / \partial r|$, black) to the adaptive thermocline tracking method from Chapters 3 and 4 ( $|\partial T / \partial z|$, magenta). The red curve is a baseline for comparison, where the probability of detection, $P_{D}$, is equal to the probability of false alarm, $P_{F A}$. Curves following the left and top edges of the plot exhibit 'better' performance.

\subsection{Conclusion}

The goals of the work presented in this chapter were to apply AAEA and Feature Tracking to adaptively sample along and across an ocean front using only the data collected on board AUVs, gathering a synoptic data set of 
the position of the front over time while improving sampling efficiency and density over current preplanned AUV sampling surveys.

To this end, three adaptive autonomy behaviors were developed for front tracking in 2D and 3D space, with single and multiple AUVs: BHV_FrontTrackNoBdry (2D front-following zigzag), BHV_FrontTrackHelix (3D front-following helix), and BHV_FollowTheLeader (multi-AUV coordination for front tracking in the horizontal plane). A number of performance metrics were developed for comparative evaluation of these behaviors. The $2 \mathrm{D}$ front tracking behavior's performance was also evaluated against that of a preplanned zigzag survey (representative of current methods used for collecting data along a front). A spatiotemporally dynamic MSEAS model of the Mid-Atlantic Bight (MAB) region off the east coast of the U.S. was used as a testing environment for virtual experiments, allowing these new AUV front tracking methods to be evaluated.

The front tracking behaviors presented here are essentially isotherm-following behaviors, since the acrossfront temperature gradient can vary in the along-front direction and the uncertain angle at which an AUV crosses the front and samples the temperature would affect the apparent temperature gradient at a given location. The front tracking behaviors use a three-phase process (conceptualized in Fig. 5-5) to achieve front isotherm tracking:

1. Detection: The AUV performs a survey of the local region to detect the front (loiter or spiral behavior), an isotherm is selected to represent the front line, and a minimum of three front 'crossing' points are collected to trigger the classification phase.

2. Classification: The AUV estimates the local front as a line using a weighted linear least squares approximation, requiring at least three crossing points to be within a specific spatiotemporal range of the current time and AUV position to produce the linear approximation for tracking to begin.

3. Tracking: The heading of the AUV is set to intersect the front line estimate at a $45^{\circ}$ angle. The front estimate is updated when the AUV crosses the front on that heading, and the heading required to intersect the front again (also at a $45^{\circ}$ angle) is determined and set once the AUV has traveled a specified distance from the front. This results in a zigzag path along the front that follows the front as it shifts over space and time.

Overall, the 2D adaptive front tracking behavior presented here succeeded in improving front mapping performance over that of a preplanned straight zigzag pattern at least $58 \%$ of the time in virtual experiments (Runs 36-64: adaptive vs. preplanned straight zigzag). The performance metrics used to evaluate the adaptive and preplanned front tracking behaviors are summarized in Table 5.15. Adaptive front tracking had the largest 
Table 5.15: Summary of performance metrics equations.

\begin{tabular}{|c|c|c|c|}
\hline Performance Metric & Equation & $\begin{array}{l}\text { Better } \\
\text { Values }\end{array}$ & Significance \\
\hline $\begin{array}{l}\text { Crossing Density } \\
{[\text { crossings } / \mathbf{m}]}\end{array}$ & $\rho=\frac{N_{\text {cross }}}{D_{\text {on_front }}}(5.21)$ & higher* & $\begin{array}{l}\text { How many front crossings were } \\
\text { made by the AUV per unit } \\
\text { length of the front line that was } \\
\text { tracked }\end{array}$ \\
\hline $\begin{array}{l}\text { Sampling Efficiency } \\
{[\%]}\end{array}$ & $\epsilon=\frac{D_{\text {on_front }}}{D_{\text {front }}} \times 100 \%(5.23)$ & higher & $\begin{array}{l}\text { The percentage of } D_{\text {front }} \text { that } \\
\text { was tracked and sampled by the } \\
\text { AUV }\end{array}$ \\
\hline Excess Ratio & $E R=\frac{D_{\text {total }}}{D_{\text {on_front }}}(5.24)$ & lower* & $\begin{array}{l}\text { How much of the AUV's travel } \\
\text { distance was in excess of the } \\
\text { distance along the front that the } \\
\text { AUV captured the front }\end{array}$ \\
\hline $\begin{array}{l}\text { Distance between } \\
\text { Crossings } \\
\text { [m/crossing] }\end{array}$ & $D_{\text {cross }}=\frac{1}{\rho}(5.22)$ & lower* & $\begin{array}{l}\text { The average distance the AUV } \\
\text { traveled between front crossings }\end{array}$ \\
\hline $\begin{array}{l}\text { Front Estimate } \\
\text { Error }[\mathrm{m}]\end{array}$ & $\begin{array}{l}F E E= \\
D_{\text {from_front }} @|\partial T / \partial r|_{\max } \text { on } \\
\text { a zigzag leg }(5.25)\end{array}$ & lower & $\begin{array}{l}\text { Compares the }|\partial T / \partial r|_{\max } \\
\text { location to the local estimated } \\
\text { front location, as captured by } \\
\text { the AUV }\end{array}$ \\
\hline $\begin{array}{l}\text { Tracking } \\
\text { Confidence [\%] }\end{array}$ & $\begin{array}{l}T C=2\left(\frac{N^{+}}{N_{t o t}}\right) \times 100 \% \\
(5.26)\end{array}$ & higher & $\begin{array}{l}\text { An evaluation of the confidence } \\
\text { level that the actual front was } \\
\text { followed/sampled by the AUV, } \\
\text { expressed as a percentage }\end{array}$ \\
\hline
\end{tabular}

* It is often desirable to maintain some across-front motion of the AUV, thus extremes of huge $\rho$ and tiny $D_{\text {cross }}$ and $E R$ values are not necessarily optimal.

and most consistent impact on front Crossing Density $(\rho)$ and Distance between Crossings $\left(D_{\text {cross }}\right)$, where it was an improvement over the preplanned straight zigzag $89.7 \%$ of the time. Adaptive front tracking showed less stark Sampling Efficiency $(\epsilon)$ and Excess Ratio $(E R)$ improvements over the preplanned straight zigzag, displaying improved numbers $62.1 \%$ and $58.6 \%$ of the time, respectively. It is also apparent that the start location of the front tracking missions affects the performance of adaptive versus preplanned front tracking. When the front tracking missions were moved north onto the shelf from the old mission area south of the shelfbreak, the adaptive front tracking algorithms tended to track the front worse than the preplanned zigzag despite the stronger across-front temperature gradient in the more northerly location. This was due to a mesoscale slope-water eddy surrounded by an isotherm of the same temperature as the front that Unicorn (adaptive AUV) was stuck in while trying to track along the front north of the MAB shelfbreak. The average Tracking Confidence (TC) for the adaptive front tracking missions in Runs 36-64 was calculated as 59.2\%, 
with a standard deviation of $9.5 \%$, suggesting that the actual shelfbreak front is usually sampled in $25 \%-34 \%$ of the range bins around the estimated front line. For the majority of the runs, the adaptive mission's Front Estimate Error $(F E E)$ value was under $400 \mathrm{~m}$, with standard deviations mostly under $150 \mathrm{~m}$, suggesting that the adaptive AUV tracked fairly close to the 'actual' front line. It is important to note here that the TC and FEE values are only valid for $3 \mathrm{~km}$ resolution environments with linear interpolation (or similar) between grid points and are not valid for higher-resolution models or the actual ocean environment without further improvement to the front tracking behavior. In addition, the passing of tropical storm Ernesto in the MSEAS model had little discernible effect on the adaptive front tracking performance.

Runs 0-7 (varying zigzag amplitudes) suggested that zigzag amplitudes of $\mathrm{O}(1000 \mathrm{~m})$ or more are best for the preplanned straight zigzag, while there is no definitive improvement to using a larger versus smaller adaptive zigzag in the range of $100-5000 \mathrm{~m}$ amplitudes.

Runs 8-11 (multi-AUV follow-the-leader vs. rubber band) showed that both follow-the-leader and rubber band behaviors were suitable for 2D multi-AUV adaptive front tracking, but that the follow-the-leader behavior tended to improve the front tracking of both AUVs over using the rubber band behavior, whereas the rubber band only improved the 'follower' AUV's front tracking.

Runs 12-13 (adaptive vs. preplanned straight vs. preplanned along-front zigzag) are inconclusive as to the improvement of the adaptive zigzag mission over the preplanned zigzag missions, or vice versa. More runs during different model time ranges are needed, as done in Runs 36-64, to draw more definitive conclusions. In addition, the set of preplanned front-following waypoints selected for these runs results in a reduction in performance when compared to the set of preplanned straight zigzag waypoints used, suggesting that preplanned front-following waypoints that are hand-selected by a person are not necessarily an improvement in the case of a temporally dynamic front.

Runs 14-21 (varying adaptive loiter radius) suggest a significant Sampling Efficiency gain from a detectionphase initial loiter radius of approximately $4000 \mathrm{~m}$, though the range of $2000-4000 \mathrm{~m}$ for loiter radius is acceptable due to the slight gain in crossing Density at lower radii without sacrificing too much Sampling Efficiency.

Runs 22-23 (adaptive loiter vs. spiral) hint at only marginal adaptive front tracking improvement from using the detection-phase initial loiter over the initial spiral. Coupled with the fact that only two data points were available, results are inconclusive as to whether the initial loiter versus spiral pattern actually improves $2 \mathrm{D}$ adaptive front tracking more.

Runs 24-31 (2D adaptive zigzag vs. 3D adaptive helix) suggest a general reduction in front tracking 
performance when using the $3 \mathrm{D}$ adaptive horizontal helix compared to the $2 \mathrm{D}$ adaptive zigzag, except when using the helix centered at $30 \mathrm{~m}$. This reduction in performance is due to-and somewhat balanced by-the fact that the helix is also collecting data over a continuous depth range (rather than just one depth) along and across the front. That is, the $2 \mathrm{D}$ adaptive zigzag behavior collects all of its data points at a constant depth (thus, it has a lot of data at the depth at which it is determining the front), whereas the $3 \mathrm{D}$ adaptive helix behavior may have as few as two points collected at its center depth (at which it is determining the front) per period of travel around the helix, making the helical front tracking much more sensitive to spurious data values or smaller-scale temperature variations than the zigzag front tracking at constant depth. The $3 \mathrm{D}$ motion of the helix will, however, allow the front to be approximated (in future work) as a plane instead of just a line, and it adds a depth dimension to the coverage of the front where helical front tracking occurs.

Runs 32-33 (2D adaptive front tracking in a $3 \mathrm{~km}$ vs. $1 \mathrm{~km}$ resolution environment) show that, due to poor performance with an increase in model resolution, some form of real-time temperature data binning (low-pass filtering) may be necessary to achieve the same 2D adaptive front tracking performance results with higher-resolution models and real data as done with the $3 \mathrm{~km}$ resolution SW06 model. Additionally, further tuning based on performance evaluations of varying adaptive zigzag amplitude and front intersection angle parameters should be completed and applied to improve adaptive front tracking in the $1 \mathrm{~km}$ and higherresolution models (similar to Runs $0-7$ ).

An ROC curve performance analysis of the $2 \mathrm{D}$ adaptive front tracking method presented in this chapter suggests that this front tracking method can have a decent probability of front detection and tracking (80\%) with a reasonably small probability of false alarm (17\%). Although this is not ideal, it is very reasonable given the dynamic environmental conditions and is on par with the ROC curve performance of the thermocline tracking method.

Ultimately, the added complexity of using adaptive front tracking to sample along a front must be weighed against the amount of human-AUV interaction and resources necessary to deploy a preplanned AUV survey for the the same purpose. Preplanned surveys require an initial survey of the area (with ship-deployed or towed instruments, AUVs, satellite data, or a combination thereof) to find clues as to where the front might be, AUV deployment on a preplanned path to collect data, AUV recovery or significant time with the AUV on the surface to upload the data to the operators and scientists on the ship, time for human-based (often computer-aided) data analysis and planning a new mission, and finally AUV redeployment with the new mission. This preplanned survey cycle must continue until a sufficient data set has been collected or available shipboard time has run out. As a counterpoint to preplanned front surveys, adaptive front tracking only 
requires the operator or researcher to have a rough idea of where a front might be, as the AUV will determine the exact location and follow the front itself, requiring no shipboard data processing and no redeployment or planning of new missions to maintain sampling along the front. This significantly reduces the time the AUV spends not tracking the front (i.e., on the surface awaiting redeployment or conducting a much larger amplitude zigzag to ensure frontal coverage) and frees up a ship's resources for other scientific experiments to be performed simultaneously. In conclusion, autonomous and adaptive front tracking techniques add upfront complexity to an AUV's software, but, once implemented, significantly reduce the labor and uncertainty involved in efficiently gathering a synoptic data set characterizing an oceanographic front, making the use of autonomous and adaptive front tracking methods worthwhile. 


\section{Chapter 6}

\section{Plume Tracking}

\subsection{Introduction}

The ability to detect and track underwater plumes in an ever more efficient manner is relevant to both scientists and civilians alike. These underwater plumes may be in the form of hydrothermal vent plumes found deep in the ocean, oil spills which may be far out at sea or near coasts or fishing grounds, harmful algal blooms (HABs) that cause beach closures and make exposed shellfish toxic to humans, river outflow plumes of chemicals or suspended sediment, plumes of tracer dye, etc. Each type of plume has specific physical, chemical, and biological properties, as well as characteristic spatial and temporal scales over which the plume's area of coverage changes significantly. Dynamic ocean features such as these are best sampled from a variety of perspectives (using complimentary sensor measurements and/or taking measurements from different positions inside or outside of the plume), of which a few approaches are described in $[4,39,41,42,46,141-144]$.

The plume detection and tracking techniques described in the aforementioned papers have the common approach of using AUVs to complete the bulk of the plume sampling, employing autonomous plume detection and tracking algorithms on board the AUVs when possible. However, for each group, a different type of AUV is used to detect and track a different type of plume using different autonomy algorithms. These differences make it difficult to compare the individual approaches to plume tracking. Thus, we propose to evaluate a variety of AUVs based on their capabilities (design, mobility, deployment duration, on-board processing

Portions of this chapter are (C2010 IFAC. Reprinted, with permission, from S. Petillo and H. Schmidt, "Autonomous and Adaptive Plume Detection and Tracking with AUVs: Concepts, Methods, and Available Technology," Proceedings of the 9th IFAC Conference on Manoeuvring and Control of Marine Craft. [5] 
power, etc.) in an attempt to find an optimal plume-to-AUV match.

Finally, we discuss the various autonomous and adaptive plume detection and tracking techniques that have been tested and suggest a system of our own involving the use of a fleet of autonomously collaborating AUVs that communicate in near real time using acoustic transmissions and adapt their motion to changes in the environment to best detect and track a plume. Part of this approach is similar to the front tracking methods described in Chapter 5 and, thus, employs the AAEA and Feature Tracking method described in Chapter 3. Here we will also account for the fact that, depending on plume type, the AUV(s) will be either looking for the approximately 2D spatial boundary of the plume's non-buoyant layer in the horizontal plane, or for the plume's source location.

\subsection{Plumes}

Plumes are dynamic features that evolve over space and time. Below we describe three prominent types of plumes, how they form, how they are characterized, and how we can detect them using AUV-mounted sensors.

\subsubsection{Hydrothermal Vent Plumes}

Hydrothermal vents occur on the sea floor at circulation zones near underwater plate boundaries, most often along plate spreading centers. In these regions, seawater seeps down into the earth's crust, undergoing chemical reactions as it is rapidly heated within the rock below before it is ejected back up through a sea floor hydrothermal vent and into the cold surrounding seawater. This chemical-filled vent fluid rises, reacts with its surrounding environment, cools, mixes, and spreads out horizontally at some distance above the sea floor to form a hydrothermal vent plume. This process is depicted in Fig. 6-1.

Hydrothermal vent plumes are characterized by the spatial extent of a plume's non-buoyant layer above the sea floor, which, according to [146], can extend tens to thousands of kilometers from the vent itself. Thus far, the most successful way to find the source of a hydrothermal vent plume is to first find the plume, and then track its chemical and physical signature back to its source. In particular, scientists examine temperature anomaly, particle content, water velocity, chemical tracers (iron, manganese, helium, methane, hydrogen sulfide), and bathymetric signatures in the water near potential hydrothermal vent sites to determine the presence of a plume and vent field.

A number of sensors that detect the aforementioned chemical and physical signatures are given in table 6.1

(see [147] and [148] for further sensor details). Many of these sensors can be mounted off-the-shelf onto an 


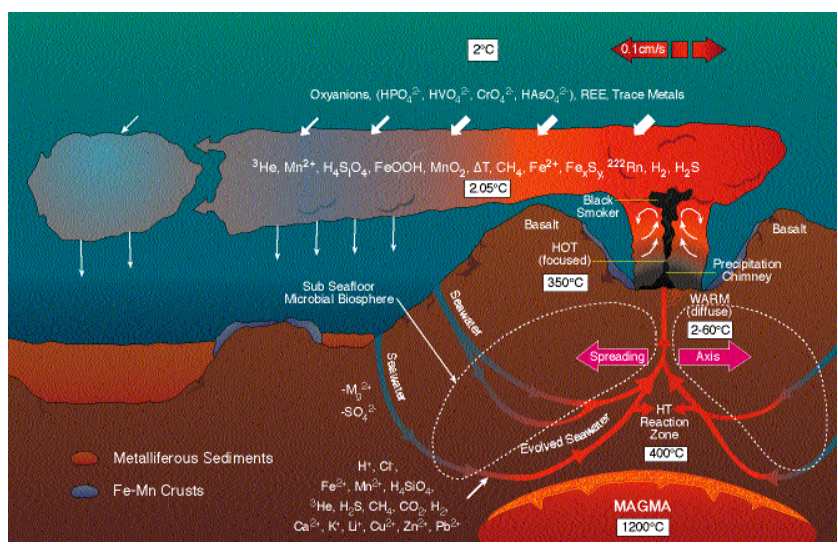

Figure 6-1: Formation of a hydrothermal vent plume. Image credit: [145].

Table 6.1: Sensors to detect hydrothermal vent plumes and sources

\begin{tabular}{|l|l|}
\hline Signature & Sensors \\
\hline \hline $\begin{array}{l}\text { Temperature } \\
\text { anomaly }\end{array}$ & CTD (Conductivity-Temperature-Depth) sensor \\
\hline Particle content & Optical sensors: transmissometer, nephelometer \\
\hline Water velocity & $\begin{array}{l}\text { Acoustic sensors: ADCP (Acoustic Doppler Current Profiler), } \\
\text { sidescan sonar, multibeam sonar }\end{array}$ \\
\hline Chemical tracers & $\begin{array}{l}\text { Optical sensors: SUAVE (System Used to Assess Vented } \\
\text { Emissions), ZAPS (Zero Angle Photon Spectrophotometer), eH } \\
\text { (redox potential) sensor }\end{array}$ \\
\hline Bathymetry & Multibeam mapping sonar, camera (still or video) \\
\hline
\end{tabular}

AUV, although some are still custom-made for oceanographic applications.

\subsubsection{Oil Spills}

There are two primary sources of oil input into the ocean: natural seeps from beneath the sea floor that account for about $50 \%$ of oil in the coastal ocean and oil spills (and oil runoff) from human activities (see Fig. 6-2). Since the methods for detecting oil seeps are not within the scope of this chapter, we address only oil spill characteristics here. When an oil spill results from human activities, the source location is often well known, and scientists need to know the spatial extent of the resulting oil plume in order to assess damage to the environment, flora, fauna, ocean-sourced food supplies, and coastal human populations. Although a large portion of oil rises to the surface during a spill event to form a slick, over time (on the order of seconds to years) the chemicals in oil react with the seawater and are consumed and broken down by microbes in the water, leading to an eventual fallout of the remains of the oil into a layer in which it is neutrally buoyant 


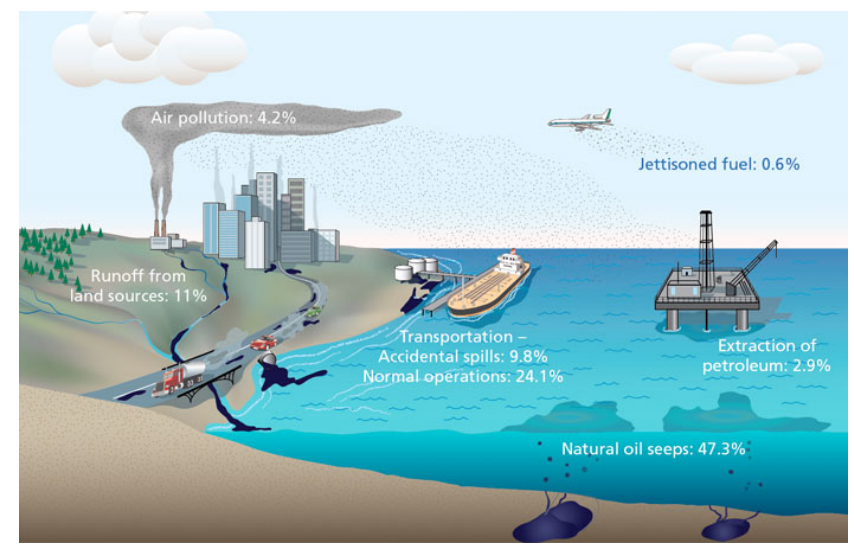

Figure 6-2: Sources of oil in the ocean. Image credit: Jack Cook, [149].

and/or into a pile on the sea floor, where microbes in the sediment further break down the oil. As evidenced in the work of [46], a significant amount of oil may be entrained in a neutrally buoyant layer well below the sea surface long after the surface slick has been dispersed and before total fallout occurs.

Oil spills vary widely in horizontal extent depending on the type of source supply (e.g., ship leak, broken well pipe on a drilling rig, shore runoff, etc.) and local flow characteristics. The underwater plume from the leaking MC252 Macondo well site in the Gulf of Mexico, for example, exhibited 5-day-old oil that had spread over 30 kilometers from the well head location when the team of [46] found and mapped the plume.

The residence time associated with oil in the ocean can vary from months to years, depending on the severity of the spill/leak. Seep oil in particular remains on the surface or suspended in the water column for anywhere from about 10 hours to 5 days before settling to the sea floor (see [149]). Thus, a similar time line is likely for some oil resulting from spills.

Finally, the best way to detect the presence of oil in the water is to analyze the hydrocarbon concentration of the water. There are a number of sensors that have been used to detect hydrocarbons in the water remotely, in a lab, and in situ. [150] have used thermal infrared sensors, laser fluorosensors, and radar to sense hydrocarbon concentration. Other techniques involve ADCP or Doppler Velocity Log sensors to record the currents and predict spreading direction of the oil, while a mass spectrometer is used to detect the hydrocarbons.

\subsubsection{Harmful Algal Blooms}

Harmful algal blooms differ from hydrothermal vent plumes and oil seeps and spills in that HABs do not have a source location feeding the plume (bloom). Instead, HABs are triggered when significant amounts of nutrients (nitrogen and phosphorous) and light are sustained in a region, resulting in an abundance of 
algal growth (blooming) that is often visible by the human eye as tiny red, green, orange, or brown particles (algae) floating in a thick layer near the surface of the water. Such areas are often classified as eutrophic zones along the coast, since nutrient runoff from land usage gets trapped in the relatively shallow and warm coastal waters, resulting in algal blooms. In such high concentrations, the toxins in some types of algae become lethal to marine organisms that consume them (and to people that eat the contaminated seafood), and can even result in a hypoxic zone due to the depletion of dissolved oxygen by the excess of algae.

Since HABs do not have a source, once the bloom is formed, it is transported largely by physical ocean processes such as coastal currents, wind, buoyancy, mixing, tides, and eddies. This transport can carry the bloom hundreds to thousands of kilometers. The vertical extent of the bloom is often on the order of tens if centimeters, making it a nearly $2 \mathrm{D}$ feature in space, spreading out horizontally near the surface or along the thermocline, covering 10-1000 kilometers in range (see [151]). The residence time of a given HAB varies widely based on nutrients, light, and algal life cycle.

There are a number of ways to detect and classify algae in a HAB, some of which are in situ, and some must be used on samples in a lab (see [151]). In situ sensors for HAB detection:

- Nutrient monitors

- Antibody probes (for a phosphorous-regulated protein)

- Flow cytometry

- Chlorophyll in vivo fluorescence (not ideal, as not all HABs contain chlorophyll)

- Nucleotide probes

- Quantitative polymerase chain reaction (PCR)

Other sensors for $\mathrm{HAB}$ detection:

- Microarray chip technology

- Electrospray ionization mass spectrometry

- Visual microscopic examination of water/biomass samples (a slow and tedious process)

\subsection{AUVs}

Having classified the primary plume types that we would like to detect and track with AUVs, we now move on to classify the abilities and traits of a variety of AUVs. Although this will not be a thorough classification 
of all AUVs, since there are many different commercial and made-in-house AUVs in the ocean community today, we aim to generalize a number of AUVs into categories that will allow us to best select a type of AUV to track a specific type of plume.

The most basic attributes to look at when comparing AUVs are speed, deployment duration (battery life), propulsion (active or passive), range of motion control, depth rating, navigation method, communication, hotel power load on board, autonomy system, hull shape, ease of retrofitting sensors, and what sensors it carries 'off the shelf'. See table 2.2.

To pair AUVs with a type of plume it is best suited to detect or track, we will consider the two primary classifications of AUVs: gliders and actively propelled AUVs. For long-duration deployments (days to months), the duration of gliders makes them the best type of AUV for the job. Multiple gliders distributed in a coordinated manner are also marginally sufficient to track a HAB advected by ocean currents, since the passive propulsion and resulting slow speed of gliders through the water are directly affected by the currents as well, pushing the gliders in the same direction as the plume is advected (see $[41,42])$. For very deep missions that are time-dependent (achievable in or requiring short mission time, as in hours or days), involve plumes that are highly time-variant, or require tracking a plume to its source against the local currents, actively propelled AUVs are the better choice despite their shorter deployment duration. This includes quickly detecting, tracking, and mapping an oil spill plume as in [46], as well as searching for hydrothermal vents near the sea floor while the plume changes location due to deep currents as experienced by [39]. In these cases, actively propelled AUVs may be used solo, or in a coordinated fleet if a meso- or large-scale plume must be mapped as the plume advects with the changing currents. Actively propelled AUVs would also be useful in quickly surveying the plume extent of a HAB in the horizontal plane, providing more of a snapshot of the HAB position as the $\mathrm{AUV}(\mathrm{s})$ is(are) deployed from day to day (and retrieved to recharge overnight).

\subsection{Plume Tracking Methods}

With a knowledge of the capabilities of various AUVs and the characteristics of underwater plumes, there are a variety of approaches to take towards autonomously and adaptively detecting and tracking plumes with AUVs. Here we will look at the plume detection and tracking methods of a number groups and present the preliminary methods proposed by our group. 


\subsubsection{Related Literature}

Tracking the general motion of HABs with AUVs (gliders, in particular) has been explored by [41] and [42]. [41] used a regional ocean model off of the coast of California to forecast the advection of an imaginary HAB that is tagged by actual Lagrangian drifters in the region, while [42] look for "hotspots" of high-concentration $\mathrm{HAB}$ patches using satellite and high-frequency radar data sets. Both use the frequently-updated remote sensing information and in-water drifter positions to tag the HABs, and then run mission-planning algorithms on a shore- or ship-side computer to update waypoint paths every few hours for gliders deployed in the area to actively track the (imaginary) $\mathrm{HAB}$ as it advects. Although these are good approaches to HAB tracking, the use of remotely sensed data or models requires extra time, computational power, and hard drive storage space that is not available on board gliders, and often not available on actively-propelled AUVs either, requiring connection of the AUVs to some shore- or ship-side computer to update the models and AUV paths.

The works of Farrell et al. [141] and Pang \& Farrell [142] are very significant to this field, as they employ actively-propelled AUVs to detect and track man-made plumes of Rhodamine dye back to their sources in a relatively constant flow field within the bottom boundary layer of coastal waters ( $<30$ meters deep). The REMUS AUV used by the group in field experiments was equipped with an ADCP unit to record the currents through the water column, a sensor that could detect trace concentrations of the Rhodamine dye for tracking the plume, and on board autonomy algorithms that perform lawnmower pattern surveys in the horizontal plane at constant altitude above the sea floor until the sensors detect the dye plume, at which point the AUV switches autonomously into plume-tracking mode. In plume-tracking mode, it uses a combination of realtime current data and dye concentration data it has collected to determine the direction of travel with the highest probability of finding the dye source, zigzagging across the plume in the horizontal plane until it has determined the source location. Pang [143] takes this one step farther using Artificial Potential Field methods in simulation to improve upon source localization algorithms with the application of tracking hydrothermal vent plumes to find the vent locations.

The work by Jakuba et al. [39] takes a somewhat different approach using a towed instrument package with a CTD and optical backscatter (OBS) sensor to detect the non-buoyant plume emitted from hydrothermal vents at the Juan de Fuca Ridge, and then deploying WHOI's ABE AUV to localize the hydrothermal vents. The vent localization was done as nested lawnmower surveys of successively finer resolution using a combination of eH, temperature, depth, and OBS sensors, multibeam bathymetric mapping, and photos for creating photomosaics of the sea floor vent sites. The increased efficiency that would result from automating the nested surveys and incorporating current measurements into vent localization is also discussed in the pa- 
per, and the resulting autonomous nested survey technique for sampling and mapping feature-rich areas in hydrothermal vent fields is discussed by Yeorger et al. in [152].

Camilli et al. [46] took a reconfigurable, but not autonomous, zigzagging plume tracking approach to detect and track the path of the underwater oil plume from the leaking Macondo well in the Gulf of Mexico using WHOI's Sentry AUV. Sentry's mass spectrometer gathered hydrocarbon concentration data along its path, sending snippets of data via acoustics back to the shipboard AUV operator for inspection in case the AUV mission needed reconfiguring to keep the vehicle in contact with the plume. Towed instruments and instruments cast over the side of the ship to collect further data and water samples also provided data to augment and verify the data collected by Sentry. This is one example in which, if more on-board AUV autonomy were employed to adapt the AUV's motion to its sensor readings, much mission planning and AUV redirection on the part of the AUV operators and scientific crew could have been eliminated, and AUV excursions kilometers from the plume could have been significantly reduced, saving time and battery power. The advantage to the approach taken by Camilli et al. was that it was reliable to implement and execute over the very short time frame they had available for mission preparation, deployment, and recovery, even if the signal from the plume were not nearly as clear as it was in this case. The autonomous algorithms they had available and new eH sensor they were using had not been rigorously tested to be satisfactorily reliable with potentially very weak hydrocarbon signals in such a pressing situation.

Finally, Cannell et al. [144] proved autonomous and adaptive plume mapping and boundary tracking possible using a single AUV to map the outflow plume of cooling water from a nuclear power plant. The AUV could adaptively zigzag across the plume and along the plume boundary.

\subsubsection{Our Approach}

We propose to employ a behavior-based autonomy architecture on board AUVs (both actively-propelled and gliders) in order to make the adaptation of the AUVs' motions to the environment fully autonomous. Preliminary testing of autonomous and adaptive environmental feature tracking has already been successfully completed on a number of models of actively propelled AUVs (see [3]), but the power restrictions on board gliders have prevented the use of fully on-board autonomy thus far. A number of groups (including ours) are currently looking into this problem, and we expect to see some fully autonomous gliders tested in the next few years as more autonomy systems migrate onto low-power embedded computers.

It is easiest to attempt plume tracking with a single AUV due to the relative simplicity of deploying and monitoring only one vehicle; however, it may be physically impossible to collect a synoptic data set 
representing a meso- or large-scale plume with only one AUV due to speed, battery life, and other constraints. On the meso- and large- horizontal spatial scales (tens to hundreds of kilometers, or more) frequently covered by dynamic underwater plumes, it is reasonable to assume that the use of multiple AUVs with the ability to autonomously coordinate their own motions through acoustic (or radio-frequency, if absolutely necessary) communication methods would be advantageous.

With fully autonomously-controlled AUVs in mind (both solo and coordinated in a network), we discuss various approaches to tracking plumes with different characteristics below. We assume that non-buoyant plume layers can be approximated as $2 \mathrm{D}$ when plume tracking in the horizontal plane.

\section{Non-buoyant layer plume detection.}

The search for a non-buoyant layer plume consists of motion in the horizontal plane coupled with depth excursions along the vertical axis. The vertical motion is a yo-yo pattern to determine the depth range in which the non-buoyant plume is floating. This allows all types of AUVs (gliders and otherwise) to be suited to this task. The horizontal motion of an AUV may be a circular pattern centered around the source location (if known) to determine the dominant direction that the plume has spread in, or it may be a rectangle, strait line, or lawnmower survey pattern over an area where sources are predicted to be nearby (or if there is no source, as in $\mathrm{AAB}$ ) in hopes of detecting a plume.

\section{Plume source discovery.}

To find an unknown source location after a plume is detected, detect and track the non-buoyant plume back to its source. There are a number of approaches to this which are best suited to actively propelled AUVs, with the ability to make headway swimming against the currents and easily change heading if necessary. Gliders are much slower to travel and maneuver, with a maximum speed through water of under $0.5 \mathrm{~m} / \mathrm{s}$ (and often $<0.3 \mathrm{~m} / \mathrm{s}$ ), making little or negative headway against currents any greater than about $0.5 \mathrm{~m} / \mathrm{s}$.

Assuming an AUV has a current measuring device such as an ADCP on board, once the plume is detected, the AUV may attempt to swim directly upstream against the current towards the presumed source location. However, it is likely that the plume's meandering motion from time-dynamic currents results in an indirect path back to the source, requiring the AUV to perform a horizontal zigzagging motion to remain in contact with the plume as it follows the plume upstream. If the plume is relatively skinny, $\mathrm{O}(1 \mathrm{~km})$, and the currents are largely constant in direction, as in [141] and [144], it is possible to use a single AUV to track the plume

back to the source, or map the plume. A more wide-spread or patchy plume may require multiple AUVs to 
most efficiently track upstream while maintaining contact with the plume. Another source discovery method, mentioned by [39], tracks the plume in the direction (horizontally) of increasing vertical current velocity in the non-buoyant layer. The active source, if it is on or near the sea floor, will spew a vertical jet of fluid and/or particles, resulting in the largest vertical currents at the position above its location. Again due to the potential for highly time-dynamic currents advecting a non-buoyant plume over a relatively short time scale (hours), an autonomously coordinated network of AUVs should be deployed to improve plume sampling coverage and work together to track the plume to its source, avoiding the spatial and temporal aliasing problem experienced by [39] while tracking hydrothermal vent plumes with their single AUV over hours-long missions.

\section{Plume boundary tracking.}

For the times we want to track just the boundary position of a plume (assuming it is $2 \mathrm{D}$ in the horizontal plane), there are two approaches we can take, depending on the spatial extent of the plume in the horizontal plane. In these cases, the vertical yo-yo motion of a glider is unnecessary, its means of locomotion make it slow, and most gliders cannot power the acoustic communication hardware necessary for multi-AUV missions without drastically reducing deployment duration. Thus, we propose the use of an autonomously coordinated network of actively-propelled AUVs for plume boundary tracking, much like that discussed in Appendix B ( [4]) and using front boundary tracking methods described in Chapter 5.

As noted above, a single actively-propelled AUV can track a plume boundary by zigzagging across the entire plume width, if the plume is relatively skinny, $\mathrm{O}(1 \mathrm{~km})$ wide. If a plume is much larger in horizontal extent, more of a blob-shape, or highly dynamic in time, it is most efficient to employ multiple coordinated AUVs to find and track the plume boundary. This involves autonomy on both a 'global' scale and a 'local' scale, as illustrated in Fig. 6-3. The global scale would ideally entail multiple AUVs communicating and exchanging data using acoustics in real time to autonomously coordinate their search patterns to find the plume boundary, and then re-arrange their positions along the boundary of the plume to maintain optimal spacing between AUVs and collect a synoptic data set around the entire plume boundary. On the local scale, each AUV will track the plume boundary in its immediate vicinity by zigzagging in and out of the plume across the boundary, using adaptive autonomy to adjust its direction of travel in real time as the edge of the plume shifts in space and time, similar to the method used by [144]. In order to keep adaptive and autonomous plume tracking robust to 'holes' in the plume and small variations in the local plume boundary position, each AUV would keep track of "inside-of-plume" and "outside-of-plume" samples (a boolean indicator) within some temporal or spatial range from its current position, averaging over the samples to determine whether it 


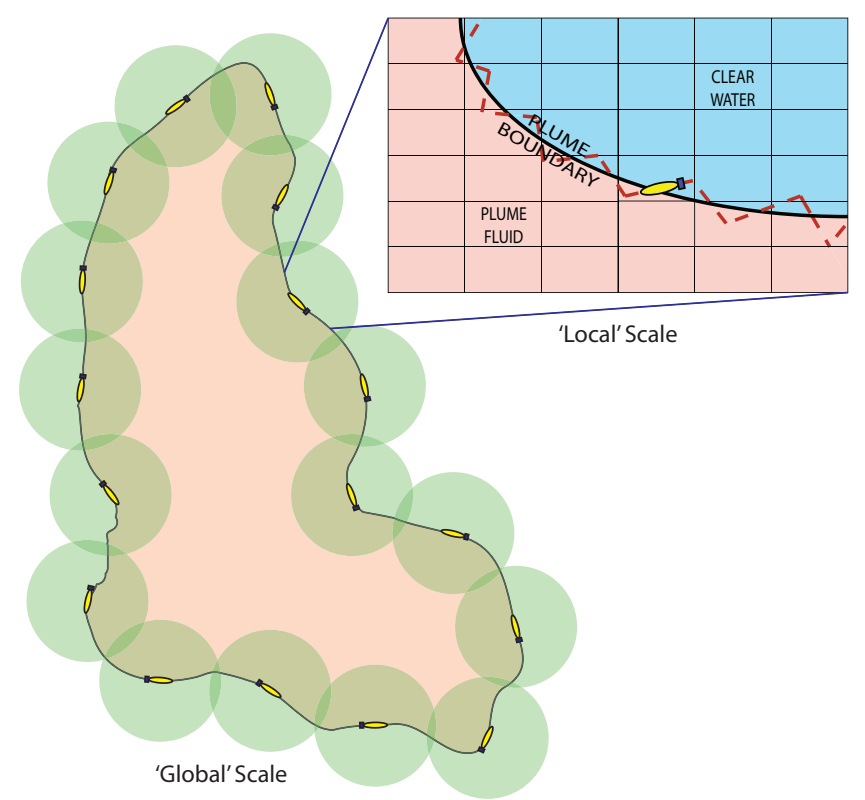

Figure 6-3: Concept for multi-AUV coordination and tracking of a plume boundary on the 'global' scale, and 'local' scale tracking of the plume boundary using a zigzag pattern across the boundary in the horizontal plane. The circles are range rings around each AUV, specifying the range within which all samples collected by the AUV may be considered current measurements of the plume (all samples within the characteristic time and spatial scales of the dynamic plume).

has most likely left the plume (and should reverse its travel direction), or is still in the plume. Preliminary details of the setup, implementation, and logistics of this multi-AUV plume boundary tracking system are described in [4] and Appendix B.

\subsection{Conclusion}

It is relatively inefficient to go to sea to tow instruments from a ship in extensive survey patterns in hopes of detecting the signature of an underwater plume. While it is more efficient to deploy ROVs or pre-programmed AUVs for this purpose, an ROV requires constant supervision and can only travel as far and deep as its tether will reach, while a pre-programmed AUV must transmit data to the shore lab or ship lab for extensive data analysis by the scientists before refining the AUV's search pattern. Thus, in this chapter we gather and present information on the plume characteristics of hydrothermal vent plumes, oil spills, and harmful algal blooms, and pair the various types of plumes with types and abilities of AUVs that we believe would be most efficient to track each plume type or find a plume source. With this plume-AUV pairing knowledge, we have determined that the most efficient approach to dynamic plume and plume source tracking is to use a fleet 
of autonomously-coordinated, actively-propelled AUVs, each with an individual on-board autonomy system that allows for autonomous adaptation of the AUV's motion to changes it senses in the local environment (e.g., hydrocarbon concentration drops as an AUV swims out of an oil plume, so the AUV autonomously changes heading to swim back into the plume). The multi-AUV approach to plume tracking, with autonomous adaptation of AUV motion to other AUVs and to changes in the environment, offers the opportunity to efficiently collect spatiotemporally synoptic data sets of plumes and plume sources that are essential to getting the most out of limited at-sea time and to better understanding and monitoring these ocean features.

Plume tracking methods conclude the extent to which we have investigated complex autonomous and adaptive ocean feature tracking methods on board AUVs. The methods covered throughout this thesis will be summarized in Chapter 7, as well as some potential future directions for extending this work. 


\section{Chapter 7}

\section{Conclusion \& Future Directions}

The goal of this work was to develop a method of adaptively and autonomously tracking hydrographic features using AUVs, based solely on the data that the AUVs can collect and process on board, in real time. The constraints imposed upon the development of this method were to use AUVs to sample and track features 1) adaptively to account for the dynamic nature of hydrographic features, 2) collaboratively between AUVs (and other marine platforms) to collect data sets exhibiting improved synopticity for feature detection and classification, and 3) autonomously such that the AUVs determine the spatiotemporal positions or boundaries of the features, to more efficiently detect and track the features with as little human intervention as possible.

Chapter 1 gave an introduction to the work presented in this thesis, including motivation, constraints, and the proposed approach to autonomous and adaptive oceanographic feature sampling and tracking that is presented from concept to implementation and field results throughout the rest of this thesis.

Chapter 2 provided the technical and oceanographic background for the thesis. It explained some of the available AUV technologies, the challenges of working with AUVs in the ocean environment, and the concept of characteristic spatiotemporal scales of oceanographic features. It also looked at past and current methods of oceanographic feature tracking from the literature to further motivate the work in this thesis.

Chapter 3 introduced the concept of Autonomous and Adaptive Environmental Assessment (AAEA) of oceanographic features using AUVs. This concept was then applied to thermocline tracking with AUVs as a proof-of-concept taken from theory to implementation. Thermocline tracking results were given for multiple field experiments.

Chapter 4 described the Internal Wave Detection Experiment in the Tyrrhenian Sea in 2010. This experiment was designed to showcase the use of autonomous and adaptive thermocline tracking coupled with multiple autonomously coordinated AUVs to capture the signals of any passing internal waves. The results 
characterizing the detected internal waves were also presented.

Chapter 5 explored autonomously and adaptively detecting and tracking underwater fronts in detail. These features are often significantly complex and dynamic in both horizontal space and time. $2 \mathrm{D}, 3 \mathrm{D}$, and multi-AUV front tracking behaviors that have been developed for this work and tested in virtual experiments were described, and results from the tests in a simulated MSEAS ocean model environment of the MidAtlantic Bight region were presented.

Chapter 6 explored concepts and approaches for autonomously and adaptively detecting and tracking various types of underwater plumes.

This thesis has applied the process of Autonomous Adaptive Environmental Assessment and Feature Tracking on board AUVs to the sampling and tracking of a number of oceanographic features in the dynamic ocean environment. AAEA is a process by which an AUV autonomously assesses the hydrographic environment it is swimming through in real time. This assessment is essentially the detection of hydrographic features of interest and leads naturally to the subsequent active/adaptive tracking of a selected feature. The detection-tracking feedback loop setup with AAEA currently aims to use solely an AUV's self-collected hydrographic data (e.g., temperature, conductivity, and/or pressure readings), along with a basic quantitative definition of an underwater feature of interest, to detect and track the feature. Feature tracking must be both autonomous in the sense that the AUV operator is not involved in guiding the vehicle outside of commanding it to "track feature X," and adaptive in the sense that, as a dynamic feature evolves over space and time, the AUV will recognize any changes and alter course accordingly to retain data coverage of the feature.

The features explored for AUV sampling and tracking in this thesis included thermoclines, internal waves, fronts, and plumes, in increasing order of complexity. The feature detection and tracking methods developed, using both individual and multiple AUVs, have provided increased efficiency and synopticity in sampling, while reducing the amount of human guidance necessary to collect the desired data sets.

Chapters $3 \& 4$ presented methods of autonomous and adaptive thermocline tracking and internal wave detection with multiple AUVs, which have been validated successfully in field experiments. The Internal Wave Detection Experiment in particular has also contributed new oceanographic findings to the scientific community, characterizing internal waves in the Tuscan Archipelago Basin-in the Tyrrhenian Sea west of Italy-that were previously unknown to the researchers studying the area. Chapter 5 discussed a novel method for front tracking, including autonomy behavior implementations for $2 \mathrm{D}, 3 \mathrm{D}$, and multi-AUV autonomous and adaptive front tracking. Virtual experiment results from testing the front tracking behaviors reveal a general improvement in efficiency and increase in along-front sampling density over conventional preplanned- 
path methods, as well as a reduction in distance traveled that did not track along the front. The only downfall to the $2 \mathrm{D}$ front tracking behavior as it stands is the sensitivity to small-scale $(<3 \mathrm{~km})$ temperature variations that was observed in the higher-resolution ocean model it was tested in. Chapter 6 researched various types of underwater plumes and devised new concepts and methods that could be implemented to track them in the future, including the use of multi-AUV front tracking methods that were developed in Chapter 5 to synoptically track along plume boundaries dynamic in space and time.

\subsection{Looking Ahead}

There are many extensions of this research as AUVs become more widely used by the oceanographic community. The first step is to further improve the $2 \mathrm{D}$ front tracking behavior by adding front detection and front temperature selection using the temperature at the peak azimuthal temperature gradients around a circular initial loiter. The next objective would be to decrease the sensitivity of the $2 \mathrm{D}$ front tracking behavior to small-scale changes in temperature by including active binning or time-averaging techniques to smooth the data in real time, essentially applying a low-pass filter to the raw data, and by tuning the front tracking configuration parameters (zigzag amplitude and front intersection angle) to optimize performance according to the data resolution available. Then the front tracking with smoothing could be re-evaluated in virtual experiments using a high-resolution model (say, a $1 \mathrm{~km}$ resolution ocean model superimposed with $1 \mathrm{~m}$ resolution 'noise' data taken from AUV-collected field observations of temperature). Once the tuned front tracking behavior with smoothing has been validated in virtual experiments, it will be immediately ready for field testing on a thermal front using any actively-propelled AUV with a CTD and decent navigation. Field testing of the multi-AUV follow-the-leader behavior should also be done at this stage, both with and without front tracking behaviors running simultaneously. There is also room for more research into implementing 3D, multi-AUV front tracking with AUVs distributed throughout the water column to better characterize the interface between the two water masses that meet at the front. In regard to plume tracking, some groundwork has been laid here for autonomous and adaptive plume tracking behavior development based on the methods presented in this thesis, but there is a good deal of development, evaluation, and re-evaluation to be completed before these or related plume tracking methods can be fielded with confidence.

Finally, there are a multitude of other features that can be sampled more efficiently and synoptically with AUVs using the foundational methods and algorithms developed throughout this thesis. The use of temperature as the tracer of choice here can readily be exchanged for other tracers such as salinity, sound speed, density, chemical concentration, dissolved oxygen concentration, flow velocity, or even bathymetry, to 
name a few. In the case of thermocline tracking, the same algorithms have already been successfully tested in tracking the 'acousticline,' and it is up to future AUV operators and scientists to find creative new ways to put these feature tracking methods and algorithms to use to improve their oceanographic sampling techniques. 


\section{Appendix A}

\section{MSEAS Integration}

\section{A.1 LAMSS-MSEAS Interface}

This appendix describes the interface between environmental sensors on board a simulated AUV in the MIT Laboratory for Autonomous Marine Sensing Systems (LAMSS) AUV Autonomy Simulator and the Multidisciplinary Simulation, Estimation, and Assimilation Systems (MSEAS) ocean models.

\section{A.1.1 Virtual Experiment Environment}

The LAMSS AUV Autonomy Simulator allows the user to run virtual AUV experiments using AUVs running autonomy behaviors with a variety configurations using acoustic communications (or a simulation thereof) and sensor models. The MSEAS group creates, develops and utilizes physics-driven numerical models of dynamic oceanographic environments based on data from current and historical in situ and remotely sensed measurements. These models and data are used for ocean forecasting and nowcasting, dynamical diagnostics, and as environments for simulations and virtual experiments that benefit from incorporating a dynamic ocean model to verify performance. Thus, the combined LAMSS-MSEAS interface, which allows virtual AUV experiments to be tested in a dynamic ocean model environment, is shown in Fig. A-1.

The boxes marked in red represent the simulated processes. Most of the simulated processes use the uField toolbox, which contains a number of tools for supporting multi-vehicle missions where each vehicle is

Portions of this appendix are reprinted, with permission, from A. Balasuriya, S. Petillo, and A. Yaari, "LAMSS-MSEAS Interface Control Document," 2013. Unpublished. 


\section{Virtual Ocean Environment}

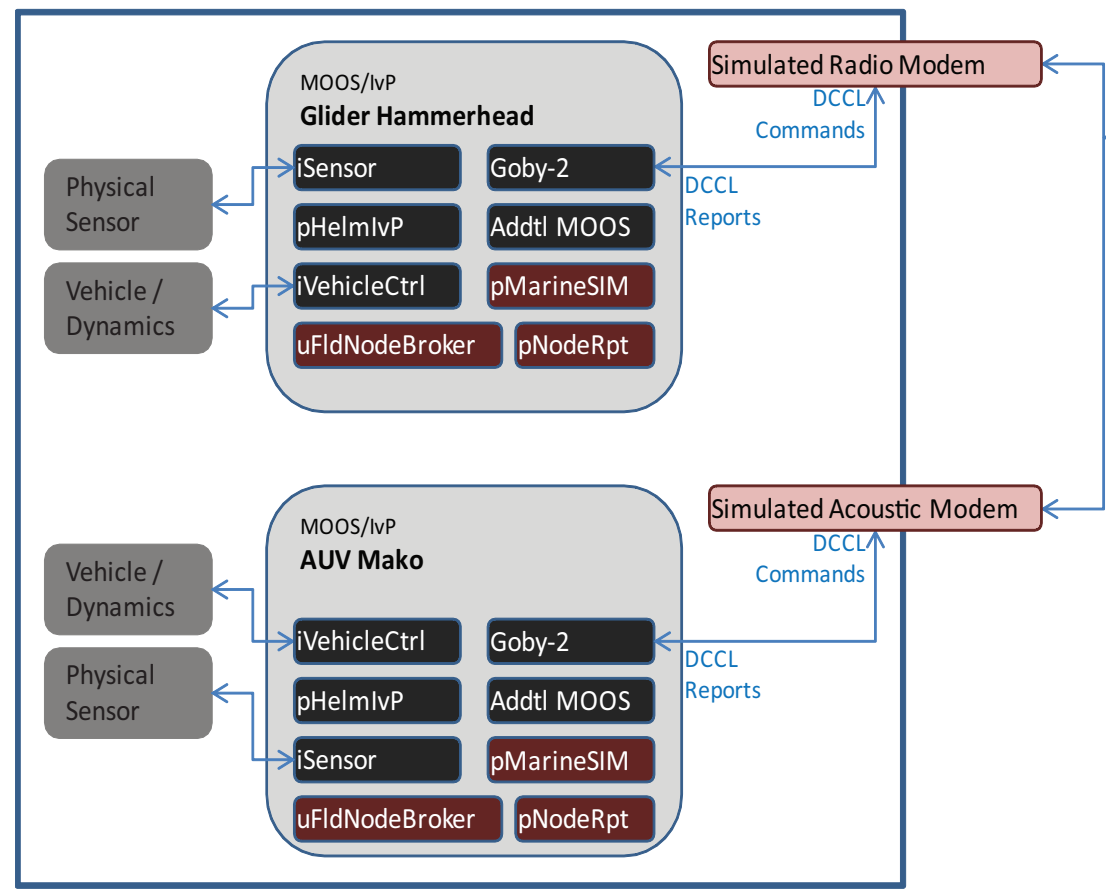

Topside

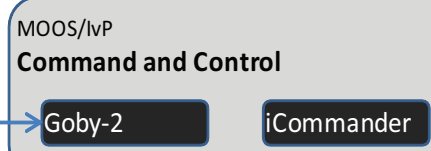

Environmental Model

Numerical Ocean Model

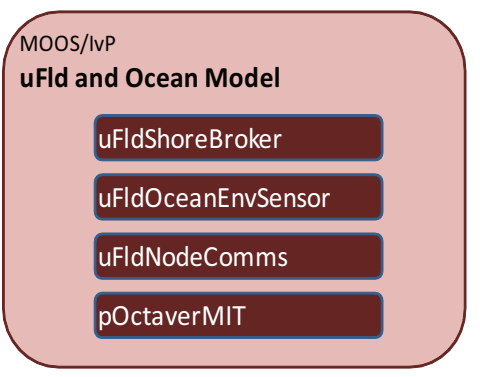

Figure A-1: LAMSS-MSEAS simulator interface.

connected to a shoreside/topside community. In Fig. A-1, AUV simulator components are shown in the left and shoreside/topside components are shown on the right.

The iSensor module in Fig. A-1 represents the software interface with a physical sensor in a real vehicle and with a simulated sensor in a simulated vehicle. In virtual experiments, iSensor requests sensor data from the MSEAS model using uField tools as illustrated in Fig. A-2. In the LAMSS simulator, two instances of the iSensor module are iCTD_OceanModel and iADCP_OceanModel, which stream data for the CTD and ADCP respectively.

The messages being passed between modules on a simulated AUV to create the LAMSS-MSEAS interface include a request message from iCTD_OceanModel or iADCP_OceanModel on the AUV to obtain the environmental characteristics, SIM_OCEANENV_REQUEST, which is re-packaged by uFldOceanEnvSensor with a unique request identification number and the most recent time and AUV position into the SIM_OCEAN_MODEL_REQUEST message. The SIM_OCEAN_MODEL_REQUEST is then sent to pOctaverMIT (Fig. A-3), which queries the MSEAS ocean model for the ocean characteristics at the time and location requested through an Octave interface (getmseas_octave_new.m and readmseaspe_octave.m), resulting in the reply message, SIM_OCEAN_MODEL_REPORT, containing the ocean characteristics along 


\section{Virtual Ocean Environment}

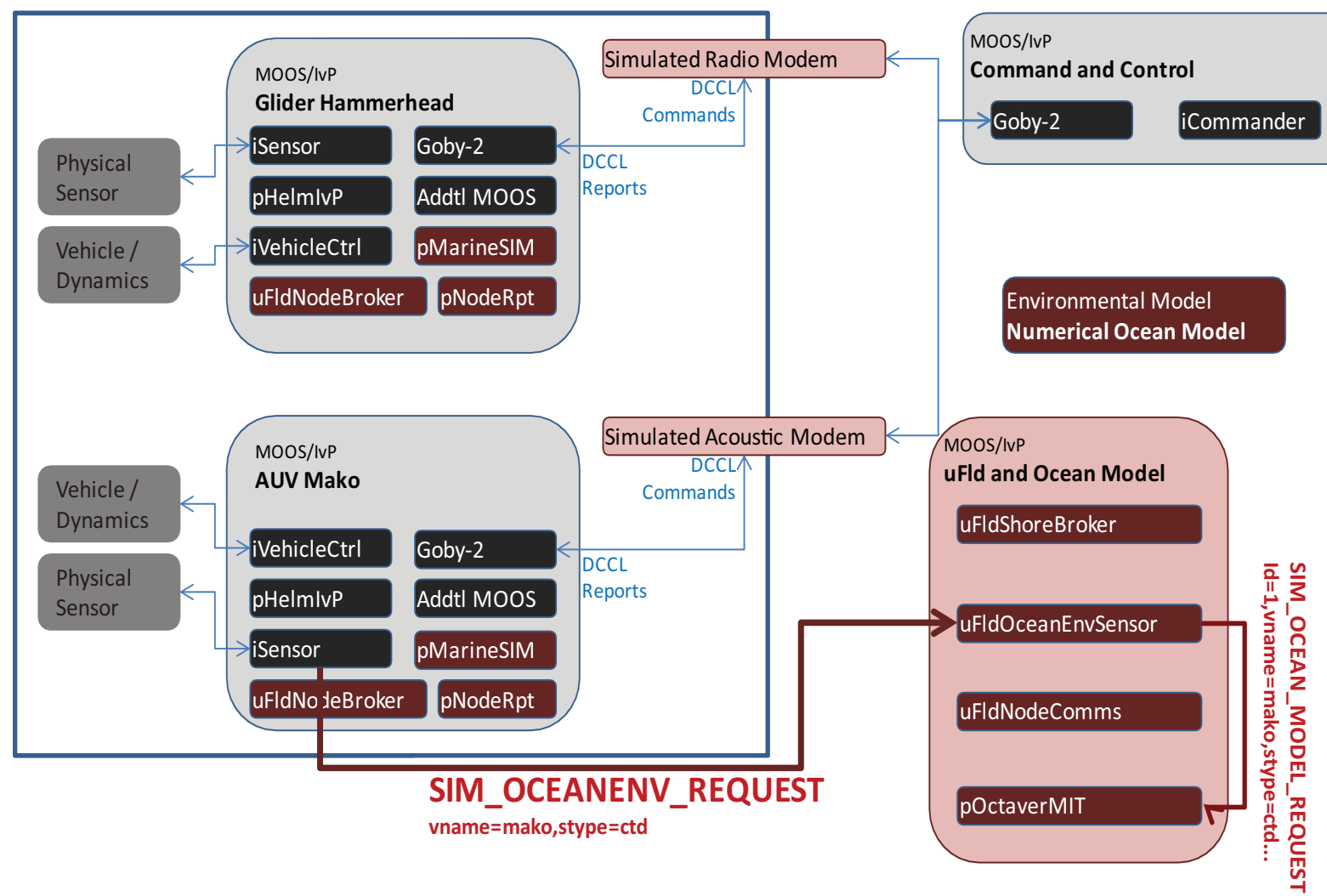

Figure A-2: iSensor request for data from shoreside/topside.

with the information from the request message. This reply is then consumed by uFldOceanEnvSensor (Fig. A-4), which strips the unique ID number and transfers the environmental data back to iCTD_OceanModel or iADCP_OceanModel as SIM_OCEANENV_REPORT. The contents of SIM_OCEANENV_REPORT is then used by various modules and behaviors on the AUV. The aforementioned messages and modules are detailed below.

SIM_OCEANENV_REQUEST = "vname=vehicle name, stype=sensor type"

vname - The name of the underwater vehicle

stype - Sensor type (e.g., for CTD data stype=ctd or for ADCP data stype=adcp)

SIM_OCEAN_MODEL_REQUEST $=$ “id=..,vname $=\ldots$, stype $=\ldots, \mathrm{x}=\ldots, \mathrm{y}=\ldots$, lat $=\ldots$, lon $=\ldots$, depth $=\ldots$, time $=. . . "$ 


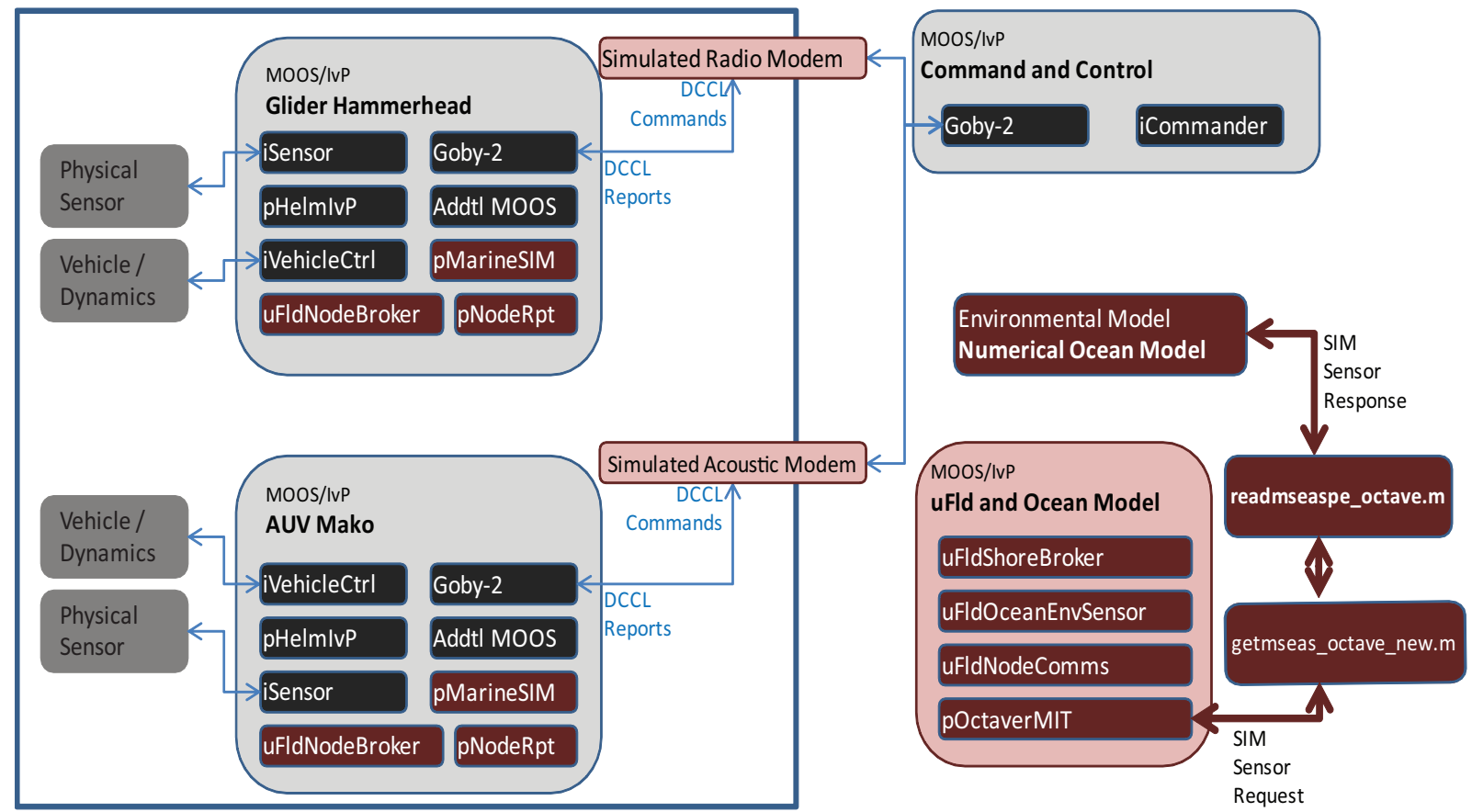

Figure A-3: pOctaverMIT requests data from MSEAS through getmseas_octave_new.m and readmseaspe_octave.m Octave scripts.

id - Request ID

vname - Requested vehicle name

stype - Type of sensor, e.g., CTD or ADCP

$(\mathrm{x}, \mathrm{y})$ - Local coordinates of the data requested

(lat,lon) - Global coordinates of the data requested

depth - Depth of the data requested

time - Time at which data is requested

pOctaverMIT (a derivative of pOctaver by Arjan Vermeij at CMRE, Italy) is used to execute Octave scripts (M-files) based on messages passed through the MOOS database in the LAMSS AUV Simulator. In this case, pOctaverMIT is configured to read the MSEAS ocean model CTD/ADCP data at the position and time requested by the AUV, as shown in Figs. A-2 and A-3. pOctaverMIT initializes the paths to the MSEAS ocean model NetCDF files containing the environmental data, sets the MOOS variables that the Octave script will require as input and output, sets the path to where the Octave script resides, and sets the name of the function in the script. 


\section{Virtual Ocean Environment}

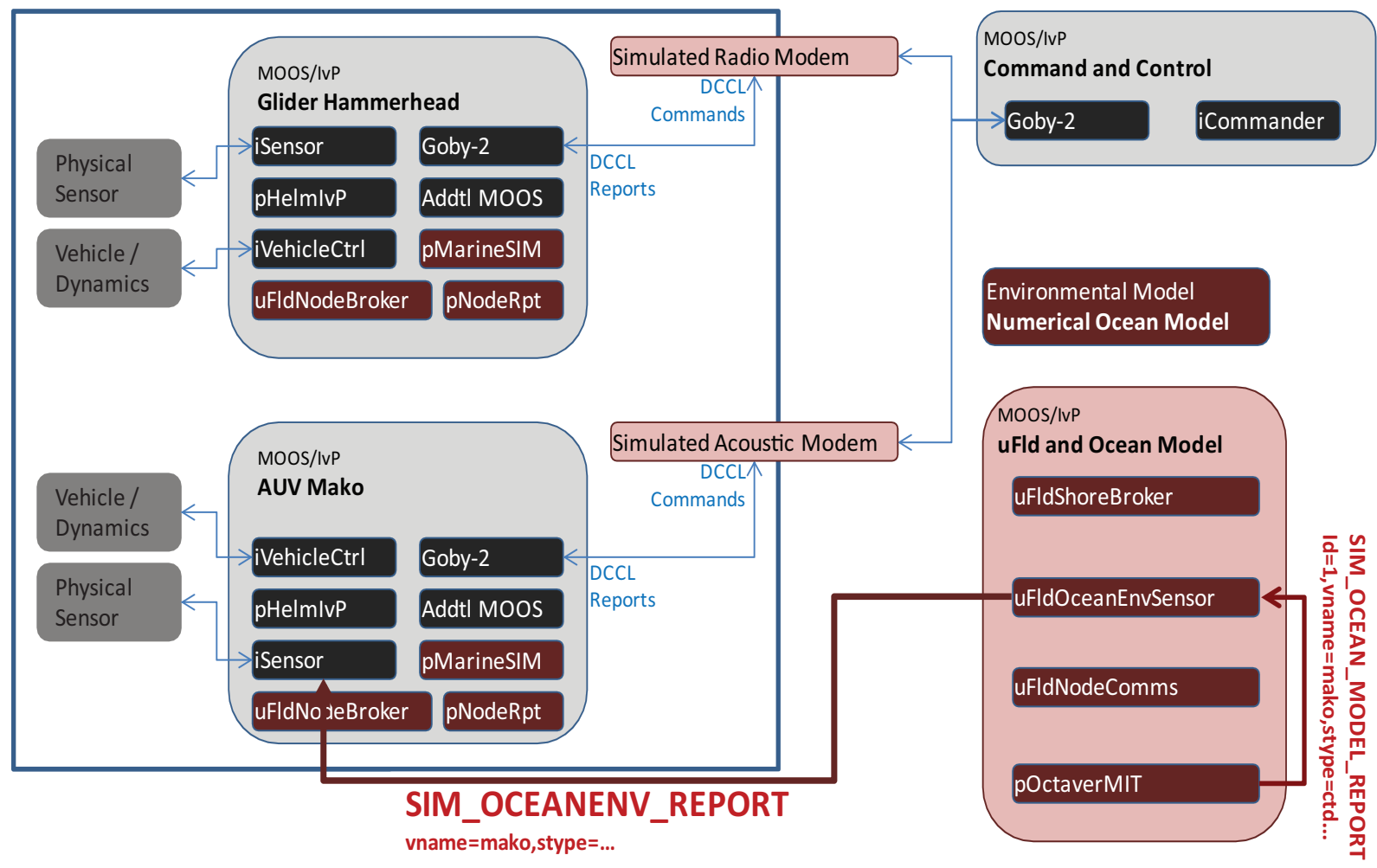

Figure A-4: iSensor receiving sensor data from MSEAS using uField tools.

The pOctaverMIT configuration for querying MSEAS models is as follows:

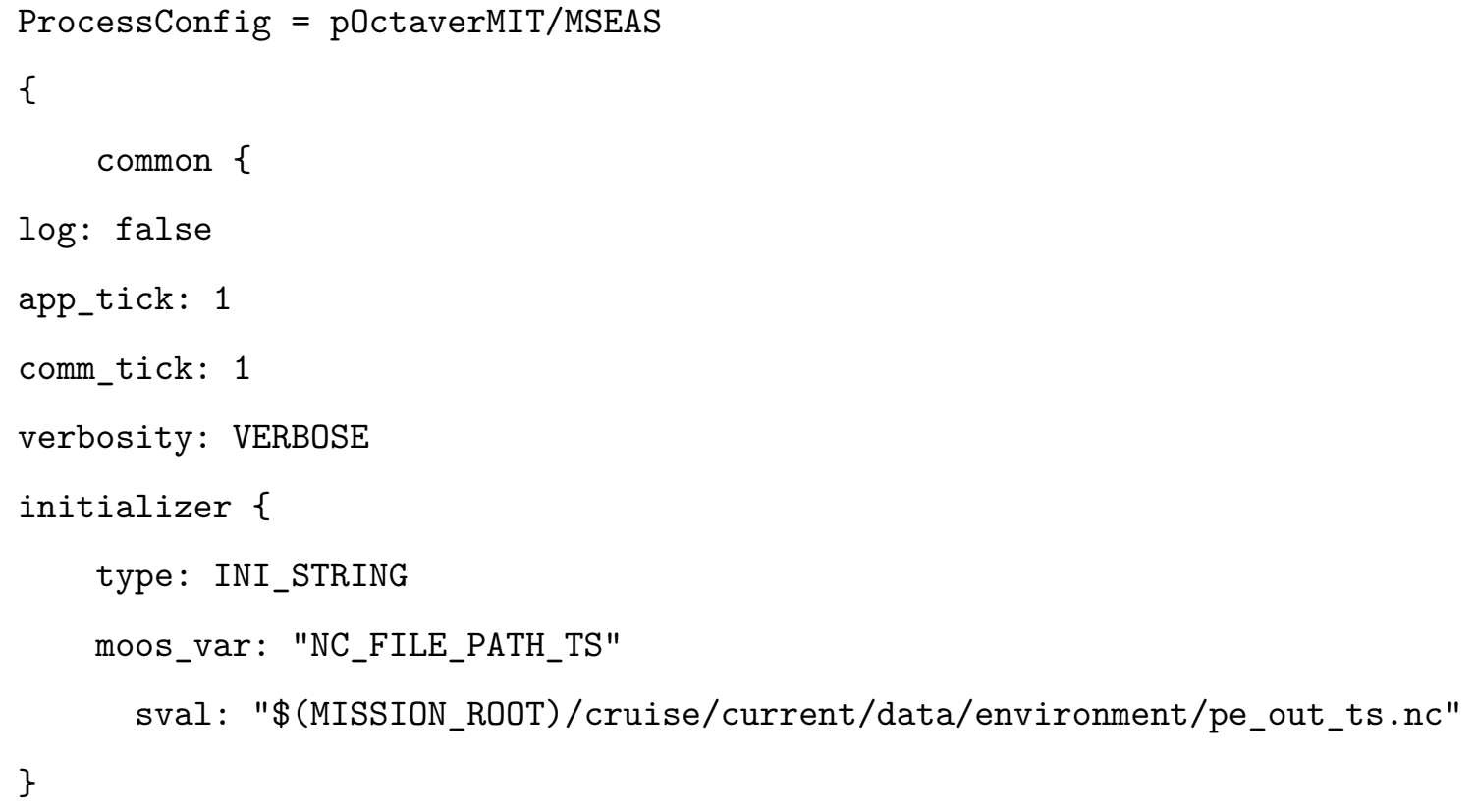




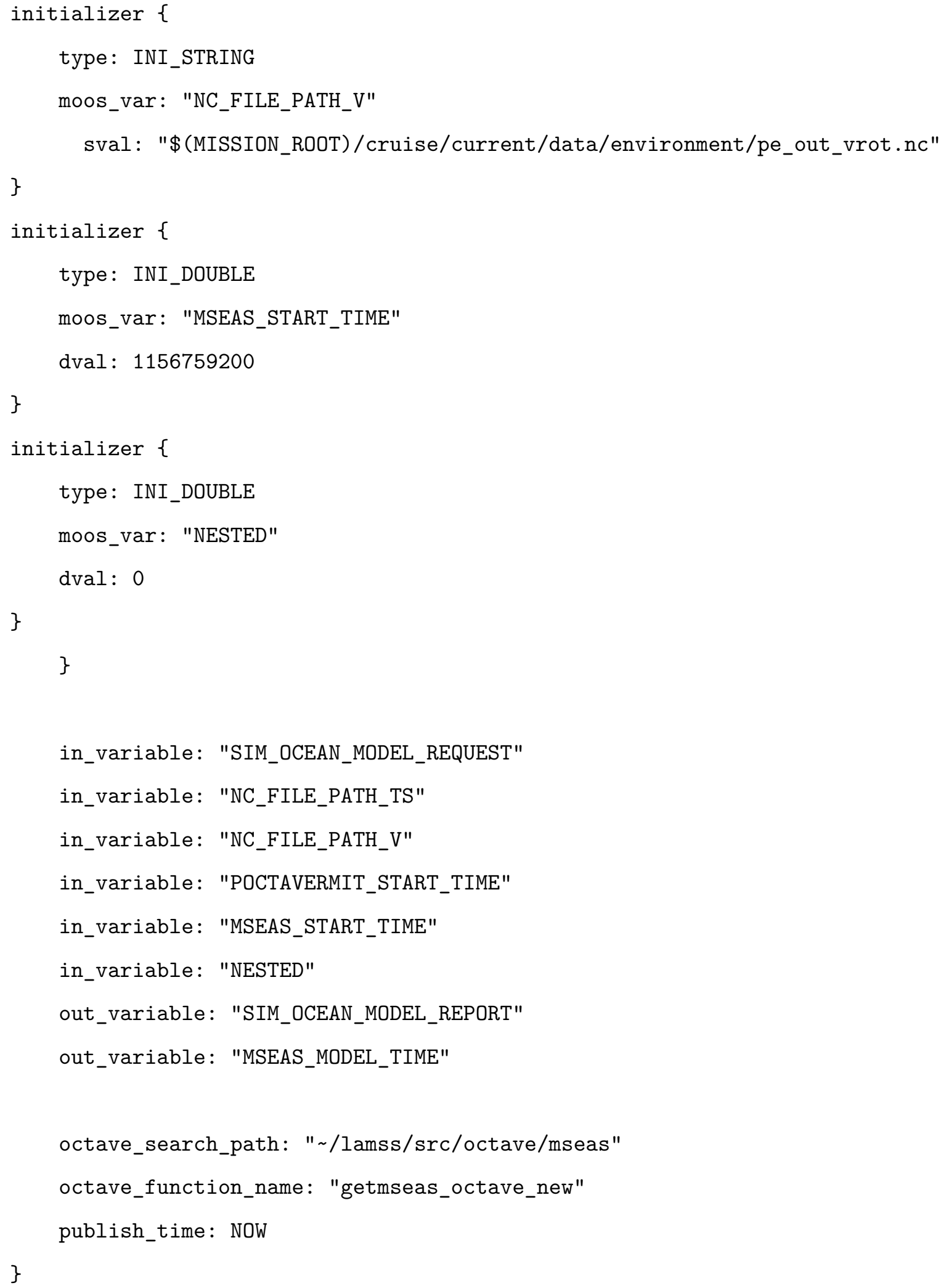


Initialization (initializer) $\&$ input variables (in_variable):

NC_FILE_PATH_TS - Path to the MSEAS model NetCDF file containing temperature $\&$ salinity data

NC_FILE_PATH_V - Path to the MSEAS model NetCDF file containing 2D or 3D current data

MSEAS_START_TIME - Desired UTC start time within the model time range (e.g.,

1156759200; if out of the model range, it will be automatically shifted in time until it is within), or 0 to start at the first available time slice of the model, or -1 to set the start time in the model based on the current actual time

NESTED - Are we using nested MSEAS models? $1=y e s, 0=$ no

\section{Other input variables (in_variable):}

SIM_OCEAN_MODEL_REQUEST - String containing the sensor type and AUV position and time that the ocean model data is being requested for. See configuration above.

POCTAVERMIT_START_TIME - Time pOctaverMIT started running, used to shift the start time of the virtual experiment to within the temporal bounds of the MSEAS model.

\section{Output variables (out_variable):}

SIM_OCEAN_MODEL_REPORT - String containing the sensor type and AUV position and time that the ocean model data is being requested for, plus the ocean model data simulating the sensor outputs (i.e., temperature, salinity, \& sound speed, or u, v, \& w current components). See configuration below.

MSEAS_MODEL_TIME - String containing the model time (different from the actual time) associated with the values output in the SIM_OCEAN_MODEL_REPORT.

SIM_OCEAN_MODEL_REPORT $=$ "id=..,vname=..,stype $=\ldots, \mathrm{x}=\ldots, \mathrm{y}=\ldots$, lat $=\ldots$, lon $=\ldots$, depth $=\ldots$, time $=\ldots$, sensor_parameters"

id - Request ID

vname - Requested vehicle name

stype - Type of sensor, e.g., CTD or ADCP

$(\mathrm{x}, \mathrm{y})$ - Local coordinates of the data requested 
(lat,lon) - Global coordinates of the data requested

depth - Depth of the data requested

time - Time at which data is requested

sensor_parameters - In the case when stype=ctd, sensor_parameters will be "temperature $=. . .$, salinity $=\ldots$, soundspeed $=. . . "$ and when stype $=$ adcp it will be "u=..,v=.., $\mathrm{w}=\ldots .$.

When configuring the in_variable and out_variable fields, the order in which the variables are defined in the configuration must match the order in which they are input to and output from the Octave function/script that is being called.

\section{Other configuration parameters:}

octave_search_path - Path to where the desired Octave script resides

octave_function_name - Name of the Octave function/script to execute using the aforementioned input \& output MOOS variables

publish_time - When to publish the out_variable. NOW will publish immediately on each iteration of the Helm

For the MSEAS interface, getmseas_octave_new.m is run in Octave through pOctaverMIT. This script queries readmseaspe_octave.m and get_petim0_octave.m, which are slightly altered, Octave-compatible versions of the original MSEAS-supplied scripts for reading MSEAS NetCDF files (respectively readmseaspe.m and get_petim0.m, originally written for MATLAB). The readmseaspe_octave.m and readmseaspe.m scripts should not be changed without consulting the MSEAS group! When readmseaspe_octave.m is queried by getmseas_octave_new.m, the requested environmental data value (i.e., temperature, salinity, u current, v current, or w current) is returned for the position and time of the environmental 'sample' requested by the AUV. These environmental data are then re-packaged with the request message by getmseas_octave_new.m and published to the MOOSDB as the out_variable, SIM_OCEAN_MODEL_REPORT.

uFldOceanEnvSensor on the shoreside/topside will then re-package the report—as required to keep with the generalized sensor simulation structure of the uField toolbox - and send it to the vehicle using uField tools, as shown in Fig. A-4.

SIM_OCEANENV_REPORT $=$ "vname=..,stype =.., $\mathrm{x}=\ldots, \mathrm{y}=\ldots$, lat $=\ldots$, lon $=\ldots$, depth $=\ldots$, time $=\ldots$, 
sensor_parameters"

vname - Requested vehicle name

stype - Type of sensor, e.g., CTD or ADCP

$(\mathrm{x}, \mathrm{y})$ - Local coordinates of the data requested

(lat,lon) - Global coordinates of the data requested

depth - Depth of the data requested

time - Time at which data is requested

sensor_parameters - In the case when stype $=$ ctd, sensor_parameters will be "temperature $=\ldots$,salinity $=\ldots$, soundspeed $=. . . "$ and when stype $=$ adcp it will be $" \mathrm{u}=\ldots, \mathrm{v}=\ldots, \mathrm{w}=\ldots$.

The virtual experiments can also be configured to work without the uField tools. This is desirable in the cases where the missions being simulated are soon to be implemented on AUVs deployed in the real ocean (where uField tools cannot be used). It is also desirable in cases where multiple AUVs are being simulated on a single computer and processing power is limited (uField tools are more processing-intensive than workarounds that break out of the uField structure but achieve an identical virtual-experiment result).

To eliminate the use of the uField chain in virtual experiments, we simply bypass all of the communications that are set up in the uField community (by simply not using the 'ufld' flag described below), but retain the use of uFldOceanEnvSensor under the alias of uSimOceanEnvSensor through the creation of a new configuration plug, uSimOceanEnvSensor.plug, that is identical to that of uFldOceanEnvSensor.

\section{A.1.2 Mission Simulation}

\section{Commands:}

These commands are run from the topside and vehicle directories of the missions-* repositories to begin a virtual AUV experiment using the MSEAS ocean models.

$>$ cd /missions-lamss/topside

$>$./simulation_launch.sh mseas mseas_display warp=10

>cd /missions-lamss/vehicletype/vehiclename

(e.g., > cd /missions-lamss/auv/unicorn)

$>$./simulation_launch.sh mseas warp=10 


\section{Flags:}

mseas - Use the high-fidelity environmental fields generated by MSEAS, rather than the default databases for bathymetry and CTD simulation. Note: can be run with or without the ufld flag.

ufld - Use the MOOS-IvP uField toolbox for emulating a multinode communication environment. Replaces the default legacy network simulator iModemSim, compared to which it provides a number of advantages, most importantly the capability to simulate the network in warped time.

Note: When the ufld flag is used (thus using the uField structure for communication between the vehicles and topside/shoreside), the topside simulation must be launched before and vehicle simulations are launched. Otherwise, uField may not function properly.

\section{A.2 Topside Tools}

Two topside tools have been developed for use in virtual experiments involving MSEAS models and in real ocean deployments.

\section{A.2.1 MSEAS Display}

The MSEAS Display tool is used in conjunction with the Google Earth interface for Ocean Vehicles (GEOV) to display temperature, salinity, or currents as a dynamically updating overlay of color in Google Earth while monitoring AUVs in virtual experiments. Fig. A-5 shows a sample screen shot of this display. This display was particularly valuable for development, testing, simulation, and evaluation of the front tracking behaviors described in Chapter 5.

\section{A.2.2 CTD Display}

The CTD Display tool is used both during virtual AUV experiments in MSEAS ocean models and during real AUV deployments. This tool displays the temperature and salinity measurements and the derived sound speed and density values collected by the AUV. These four environmental properties each are plotted against sample time, 3D location, and depth, resulting in a spread of 12 plots. Fig. A-6 shows the near real-time CTD display from the Internal Wave Detection Experiment described in Chapter 4. These plots are updated each time the topside receives an acoustic packet containing the latest CTD data from an AUV, which is roughly every few minutes during real deployments and in virtual experiments. This gives the topside AUV operator 


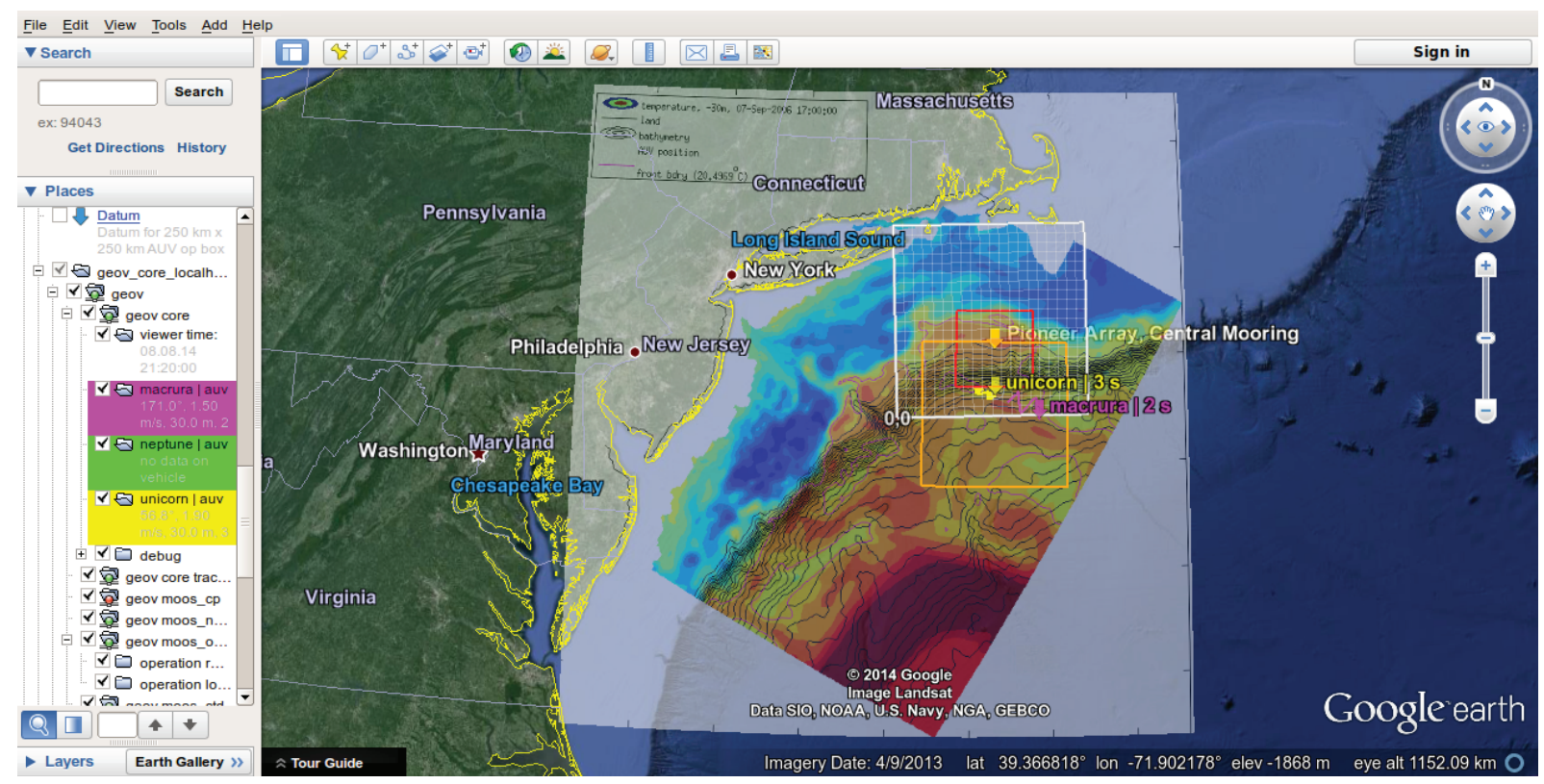

Figure A-5: The topside MSEAS display (colorful temperature overlay) running in Google Earth using the GEOV tool to visualize the paths and positions of the AUVs (yellow and magenta paths and arrows) during a virtual experiment. The MSEAS temperature overlay updates on a timer set by the user during configuration such that the dynamic environment in the MSEAS model is properly visualized through updating the overlay over time. The overlay updates are based on the current time and an AUV's current location and depth, which is especially useful when testing environmentally adaptive autonomy behaviors in virtual experiments.

a better sense of the environment an AUV is encountering in near real time, allowing for initial validation and evaluation of environmentally adaptive AUV behaviors as a mission progresses. It also allows the topside operator to predict whether irregularities in expected AUV behavior might be caused by the environment, such as poor acoustic communications at the thermocline depth or problems surfacing due to freshwater lensing. 

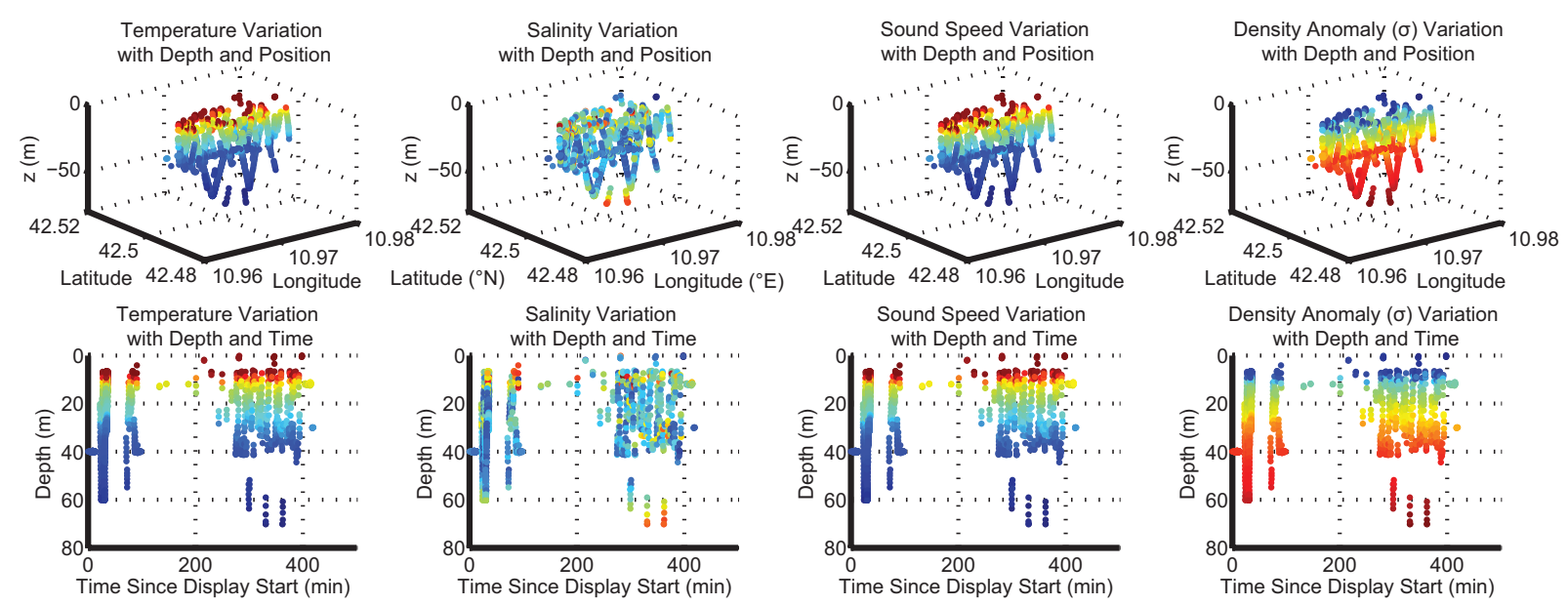

Sound Speed Variation
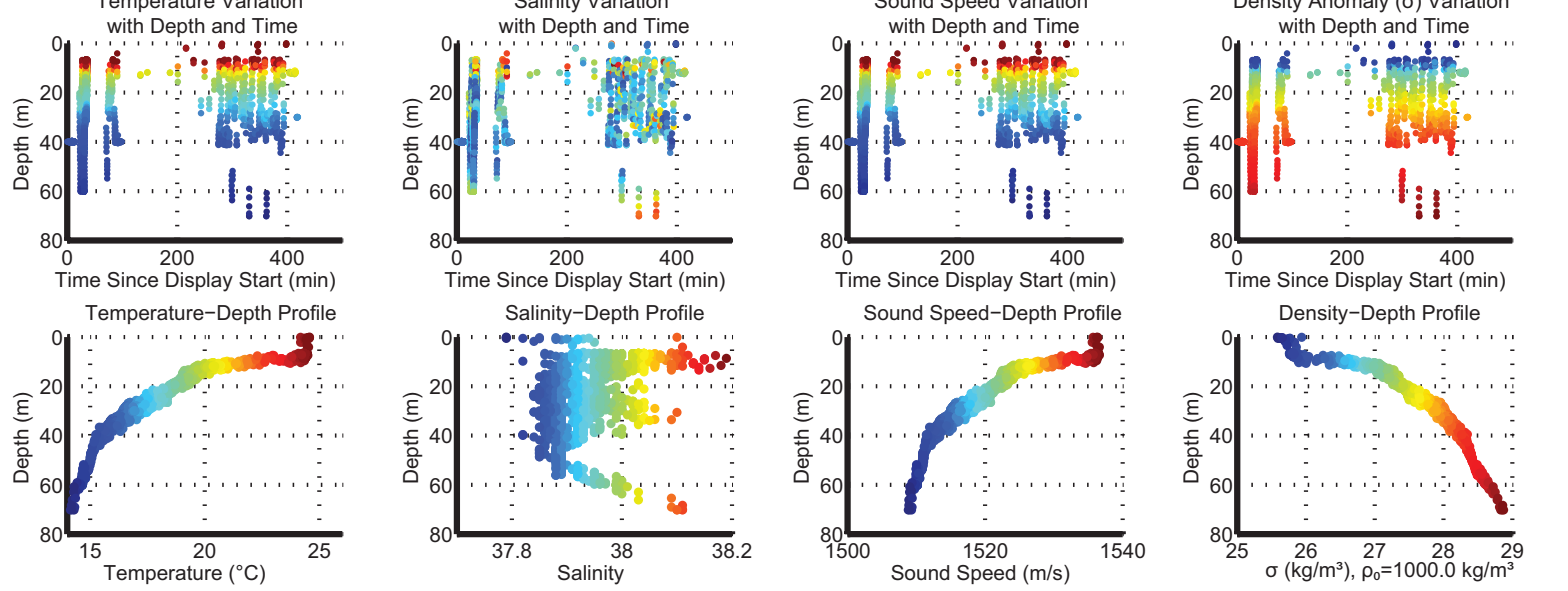

Figure A-6: The near real-time topside CTD display from the GLINT '10 Internal Wave Detection Experiment (details in Chapter 4). 


\section{Appendix B}

\section{Constructing a Distributed AUV Network for Underwater Plume-Tracking Operations}

\section{B.1 Introduction}

The underwater environment itself is hazardous to humans, as we cannot survive without air to breathe and our bodies cannot withstand the ambient pressure deep underwater, yet we could not exist without the presence of large bodies of water on our planet. The health of the oceans has a significant impact on both marine and human life. This has been observed most recently through the impact of offshore oil spill plumes and harmful algal blooms (HABs) on coastal waters. However, even in healthy ocean conditions, the ocean environment can be dangerous for humans, such as near the extreme temperatures and chemicals spewing out of hydrothermal vents into fluid clouds deep in the ocean. These features of the ocean environment create a challenge for underwater exploration and oceanographic data collection. The use of autonomous (unmanned) underwater vehicles (AUVs) in such environments is crucial to safely and efficiently completing these tasks, as they can be designed to withstand biological and chemical contaminants, high pressures, and extreme temperature variations. AUVs (especially actively-propelled ones) can also be programmed to react autonomously and adaptively to changes in their environments by controlling their own motion, unlike

This appendix is (C2012 Stephanie Petillo et al. Reprinted, with permission, from S. Petillo, H. Schmidt, and A. Balasuriya, "Constructing a Distributed AUV Network for Underwater Plume-Tracking Operations," International Journal of Distributed Sensor Networks: Special Issue on Distributed Mobile Sensor Networks for Hazardous Applications. [4] 
drifters, moored sensing arrays, or sensing buoys.

Oil spill plumes, HABs, and clouds of hydrothermal vent fluid in particular can each be viewed as a type of underwater plume (much like a cloud or plume of smoke), evolving in 3D space and over time. These plumes can range in scale from tens of meters to hundreds of kilometers in horizontal space at their neutrally buoyant depths and move with the prevailing currents, as well as spread and diffuse into the surrounding water masses $[46,68]$. Trying to track meso- and large-scale features (as plumes often are) with relatively small AUVs requires the coordinated effort of multiple AUVs, due largely to both battery life and AUV speed limitations. Willcox et al. [153] take a unique approach to this challenge in which they determine an optimal AUV survey and sampling strategy by quantifying an AUV's energy efficiency, quantifying the degree of synopticity with which an AUV can measure an ocean process, and accounting for inherent survey errors in the sampling strategy. Plume tracking also brings forth the problem of spatiotemporal aliasing of data when the plume is too large and/or moving too fast for a single AUV to collect a cohesive data set to accurately detect and track the plume edges as the plume evolves in space and time. That is, the samples taken by the AUV(s) must overlap within the plume's characteristic temporal and spatial scales to collect a synoptic data set. The importance of an ocean feature's spatial and temporal scales on feature detection and classification using AUVs is further emphasized by the work of Zhang et al. [76]. Thus, in this work we address the motivation for and challenges of constructing a network of AUVs to perform plume boundary tracking over two dimensions in space (horizontal) with time variations. We have chosen to track the boundary of a plume, rather than its center or maximum concentration, because the boundary gives a complete picture of the plume's spatial extent in the horizontal plane, where it is most likely to intersect a coastline or get entrained by currents and carried to another part of the ocean. We also present a simulated plume environment sampled by AUVs, from which we attempt to reconstruct the plume as a sum of Fourier orders as an initial estimate of the plume shape. The example of an oil leak, such as that from the Deepwater Horizon disaster in the Gulf of Mexico in 2010 [46], will be used to motivate a number of numerical assumptions in this work, though we try to keep this first-pass plume simulation as general as possible to other types of plumes as well.

In addition, it is useful to know a bit about the AUVs we are using to guide numerical values for virtual AUV experiments. For most field trials and autonomy testing, our group in the Laboratory for Autonomous Marine Sensing Systems at the Massachusetts Institute of Technology uses two Bluefin 21” AUVs (21” hull diameter, $\sim 3 \mathrm{~m}$ in length), as shown in Fig. 4-1. These vehicles demonstrate the best motion and stability control at speeds between 1 and $1.8 \mathrm{~m} / \mathrm{s}$, with navigational error of about $1 \%-5 \%$ of the distance traveled between surfacing to get a position fix via GPS. The AUVs navigate using a Leica DMC-SX Magnetic Compass 
and a Crossbow AHRS (attitude heading reference sensor). The navigational error quoted above assumes the AUV has constant DVL (Doppler velocity log) bottom-lock, has completed a compass hard iron/soft iron calibration, and has completed a compass star maneuver (for compass calibration in the water). Beyond this, the Bluefin software on the AUV also does some calibrations and math to improve the navigational accuracy to achieve the range above. To maintain reasonable stability control and navigational accuracy, the AUVs are usually commanded to travel at $1.5 \mathrm{~m} / \mathrm{s}$ (though this speed varies due to autonomous adaptation to the AUVs' situations) and surface for a GPS position fix every 30 minutes, resulting in about 50-100 $\mathrm{m}$ of navigational error. Other instrumentation currently on board consists of a conductivity-temperature (CT) sensor, a pressure sensor, and an acoustic modem with transducer, however, these vehicles could also be equipped with sensors that could measure chemical tracer concentrations or biological (Chlorophyll-a, Colored Dissolved Organic Matter, etc.) concentrations for the purposes of detecting oil, hydrothermal vent

fluid, or algal concentrations. For communicating with the AUVs (Sections B.3.3 and B.4), we make extensive (and nearly exclusive) use of an acoustic communication structure (AUV-to-AUV and AUV-to-ship/lab) that has been actively developed and refined in recent years to give virtually real-time updates (delays on the order of minutes) of scientific and navigational data (more details on this are found in the Goby project documentation $[6,7])$. Linking all of these pieces together is the autonomy system on board each AUV. This includes the Mission Oriented Operating Suite (MOOS) and the IvP Helm (IvP stands for Interval Programming), which coordinate to implement the execution of autonomy behaviors by the AUVs. These behaviors autonomously and adaptively control the heading speed and depth of the vehicle, depending on the behavior the AUV operators have chosen to run (more on this in Section B.4 and [1,2]).

\section{B.2 Spatiotemporal Aliasing Problem}

One of the most common challenges of working with AUVs to track ocean features is that of spatiotemporal aliasing. That is, when the samples taken are too far apart in space and/or time to be able to resolve the boundaries or position of a dynamic feature at a given point in space and time. This is effectively a trade-off between data coverage and data resolution. There are two extremes here (for example):

1. A single AUV can survey a small area $(\sim \mathrm{O}(1 \mathrm{~km})$, low spatial coverage $)$ with very high spatial sampling resolution $(>\mathrm{O}(1 \mathrm{sample} / \mathrm{m}))$ to resolve small-scale features in the water, such as pockets of turbulence. However, this survey would not have great enough coverage to determine the bounds of a $10 \mathrm{~km}$ wide algal bloom encompassing the sampling area. 
2. A single AUV can survey an area once over a long time period ( $\geq O(10 \mathrm{hr})$, high temporal coverage) for hydrothermal vent plumes. However, it may take so long ( $>10$ hours) to perform a spatiallycomprehensive survey, as witnessed by Jakuba et al. in [39], that the plume has advected away from its initial surveyed position during that period (poor temporal resolution) and the survey must be redone with less coverage to resolve the motion of the plume.

Somewhere in the middle of the above 'coverage vs. resolution' scenarios resides a delicate balance in which the characteristic scales of a dynamic feature (say, a plume of oil) coincide with (one half) the rate at which the feature is sampled. This is essentially a sampling of the plume at its spatial and temporal Nyquist frequencies to maximize both coverage and resolution of the plume within the data set. Thus, it is necessary to know the characteristic spatial and temporal scales of the feature of interest for more intelligent path-planning purposes (see Fig. 2-4), most likely involving multiple AUVs for tracking mesoscale features that are dominantly dynamic in two or more dimensions of space, or any feature highly dynamic in time (such that an AUV moving $\leq 2 \mathrm{~m} / \mathrm{s}$ could not keep up).

The necessity for designing a multi-AUV network to implement more intelligent and efficient mission planning is highly motivated by this aliasing problem, and relevant methods used by Zhang et al. and Willcox et al. to optimize AUV surveys and motivate the use of solo and multiple AUVs in efficient spatiotemporal ocean sampling and feature tracking will be important to take into account in implementing robust plume tracking algorithms and techniques on board AUVs [76, 153].

\section{B.3 Advantages and Challenges of an AUV Network}

\section{B.3.1 Working as a Team}

An AUV network allows for the dynamic interaction of multiple AUVs to better adapt to dynamic features in the marine environment. That is, a network of AUVs has the ability to distribute its nodes around the entire boundary of a plume and move with the plume boundary, whereas a solo AUV may be optimally placed for sampling within a plume but could not determine the horizontal spatial extent of a plume and track it simultaneously on its own. Using the estimated characteristic scales of the plume (from satellite imagery, past surveys, or physics-based calculations) in guiding the AUV autonomy behaviors (described in Section B.4), the network of AUVs can be distributed in space and time to detect and track the plume boundary and avoid aliasing the data. This desire for adaptive feature tracking also underscores the necessity for using mobile

(self-propelled) sensing platforms instead of, or in conjunction with, fixed and drifting sensing platforms (e.g. 
buoys, Argo floats) such that sampling is performed more efficiently (minimizing overlapping data) and the scientist can be certain that he/she has captured a complete data set describing the plume.

\section{B.3.2 Autonomous Coordinated Control}

The brains behind coordinating a sophisticated network of AUVs for plume tracking is the underlying autonomy system that must run on board each AUV. An autonomy system, such as that described in Section B.4, allows an AUV to adapt to its environment in near real time, without human intervention. A few of the minimum requirements of using and interacting with a robust autonomy system are inter-AUV (acoustic) communications, support for adaptive autonomy behaviors (supplied by the user) to be executed by the AUVs, and an intelligent (autonomous) means of deciding which behaviors have priority during a given mission. We propose a tiered mission planning structure for this system in which the large-scale, overall mission drives the initial formation of the AUVs (assigning each an initial position), and then allows each AUV to use individual autonomy behaviors to follow the plume edge in its local vicinity. After a period of time, the local data collected by all AUVs is then exchanged across the network to update the plume model and, subsequently, the large-scale mission of the AUVs. From here the overall mission, to local missions, to data collection, exchange, and reprocessing loop continues for as long as required by the scientist/user.

\section{B.3.3 Acoustic Communication}

One of the primary challenges using multiple AUVs simultaneously in the underwater environment is that of communication. Radio-frequency (RF) waves are quickly attenuated in the water within a few meters of the surface, leaving acoustics as the primary method of real-time underwater communication. Until now, there have been few (if any) options for intelligent multi-AUV (>2 AUVs) acoustic communication schemes, though the Goby underwater communication and autonomy project (version 2.0) strives to remedy the need for coordinated message queuing and passing between multiple (and potentially an unknown total number of) AUVs $[6,7]$. This will allow each AUV to discover and communicate with neighboring AUVs and share data and knowledge with the sensing platforms in its underwater network. As this part of version 2.0 of the Goby project is still in development, it is currently undergoing initial field testing and will hopefully come into use in the next year.

It is important to note, however, that plumes are often meso-scale features or larger, and AUV-to-AUV and AUV-to-ship/lab acoustic communication (at least in the public domain and on power-limited AUVs) is only possible up to a range of about $10 \mathrm{~km}$. Our group at MIT has found that our equipment is usually 
limited to about $2 \mathrm{~km}$ of acoustic communication range in the coastal ocean and lake environments we have performed most experiments in recently. Our Bluefin 21" AUVs and lab setup, which are each equipped with a WHOI Micro-modem and model WH-BT-2 $28 \mathrm{kHz}$ transducer, transmit data in the frequency band of $23-27 \mathrm{kHz}$, centered around $25 \mathrm{kHz}$ [8]. There are two realistic solutions to the acoustic communication range restriction we experience. The first and more complex solution is to implement a multi-hop acoustic communication scheme in which data from one AUV is passed down through a chain of AUVs to its destination. This is time consuming due to the nature of sending and listening for transmitted data packets one at a time between communicating AUVs. Given that AUVs will often be hundreds of meters apart or more and sound speed propagation is about $1500 \mathrm{~m} / \mathrm{s}$ in the ocean, data packets take an observable amount of time to transmit through the water $(\mathrm{O}(1 \mathrm{sec}))$. This method would also require extensive research into data routing on dynamic and time-scheduled messaging networks. The second and more immediately feasible (potentially more reliable) solution would be to restrict communication of large environmental data sets to RF or satellite methods while an AUV is on the surface and utilize a delay tolerant network rescheduling scheme. Although this method removes much of the real-time underwater data passing between AUVs (with the exception of basic position updates of nearby AUVs for avoiding collisions), it would take a large burden off of the acoustic channel and still allow each AUV to be re-directed based on the most current overall picture of the plume while still performing solo autonomous and adaptive plume boundary tracking in its local vicinity in real time. Periodic surface communication would work best in the case that the AUVs can surface with great enough frequency (within the characteristic time scale of the plume) to be re-directed to a more optimal sampling position, but with low enough frequency that the plume tracking mission is not significantly disrupted by the AUV taking the time to come to the surface more often.

\section{B.3.4 Data Fusion}

The fusion of data both from multiple sensors on a single AUV and all sensors across all networked AUVs is crucial to the success of coherently adapting a fleet of AUVs to track an ocean feature and collect a synoptic data set. When fusing data from a single vehicle, the largest concerns are keeping all data accurately timeand position-stamped. Across multiple AUVs, the data must also be quality-checked for corruption during transmission after passing it from one vehicle to the next. It is proposed that, on board each AUV, the computer must mesh the data sets from all AUVs into a single data set, sorted over the times and positions at which each data point was taken, for each variable (i.e., temperature, salinity, etc.). Upon processing of these data on board (as on-board processing is the only way to adapt to a dynamic environment in real time), 
for each variable, probability weighting functions over time and space must be applied to each data point based on the characteristic spatiotemporal scales of that variable. We prefer to use a basic Gaussian-shaped weighting function for this task. This will associate, say, all temperature readings taken in the last few minutes and within a radius of a kilometer horizontally (assuming the AUV can resolve its position with even better accuracy), but will ignore any temperature readings that fall outside of these ranges as independent from those inside. This essentially creates an overlap of data within a radius of one standard deviation about the sample point, as sketched in Fig. 2-2, that can be used to prevent insufficient sampling in a data set. This data fusion method could be implemented using an SQLite (or similar) database on each AUV to compound and sort all of the environmental data from all AUVs, which may then be processed in a mathematics program such as MATLAB or Octave, or by a simple $\mathrm{C}++_{+}$parser with algorithms utilizing $\mathrm{C}++$ vector math libraries. This is similar to creating an evidence grid of the AUVs' environmental data [13]. The resulting ocean environment reconstructed through data fusion with weighting can guide the mission planning for a fleet of AUVs tasked to track a plume. The AUVs can survey an area with high enough resolution to find the boundary of the plume, approximate the plume shape (see Section B.5) with higher weighting near the actual sample points, and revise their coordinated survey strategy based on this new estimate of the plume boundary position.

\section{B.4 Adaptive Behavior Implementation}

When conducting field experiments with AUVs (usually only 1 or 2 ) in the water, our group at MIT runs the Mission Oriented Operating Suite (MOOS) as the underlying autonomy system on board the AUVs and on our topside mission-command computer. MOOS provides a publish-subscribe architecture that essentially deals with information sharing between autonomy processes and behaviors on board each AUV, as well as through the water between the AUVs and the topside computer [1]. To add some intelligence to the system, the IvP Helm (IvP stands for Interval Programming) is used in conjunction with MOOS to implement the use of autonomy behaviors (e.g., vertical yo-yos, trail-an-AUV, horizontal racetracks, safety behaviors) on the AUVs, optimizing over the vehicle's speed, heading, and depth $[1,2]$. The acoustic communications are handled through the Goby (stable version 1.0) autonomy software on all platforms, where it schedules the transmissions of each node (AUVs, communication buoys, topside operator, etc.) in the network [6,7]. Goby encodes data on one node, initializes the data transmission through the acoustic channel, and then decodes the data when they are received on another node. All of these pieces to our autonomy architecture allow our AUVs to adapt their motion based on sensor readings, without a human in the loop. This allows for ocean

feature detection and tracking by AUVs to occur both autonomously and adaptively, as demonstrated in the 
following examples.

\section{B.4.1 Thermocline Tracking as a Proof-of-Concept}

The aforementioned autonomy system has been put to the test in performing autonomous, adaptive thermocline tracking in the Tyrrhenian Sea (Italy) and Lake Champlain (Vermont, U.S.A.). As described in [3], a simple thermocline tracking algorithm, which also accounts for the characteristic scales of the thermocline, has been developed and tested over the past few years using single AUVs of varying manufacture. Fig. B-1 is a conceptual sketch of the adaptive thermocline tacking process, while more detail can be found in [3]. The idea here is that the thermocline, which is a feature only qualitatively defined in most oceanographic literature, must be quantitatively defined using actual data in real time for more efficient and adaptive oceanographic sampling. Here it is assumed that the thermocline is relatively homogeneous in horizontal space within the AUV's operational region (for our vehicles, usually about $25 \mathrm{~km}^{2}$ or less). That is, given an AUV's temperature measurements through the water column, on-board processing of the temperature data is accomplished spatially in $1 \mathrm{D}$ by binning the temperatures by depth ranges smaller than the characteristic (vertical) length scale of the thermocline in the experimental area $(\mathrm{O}(10 \mathrm{~m})$ in shallow water) and using finite differences to determine the region of greatest change in temperature over change in depth. The characteristic time scale of shallow water thermoclines (in the regions this algorithm has been tested) was determined by observation during our field trials to be $\mathrm{O}(1 \mathrm{hr})$. Thus, temperature measurements were averaged over windows of 30 minutes to smooth out small local variations and spurious data points. Once the thermocline region has been determined by the AUV, the AUV will autonomously adapt its depth range to stay within the current boundaries of the thermocline and continue to collect a synoptic data set through the thermocline without expending extra energy to dive unnecessarily deeper or shallower.

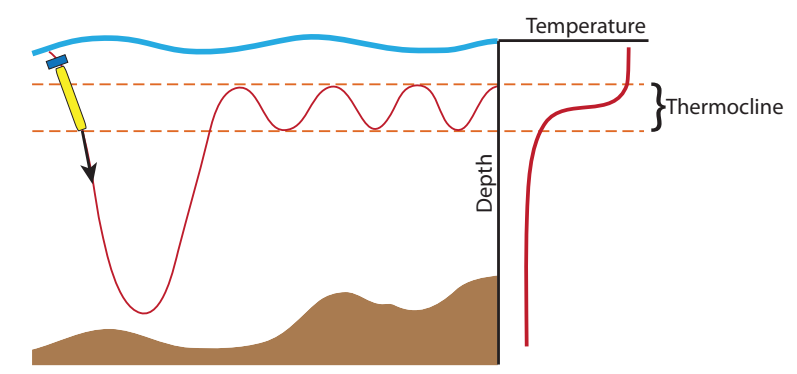

Figure B-1: A conceptual sketch of an AUV performing thermocline tracking. The AUV completes a dive from the surface to as deep as allowable, collecting temperature data. The depth range of maximum temperature change per unit depth is determined as the thermocline region. The calculated upper and lower bounds of the thermocline region are then used to bound the vertical yo-yos of the AUV, essentially tracking the thermocline region. Used with permission from [3]. 
The successful field testing of this thermocline tracking process serves as a proof-of-concept for the feasibility of performing adaptive, autonomous feature tracking with an AUV, guided by the feature's spatial scale in 1D (vertically) and temporal scale to drive intelligent and efficient data collection. Thermocline tracking provides a solid first stepping stone into the field of multi-dimensional oceanographic feature tracking, from which we can move on to implementing applications with more complex features (dynamic in 2D or 3D space, and time) such as underwater plumes.

\section{B.4.2 Plume Tracking}

Plume detection and tracking using AUVs has come to the forefront of the oceanographic research community in recent years through the impacts of HABs and oil spills on coastal populations and the intrigue of studying the alien environment in the vicinity of hydrothermal vents. Smith et al. uses a regional ocean model to predict the advection of a patch of water representing a HAB off the California coast, which is tagged by an actual Lagrangian drifter to passively mark and track the centroid of the imaginary HAB. AUVs (gliders) are then deployed to arrive at waypoints on the approximate boundary of the HAB when the HAB is predicted to reach that point. The calculated arrival paths of the AUVs are based on the plume boundary predictions from a regional ocean model, and the waypoints of the gliders are updated every few hours based on the previous dive's data and the model's predictions of the future boundary location of the advecting the patch of water [41]. Similarly, Das et al. uses satellite and high-frequency (HF) Radar data sets to determine the location of high-concentration HAB patches and targets these 'hotspots' using AUV (glider) path planning algorithms guided by the paths of the drifter tags for finer resolution sampling [42]. In a second paper, Das et al. expand this $\mathrm{HAB}$ tracking method further to perform Lagrangian observation studies in which the AUVs' (gliders') survey paths are pre-calculated to survey an advecting patch of water in its Lagrangian frame of reference to maintain sufficient spatial and temporal data resolution [50].

The difference between the aforementioned literature and the implementation methods in this work lie in the ability of the propelled AUVs we propose to use to exhibit much better navigation control, faster speeds, limited but sufficient acoustic communication while underwater, entirely on-board data processing, and real-time feedback and reaction to sensed changes in the ocean environment without a human in the loop (no path-planning algorithms or predetermined paths/waypoints fed to the AUV by scientists), which makes the AUVs truly autonomous and adaptive. This is, of course, at the cost of the battery duration of the AUVs, which must be recharged much more frequently. Since complex dynamic ocean models are often very large, it is not realistic to run them on board AUVs that must be fully autonomous. Satellite and HF Radar 
images are only useful for detecting plumes with surface expressions, eliminating their usefulness in detection of neutrally buoyant plumes below the top $10 \mathrm{~m}$ of water. Thus, we seek to develop a method of plume tracking that can rely solely on the environmental data collected over space and time by the AUVs. The only caveat here is the assumption that a single initial large-scale survey has already been done by an AUV or other sensing platform (or a recently updated regional ocean model has been run) in the region encompassing the plume such that an approximate plume boundary location at the plume's neutrally buoyant depth is known at the time of AUV deployment. The details of obtaining this initial plume boundary location are beyond the scope of this appendix, but are addressed in Chapter 6.

As mentioned in Section B.3.1, it is useful to approach plume tracking by knowing something about the general dynamics and characteristic scales of the plume, as well as any information about its source (for oil leaks or hydrothermal vent sites) or ocean conditions necessary for occurrence (for HABs), and what data values from various sensors might signal that a measurement was taken inside a plume. As mentioned above, since there are many approaches to first detecting a plume that are beyond the scope of this appendix, we will assume here that the initial $2 \mathrm{D}$ boundary of the plume in the horizontal plane has been detected or approximated via satellite imagery, recent oceanographic surveys, or the physics of the region of interest before any AUVs are deployed to track the plume. We will start by concerning ourselves with the horizontal extent of the plume at its neutrally buoyant depth, over a time span shorter than the plume's characteristic time scale (over which the plume boundary displays only minor variations in position). From here we can sample the plume boundary (defined by a threshold chemical or biological concentration value during field experiments) with varying numbers of AUVs and estimate the plume shape as a sum of Fourier orders.

With an estimation of the location of a plume boundary at a given depth, multiple AUVs (preferably enough to maintain slightly overlapping one-standard-deviation spatial scale range circles along the plume boundary within the plume's characteristic time scale, similar to the range rings in Fig. 2-2) can be deployed within the plume, and an algorithm can be used to assign each AUV a starting position near the estimated plume boundary with approximate equal spacing azimuthally between AUVs about the estimated plume center point. This initial AUV spacing can be written into an IvP Helm 'equal azimuth angle' autonomy behavior that would attempt to maintain equal azimuthal spacing of the AUVs, even as they progress along the plume boundary and the boundary shifts position, adjusting the speed of each AUV to compensate if any one gets too far ahead or falls behind. A second tier of autonomy control will govern the reactions of each AUV to its local environment with a 'plume boundary tracking' behavior. This behavior will have a threshold concentration value set for whatever tracer is used to signify levels of chemicals or biological productivity are 
indicative of the plume of interest. The plume boundary tracking behavior will direct the AUV to zigzag horizontally back and forth across the position of this threshold (as it travels azimuthally around the plume center) to maintain an up-to-date position of the local plume boundary. Finally, on a time interval sufficiently small (less than the characteristic time scale of the plume) to average these data over time from each vehicle, each AUV will share its collected plume boundary position data with the other AUVs in the vicinity via acoustic (or RF or satellite) communication, and each vehicle will sort and process the collective data to determine the most current plume boundary position by estimating it as a sum of Fourier orders. Each AUV can then determine if it needs to adjust its speed and big-picture position about the plume edge using the equal azimuth angle behavior. Not only will this method of plume tracking capture the shorter/smaller-scale variations of the plume form one time interval to the next, but also create a continuously evolving track of plume evolution in space and time for a given depth.

With further development to track a plume over longer time scales, we will be able to detect the radial expansion rate of the plume boundary (if any) and its development due to advection, diffusion, and/or bological processes, and thus forecast its motion to improve forward-looking mission planning. The best way to develop this plume tracking process is through simulation, as described in Section B.5. Once the simulation is complete, we will be able to initialize implementation of autonomous and adaptive plume tracking with our autonomy architecture by simulating AUVs, (acoustic) communication, and data fusion as described in Section B.3 until the plume tracking algorithms and their supporting autonomy behaviors are robust enough for field testing.

\section{B.5 Plume Simulation Environment}

Towards the goal of developing plume-following strategies for AUVs, we must first get a sense of the characteristics of a plume and what the best method is in distributing AUVs about the plume. This requires examining the results and errors associated with reconstructing the shape of a simulated plume from simulated AUV sample points along the plume's edge. Instead of diving into incorporating a more robust or dynamic plume model developed by an outside group, we choose to simulate a very simple plume boundary in horizontal space using Fourier orders (a rough 2D plume approximation) such that we could exactly reconstruct the original plume (again by using Fourier orders) under ideal (though very unrealistic) conditions. This gives us validation that our plume reconstruction algorithms were derived correctly. Though we introduce a few sources of plume reconstruction error in the plume simulation and reconstruction process described in this and subsequent sections, we expect to incorporate a more realistic, already developed plume model in the near 
future such that we are not setting up a situation in which our simulation is doomed to (mostly) succeed. As this is a first-pass simulation experiment, the effects of advection, dispersion, diffusion, holes in the plume shape, multiple plume sources, algal life cycle dynamics, and other complexities that may influence a plume's development over time are beyond the scope of this work. To test our algorithms and experimental setup over a simulated characteristic plume time scale, we expand the plume in the horizontal plane over a short period of time, sample the plume boundary with varying numbers of AUVs (approximating navigation errors), and then reconstruct the plume from these time-varying sample points. This process is described below.

\section{B.5.1 Modeling a Plume}

A rough estimate of a plume boundary in the horizontal plane is achieved using Fourier orders of the form

$$
\Phi_{M_{h i}}=\Sigma_{m=0}^{M_{h i}}\left[A_{m} * \cos \left(m \theta+\phi_{m}\right)\right]+R,
$$

where $M_{h i}$ is the highest Fourier order of the series (here we will solve for a plume of $M_{h i}=20$ orders by estimating it with up to 8 Fourier orders from AUV sample points), $A_{m}$ is the radial amplitude perturbation of the plume boundary for the $m^{t h}$ order, $\phi_{m}$ is the phase shift of the $m^{t h}$ order, and $R$ is the unperturbed radius of the plume. The angles, $\theta$, are in the range $[0,2 \pi)$ rad about the center of the plume, and $\Phi_{M_{h i}}$ is the radial distance to the edge of the plume from the center at each angle, $\theta$, for a maximum Fourier order, $M_{h i}$. Generating coefficients $A$ and $\phi$ at random for each $m$ results in the progression of plume development

shown in Fig. B-2, leading to the overall 'actual' plume in Fig. B-3. We have bounded $A_{m}$ to $\pm \frac{R}{2 m}$, placing the most energy in the lower orders to somewhat realistically represent the amplitude variations of the plume and minimize sharp radial inversions in the boundary shape.

Although it is possible to solve for a very large number of Fourier orders (given enough AUVs over time), this is not computationally efficient and (as seen in Section B.5.4) has diminishing returns. Using a sum of many Fourier orders, however, is the most realistic approach (in this simulation) to adding complexity to the simulated plume shape. Time variation (within the characteristic time scale of the simulated plume) is also incorporated into this model, providing more total sample points per AUV (Section B.5.2). Over time scales greater than the characteristic time scale of the simulated plume, it is also possible to simulate the development of the plume through turbulent and diffusive processes, as well as represent the effect of dominant currents and algae life cycles on the plume shape. Though the effects of long term time variation have yet to be incorporated into the plume simulation, we describe a means of simulating, detecting, and forecasting basic longer-time-scale radial variations in Section B.6. 

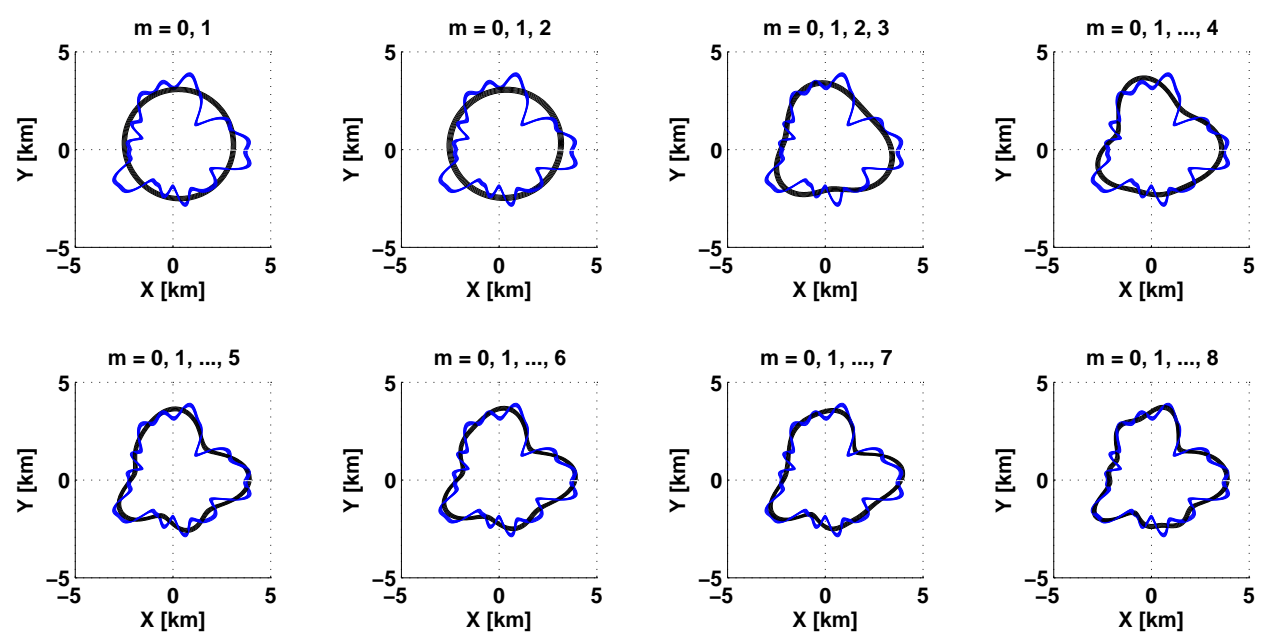

Figure B-2: A progression of simulated plume shapes (black) of $R=5 \mathrm{~km}$, building up to $M_{h i}=20$ (blue).

\section{B.5.2 Sampling a Plume}

First, it is important to backwards-engineer the simulated plume as follows to be sure that the AUV-sampledplume boundary reconstruction algorithms are correct. In a perfect world (with obviously unrealistic assumptions) in which a plume is exactly delineated by a finite sum of Fourier orders and AUVs are evenly spaced around the center of this sharply defined plume at a radius that is on the exact boundary (no navigational error), theory suggests that $2\left(M_{h i}+1\right)$ AUVs are necessary to exactly solve Equation B.1 for its $2\left(M_{h i}+1\right)$ unknowns (here we assume that we can approximate $R$ as the average of all AUV distances radially from the plume center, $\left.\Phi_{a v g}\right)$. However, since the $0^{\text {th }}$ order is of constant radius, we incorporate $\cos \left(\phi_{0}\right)$ into $A_{0}$ and say $\phi_{0}=0 \mathrm{rad}$, reducing the number of unknowns (and AUVs) to $2 M_{h i}+1$. Noise may be added to the angular and radial positions of the AUVs to simulate navigation error and the imperfection in trying to coordinate multiple AUVs spaced at exact angles about a circle, on the exact radius of the plume. Further error will arise from the use of a finite number of AUVs and the necessity of approximating a high order plume with an often relatively low number of Fourier orders calculated from AUV sample points. Since plumes in the ocean and in more robust plume models cannot be fully characterized in closed form as a sum of Fourier orders, error will inherently be added to the AUVs' Fourier order plume reconstruction when real data or data from a more robust model are used.

Time steps (within the characteristic time scale of the plume boundary position) may be added to increase the number of sample points available, giving $N_{\text {samples }}=N_{\text {timesteps }} * N_{A U V s}$, and to increase the maximum 


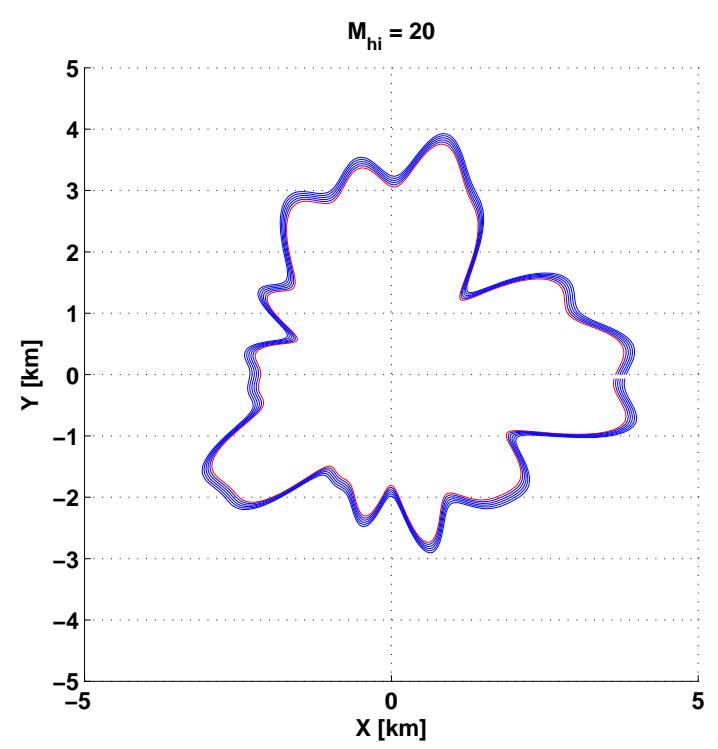

Figure B-3: The 'actual' plume of $M_{h i}=20(R=5 \mathrm{~km})$.

number of Fourier orders, $M_{A U V, \max }$, that can be used to solve for the plume boundary shape. In this implementation, we applied a bounded, random, linear rate of (positive) radial expansion to the amplitude of each Fourier order in the 'actual' plume, examining time steps of 2 minutes over a sufficiently small period of 10 minutes for a plume expanding radially at a rate of up to $0.5 \mathrm{~m} / \mathrm{s}$. In real-world applications, this expansion rate is based upon the vertical flow rate from the plume's source (if present; counteracted somewhat by buoyancy changes with depth) and horizontal spreading (via advection) and diffusion of the plume at the sampled depth [68]. If dealing with a HAB, the life cycle of the algae must also be considered.

\section{B.5.3 Reconstructing a Plume from AUV Sample Points}

Given $N_{A U V}$ AUVs located about the plume boundary at an instant in time, at radii, $\Phi_{A U V}$, at known angles, $\theta_{A U V}$, a fast Fourier transform algorithm, $f f(\bullet)$, is applied to these data to determine the unknown coefficients of the plume with Fourier orders $M \leq\left\lfloor\left(N_{A U V}-1\right) / 2\right\rfloor$. The following algorithms are then used to extract out the coefficients.

$$
\begin{gathered}
R \approx \Phi_{a v g}=\frac{\Sigma_{\theta_{A U V}} \Phi_{A U V}}{N_{A U V}} \\
A_{A U V, m=0}=\frac{1}{2} * \frac{\left|f f t\left(\left.\Phi_{A U V, m=0}\right|_{\theta_{A U V}}\right)\right|}{N_{A U V} / 2}-\Phi_{a v g}
\end{gathered}
$$




$$
\begin{gathered}
A_{A U V, m=1: M}=\frac{\left|f f t\left(\left.\Phi_{A U V, m=1: M}\right|_{\theta_{A U V}}\right)\right|}{N_{A U V} / 2} \\
\phi_{A U V, m=0: M}=\text { angle }\left[f f t\left(\left.\Phi_{A U V, m=0: M}\right|_{\theta_{A U V}}\right)\right]
\end{gathered}
$$

From coefficients $A_{A U V, m}$ and $\phi_{A U V, m}$, we reconstruct the AUV-derived estimation of the plume boundary, $\Phi_{A U V, M}$, as we constructed it in Equation B.1:

$$
\Phi_{A U V, M}=\Sigma_{m=0}^{M}\left[A_{A U V, m} * \cos \left(m \theta_{A U V}+\phi_{A U V, m}\right)\right]+\Phi_{a v g} .
$$

The reconstructed plume should match the original $M_{h i}$-order plume exactly (except for numerical roundoff error) when all of the following criteria are met:

- $M_{h i} \leq M_{A U V, \max }=\left\lfloor\left(N_{A U V}-1\right) / 2\right\rfloor$, that is, the maximum Fourier order used to construct the original plume is less than or equal to the maximum Fourier order used to reconstruct it from AUV data (in reality $M_{h i}=\infty$, so this could never be achieved),

- $\Phi_{\text {avg }}=R$,

- there is no AUV navigation error,

- there is no time variation,

- all AUVs are evenly spaced about the plume center and exactly on the boundary, and

- there is instantaneous all-to-all communication of data.

Obviously, some error is introduced when any one of these criteria is not met. If time steps are used to increase the number of sample points, thus increasing $M_{A U V, \max }, N_{A U V}$ should be replaced by $N_{\text {samples }}$ in all equations in this section (B.5.3), and the spacing of the clustered AUV samples must be interpolated to equal angular spacing about the plume edge to perform the fast Fourier transform (we have used a cubic interpolation function).

\section{B.5.4 Results}

A set of plume estimates of Fourier orders 1 through 8 are plotted in Fig. B-4 in contrast to the 'actual' time-varying plume. These plots also show the non-interpolated (with navigation error) and interpolated 
AUV positions. The 'actual' plume was chosen to have $M_{h i}=20$ to keep the high-frequency variations on boundary radius to a minimum while maintaining more higher-order variation that a reasonable number of AUVs $(<10)$ can exactly resolve. Other numerical assumptions had to be made for the sake of simulation testing and evaluation based on the Bluefin 21" AUVs that our lab group operates and the approximate area and expansion rate of a meso-scale plume (similar to that of the Deepwater Horizon disaster in the Gulf of Mexico in 2010 [46]). Specifically, we take $R=5 \mathrm{~km}$, AUV navigation error $=100 \mathrm{~m}$, and time steps $=0,2,4, \ldots, 10$ min within the characteristic time scale of plume evolution.
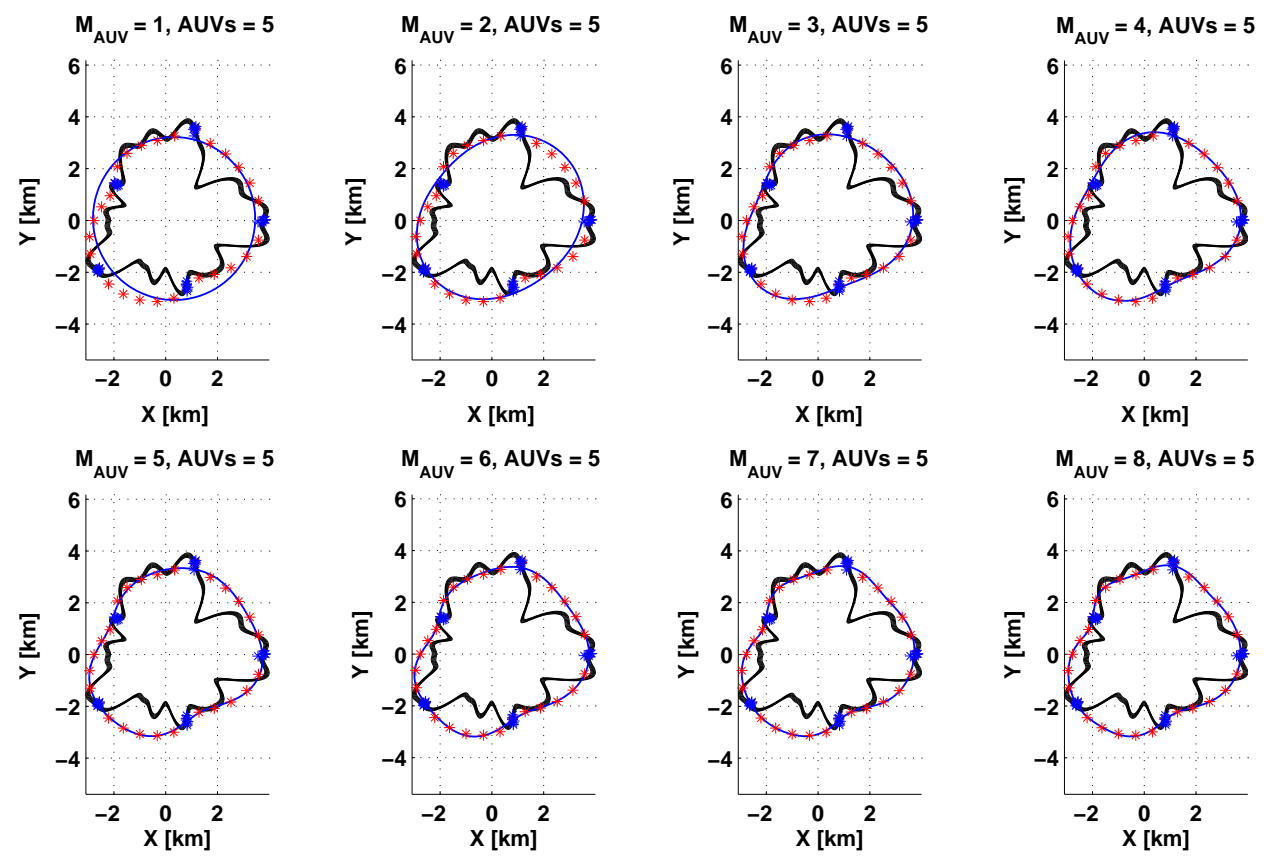

Figure B-4: Plume estimates (blue line) of $M_{A U V}=1,2, \ldots, 8$ for 'actual' plume of $M_{h i}=20$ (black lines, time-varying), $R=5 \mathrm{~km}$, navigation error $=100 \mathrm{~m}$, and time steps $=0,2,4, \ldots, 10 \mathrm{~min}$. For the 5 AUVs, the non-interpolated (blue stars) and interpolated (cubic interpolation, red stars) AUV sample points are also shown for reference.

A set of Monte Carlo simulations was used to quantify the overall mean percent error in the model based on the number of Fourier orders solved for, varying the number of AUVs while keeping the time steps consistent over all trials. This is accomplished by comparing the boundary of the estimated plume to the time-averaged boundary of the actual plume as follows:

$$
\% \text { Error }_{\text {plume }}=\frac{\left|\Phi_{\text {estimated }}-\Phi_{\text {actual }, \text { time-avg }}\right|}{\Phi_{\text {actual }, \text { time-avg }}} .
$$

These results are shown in Fig. B-5 for each set of Fourier orders, with $M_{A U V, \max }$ determined by 
$N_{\text {samples. }}$

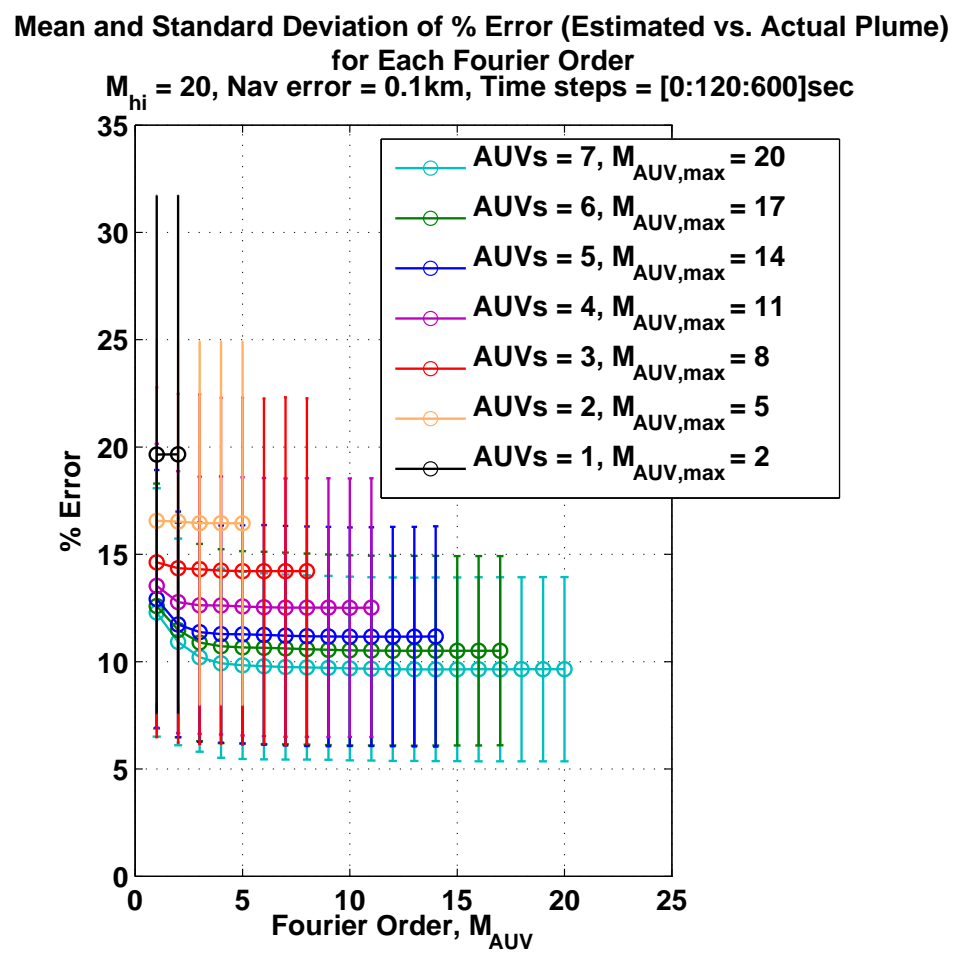

Figure B-5: Percent error in plume radius between the estimated and actual (time averaged) plumes, averaged over 500 trials. Mean values are shown for each maximum Fourier order, with error bars showing \pm 1 standard deviation.

It is interesting to note that, for a fixed number of AUVs, the general trend appears to be an exponential decrease in error as a larger number of Fourier orders is solved for. However, upon closer examination of the error values, the order of lowest error is approximately $M_{A U V \text {,min_error }}=2 N_{A U V}$. This result will help minimize the error while reasonably limiting the amount of data processing necessary to estimate the plume boundary. Alternately, for a given Fourier order $M_{A U V}$, as the number of AUVs increases, the percent error decreases, as is expected.

\section{B.6 Forecasting Long Term Variations}

Having simulated and analyzed a plume over a short time span, we will now explore expanding the plume simulation to longer time spans to enable plume shape forecasting. There are two formulations here for the basic time expansion approximation. We may either assume that the plume expands linearly in time in the radial direction, with a constant coefficient of expansion, $\frac{d \Phi}{d t}$ (Equation B.8), or that both the amplitude and phase coefficients change linearly in time, with constant coefficients $\frac{d A}{d t}$ and $\frac{d \phi}{d t}$ (Equation B.9). These are 
the most simplistic cases, which may be built upon in the future into nonlinear coefficients to account for further complexities from real ocean dynamics.

$$
\begin{aligned}
\Phi_{M_{h i}}(\theta, t) & =\Sigma_{m=0}^{M_{h i}}\left[A_{m} * \cos \left(m \theta+\phi_{m}\right)\right] \\
& +\frac{d \Phi}{d t} *\left(t-t_{0}\right)+R \\
\Phi_{M_{h i}}(\theta, t) & =\Sigma_{m=0}^{M_{h i}}\left[\left(A_{m}+\frac{d A}{d t} *\left(t-t_{0}\right)\right)\right. \\
& \left.* \cos \left(m \theta+\left(\phi_{m}+\frac{d \phi}{d t} *\left(t-t_{0}\right)\right)\right)\right] \\
& +R
\end{aligned}
$$

Assuming one of the above plume formulations and sampling it with AUVs over a number of large time steps, we can determine the differences in overall plume shape from one point in time to the next and back out the constant coefficients from there. If the formulation in Equation B.8 is assumed, we may simply find the mean difference (over all $\theta$ ) in radius between the estimated plumes at times $t_{0}$ and $t_{1}$, as shown in Equation B.10.

$$
\frac{d \Phi}{d t} \approx \operatorname{mean}\left[\frac{\Phi_{A U V, M}\left(\theta, t_{1}\right)-\Phi_{A U V, M}\left(\theta, t_{0}\right)}{t_{1}-t_{0}}\right]
$$

Solving for the Equation B.9 formulation coefficients is more complex. Given estimated plumes from AUVs at times $t_{0}$ and $t_{1}$ sufficiently far apart in time, we must maximize the correlation between $\Phi_{A U V, M}\left(\theta, t_{1}\right)$ and $\Phi_{A U V, M}\left(\theta, t_{0}\right)$ over radius and azimuth angle. The tool for this will be a matched filter applied to $\Phi_{A U V, M}\left(\theta, t_{1}\right)$ and $\Phi_{A U V, M}\left(\theta, t_{0}\right)$, allowing us to back out the constant coefficients once we determine the phase and amplitude changes between $t_{0}$ and $t_{1}$. Repeating either of the above processes over multiple time steps will further improve the accuracy of the coefficients.

Once we solve for the constant coefficients using either of the above methods, a forecast can be made for the plume shape by simply applying the linear changes to the estimated plume shape at the last known time slice, and projecting it forward to the next time step(s). As with any forecasting, however, the accuracy of the forecast decreases with time steps further into the future. A weighting function (potentially the right side of a Gaussian) should be included with the forecast to account for this. 


\section{B.7 Looking Ahead}

It is important to take what we have learned from this exercise and apply it to a more robust plume simulation, such as a theory- and data-derived dynamic plume model, as well as to prepare for taking this application into the field. Following the first iteration of this plume simulation, the next step is to use the plume estimated by the AUVs over progressive time steps to estimate the linear time perturbation coefficients of each Fourier order and use these coefficients for future prediction.

Jumping ahead to prepare for realistic implementation of plume tracking in the field, we plan to use our IvP Helm and Goby autonomy to move the AUVs along the actual plume boundary (in 'follow the leader' fashion) as described in Section B.4.2, autonomously adapting their tracks to their real-time measurements by zigzagging across the boundary, and keeping their angular spacing relatively constant. As AUVs travel along the boundary azimuthally, all at the same speed, the radial excursions in the boundary may cause the azimuthal spacing of adjacent AUVs to degrade. To counter this effect, we will employ autonomy behaviors to change speed and maintain azimuthal distribution when a significant degradation in spacing is detected. This will first be implemented in virtual experiments to work out any bugs before taking it into the field with the AUVs.

Other features to add to the plume simulation will account for the effects of advection by currents and turbulent diffusion. A good estimation for diffusion, used widely in the underwater community, is Fick's Law [154], and examples of current effects can be found in [46] and [39]. These effects may be best incorporated into the simulation as time varying coefficients similar to those in Section B.6, only nonlinear in time and space. Another option would be to take advantage of a commercial computational fluids simulator to simulate these effects. It will also be important to account for the direction of motion of a plume, as this may or may not cause the leading edge of the plume to be more distinct than the trailing edge. Again, however, we do not want to reinvent the wheel and may prefer to research and take advantage of already-existing plume models and data that account for some of these effects with greater detail and accuracy than achievable by the above method. In the case of the evolution of $\mathrm{HABs}$, we must also account for life cycle evolution of the algae, and testing with historical data of algal bloom evolution would be useful here [42].

Finally, it is important to gain a knowledge of how each source of error (i.e., navigation error, higher modes and sharper inversions in plume shape, overall plume radius, cubic interpolation of AUV spacing about the plume, etc.) affects the overall error in the estimated plume boundary. Such an error-review will require a wide range of tests, changing only one variable at a time. The cubic interpolation of AUV position alone will be evaluated against other interpolation techniques, such as the Lomb-Scargle method [155], to 
minimize errors.

\section{B.8 Conclusion}

This appendix provides a conceptual outline of the requirements for implementing adaptive, autonomous plume tracking using a network of AUVs, including a first-pass simulation of detecting and reconstructing plume shapes solely from AUV sample points, with the example of a plume of oil originating from the sea floor. Using a sum of $M_{h i}$ Fourier orders to represent a plume shape at its neutrally buoyant depth, we added noise in the AUV positions to represent navigation error. We also incorporated linear radial expansion of the plume over time to simulate plume spreading due to the continuous influx of oil. Reconstruction of the plume from the time-varying AUV samples was seen to result in errors in the estimated versus original plume shapes ranging from 9-20\% (for 1 through 7 AUVs, $M_{h i}=20, R=5 \mathrm{~km}$, navigation error $=100 \mathrm{~m}$, and time steps $=0,2,4, \ldots, 10 \mathrm{~min}$ ), largely decreasing with an increase in the number of Fourier orders being solved for, keeping the number of AUVs, navigation errors, and time steps constant. The errors also decrease as the number of AUVs is increased. With this knowledge and technology we will be able to improve the plume simulation further based on the physics of plume spreading via currents and diffusion, and employ adaptive autonomy behaviors with the AUVs to progress them along the plume boundary. In the end, the plume tracking process presented here will provide a synoptic data set describing the plume based on the spatiotemporal scales of the feature, using a network of AUVs to prevent data aliasing. 


\section{Bibliography}

[1] M. R. Benjamin, H. Schmidt, P. M. Newman, and J. J. Leonard, "Nested autonomy for unmanned marine vehicles with MOOS-IvP," Journal of Field Robotics, vol. 27, no. 6, pp. 834-875, 2010. [Online]. Available: http://dx.doi.org/10.1002/rob.20370

[2] M. R. Benjamin, J. J. Leonard, H. Schmidt, and P. M. Newman, "An overview of MOOS-IvP and a brief users guide to the IvP Helm autonomy software," MIT, Tech. Rep. MIT-CSAIL-TR-2009-028, 2009. [Online]. Available: http://hdl.handle.net/1721.1/45569

[3] S. Petillo, A. Balasuriya, and H. Schmidt, "Autonomous adaptive environmental assessment and feature tracking via autonomous underwater vehicles," in OCEANS 2010 IEEE - Sydney, Sydney, Australia, May 2010, pp. 1-9.

[4] S. Petillo, H. Schmidt, and A. Balasuriya, "Constructing a distributed auv network for underwater plume-tracking operations," International Journal of Distributed Sensor Networks: Special Issue on Distributed Mobile Sensor Networks for Hazardous Applications, vol. 2012, 2012, article ID 191235, 12 pages.

[5] S. Petillo and H. Schmidt, "Autonomous and adaptive plume detection and tracking with auvs: Concepts, methods, and available technology," in Proceedings of the 9th IFAC Conference on Manoeuvring and Control of Marine Craft, G. Bruzzone and M. Caccia, Eds., vol. 9, Arenzano, Italy, September 2012, pp. 232-237. [Online]. Available: http://www.ifac-papersonline.net/Detailed/ 64611.html

[6] T. Schneider and H. Schmidt, "The Dynamic Compact Control Language: A compact marshalling scheme for acoustic communications," in Proceedings of the IEEE Oceans Conference 2010, Sydney, Australia, May 2010.

[7] Goby Developers, "Goby underwater autonomy project documentation." [Online]. Available: http://gobysoft.com/doc/1.0

[8] Woods Hole Oceanographic Institution, "WHOI Acoustic Communications: Micro-Modem Overview," accessed 24 August, 2011. [Online]. Available: http://acomms.whoi.edu/umodem/

[9] "SQLite," Accessed 20 November 2014. [Online]. Available: http://www.sqlite.org/

[10] I. The MathWorks, "MATLAB," 2014, Accessed 20 November 2014. [Online]. Available: http://www.mathworks.com/products/matlab/

[11] J. W. Eaton, “GNU Octave," 2013, Accessed 20 November 2014. [Online]. Available: https://www.gnu.org/software/octave/ 
[12] T. C. R. Network, “cplusplus.com),” 2013, Accessed 20 November 2014. [Online]. Available: http://www.cplusplus.com/

[13] M. C. Martin and H. P. Moravec, "Robot evidence grids," The Robotics Institute, Carnegie Mellon University, Pittsburgh, PA, Tech. Rep. CMU-RI-TR-96-06, March 1996.

[14] O. S. R. Foundation, "Ros." [Online]. Available: http://www.ros.org/

[15] A. Huang, E. Olson, and D. Moore, "LCM: Lightweight communications and marshalling," in Proceedings of the IEEE/RSJ International Conference on Intelligent Robots and Systems (IROS), October 2010.

[16] "Ocean instruments: Conductivity, Temperature, Depth (CTD) sensors." [Online]. Available: http://www.whoi.edu/instruments/viewInstrument.do?id=1003

[17] "Sensorex: Dissolved oxygen technical education." [Online]. Available: http://www.sensorex.com/ support/education/DO_education.html

[18] “WET Labs: ECO fluorometer." [Online]. Available: http://www.wetlabs.com/products/eflcombo/ fl.htm

[19] "Satlantic: PAR sensor series." [Online]. Available: http://www.satlantic.com/Radiometers_PAR_ Sensors/Radiometers_PAR_Sensors.asp

[20] "Ocean instruments: Acoustic Doppler Current Profiler (ADCP)." [Online]. Available: http: //www.whoi.edu/instruments/viewInstrument.do?id=819

[21] T. W. R. T. T. Incorporated, “Slocum Glider," 2010, accessed 1 October, 2014. [Online]. Available: http://www.webbresearch.com/slocumglider.aspx

[22] I. D. G. at Scripps Institution of Oceanography, "Underwater Gliders," accessed 1 October, 2014. [Online]. Available: http://spray.ucsd.edu/pub/rel/info/spray_description.php

[23] E. D. LLC, "Exocetus Coastal Glider," 2012, accessed 1 October, 2014. [Online]. Available: http://exocetus.com/glider/

[24] S. F. C. S. of the University of Washington, “Seaglider Fabrication Center," accessed 1 October, 2014. [Online]. Available: http://seaglider.washington.edu/

[25] A. P. L. U. of Washington, “Seaglider," accessed 1 October, 2014. [Online]. Available: http://www.apl.washington.edu/projects/seaglider/summary.html

[26] K. M. AS, "Autonomous Underwater Vehicle - Seaglider," 2014, accessed 1 October, 2014. [Online]. Available: http://www.km.kongsberg.com/ks/web/nokbg0240.nsf/AllWeb/ EC2FF8B58CA491A4C1257B870048C78C?OpenDocument

[27] iRobot, “1KA Seaglider,” accessed 1 October, 2014. [Online]. Available: http://m.irobot.com/ maritime_product.php?product=seaglider

[28] B. R. Corporation, "Bluefin robotics: A battelle company," 2014, accessed 1 October, 2014. [Online]. Available: http://www.bluefinrobotics.com/ 
[29] A. U. V. A. Center, "AUV System Spec Sheet: CMRE Ocean Explorer configuration," accessed 1 October, 2014. [Online]. Available: http://auvac.org/configurations/view/234

[30] O. S. L. at Woods Hole Oceanographic Institution, "REMUS," 2014, accessed 1 October, 2014. [Online]. Available: http://www.whoi.edu/main/remus

[31] H. K. M. AS, "Hydroid: Autonomous Underwater Vehicles," 2014, accessed 1 October, 2014. [Online]. Available: http://www.km.kongsberg.com/hydroid

[32] I. OceanServer Technology, “OceanServer IVER2 Autonomous Underwater Vehicle," 2014, accessed 1 October, 2014. [Online]. Available: http://www.iver-auv.com/

[33] G. T. S.r.l., "Folaga AUV," 2011, accessed 1 October, 2014. [Online]. Available: http: //www.graaltech.it/en/project.php?cid=2\&pid=5

[34] N. D. S. F. at Woods Hole Oceanographic Institution, “AUV Sentry," 2014, accessed 1 October, 2014. [Online]. Available: http://www.whoi.edu/main/sentry

[35] W. H. O. Institution, "Autonomous Underwater Vehicle ABE," 2014, accessed 1 October, 2014. [Online]. Available: http://www.whoi.edu/main/ABE

[36] H. S. L. at Woods Hole Oceanographic Institution, "SeaBED-class AUVs," 2014, accessed 1 October, 2014. [Online]. Available: http://www.whoi.edu/page.do?pid=21140

[37] A. L. at MIT Sea Grant, "AUV Lab Vehicles," accessed 1 October, 2014. [Online]. Available: http://auvlab.mit.edu/vehicles/

[38] B. Cushman-Roisin and J.-M. Beckers, Introduction to Geophysical Fluid Dynamics, 2nd ed. Academic Press, 2011.

[39] M. Jakuba, D. Yoerger, A. Bradley, C. German, C. Langmuir, and T. Shank, "Multiscale, multimodal AUV surveys for hydrothermal vent localization," in Proceedings of the Fourteenth International Symposium on Unmanned Untethered Submersible Technology (UUST05), Durham, NH, 2005.

[40] D. Wang, P. F. Lermusiaux, P. J. Haley, D. Eickstedt, W. G. Leslie, and H. Schmidt, "Acoustically focused adaptive sampling and on-board routing for marine rapid environmental assessment," Journal of Marine Systems, vol. 78, no. Supplement 1, pp. S393 - S407, 2009. [Online]. Available: http://www.sciencedirect.com/science/article/pii/S0924796309001675

[41] R. N. Smith, Y. Chao, P. P. Li, D. A. Caron, B. H. Jones, and G. S. Sukhatme, "Planning and implementing trajectories for autonomous underwater vehicles to track evolving ocean processes based on predictions from a regional ocean model," International Journal of Robotics Research, vol. 29, pp. 1475-1497, October 2010. [Online]. Available: http://dx.doi.org/10.1177/0278364910377243

[42] J. Das, K. Rajan, S. Frolov, F. Py, J. Ryan, D. Caron, and G. Sukhatme, "Towards marine bloom trajectory prediction for AUV mission planning," in Proceedings of the IEEE International Conference on Robotics and Automation (ICRA) 2010, May 2010, pp. $4784-4790$.

[43] R. He, K. Chen, T. Moore, and M. Li, "Mesoscale variations of sea surface temperature and ocean color patterns at the mid-atlantic bight shelfbreak," Geophysical Research Letters, vol. 37, no. 9, pp. n/a-n/a, 2010. [Online]. Available: http://dx.doi.org/10.1029/2010GL042658 
[44] R. He, K. Chen, K. Fennel, G. G. Gawarkiewicz, and D. J. McGillicuddy Jr, "Seasonal and interannual variability of physical and biological dynamics at the shelfbreak front of the middle atlantic bight: nutrient supply mechanisms," Biogeosciences, vol. 8, no. 10, pp. 2935-2946, 2011. [Online]. Available: http://www.biogeosciences.net/8/2935/2011/

[45] K. Carder, D. Costello, H. Warrior, L. Langebrake, W. Hou, J. Patten, and E. Kaltenbacher, "Oceanscience mission needs: real-time auv data for command, control, and model inputs [west florida shelf]," Oceanic Engineering, IEEE Journal of, vol. 26, no. 4, pp. 742-751, Oct 2001.

[46] R. Camilli, C. M. Reddy, D. R. Yoerger, B. A. S. Van Mooy, M. V. Jakuba, J. C. Kinsey, C. P. McIntyre, S. P. Sylva, and J. V. Maloney, "Tracking hydrocarbon plume transport and biodegradation at Deepwater Horizon,” Science, vol. 330, no. 6001, pp. 201-204, 2010. [Online]. Available: http://www.sciencemag.org/content/330/6001/201.abstract

[47] Y. Zhang, A. Baggeroer, and J. Bellingham, "Spectral-feature classification of oceanographic processes using an autonomous underwater vehicle," Oceanic Engineering, IEEE Journal of, vol. 26, no. 4, pp. 726-741, Oct 2001.

[48] N. Yilmaz, C. Evangelinos, P. F. J. Lermusiaux, and N. Patrikalakis, "Path planning of autonomous underwater vehicles for adaptive sampling using mixed integer linear programming," Oceanic Engineering, IEEE Journal of, vol. 33, no. 4, pp. 522-537, Oct 2008.

[49] F. Hover, "Path planning for data assimilation in mobile environmental monitoring systems," in Intelligent Robots and Systems, 2009. IROS 2009. IEEE/RSJ International Conference on, Oct 2009, pp. 213-218.

[50] J. Das, F. Py, T. Maughan, T. O’Reilly, M. Messie, J. Ryan, K. Rajan, and G. Sukhatme, "Simultaneous tracking and sampling of dynamic oceanographic features with autonomous underwater vehicles and Lagrangian drifters," in Proceedings of the 12th International Symposium on Experimental Robotics, New Delhi, India, December 2010.

[51] H. Schmidt, M. R. Benjamin, S. Petillo, T. Schneider, and R. Lum, Springer Handbook of Ocean Engineering. Springer, Berlin, Heidelberg, 2015, ch. Nested Autonomy for Distributed Ocean Sensing, in press.

[52] N. Cruz and A. Matos, "Reactive auv motion for thermocline tracking," in OCEANS 2010 IEEE Sydney, May 2010, pp. 1-6.

[53] _ _Adaptive sampling of thermoclines with autonomous underwater vehicles," in OCEANS 2010, Sept 2010, pp. 1-6.

[54] Y. Zhang, J. Bellingham, M. Godin, J. Ryan, R. McEwen, B. Kieft, B. Hobson, and T. Hoover, "Thermocline tracking based on peak-gradient detection by an autonomous underwater vehicle," in OCEANS 2010, Sept 2010, pp. 1-4.

[55] Y. Zhang, R. McEwen, J. Ryan, and J. Bellingham, "Design and tests of an adaptive triggering method for capturing peak samples in a thin phytoplankton layer by an autonomous underwater vehicle," Oceanic Engineering, IEEE Journal of, vol. 35, no. 4, pp. 785-796, Oct 2010.

[56] Y. Zhang, J. Bellingham, M. Godin, and J. Ryan, "Using an autonomous underwater vehicle to track the thermocline based on peak-gradient detection," Oceanic Engineering, IEEE Journal of, vol. 37, no. 3, pp. 544-553, July 2012. 
[57] F. Cazenave, Y. Zhang, E. McPhee-Shaw, J. G. Bellingham, and T. P. Stanton, "High-resolution surveys of internal tidal waves in Monterey Bay, California, using an autonomous underwater vehicle."

[58] “Windows to the universe." [Online]. Available: http://www.windows.ucar.edu/tour/link=/earth/ Water/images/temperature_depth_jpg_image.html

[59] “Merriam-Webster online.” [Online]. Available: http://www.merriam-webster.com/dictionary/ thermocline

[60] "Multidisciplinary Simulation, Estimation, and Assimilation Systems (MSEAS)." [Online]. Available: http://mseas.mit.edu/

[61] Unidata, "Network Common Data Form (NetCDF)," Accessed 20 November 2014. [Online]. Available: http://www.unidata.ucar.edu/software/netcdf/

[62] "MSEAS re-analyses for the AWACS-SW06 exercise in the Middle Atlantic Bight region." [Online]. Available: http://mseas.mit.edu/Research/AWACS/Model_Output/

[63] T. Schneider, "Google Earth interface for Ocean Vehicles (GEOV)." [Online]. Available: http://aubergine.whoi.edu/geov/index.php

[64] “Google Earth.” [Online]. Available: http://earth.google.com/ocean/

[65] K. V. MacKenzie, "Nine-term equation for the sound speed in the oceans," The Journal of the Acoustical Society of America, vol. 70, no. 3, pp. 807-812, September 1981.

[66] S. Petillo and H. Schmidt, "Exploiting adaptive and collaborative auv autonomy for detection and characterization of internal waves," Oceanic Engineering, IEEE Journal of, vol. 39, no. 1, pp. 150-164, Jan 2014.

[67] N. P. Fofonoff and R. C. Millard, "Algorithms for computation of fundamental properties of seawater," Unesco technical papers in marine science, vol. 44, 1983. [Online]. Available: http://unesdoc.unesco.org/images/0005/000598/059832eb.pdf

[68] J. S. Turner, Buoyancy Effects in Fluids, ser. Cambridge Monographs on Mechanics and Applied Mathematics. Cambridge University Press, 1973.

[69] P. Kundu and I. Cohen, Fluid Mechanics. Academic Press, 2008.

[70] J. Rodenas and R. Garello, "Internal wave detection and location in sar images using wavelet transform," Geoscience and Remote Sensing, IEEE Transactions on, vol. 36, no. 5, pp. 1494 -1507, sep 1998.

[71] R. F. Gasparovic, R. K. Raney, and R. C. Beal, "Ocean remote sensing research and applications at apl," Johns Hopkins APL, Tech. Rep. 4, 1999. [Online]. Available: http: //www.jhuapl.edu/techdigest/TD/td2004/gaspar.pdf

[72] P. Brandt, A. Rubino, W. Alpers, and J. O. Backhaus, "Internal waves in the strait of messina studied by a numerical model and synthetic aperture radar images from the ers $1 / 2$ satellites," Journal of Physical Oceanography, vol. 27, no. 5, pp. 648-663, 1997.

[73] B. D. Dushaw, "A review of internal tide observations by acoustic tomography and altimetry," in Proceedings of the 5th Pacific Ocean Remote Sensing Conference (PORSEC), vol. 2, Goa, India, December 2000, pp. 651-652. [Online]. Available: http://staff.washington.edu/dushaw/ 
[74] A. R. Osborne and T. L. Burch, "Internal solitons in the andaman sea," Science, vol. 208, no. 4443, pp. pp. 451-460, 1980. [Online]. Available: http://www.jstor.org/stable/1683264

[75] P. Baines, "Satellite observations of internal waves on the australian north-west shelf," Marine and Freshwater Research, vol. 32, no. 3, pp. 457-463, 01 1981. [Online]. Available: http: //www.publish.csiro.au/paper/MF9810457

[76] Y. Zhang, A. Baggeroer, and J. Bellingham, "Spectral-feature classification of oceanographic processes using an autonomous underwater vehicle," Oceanic Engineering, IEEE Journal of, vol. 26, no. 4, pp. $726-741$, October 2001.

[77] F. Cazenave, "Internal waves over the continental shelf in south monterey bay," Master's thesis, San Jose State University, 2008, paper 3506. [Online]. Available: http://scholarworks.sjsu.edu/etd_theses/3506

[78] M. Astraldi and G. P. Gasparini, Seasonal and Interannual Variability of the Western Mediterranean Sea, ser. Coastal and Estuarine Studies. Washington, D. C.: AGU, 1994, vol. 46, ch. 7: The Seasonal Characteristics of the Circulation in the Tyrrhenian Sea.

[79] S. Marullo, R. Santoleri, and F. Bignami, Seasonal and Interannual Variability of the Western Mediterranean Sea, ser. Coastal and Estuarine Studies. Washington, D. C.: AGU, 1994, vol. 46, ch. 8: The Surface Characteristics of the Tyrrhenian Sea: Historical Satellite Data Analysis.

[80] R. Santoleri, E. Böhm, and M. E. Schiano, Seasonal and Interannual Variability of the Western Mediterranean Sea, ser. Coastal and Estuarine Studies. Washington, D. C.: AGU, 1994, vol. 46, ch. 9: The Sea Surface Temperature of the Western Mediterranean Sea: Historical Satellite Thermal Data.

[81] S. Kemna, M. Hamilton, D. Hughes, and K. LePage, "Adaptive autonomous underwater vehicles for littoral surveillance," Intelligent Service Robotics, vol. 4, pp. 245-258, 2011, 10.1007/s11370-0110097-4. [Online]. Available: http://dx.doi.org/10.1007/s11370-011-0097-4

[82] "MicroCAT C-T (P optional) Sensor SBE 37-SI." [Online]. Available: http://www.seabird.com/ products/spec_sheets/37sidata.htm

[83] Paroscientific, Inc., "Depth Sensors," 2014, accessed 1 October, 2014. [Online]. Available: http://www.paroscientific.com/depthsensors.htm

[84] J. Hopkins, P. Challenor, and A. G. P. Shaw, "A new statistical modeling approach to ocean front detection from SST satellite images," J. Atmos. Oceanic Technol., 2010.

[85] R. Legeckis, "A survey of worldwide sea surface temperature fronts detected by environmental satellites," Journal of Geophysical Research: Oceans, vol. 83, no. C9, pp. 4501-4522, 1978. [Online]. Available: http://dx.doi.org/10.1029/JC083iC09p04501

[86] S. M. Mesick, M. H. Booda, and B. Gibson, "Automated detection of oceanic fronts and eddies from remotely sensed satellite data using ARC/INFO," in ESRI International User Conference, Planning Systems Inc., Stennis Center, Stennis Space Center, MS. San Diego, CA: ESRI, 1998.

[87] M. Rixen, J. T. Allen, S. Alderson, V. Cornell, N. Crisp, S. Fielding, A. T. Mustard, R. T. Pollard, E. E. Popova, D. A. Smeed, M. A. Srokosz, A. Barth, and J.-M. Beckers, "Along or across front survey strategy? an operational example at an unstable front," Geophysical Research Letters, vol. 30, no. 1, pp. 17-1-17-4, 2003. [Online]. Available: http://dx.doi.org/10.1029/2002GL015341 
[88] M. Rixen, J. T. Allen, R. T. Pollard, and J.-M. Beckers, "Along or across front ocean survey strategy? the estimation of quasi-geostrophic vertical velocities and temperature fluxes," Geophysical Research Letters, vol. 30, no. 5, pp. n/a-n/a, 2003. [Online]. Available: http://dx.doi.org/10.1029/2002GL015810

[89] E. R. Levine and W. B. White, "Thermal frontal zones in the eastern mediterranean sea," Journal of Geophysical Research, vol. 77, no. 6, pp. 1081-1086, 1972. [Online]. Available: http://dx.doi.org/10.1029/JC077i006p01081

[90] J. Gottlieb, R. Graham, T. Maughan, F. Py, G. Elkaim, and K. Rajan, "An experimental momentumbased front detection method for autonomous underwater vehicles," in Robotics and Automation (ICRA), 2012 IEEE International Conference on, May 2012, pp. 5322-5327.

[91] B. Reed and F. Hover, "Tracking ocean fronts with multiple vehicles and mixed communication losses," in Intelligent Robots and Systems (IROS), 2013 IEEE/RSJ International Conference on, Nov 2013, pp. $3374-3381$.

[92] C. Cannell and D. Stilwell, "A comparison of two approaches for adaptive sampling of environmental processes using autonomous underwater vehicles," in OCEANS, 2005. Proceedings of MTS/IEEE, Sept 2005, pp. 1514-1521 Vol. 2.

[93] Y. Zhang, M. Godin, J. Bellingham, and J. Ryan, "Using an autonomous underwater vehicle to track a coastal upwelling front," Oceanic Engineering, IEEE Journal of, vol. 37, no. 3, pp. 338-347, July 2012.

[94] — "Ocean front detection and tracking by an autonomous underwater vehicle," in OCEANS 2011, Sept 2011, pp. 1-4.

[95] H. Schmidt, M. Benjamin, A. Balasuriya, D. Battle, T. Schneider, and K. Cockrell, "MOOS-IvP nested autonomy prototype for distributed undersea sensing user's guide," MIT, Tech. Rep., 2009. [Online]. Available: http://acoustics.mit.edu/faculty/henrik/LAMSS/lams_nested.pdf

[96] W. G. Leslie, P. J. Haley, Jr., P. F. J. Lermusiaux, M. P. Ueckermann, O. Logutov, and J. Xu, "MSEAS Manual," Department of Mechanical Engineering, Massachusetts Institute of Technology, Cambridge, MA, MSEAS Report 06, 2010. [Online]. Available: http://mseas.mit.edu/?p=2237

[97] P. J. Haley, Jr. and P. F. J. Lermusiaux, "Multiscale two-way embedding schemes for free-surface primitive equations in the "Multidisciplinary Simulation, Estimation and Assimilation System"," Ocean Dynamics, vol. 60, no. 6, pp. 1497-1537, Dec. 2010.

[98] M. P. Ueckermann and P. F. J. Lermusiaux, "High order schemes for 2D unsteady biogeochemical ocean models," Ocean Dynamics, vol. 60, no. 6, pp. 1415-1445, Dec. 2010.

[99] M. P. Ueckermann, P. F. J. Lermusiaux, P. J. Haley, Jr., and C. Mirabito, "Hybridizable discontinuous Galerkin projection methods for Navier-Stokes," Journal of Computational Physics, 2014, to be submitted.

[100] — - "High order schemes for 3D unsteady biogeochemical ocean models," Ocean Dynamics, 2014, to be submitted.

[101] P. J. Haley, Jr., A. Agarwal, and P. F. J. Lermusiaux, "Optimizing velocities and transports for complex coastal regions and archipelagos," Ocean Modeling, 2014, sub-judice. 
[102] O. G. Logutov and P. F. J. Lermusiaux, "Inverse barotropic tidal estimation for regional ocean applications," Ocean Modelling, vol. 25, no. 1-2, pp. 17-34, 2008. [Online]. Available: http://www.sciencedirect.com/science/article/pii/S1463500308000851

[103] A. Agarwal and P. F. J. Lermusiaux, "Statistical field estimation for complex coastal regions and archipelagos," Ocean Modelling, vol. 40, no. 2, pp. 164-189, 2011.

[104] P. F. J. Lermusiaux, "Uncertainty estimation and prediction for interdisciplinary ocean dynamics," Journal of Computational Physics, vol. 217, pp. 176-199, 2006.

[105] A. Phadnis, "Uncertainty quantification and prediction for non-autonomous linear and nonlinear systems," Master's thesis, Massachusetts Institute of Technology, Department of Mechanical Engineering, Cambridge, Massachusetts, September 2013.

[106] T. Sondergaard and P. F. J. Lermusiaux, "Data assimilation with Gaussian Mixture Models using the Dynamically Orthogonal field equations. Part I: Theory and scheme." Monthly Weather Review, vol. 141, no. 6, pp. 1737-1760, 2013.

[107] _ _ "Data assimilation with Gaussian Mixture Models using the Dynamically Orthogonal field equations. Part II: Applications.” Monthly Weather Review, vol. 141, no. 6, pp. 1761-1785, 2013.

[108] P. F. J. Lermusiaux, “Adaptive modeling, adaptive data assimilation and adaptive sampling," Physica D: Nonlinear Phenomena, vol. 230, no. 1, pp. 172-196, 2007.

[109] T. P. Sapsis and P. F. J. Lermusiaux, "Dynamically orthogonal field equations for continuous stochastic dynamical systems," Physica D: Nonlinear Phenomena, vol. 238, no. 23-24, pp. 2347-2360, Dec. 2009, doi:10.1016/j.physd.2009.09.017.

[110] _ - "Dynamical criteria for the evolution of the stochastic dimensionality in flows with uncertainty," Physica D: Nonlinear Phenomena, vol. 241, no. 1, pp. 60-76, 2012.

[111] M. P. Ueckermann, P. F. J. Lermusiaux, and T. P. Sapsis, "Numerical schemes for dynamically orthogonal equations of stochastic fluid and ocean flows," Journal of Computational Physics, vol. 233, pp. 272-294, Jan. 2013.

[112] P. G. Y. Lu, "Bayesian inference of stochastic dynamical models," Master's thesis, Massachusetts Institute of Technology, Department of Mechanical Engineering, Cambridge, Massachusetts, February 2013.

[113] W. G. Leslie, A. R. Robinson, P. J. Haley, Jr, O. Logutov, P. A. Moreno, P. F. J. Lermusiaux, and E. Coelho, "Verification and training of real-time forecasting of multi-scale ocean dynamics for maritime rapid environmental assessment," Journal of Marine Systems, vol. 69, no. 1, pp. 3-16, 2008.

[114] R. Onken, A. Álvarez, V. Fernández, G. Vizoso, G. Basterretxea, J. Tintoré, P. Haley, Jr., and E. Nacini, "A forecast experiment in the Balearic Sea." Journal of Marine Systems, vol. 71, no. 1-2, pp. 79-98, 2008.

[115] P. J. Haley, Jr., P. F. J. Lermusiaux, A. R. Robinson, W. G. Leslie, O. Logoutov, G. Cossarini, X. S. Liang, P. Moreno, S. R. Ramp, J. D. Doyle, J. Bellingham, F. Chavez, and S. Johnston, "Forecasting and reanalysis in the Monterey Bay/California Current region for the Autonomous Ocean Sampling Network-II experiment," Deep Sea Research Part II: Topical Studies in Oceanography, vol. 56, no. 3-5, pp. 127-148, Feb. 2009, doi:10.1016/j.dsr2.2008.08.010. 
[116] P. F. J. Lermusiaux, P. J. Haley, W. G. Leslie, A. Agarwal, O. Logutov, and L. J. Burton, "Multiscale physical and biological dynamics in the Philippine Archipelago: Predictions and processes," Oceanography, vol. 24, no. 1, pp. 70-89, 2011, Special Issue on the Philippine Straits Dynamics Experiment.

[117] A. Gangopadhyay, P. F. Lermusiaux, L. Rosenfeld, A. R. Robinson, L. Calado, H. S. Kim, W. G. Leslie, and P. J. Haley, Jr., "The California Current system: A multiscale overview and the development of a feature-oriented regional modeling system (FORMS)," Dynamics of Atmospheres and Oceans, vol. 52, no. 1-2, pp. 131-169, Sep. 2011, Special issue of Dynamics of Atmospheres and Oceans in honor of Prof. A. R. Robinson.

[118] S. R. Ramp, P. F. J. Lermusiaux, I. Shulman, Y. Chao, R. E. Wolf, and F. L. Bahr, "Oceanographic and atmospheric conditions on the continental shelf north of the Monterey Bay during August 2006," Dynamics of Atmospheres and Oceans, vol. 52, no. 1-2, pp. 192-223, Sep. 2011, Special issue of Dynamics of Atmospheres and Oceans in honor of Prof. A. R. Robinson.

[119] M. E. G. D. Colin, T. F. Duda, L. A. te Raa, T. van Zon, P. J. Haley, Jr., P. F. J. Lermusiaux, W. G. Leslie, C. Mirabito, F. P. A. Lam, A. E. Newhall, Y.-T. Lin, and J. F. Lynch, "Time-evolving acoustic propagation modeling in a complex ocean environment," in OCEANS - Bergen, 2013 MTS/IEEE, 2013, pp. 1-9.

[120] P. F. J. Lermusiaux, P. J. Haley, Jr., G. Gawarkiewicz, and S. Jan, "Evaluation of ocean probabilistic forecasts: Quantifying, predicting and exploiting uncertainty," Ocean Dynamics, 2014, to be submitted.

[121] P. F. J. Lermusiaux, P. J. Haley, Jr, and N. K. Yilmaz, "Environmental prediction, path planning and adaptive sampling: sensing and modeling for efficient ocean monitoring, management and pollution control," Sea Technology, vol. 48, no. 9, pp. 35-38, 2007.

[122] J. Xu, P. F. J. Lermusiaux, P. J. Haley Jr., W. G. Leslie, and O. G. Logutov, "Spatial and Temporal Variations in Acoustic propagation during the PLUSNet-07 Exercise in Dabob Bay," in Proceedings of Meetings on Acoustics (POMA), vol. 4. Acoustical Society of America 155th Meeting, 2008, p. 11.

[123] G. Cossarini, P. F. J. Lermusiaux, and C. Solidoro, "Lagoon of Venice ecosystem: Seasonal dynamics and environmental guidance with uncertainty analyses and error subspace data assimilation," Journal of Geophysical Research: Oceans, vol. 114, no. C6, Jun. 2009, doi:10.1029/2008JC005080.

[124] "MSEAS re-analyses for the AWACS-SW06 exercise in the Middle Atlantic Bight region." [Online]. Available: http://mseas.mit.edu/Research/SW06/MSEAS_reanalysis/2013_May07/MSEAS_Output/

[125] WHOI, "Shallow water experiment 2006," 2006, Accessed 31 October 2014. [Online]. Available: http://acoustics.whoi.edu/sw06/

[126] P. F. J. Lermusiaux, P. J. Haley, Jr., W. G. Leslie, O. Logoutov, and A. R. Robinson, "Autonomous Wide Aperture Cluster for Surveillance (AWACS): Adaptive Sampling and Search Using Predictive Models with Coupled Data Assimilation and Feedback - Harvard Page," MSEAS Sea Exercises, 2006, Accessed 31 October 2014. [Online]. Available: http://mseas.mit.edu/archive/AWACS/index_AWACS.html

[127] N. R. Chapman and J. F. Lynch, “Special issue on the 2006 shallow water experiment," IEEE Journal of Oceanic Engineering, vol. 35, no. 1, pp. 1-2, 2010. 
[128] Y.-T. Lin, A. E. Newhall, T. F. Duda, P. F. J. Lermusiaux, and P. J. Haley, "Merging multiple-partialdepth data time series using objective empirical orthogonal function fitting," IEEE Journal of Oceanic Engineering, vol. 35, no. 4, pp. 710-721, Oct. 2010.

[129] NOAA, "Integrated models of coastal relief," National Geophysical Data Center, 2006, Accessed 31 October 2014. [Online]. Available: http://www.ngdc.noaa.gov/mgg/coastal/coastal.html

[130] W. H. F. Smith and D. T. Sandwell, "Global seafloor topography from satellite altimetry and ship depth soundings." Science, vol. 277, no. 5334, pp. 1957-1962, 1997.

[131] T. P. Boyer, J. I. Antonov, O. K. Baranova, C. Coleman, H. E. Garcia, A. Grodsky, D. R. Johnson, R. A. Locarnini, A. V. Mishonov, T. D. O’Brien, C. R. Paver, J. R. Reagan, D. Seidov, I. V. Smolyar, and M. M. Zweng, "World ocean database 2013," US Government Printing Office, Washington, DC., NOAA Atlas NESDIS 72, 2013, S. Levitus and Alexey Mishonov (ed.s).

[132] C. Sun, A. Thresher, R. Keeley, N. Hall, M. Hamilton, P. Chinn, A. Tran, G. Goni, L. P. de la Villeon, T. Carval, L. Cowen, G. Manzella, V. Gopalakrishna, R. Guerrero, F. Reseghetti, Y. Kanno, B. Klein, L. Rickards, A. Baldoni, S. Lin, F. Ji, and Y. Nagaya, "The data management system for the Global Temperature and Salinity Profile Programme," in Proceedings of OceanObs.09: Sustained Ocean Observations and Information for Society, J. Hall, D. E. Harrison, and D. Stammer, Eds., vol. 2. ESA Publication, 2010.

[133] R. A. Locarnini, A. V. Mishonov, J. I. Antonov, T. P. Boyer, and H. E. Garcia, "World Ocean Atlas 2005, Volume 1: Temperature," US Government Printing Office, Washington, DC., NOAA Atlas NESDIS 61, 2006, S. Levitus (ed.).

[134] J. I. Antonov, R. A. Locarnini, T. P. Boyer, A. V. Mishonov, and H. E. Garcia, "World Ocean Atlas 2005, Volume 2: Salinity," US Government Printing Office, Washington, DC., NOAA Atlas NESDIS 62, 2006, S. Levitus (ed.).

[135] N. Q. Sloan, III, "Dynamics of a shelf-slope front: Process studies and data-driven simulations in the middle atlantic bight." Ph.D. dissertation, Harvard University, Cambridge, Massachusetts, 1996.

[136] P. F. J. Lermusiaux, "Data assimilation via Error Subspace Statistical Estimation, part II: Mid-Atlantic Bight shelfbreak front simulations, and ESSE validation," Monthly Weather Review, vol. 127, no. 7, pp. 1408-1432, Jul. 1999.

[137] A. Gangopadhyay, A. R. Robinson, P. J. Haley, W. G. Leslie, C. J. Lozano, J. J. Bisagni, and Z. Yu, "Feature-oriented regional modeling and simulations in the Gulf of Maine and Georges Bank," Continental Shelf Research, vol. 23, no. 3-4, pp. 317-353, 2003.

[138] W. C. Skamarock, J. B. Klemp, J. Dudhia, D. O. Gill, D. M. Barker, M. Duda, X. Y. Huang, W. Wang, and J. G. Powers, "A description of the advanced research wrf version 3," NCAR, Boulder, CO., NCAR technical note, 2008.

[139] T. E. Rosmond, J. Teixeira, M. Peng, T. F. Hogan, and R. Pauley, "Navy operational global atmospheric prediction system (nogaps): Forcing for ocean models," Oceanography, vol. 15, no. 1, pp. 99-108, 2002.

[140] G. D. Egbert and S. Y. Erofeeva, "Efficient inverse modeling of barotropic ocean tides." Journal of Atmospheric and Oceanic Technology, vol. 19, no. 2, pp. 183-204, 2002. 
[141] J. Farrell, S. Pang, and W. Li, "Chemical plume tracing via an autonomous underwater vehicle," IEEE Journal of Oceanic Engineering, vol. 30, no. 2, pp. 428- 442, April 2005.

[142] S. Pang and J. Farrell, "Chemical plume source localization," IEEE Transactions on Systems, Man, and Cybernetics, Part B: Cybernetics, vol. 36, no. 5, pp. 1068-1080, October 2006.

[143] S. Pang, "Plume source localization for auv based autonomous hydrothermal vent discovery," in Proceedings of the IEEE Oceans Conference 2010, September 2010, pp. 1-8.

[144] C. Cannell, A. Gadre, and D. Stilwell, "Boundary tracking and rapid mapping of a thermal plume using an autonomous vehicle," in Proceedings of the IEEE Oceans Conference 2006, September 2006, pp. 1-6.

[145] National Oceanic and Atmospheric Administration's Pacific Marine Environmental Laboratory, "VENTS program: Vent fluid chemistry," accessed 21 March, 2012. [Online]. Available: http://www.pmel.noaa.gov/vents/chemistry/ffluid.html

[146] E. Baker, C. German, and H. Elderfield, Seafloor Hydrothermal Systems: Physical, Chemical, Biological, and Geological Interactions. Washington, D.C.: AGU, 1995, ch. Hydrothermal plumes over spreading-center axes: Global distributions and geological inferences, p. 47-71.

[147] National Oceanic and Atmospheric Administration's Pacific Marine Environmental Laboratory, "VENTS program," accessed 21 March, 2012. [Online]. Available: http://www.pmel.noaa.gov/vents/

[148] D. Conrad, "Dive and Discover: Interview with Ko-ichi Nakamura," 2008, accessed 21 Mar., 2012. [Online]. Available: http://www.divediscover.whoi.edu/expedition12/interviews/nakamura.html

[149] Woods Hole Oceanographic Institution, "Oil in the Ocean," 2011, accessed 21 March, 2012. [Online]. Available: http://www.whoi.edu/oil/main

[150] S. Mazumder and K. K. Saha, "Detection of oil seepages in oceans by remote sensing," in Proceedings of the 6th International Conference \& Exposition on Petroleum Geophysics, Kolkata, West Bengal, India, 2006.

[151] P. M. Glibert, Ed., Global Ecology and Oceanography of Harmful Algal Blooms (GEOHAB): HABs in Eutrophic Systems, 2006.

[152] D. R. Yoerger, M. Jakuba, A. M. Bradley, and B. Bingham, “Techniques for deep sea near bottom survey using an autonomous underwater vehicle," The International Journal of Robotics Research, vol. 26, no. 1, pp. 41-54, 2007. [Online]. Available: http://ijr.sagepub.com/content/26/1/41.abstract

[153] J. Willcox, J. Bellingham, Y. Zhang, and A. Baggeroer, "Performance metrics for oceanographic surveys with autonomous underwater vehicles," Oceanic Engineering, IEEE Journal of, vol. 26, no. 4, pp. 711 -725 , October 2001.

[154] “Oceanic Diffusion and Mixing," accessed 17 May, 2011. [Online]. Available: http://flux.ocean. washington.edu/WQ2009/oc515.mixing.pdf

[155] W. H. Press and G. B. Rybicki, "Fast algorithm for spectral analysis of unevenly sampled data," Astrophysical Journal, vol. 338, pp. 277-280, March 1989. [Online]. Available: http: //adsabs.harvard.edu/abs/1989ApJ...338..277P 